\title{
Guilherme ORELLI PAIVA
}

Mémoire présenté en vue de l'obtention du grade de Docteur de Le Mans Université sous le label de L'Université Nantes Angers Le Mans

Thèse en cotutelle avec l'Université de Campinas (UNICAMP), Brésil

École doctorale : SPI

Discipline : 60

Spécialité : Acoustique

Unité de recherche : Laboratoire d'Acoustique de l'Université du Maine (LAUM) — UMR CNRS 6613

Soutenue le 12 décembre 2017

Thèse No: 2017LEMA1045

\section{VIBROACOUSTIC CHARACTERIZATION}

\section{AND SOUND SYNTHESIS OF THE viola caipira}

Caractérisation vibroacoustique et synthèse sonore de la viola caipira

\section{JURY}

Rapporteurs : $\quad$ José Roberto de FRANÇA ARRUDA, Professeur Titulaire, FEM-UNICAMP, Campinas, Brésil Scott COGAN, Chargé de Recherche, FEMTO-ST, Besançon

Examinateurs : $\quad$ Iris BRÉMAUD, Chargée de Recherche, LMGC, Montpellier José ANTUNES, Chercheur Principal, LDA-IST, Lisbonne

Directeur de thèse : $\quad$ François GAUTIER, Professeur des Universités, LAUM, Le Mans

Co-directeur de thèse: José Maria CAMPOS DOS SANTOS, Professeur Titulaire, FEM-UNICAMP, Campinas, Brésil

Co-encadrant de thèse : Frédéric ABLITZER, Maître de Conférence, LAUM, Le Mans

Invité:

Yutaka TOHGI, General manager, YAMAHA Music, Hamamatsu, Japon 



\section{ACKNOWLEDGEMENTS}

I would like to thank first of all my thesis directors, François Gautier, Frédéric Ablitzer and José Maria Campos dos Santos for inspiring me day by day to do science and for everything you have done to make this collaboration Brazil/France happen successfully. I am deeply grateful to François and Frédéric for being so dedicated and helpful, and for receiving me so well in your amazing country. Thank you, José Maria, for your friendship, good mood, for the infinite discussions on scientific (and "extra-scientific") subjects, for having deposited your trust in me since the master's degree. Your contributions to my professional and personal journey are incommensurable.

I would also like to thank Scott Cogan and Jose Roberto Arruda for accepting to report this thesis. I am equally grateful to the other members of the jury whose criticisms will certainly come as an essential contribution for this work.

Thanks to the PAFI team: François, Frédéric, Marthe Curtit, Gautier Michelin, Changwei, Mathieu Sécail, Emmanuel Brasseur. Long life to the PAFI project! To Mathieu Sécail, my special thanks, for the fundamental support in the experimental part of the work and for the fruitful discussions.

My sincere expressions of gratitude to the violists/luthiers/artisans/artists José Esmerindo, Levi Ramiro, Luciano Queiroz and Marcelo Teixeira for receiving me in their workshops and making possible many measurements of different and excellent violas caipiras. Thank you for all the discussions and teachings of a universe sometimes too far from the academy. Thanks to the violists Pedro Gava, Laecio Barros, Jack Lima, Fernando Vesco, Eduardo Povra, Clovis Molessani, Rodolfo Thomazelli, Thiago Rossi, Almir Côrtes and Fabio Lucheti for lending me their instruments for measurements.

Thanks to Rozini Musical Instruments for providing us a beautiful and excellent viola caipira for the experimental studies.

I would like to thank Yamaha Music Japan, specially all my ex co-workers from the Research and Development Department, for making my days in Japan one of the most extraordinary experiences of my life.

My special thanks, from the bottom of my heart, to my dear friend Rodolfo Thomazelli. Thanks for the partnership in the "Viola Expedition" and for opening my curtains to the formidable universe of the viola caipira.

I would also like to thank the professor/researches from LAUM/ENSIM who helped a lot, in particular Joël Gilbert, Pierrick Lotton, Jean-Hugh Thomas, Charles Pezerat and Jean-Michel Génevaux.

Thanks to Julien Poitevin (the "Cameraman"), who helped me a lot with the high-speed camera videos.

All my gratitude to my lab colleagues from DMC (Brazil) and ENSIM (France). Many thanks to Flávio Banwarth for all the essential tips on the charming city of Le Mans. Thanks also to all the students from ENSIM for helping me to obtain numerous experimental results.

I would also like to thank Elisabeth, Anne-Marie Brulé and Valérie Hermann for the availability, readiness and for all the efforts expended on the administrative part of the work. Thanks all those from the CPG/FEM and the École Doctorale of the University of Maine. 
Thanks to my super friends I could meet in France: Martina, Ana, Fred, Celia, Amory, Nelson, Flávia, Haitem, Jamal, Matheus, Katerina, Renan, Marcelo, Ciro, Daniel, Maria, Reinis, Karolis, Gwen, Mehran, Margaux, Vincent Fréour, Yosra, Changwei (the best Chinese ever!). Without your company everything would be much harder. Thanks Ana for the coffees and vinyls. :)

Thanks to Kika, for the complicity without borders.

Finally, I deeply thank all my family: you are my greatest treasure.

This work would not have been possible without the financial support of the Brazilian Coordination for the Improvement of Higher Education Personnel Foundation, National Council for Scientific and Technological Development in Brazil and French National Center for Scientific Research. 
Contents

V 



\section{List of Figures}





\section{List OF TABLES}





\section{Chapter 1}

\section{INTRODUCTION}

\subsection{Context}

Several types of violas are played in numerous cultural manifestations throughout the Brazilian territory. Such chordophones, which we can call in a general way of Brazilian violas, have variant characteristics and are related to musical practices of specific regions. The Brazilian violas differ mainly in the shape of the resonance box, number and arrangement of the strings, composition materials and tuning type. This research is focused on the type of Brazilian viola known as viola caipira, which permeates various genres of traditional and modern Brazilian music, and whose practice extends across all regions of Brazil, being considered one of the Brazilian cultural symbols. Unlike other string instruments such as guitars and violins, the viola caipira is a little explored instrument from the perspective of musical acoustics. This thesis proposes the characterization of this instrument in vibrational and acoustical terms with the objective of identifying its specificities.

The correlation between the structural characteristics of a musical instrument and the subjective evaluation of its sound attributes is an issue investigated for at least five decades, and whose understanding has advanced under contributions from different scientific domains such as the acoustics, mechanics, signal processing, computation and psychology. In this context, two types of methodological approaches complement each other: the objective, which proposes to study the relationship between the instrument and the sound produced through physical parameters; and the perceptive, which analyses the sound produced by the instrument from the sensory experience of the individual - musician and/or listener based on fundamentals and methods of contemporary psychology. In this Ph.D. work, the study of the viola caipira is limited to physical characterization of the instrument involving different methods such as vibration and sound pressure measurements, investigation of string motion using high speed camera, physical modelling for sound synthesis purposes and numerical modelling using the finite element method.

The sound produced by string instruments like the viola capira is the result of the interaction between several subsystems: the mechanisms of excitation associated with the musician (e.g., finger, nail, pick or plectrum), strings, instrument body and finally, the listener in the room where the instrument is played. When the musician applies a gesture on the instrument, force transients are exerted on one or more strings, which in turn resonate freely and transfer to the body of the instrument most of the energy that is converted into radiated sound. Therefore, the interaction between the strings and instrument body, which naturally depends on the physical characteristics of these two subsystems, is an important aspect in the production of sound. In this sense, a set of methods known as physical modelling uses physical equations to describe the vibrational behaviour of the string/body system usually through coupled partial differential equations 
containing information on material properties, geometry and boundary conditions. Given the input parameters describing the distribution of one or more excitation forces and/or initial conditions along the strings, a physical model consists in solving the motion equations numerically by using appropriated approximations methods. The output of the model can be selected at a point, for example, the string displacement at the excitation point or the radiated pressure at a specific point in front of the body. The development of sound synthesis methods based on physical models has grown significantly in the last three decades, mainly due to the advent of technology and consequent improvement in the processing capacity of computers. In this context, this thesis proposes a physical model for sound synthesis able to reproduce the sound specificities of the viola caipira.

Within the scope of musical acoustics, physical models for sound synthesis are also tools of great interest for musical composition and performance, and building of musical instruments. Such models can provide high-quality synthesizers for musicians or even reveal new possibilities of sounds and ways of playing a given instrument. The luthier(French word to designate maker of stringed instruments such as violins and guitars), for example, can use simulations to design or even conceive an instrument according to desired effects so that the building process can be optimized. In this way, it is worth highlighting the importance of complementary dialogue between musical acoustics researchers, musicians and luthiers: despite being guided by different biases, they can exchange demands, questions and understandings. This thesis permeates the context of the musical instruments making. It is a collaboration with the PAFI project, Plateforme d'Aide à la Facture Instrumentale ${ }^{1}$, held in the Acoustics Laboratory of the University of Maine (Le Mans, France), which aims at developing adapted tools for luthiers, whether small or large-scale production.

\subsection{Thesis objectives}

Based on the contextual aspects presented above, the main goals of this thesis are:

- To investigate experimentally the vibrational and acoustical behaviour of the viola caipira in order to identify its specificities. Why does the viola caipira have such a particular timbre? Which phenomena are relevant in the instrument's sound production? These are the main questions to be answered at this stage.

- To present a physical model for sound synthesis able to reproduce the sound specificities of the viola caipira.

\subsection{Thesis organization}

This thesis consists of five chapters and its organization is presented as follows:

- Chapter 1 - In the rest of this chapter the viola caipira is briefly described in organological, historical and socio-cultural terms.

- Chapter 2 - A bibliography review is presented where different aspects on the functioning of plucked string instruments are approached. Previous works on the viola caipira as well as finite difference schemes used in musical sound synthesis are also briefly reviewed.

1. Music Instrument Making Support Platform 
- Chapter 3 - An experimental study of the viola caipira using different methods is presented. First, a high-speed camera is used to analyse a typical pluck of the instrument. Viola caipira sounds are recorded and an analysis is carried out in terms of energy decay curves and spectrograms. Vibrational analyses of the instrument body are performed including a modal analysis of the soundboard and an operating deflection shape (ODS) analysis using an automatic impact hammer and a laser vibrometer. Finally, the mode shape components at the string/body coupling points of a viola caipira are extracted using a high resolution modal analysis of mobility measurements obtained with a novel technique named "Roving Wire-Breaking Technique". This latter part is presented as article entitled "The Roving Wire-Breaking Technique: a low cost mobility measurement procedure for string musical instruments", submitted to the Applied Acoustics Journal in October 2017.

- Chapter 4 - The chapter is structured around the article entitled "Collisions in double string plucked instruments: physical modelling and sound synthesis of the viola caipira", submitted to the Journal of Sound and Vibration in November 2017. In this paper is presented a modal-based model comprising 10 strings with non-planar motions coupled with the body. A finite difference scheme is used to generate a set of viola caipira sounds. The sound characteristics identified experimentally are reproduced and discussed.

- Chapter 5 - The chapter presents the general conclusions of the thesis and perspectives for future works.

\subsection{What is a viola caipira?}

\subsubsection{General description}

Figure ?? shows a typical viola caipira, which is a Brazilian plucked string instrument having, in general, a smaller body with a narrower waist than those of classical guitars ${ }^{2}$. As shown in Figure ??, it usually has ten metal strings ${ }^{3,4}$ arranged in five courses of two strings with the thinnest string (string 6) located in the middle. The first two courses have identical strings tuned in unison while the other three have strings with different diameters tuned in an octave. Strings of the same course are usually played together using the fingernails but a plectrum can be also attached to the thumb. The instrument as we know it today has normally from 17 to 19 frets.

The viola caipira is played in all regions of Brazil but is typical of the South Central region, more specifically the states of São Paulo, southern Minas Gerais, southern Goiás, southeast Mato Grosso do Sul and the Federal District. There is not a single standard of viola caipira since variants of shapes, tuning types, materials and arrangement of strings are commonly encountered in different regions of the country. Although different woods are used in the making of the instrument, the most usual model is Sitka Spruce on the top and Rosewood on the sides and back. Figure ?? shows, for example, four violas caipiras with different shapes of resonance box made with different materials. As variant as the features of the 7).

2. The term "classical guitar" is used in this text to designate the modern classical guitar (see, for example, reference [?], page

3. Originally, the violas caipiras had strings made with animal guts or vegetable fibers.

4. Although not so common, there are violas caipiras with 12 strings arranged in 3 doublets and 2 triplets, 7 strings arranged in 1 triplet and 4 simple strings as well as 5 simple strings so that the strings divided in five courses is a strong characteristic of the instrument.

5. Image retrieved from $<$ http://www.rozini.com.br/default.asp?area $=02 \&$ cat $=5 \&$ Produto $=87>$. 


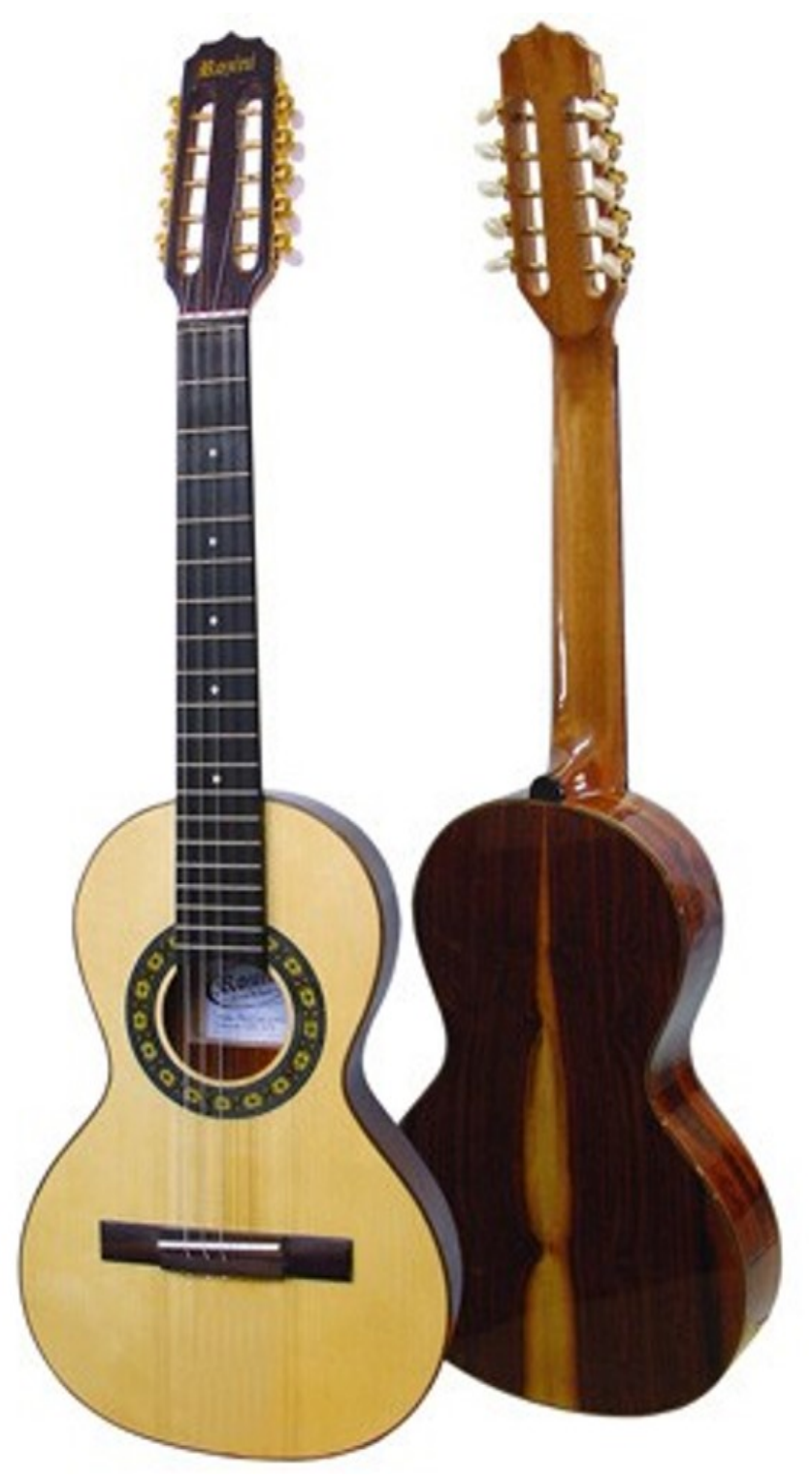

(a) Example of a viola caipira commercialized in large scale in Brazil. ${ }^{5}$

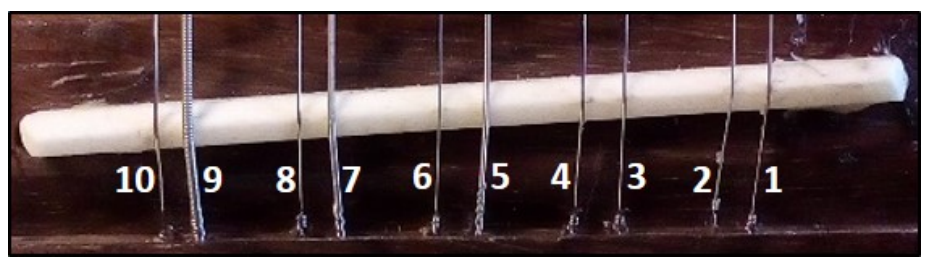

(b) Usual arrangement and numbering of the viola caipira strings.

Figure 1.1: (a) Viola caipira Rozini brand, Ponteio Profissional model; (b) From string 1 to 10, the commom designation is: prima, contra-prima, requinta, contra-requinta, turina, contraturina, toeira, contra-toeira, canotilho and contra-canotilho. The first two courses have identical strings tuned in unison while the other three have strings with different diameters tuned in an octave. Strings 5, 7 and 9 are composed of a metal core covered in wound metal (wound strings) and strings 1, 2, 3, 4, 6, 8 and 10 are composed of a single metal wire (flat strings). 


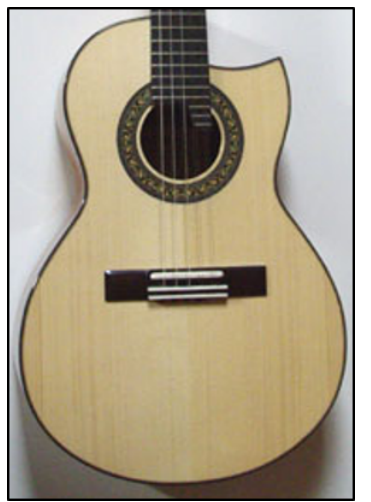

(a) Free High model by Marcelo Teixeira.

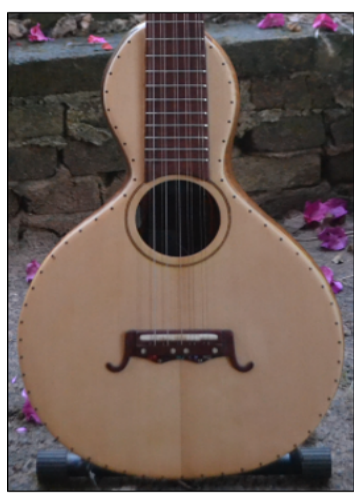

(c) Cabaça model by Levi Ramiro.

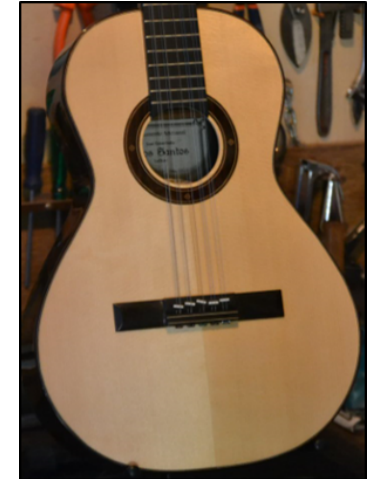

(b) Classic model by José Esmerindo.

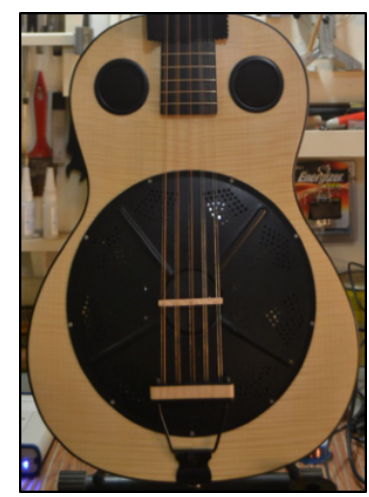

(d) Dinâmica model by Luciano Queiroz.

Figure 1.2: Examples of viola caipira models made with different materials and different resonance box shapes. The respective builders are indicated.

instrument, are the nomenclatures to designate it. Depending on the region and context the instrument is also called viola cabocla, viola sertaneja, viola de pinho, viola de dez cordas, viola de arame, viola cantadeira, viola chorosa, viola de folia, viola nordestina, viola de feira, etc. In this work, the term "viola caipira" is used since it is the most popular.

In spite of the considerable differences between the varieties of violas caipiras, a typical style of the instrument can be identified as the most widespread and played in the Brazilian territory. Figure ?? shows an example of such style. The instrument body shape is similar to those of classical guitars although with a smaller size and slightly narrower waist. The wood types as well as the thicknesses of the soundboard, back plate and sides follow the same patterns used for classical guitars. The traditional fan-bracing style ${ }^{6}$ is also widely used to support and reinforce internally the soundboard and back plate of the instrument. Figure ?? shows the names given to the main components of the viola caipira. Note that they are the same of those of classical guitars.

A little more than a century ago, the structural resemblance between the violas caipira and classical

6. Wright [?] highlights that "many of the standard features of the modern classical guitar (its larger size and fan arrangement of struts) are attributed to Antonio de Torres, although it is true to say that such features were not invented by him. These design features evolved during the early 19th century when the instrument underwent its period of accelerated evolution. Torres started his work at the end of this period, absorbing some of the ideas of earlier luthiers, and through a combination of skilled designs and his association with influential players such as Tarrega, popularised his guitars." 
guitar were not as apparent as today ${ }^{7}$. In the early 20th century, in São Paulo, the viola caipira began to be manufactured in large scale by factories that already produced classical guitars. By applying the same "recipes" and construction techniques that were used for classical guitars, changes were gradually incorporated to the viola caipira throughout the last century so that a pattern of the instrument has somehow consolidated [?]. Excellent detailed accounts of the evolution of the viola caipira and its construction are given by Corrêa [?] and Vilela[?].

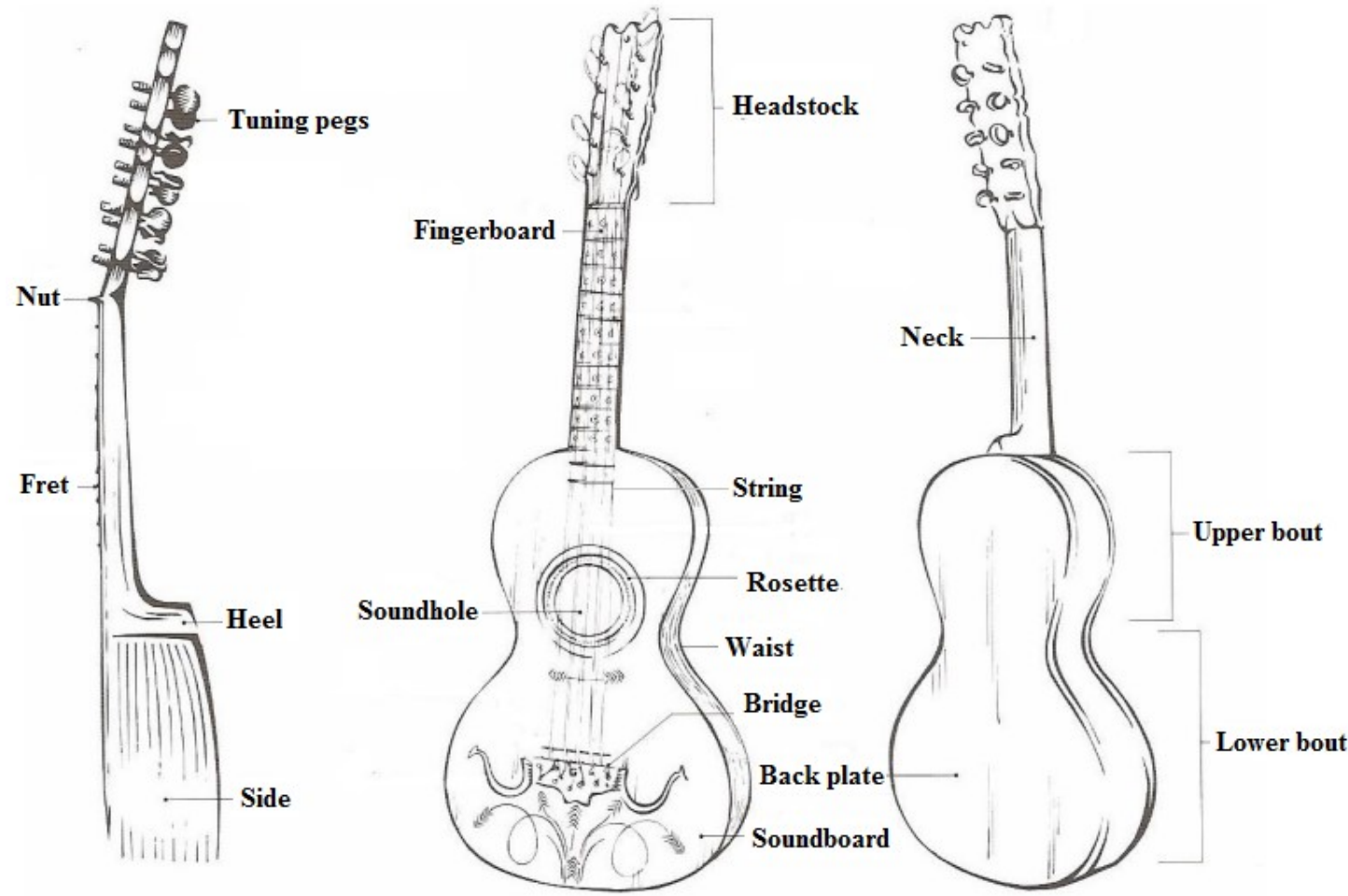

Figure 1.3: Main components of the viola caipira (adapted from [?]).

\subsubsection{Tuning types}

Unlike the classical guitar, which has a consolidated specific tuning ${ }^{8}$, the viola caipira may be played in various tuning types. Vilela [?] points out that from the nine Portuguese tunings that went to Brazil, many others were developed, and that it is estimated that there are approximately twenty ways to tune the viola in Brazil. Some of these tunings are rarely used, while the others are widespread in different regions. While several are used in the same region, there are other regions with a predominance of a certain tuning. Several researchers [?, ?, ?, ?] catalogued many tuning types throughout the Brazilian territory. All these tuning types were compiled by Pedro ([?], page 151).

Although there are numerous tuning types for the viola caipira, the most commonly used ones, especially in the Central South region of Brazil, are the Cebolão D, Cebolão E, Rio Abaixo and Natural, whose notes, respective fundamental frequencies and frequency ranges (lowest and highest notes) are

7. The classical guitar, as we know it today, dates back about 250 years; the ancestor of the viola caipira, in turn, is close to eight hundred years old [?].

8. Classical guitar standard tuning defines the string pitches as E, A, D, G, B, and E, from lowest (low E2) to highest (high E4). 
TABLE 1.1: The most popular viola caipira tuning types and respective notes, fundamental frequencies, lowest notes and highest notes considering an instrument with 19 frets and $A 4=440 \mathrm{~Hz}$.

\begin{tabular}{|c|c|c|c|c|c|c|c|c|}
\hline \multirow[b]{3}{*}{ String No. } & \multicolumn{8}{|c|}{ Tuning types } \\
\hline & \multicolumn{2}{|c|}{ Cebolão E } & \multicolumn{2}{|c|}{ Cebolão D } & \multicolumn{2}{|c|}{ Rio Abaixo } & \multicolumn{2}{|c|}{ Natural } \\
\hline & Note & Freq. $[\mathrm{Hz}]$ & Note & Freq. $[\mathrm{Hz}]$ & Note & Freq. $[\mathrm{Hz}]$ & Note & Freq. $[\mathrm{Hz}]$ \\
\hline 1 & E4 & 329.63 & D4 & 293.67 & D4 & 293.67 & $\mathrm{E} 4$ & 329.63 \\
\hline 2 & E4 & 329.63 & D4 & 293.67 & D4 & 293.67 & E4 & 329.63 \\
\hline 3 & B3 & 246.94 & A3 & 220.00 & B3 & 246.94 & B3 & 246.94 \\
\hline 4 & B3 & 246.94 & A3 & 220.00 & B3 & 246.94 & B3 & 246.94 \\
\hline 5 & G\#3 & 207.65 & F\#3 & 185.00 & G3 & 196.00 & G3 & 196.00 \\
\hline 6 & G\#4 & 415.30 & F\#4 & 369.99 & G4 & 392.00 & G4 & 392.00 \\
\hline 7 & E3 & 164.81 & D3 & 146.80 & D3 & 146.80 & D3 & 146.80 \\
\hline 8 & E4 & 329.63 & D4 & 293.70 & D4 & 293.70 & D4 & 293.70 \\
\hline 9 & B2 & 123.47 & A2 & 110.00 & G2 & 98.00 & A2 & 110.00 \\
\hline 10 & B3 & 246.94 & A3 & 220.00 & G3 & 196.00 & A3 & 220.00 \\
\hline Lowest note & $\mathrm{B} 2$ & 123.47 & $\mathrm{~A} 2$ & 110.00 & $\mathrm{~A} 2$ & 110 & G2 & 98.00 \\
\hline Highest note & D\#6 & 1244.51 & C\#6 & 1108.73 & C\#6 & 1108.73 & D6 & 1174.66 \\
\hline
\end{tabular}

shown in Table ??. The string diameters vary according to the tuning type. In order to establish a reference, Corrêa ([?], page 42) recommends the string diameters for a set of tuning types. More details about string physical properties are given in Chapter 3, Table ??.

It is also important to note from Table ?? that, for a given tuning type, several strings have fundamental frequencies or octaves in common, which makes such strings more susceptible to vibrate sympathetically.

\subsubsection{The origin of the viola caipira}

The purpose of this part is to briefly present some relevant aspects about the origin of the viola caipira, which is obviously related to many other cultural and social aspects of certain regions of Brazil. Since the scope of this thesis lies much more in the musical acoustics perspective, this subsection presents a short synthesis based on some of the numerous works about the instrument in the context of humanities and social sciences $[?, ?, ?, ?, ?, ?, ?, ?]$. For a thorough investigation on the history of the viola caipira, the reader is invited to refer to [?], whose some passages are quoted here.

The Brazilian violas, including the viola caipira, originated from the Portuguese violas. In convergence with such assertion, Pinto [?] lists several researchers who share the same point of view: Sardinha [?], Oliveira [?], Andrade [?], Araújo [?], Cascudo [?], Lima[?], Corrêa [?], Martins [?], Vilela [?]. On the origin of the Brazilian violas, Vilela summarizes:

Viola $^{9}$ (Brazilian five-course guitar) is an instrument that was brought to Brazil by the first people, pioneers and Jesuits. It was used as a tool for the cathequesis. This instrument was transformed correspondently with the new land's development at the hands of bandeirantes (pathfinders), tropeiros (responsible for the transport of goods on donkeys) and popular singers. Along with the configuration of the popular culture in Brazil, viola became the speaker of the people of some regions, such as the south-east. [?]

9. The author uses here the term "viola" to refer to a sparse group of numerous plucked Brazilian violas, including the viola caipira. 
The Portuguese violas, in turn, have their distant origins in the oud, whose origin dates back to the Fertile Crescent ${ }^{10}$ at least 5000 years ago. When the Arabs arrived in the Iberian Peninsula in the year 722, the plucked string instruments existing in the Peninsula were the Celtic harps and the Greek-Roman cithara [?]. The oud ${ }^{11}$ was the first plucked string instrument with a neck in which the tunes could be changed, to reach Europe [?].

Another distant ancestor of the Portuguese violas, but not as distant as the oud, are the Latin guitars, instruments of Arabic-Persian origin that arrived around the thirteenth century in the Iberian peninsula and underwent many transformations and hybridizations until prefigure the violas quinhetistas, which are considered somehow the first prototypes of Portuguese violas [?]. Therefore, the presence of Arabs and their instruments, more specifically ouds and Latin guitars, in the Iberian Peninsula, led to the origin and proliferation of the violas in different regions of Portugal. Vilela highlights that each Portuguese region created its own viola:

Viola braguesa (from Braga) in the North; viola amarantina (from Amarante) or two-hearted viola in the Northeast; viola beiroa (from Beira) in the central region; down below, near Lisbon, viola toeira; and further down the South, in Alentejo, viola campaniça. They varied in size, shape and number of strings, but in most cases had a common feature: five courses of strings. [?]

According to Corrêa [?], there is an important material reference documenting the presence of the five-course viola in Portugal in the sixteenth century. It is an instrument built by Belchior Dias in Lisbon in 1581. This viola is exposed in the Royal College of Music in London and its features are similar to those of the Brazilian violas as we know them today.

With the migration to Brazil, the Portuguese viola kept its shape, but with some variations, while was disseminated through the vast Brazilian territory. However, the violists, also transplanted with the instrument, mixed and germinated, renamed it with several names. In addition, they invented traditions, represented by the creation of different genres and the re-elaboration of other musical elements [?].

\subsubsection{The instrument in the caipira tradition and Brazilian recent music}

It is believed that the caipira music was structured as we know it today between the eighteenth and twentieth centuries, but its roots are founded in more remote times, in the beginning of the colonization of Brazil [?]. The violas ${ }^{13}$ accompany the Brazilian cultural traditions since the Portuguese settlers, bringing their cordophones, arrived in the territory that would become Brazil. These instruments were initially used by the Jesuits in the catechesis of the indigenous peoples and remained for a long time linked to religious practices.

It is safe to say that the viola became the viola caipira as it was consecrated, over the centuries, as a symbol of the music practised by rural people who inhabited several settlements in the South Central region of Brazil from the seventeenth century. The people from these settlements, which we can freely call "the first caipiras", were formed, in general, by miscegenation of Portuguese settlers and Indians. Candido [?] explains that these people became "more caipira" as they lost their Portuguese cultural traits and absorbed traits from aboriginal primitive cultures. Figure ?? reproduces the $O$ Violeiro ${ }^{14}$, a famous

10. The modern-day countries with significant territory within the Fertile Crescent are Iraq, Syria, Lebanon, Cyprus, Jordan, Israel, the State of Palestine, Egypt, as well as the southeastern fringe of Turkey and the western fringes of Iran.

11. Also known as the Arabic lute.

12. Images (a) and (b) were retrieved from <http://collectionsdumusee.philharmoniedeparis.fr/0158896-luth-ud.aspx $>$ and < https : //en.wikipedia.org/wiki/GuitarraLatina $>$, respectively.

13. Here we can consider the numerous types of ancient and modern violas, whether from Portugal or Brazil, including the viola caipira.

14. "The Violist" in english. 


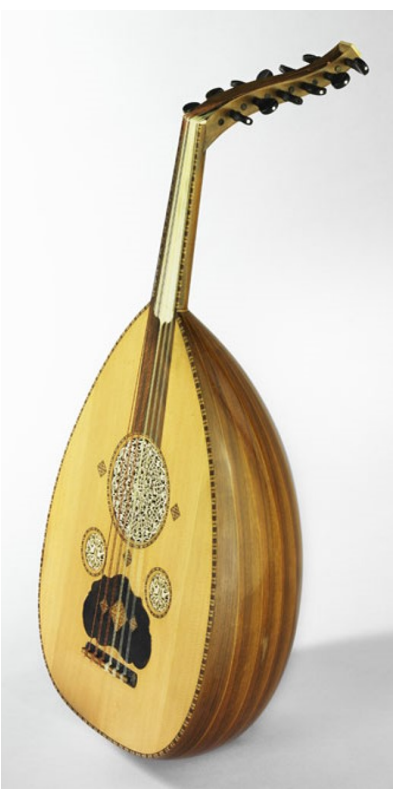

(a)

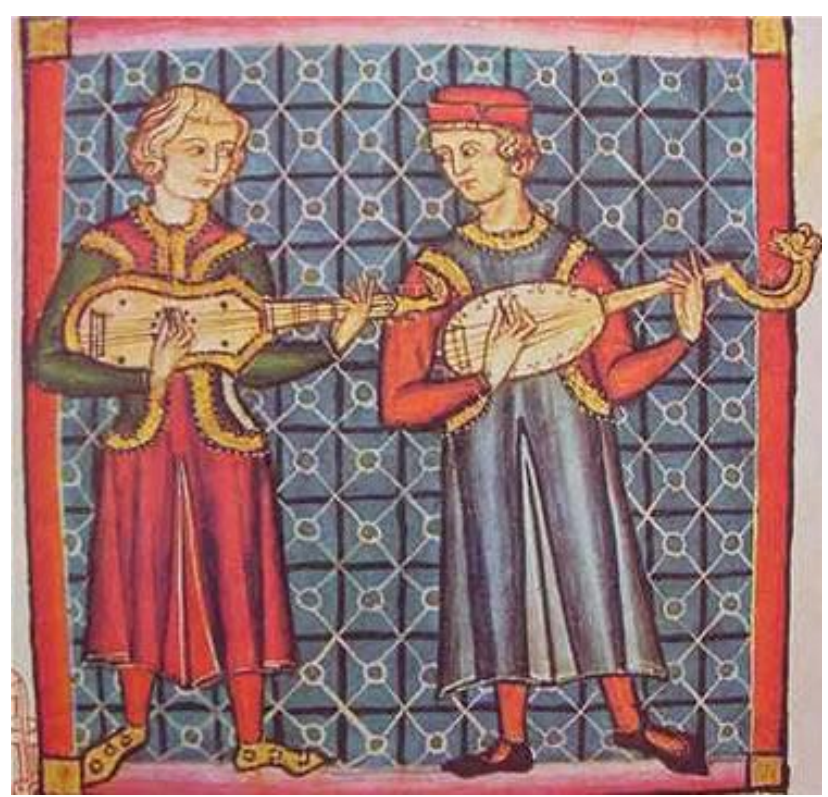

(b)

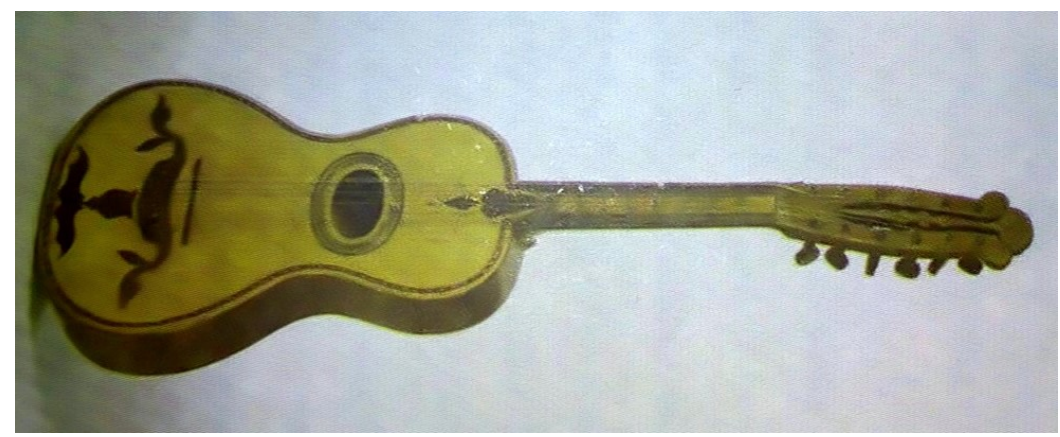

(c)

Figure 1.4: ${ }^{12}$ Precursors of the viola caipira. (a) A typical oud; (b) Medieval painting of Latin guitar and Morisca guitar from the thirteenth century; (c) A typical viola braguesa (extracted from [?]). 
work of the Brazilian Realist painter Almeida Júnior, dated 1899, in which two typical caipiras are depicted, while one of them plays a viola caipira.

Nowadays, the meaning of the term "caipira people" (or just "capira") is quite complex and involves diverse social, cultural and anthropological aspects that will not be discussed in the scope of this thesis. In addition, any attempt to translate this term into other languages could be imprecise and exclusive. In this text, therefore, the terms "caipira" as well as "viola caipira" will be maintained in Portuguese language. It can be stated in a simplistic way, however, that the caipira people are concentrated nowadays in rural or urban areas, mainly in the Center-South region of Brazil. They have cultural traits inherited from the "first caipiras" and traits from the Brazilian urban culture, combining in a continuous and complex way. Many authors have studied different aspects of the caipira people. The interested reader may refer to [?, ?], for example.

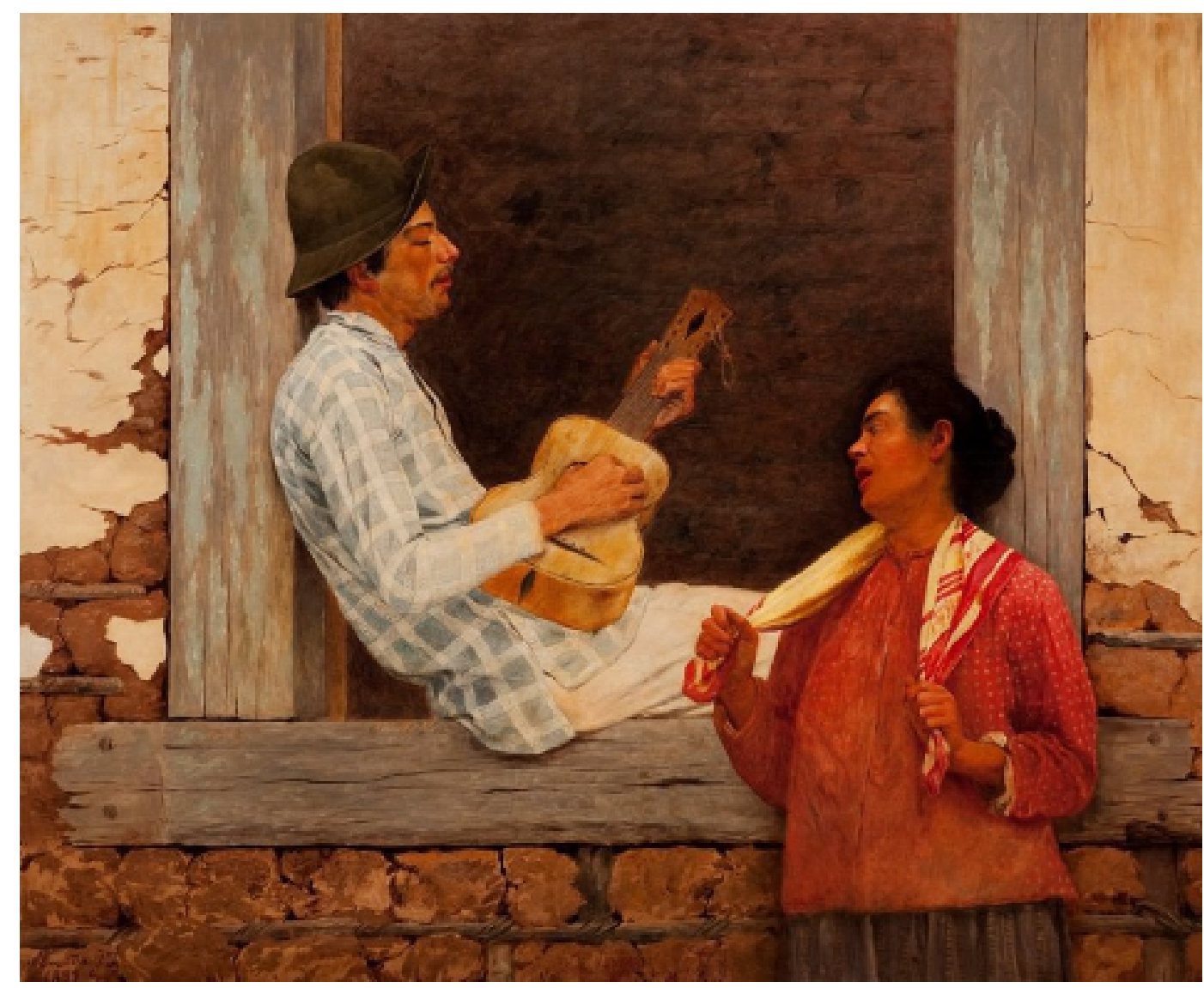

Figure 1.5: Reproduction of the $O$ Violeiro. It was painted in the year 1899 and is currently in the Pinacoteca of the state of São Paulo. It is one of the last works of the São Paulo painter José Ferraz de Almeida Júnior (1850-1899) who sought to insert in several of his paintings aspects of Brazilian regionalism.

The viola caipira is played in numerous activities and ludic-religious manifestations of the Caipira tradition such as the Folia dos Santos Reis, Folia do Divino, the dances of Santa Cruz and São Gonçalo (shown in Figure ??), the Cururu, the Catira or Cateretê, the Moda de Viola, the Quadrilha, the CanaVerde, the Congado, the Moçambique, the Catopé, the Caiapó and others. Martins [?] highlights that the musical dimension represented by the performances, genres and caipira rhythms that accompany such manifestations, is presented as a fundamental element which acts both as a means of prayer and leisure. 
The musical dimension of such practices is denominated caipira music.

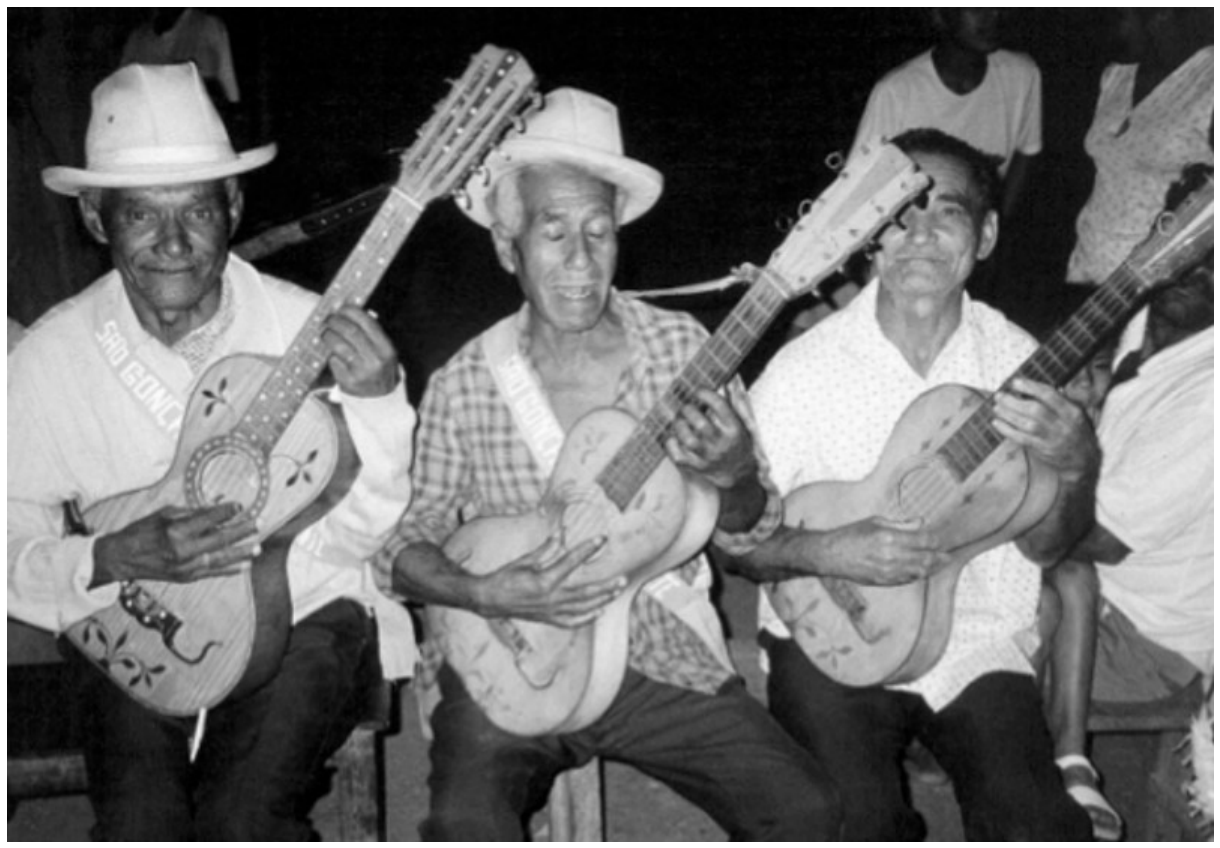

Figure 1.6: Violists in the dance of São Gonçalo, in São Francisco city (Minas Gerais State), in 2000. (extracted from $[?]^{15}$ )

Countless violists contributed and have contributed to the development caipira music as we know it today ${ }^{16}$. To name a few, names like Tião Carreiro, Helena Meirelles, Gedeão da Viola, Zé Coco do Riachão, Índio Cachoeira, Zé Mulato, Bambico, Zézinho da Viola, Antonio Madureira will remain forever in the memory of Brazilian music. Taubkin [?] provides a list of hundreds of violists currently active in Brazil and numerous viola manufacturers.

Although the viola caipira is most often associated to the caipira music, it has been present in many others Brazilian music genres, especially since the mid-1970s . As Vilela [?] points out, important players such as Renato Andrade, Almir Sater, Tavinho Moura, Roberto Corrêa, Paulo Freire, Ivan Vilela, Fernando Deghi, among others, merged in their works the universes of the caipira music and spheres of erudite music, Brazilian popular and instrumental music (MPB) and jazz, each one in different manners, intensities and using different influences. On the musical plurality of the viola caipira and the ways that the instrument has recently taken, Vilela also writes:

There are many violists who played their music all over Brazil. From traditional violists to recording violists, from concert performers like Renato Andrade to the new generations that have emerged since the 1980s. Musicians who merged with the traditional way of playing various elements of their musical backgrounds, namely classical, Brazilian instrumental, folklore, Brazilian Popular Music (MPB), jazz, regional, rock and other trends that have emerged in the musical market in recent decades. Currently, young people from different

15. This photo was taken by Andréa Borghi.

16. For interested readers, the following links are recommended: https : //www.youtube.com $/$ watch $? v=7_{c}$ ecrlsnys (by Almir Sater)

https : //www.youtube.com/watch? $v=-k U Y M T K x n 4 g$ (by Tião Carreiro and Pardinho)

https : //www.youtube.com/watch? $v=n t S-p O m u Z S E$ (by Lucas Reis and Thácio)

https : //www.youtube.com/watch? $=k c y M i 8 m h F E g$ (by Helena Meirelles) 
parts of the country have been playing the viola, leading this instrument to be used in other musical segments in which the viola had never been considered.[?] 


\section{Chapter 2}

\section{StATE OF THE ART}

The study of the physical functioning of a stringed instrument is an essential prerequisite for any proposal of developing sound synthesis models. Regardless of the differences in sound or appearance between plucked or bowed instruments, the principle of sound production of all chordophones are substantially the same. Since the acoustic energy radiated by isolated strings is too weak to be perceived, they are coupled to a radiating element, a role played by the soundboard. It is possible to systematize the functioning of the instrument by means of a so-called functional diagram such as that of Figure ??.

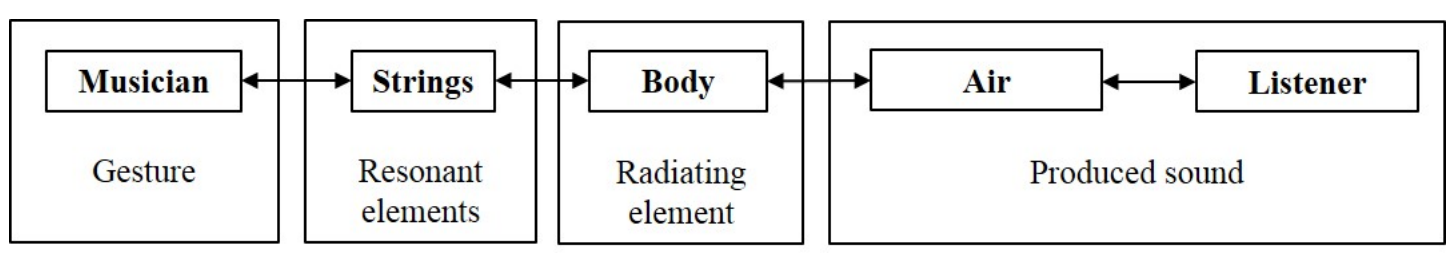

Figure 2.1: Simplified functional scheme of stringed instruments.

This diagram shows two distinct subsystems which constitute the instrument: the strings and the body. The strings play the role of a resonant system since they are the ones that impose the pitch of the note due to the periodic or pseudo-periodic effort that they exert on the bridge. The body is the radiating element since it ensures sufficient acoustic energy for the instrument to be heard.

In the Sachs-Hornbostel organological classification system [?], the viola caipira belongs to the lute family, that is to say, musical stringed instruments composed of a soundboard parallel to the strings plane, a resonance box and strings coupled to the bridge and neck extremity. The neck is used to adjust, by means of the fingers, the length of the strings and consequently the pitch of the note played. Being the viola caipira the focus of this work, the state of the art which follows describes the current knowledge of the various mechanisms involved in the sound production of plucked stringed instruments, in general.

\subsection{The motion of isolated strings}

The vibrating string problem has been studied since a long time. In 1746, D'Alembert [?] stated the one-dimensional wave equation whose solution describes the motion of an ideal string vibrating in a single plane. Ten years later, Euler stated the three-dimensional wave equation and proposed a technique for its solution [?]. The subsequent models are more complex as more physical phenomena are taken into account. 


\section{Ideal plucked strings}

Let us assume an ideal string of length $L$, fixed at both extremities, mass per unit length $\mu$, subject to a constant axial tension $T$. For a small amplitude motion, the string transverse displacement $y(x, t)$, at position $x$ and time $t$, is governed by the one-dimensional wave equation:

$$
\frac{1}{c^{2}} \frac{\partial^{2} y(x, t)}{\partial t^{2}}=\frac{\partial^{2} y(x, t)}{\partial x^{2}},
$$

where

$$
c=\sqrt{\frac{T}{\mu}}
$$

is the velocity of transverse waves xin the string. Since both string extremities are fixed, $y(0, t)=0$, $y(L, t)=0$ and the solution of Equation ?? can be written as the modal superposition:

$$
y(x, t)=\sum_{n=1}^{\infty} a_{n} \sin \left(n \frac{\pi x}{L}\right) \cos \left(n \frac{c t}{L}\right),
$$

where $a_{n}$ is the amplitude of the $n^{\text {th }}$ string mode shape.

Considering a null external force field, a simplified pluck can be described in terms of initial conditions by imposing a triangular shape to the string at the instant of release. Thus, at $t=0$, the string has null velocity and transverse displacement $h$ at the excitation position $x_{0}$ so that its transverse displacement at any point $x$ can be obtained by Fourier series approximation:

$$
y(x, t)=\sum_{n=1}^{\infty} \frac{2 h}{\pi^{2} n^{2} X_{0}\left(1-X_{0}\right)} \sin \left(n \pi X_{0}\right) \sin (n \pi X) \cos \left(n \frac{c \pi t}{L}\right),
$$

where $X_{0}=x_{0} / L$ and $X=x / L$ are the positions normalized by $L$, the string vibrating length. It is worth remembering that this equation is valid for an ideal pluck. In practice, the initial deformation of the string is more complex than a simple triangle. However, several important points can already be noted from Equation ?? as follows:

- Assuming $h$ is weak enough to remain linear, the amplitude of vibration increases as $h$ is large, so that the excitation amplitude is an important parameter.

- The maximum vibration is reached for an excitation point $x_{0}=L / 2$, i.e., at the center of the string.

- The amplitude of the partials globally decreases in $\frac{1}{n^{2}}$.

- The excitation and observation positions lead to "filtering" effects of the overtones.

Concerning the last point above, for certain values of $X$ or $X_{0}$, the terms $\sin \left(n \pi X_{0}\right)$ and $\sin (n \pi X)$ do not contribute the $n^{\text {th }}$ partial and its multiples. For example, if $X_{0}=1 / 2$ (excitation at the center of the string), we have $\sin \left(n \pi X_{0}\right)=\sin \left(\frac{n \pi}{2}\right)$. This term vanishes for every even $n$ and is equal to 1 for every odd $n$. The contributions of the partials of even orders are null because the string is excited at a position corresponding to a node. It is also observed an overall decrease of the amplitudes of partials in $\frac{1}{n^{2}}$ modulated by a "filtering" effect due to the excitation and observation positions. 


\section{Effect of bending stiffness: inharmonicity}

In the ideal string model considered above, the spectrum resulting from a pluck is always composed by harmonic components, whose frequencies are multiples of the fundamental frequency (see, for example, Elie [?], page 16). In practice, the string has a certain stiffness which tends to bring it back to its resting state. The string motion, in this case, is described by including a stiffness term to the wave equation ?? so that

$$
\frac{1}{c^{2}} \frac{\partial^{2} y(x, t)}{\partial t^{2}}=\frac{\partial^{2} y(x, t)}{\partial x^{2}}-\frac{B}{T} \frac{\partial^{4} y(x, t)}{\partial x^{4}},
$$

where $\mathrm{B}$ is the string bending stiffness. The result is an inharmonicity peculiar to freely oscillating strings, which means that the overtone frequencies are not exactly equal to multiples of the fundamental frequency. According to Fletcher [?], the frequency $f_{n}$ of the $n^{\text {th }}$ overtone with respect to the fundamental frequency $f_{0}$ writes

$$
f_{n}=n f_{0} \sqrt{1+B^{\prime} n^{2}}
$$

where $B^{\prime}$ is the inharmonicity factor. It depends on the mechanical and string geometrical parameters [?]:

$$
B^{\prime}=\frac{\pi^{3} E d^{4}}{64 T L^{2}}
$$

where $d$ is the string diameter and $E$ the Young's modulus of its material.

Inharmonicity has often been studied in the cases of guitars and pianos, where tuning problems are common [?, ?]. Inharmonicity factors of different guitar strings were measured experimentally by David [?] and Järveläinen and Karjalainen [?], while for different piano strings they were measured by Fletcher [?], Lieber [?] and Fletcher et al. [?]. Elie ([?], page 18) regrouped and compared all those values. The inharmonicity factors of the guitar strings are generally lower than those of the piano strings.

From a perceptual point of view, Järveläinen et al. have studied our ability to discriminate an inharmonic sound from a perfectly harmonic sound [?, ?]. From their studies, it was found that exists a threshold value of $B^{\prime}$ whose sound is perceived as inharmonic. Below this threshold, inharmonicity is not detectable. This threshold value is frequency-dependent since inharmonicity is perceived more easily at low frequencies than at high frequencies.

Considering the inharmonicity is important for a realistic synthesis. In addition, the inharmonicity factor $B^{\prime}$ depends mainly on the intrinsic parameters of the string, so it is not accessible to a luthier. The mechanical properties of the body, however, can slightly modify the inharmonicity [?, ?]. It is within this framework that the luthier can intervene.

\section{Damping mechanisms intrinsic to the string}

When a string is excited, it is damped over time so that its movement is not perpetual. This phenomenon is due to the transfer of energy from the string to an external system. In the case of stringed instruments, part of the energy of the string is transmitted to the soundboard through the bridge so that the soundboard can radiate. This dissipative mechanism can be called "damping mechanism by coupling". There are also dissipative mechanisms peculiar to the string, which can be called "intrinsic damping mechanisms". The total damping of the string is then given by the sum of the terms of intrinsic mechanisms and the term due to the coupling with body. Valette and Cuesta [?] provide a complete review of the damping mechanisms intrinsic to an isolated string. A summary is given below. 


\section{The air viscosity}

Considering the string as a cylinder oscillating at a certain frequency in a viscous fluid, a mechanical resistance leads to energy losses. The expression to calculate the mechanical resistance due to the air viscosity is given by Stokes [?]:

$$
R^{\prime}=2 \pi v_{\text {air }}+2 \pi d \sqrt{\pi v_{\text {air }} \rho_{\text {air }} f}
$$

where $v_{\text {air }}$ and $\rho_{\text {air }}$ are the air dynamic viscosity and density, respectively. The contribution of the air viscosity to the $n^{\text {th }}$ string mode is given by the $Q$-factor[?]:

$$
Q_{n, \text { air }}^{-1}=\frac{R^{\prime}}{2 \pi \mu} \frac{1}{f_{n}}
$$

\section{The visco and thermoelasticity}

The visco and thermoelastic losses are generally represented by the addition of their respective loss angles, $\delta_{V E}$ and $\delta_{T E}$, to the Young's modulus written in the complex form:

$$
\tilde{E}=E\left(1+i \delta_{V T}\right),
$$

where $\delta_{V T}=\delta_{V E}+\delta_{T E}$. For metal, the loss angles are generally considered as constant. The $Q$-factor associated to the $n^{\text {th }}$ mode due to the visco and thermoelastic losses is given by[?]:

$$
Q_{n, V T}=\frac{4 \pi^{2} E I}{T^{2} c}\left(\delta_{V E}+\delta_{T E}\right) f_{n}^{2}
$$

\section{The dry friction in wound strings}

In wound strings another dissipation mechanism should be taking into account due to the dry friction between two successive turns of wire. This mechanism is modelled by the addition of a loss angle $\delta_{W}$ in the term of string tension, which is written in the complex form

$$
\tilde{T}=T\left(1+i \delta_{W}\right)
$$

so that the the $Q$-factor associated to the $n^{\text {th }}$ mode is given by $Q_{W}^{-1}=\delta_{W}$.

\section{The dislocation phenomenon}

In order to consider the damping mechanism due to the dislocation phenomenon in the string material [?], Cuesta [?] incorporates the term $Q_{\text {disl. }}^{-1}$ to the string total damping. Note that this term is frequency-independent over the audio frequency range.

\section{String damping models}

Valette and Cuesta [?] propose a damping model for an isolated string taking into account the abovepresented damping mechanisms. The total expression for the modal $Q$-factor of an isolated string is then given by

$$
Q_{n}^{-1}=Q_{n, a i r}^{-1}+Q_{n, V T}+Q_{\text {disl. }}^{-1}
$$


where the the terms in the right-hand side are respectively related to the air viscosity, visco and thermoelastic and dislocation losses. For wound strings, the term $Q_{W}^{-1}$ is added to Equation ??

The identification of all the parameters of a damping model is long and tedious. A more pragmatic approach has been proposed by Woodhouse in [?]. The determination of the loss factor $\eta_{n}=Q_{n} / 2$, associated to the $n^{\text {th }}$ string mode is given by:

$$
\eta_{n}=\frac{T\left(\eta_{F}+\eta_{A} / \omega_{n}\right)+B \eta_{B}(n \pi / L)^{2}}{T+B(n \pi / L)^{2}}
$$

where $\eta_{A}, \eta_{B}$ and $\eta_{F}$ are terms associated respectively to the losses due to the air viscosity, bending stiffness and either friction between string turns, in wound strings, or inter-molecular effects, in flat strings. Concerning the nature of such approach, Woodhouse summarizes:

This approach should be understood as a combination of physically-based modelling and curve fitting, since the physical mechanisms are not understood in sufficient detail to provide a fully convincing predictive model. ([?], page 956)

\subsection{String/body coupling}

The effects of strings/bridge coupling on the string dynamics have been studied by several authors. Focusing on the violin family instruments, Raman [?] and Schelleng [?] investigated the coupling between string and body resonances and found that the resonant response of a string is significantly perturbed when it is strongly coupled to the main body resonance, which produces the well-known wolf-note (refer to [?], Chapter 25, for a detailed discussion). In addition, Gough [?] developed an analytic two-polarization model able to predict interactions between sympathetically tuned strings through the bridge motion.

The bridge motion also affects the motion of the string by inducing degeneracy breaking of the two transverse polarisations, which leads to beating tones and two-stage decay rates in the sound produced. These phenomena result from the bridge action in the two polarizations [?].

Several works attempted to model and investigate the effects of the multiple-strings/bridge coupling in pianos [?, ?, ?], whose strings are organized in pairs or triplets; sitars [?, ?, ?] and tanpuras [?, ?], Indian musical instruments where strings/bridge contact is quite peculiar [?], Portuguese guitars [?] and harps $[?, ?, ?, ?]$.

In order to model string/body interactions, Woodhouse [?] proposed two approaches for the pluck response synthesis of classical guitars: one is carried out in the frequency domain using transfer functions and other uses modal superposition of string/body coupled modes. Although completely different, these approaches start from the same information: input body admittance measured or simulated at the bridge and string properties. Both methods are briefly described below. Finally, the effects on the string vibration due to the coupling with the body are reviewed.

\subsubsection{String/body coupling using transfer functions}

This method allows to compute the string velocity resulting from a pluck based on the fact that at the coupling point, the string and bridge velocities are identical and the total force exerted is the sum of the forces applied to the two separate subsystems. Thus, it follows the relation

$$
Y_{\text {coup. }}^{-1}=Y_{\text {string }}^{-1}+Y_{\text {bridge }}^{-1}
$$


where $Y_{\text {coup. }}, Y_{\text {string }}$ and $Y_{\text {bridge }}$ represent respectively the coupled system admittance, the string admittance and the bridge admittance at the string/body coupling point. The bridge admittance $Y_{\text {bridge }}$ is easily accessible by measurement since it is defined in the frequency domain as the ratio between the velocity response $V(\omega)$ and the force $F(\omega)$ applied at the same point. The string admittance is however more difficult to measure, so its analytic expression is derived from the string properties. The velocity response $G=\frac{\dot{y}\left(x_{p}\right)}{F(L)}$, given at the plucking point $x=x_{p}$ due to a force applied at the string/body coupling point $x=L$ is then computed by multiplying $Y_{\text {coup }}$. the dimensionless transfer function

$$
H=\frac{y(L)}{\bar{y}(x)},
$$

where $y(L)$ and $\bar{y}(x)$ are respectively the displacement applied at $x=L$ and the corresponding displacement at $x=x_{p}$. An analytical expression for $H$ is given by [?]. Making use of the reciprocity principle, one may obtain

$$
G=Y_{\text {coup. }} . H=\frac{\dot{y}\left(x_{p}\right)}{F(L)}=\frac{\dot{y}(L)}{F\left(x_{p}\right)},
$$

where $\dot{y}\left(x_{p}\right)$ and $F(L)$ denote respectively the string velocity taken at $x_{p}$ and the impulse force applied at the string extremity, at $x=L$. The time-domain velocity $g$ is finally obtained by calculating the inverse Fourier transform of $G$.

\subsubsection{String/body coupled modes computation}

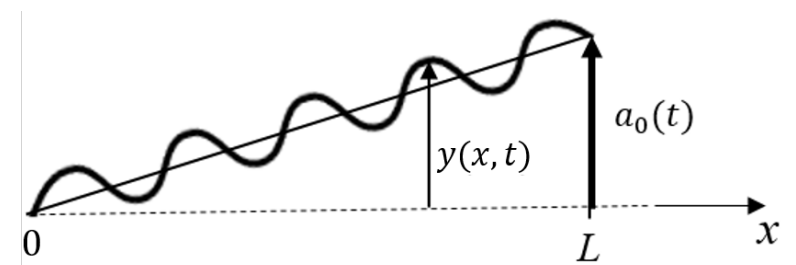

Figure 2.2: Representation of the kinematics of one string coupled to the body assuming a single plane motion. For reasons of clarity of the figure, the amplitude of $a_{0}(t)$ has been shown intentionally higher than the amplitude of $y(x, t)$.

This method derives from substructuring analysis [?] and starts from the kinematics description of string and instrument body separately. The string displacement is given by the sum of the $N_{s}$ modes associated to the string with simply supported ends to which is added one "constraint mode" whose shape $\phi_{0}^{s}(x)=\left(\frac{x}{L}\right)$ corresponds to the static response of the string when the end at the coupling point $x=L$ is allowed to move. Accordingly, the string transverse displacement $y(x, t)$ writes:

$$
y(x, t)=\sum_{n=1}^{N_{s}} \sin \left(\frac{n \pi x}{L}\right) a_{n}(t)+\phi_{0}^{s}(x) a_{0}(t),
$$

where $a_{0}(t)$ is the modal displacement associated to the "constraint mode" (see Figure ??). On the other hand, the body transverse displacement at the coupling point is described as the sum of the $N_{b}$ modes:

$$
y^{b}(L, t)=\sum_{k=1}^{N_{b}} \phi_{k}^{b}(L) b_{k}(t),
$$


where $\phi_{k}^{b}(L)$ and $b_{k}(t)$ are respectively the shape at the coupling point and the amplitude of the $k^{\text {th }}$ body mode. The use of the "constraint mode" makes the string/body coupling possible since both string and body have identical displacements at the coupling point so that $a_{0}(t)=y^{b}(L, t)$. Thus, the string displacement at a generic position $x$ writes

$$
y(x, t)=\sum_{n=1}^{N_{s}} \sin \left(\frac{n \pi x}{L}\right) a_{n}(t)+\phi_{0}^{s}(x) \sum_{k=1}^{N_{b}} b_{k}(t) \phi_{k}^{b}(L) .
$$

The governing equations of the coupled system is then formulated as a set of $N=N_{s}+N_{b}$ secondary-order ordinary differential equations

$$
\mathbf{M} \ddot{\mathbf{q}}(t)+\mathbf{C} \dot{\mathbf{q}}(t)+\mathbf{K q}(t)=\mathbf{f}(t),
$$

where $\mathbf{q}(t)=\left(a_{1}(t), \ldots, a_{N_{s}}(t), b_{1}(t), \ldots, b_{N_{b}}(t)\right)^{T}$ is the vector containing string and body modal displacements; $\mathbf{M}, \mathbf{C}$ and $\mathbf{K}$ are respectively the mass, damping and stiffness matrices; and $\mathbf{f}$ is the column vector containing the modal forces. The vector $\mathbf{q}(t)$ is the only unknown of the problem. Recasting the system of equations ?? into the first-order form, the modes of the string/body coupled system are computed and superposed to construct the string response to a given excitation. For further details the reader is invited to refer to [?].

\subsubsection{Coupling effects on the string modes}

When the string end at $x=L$ is coupled to a moving structure like the guitar body, the latter perturbs the movement of the string, and in particular its wave number. This perturbation can be satisfactorily described as the addition of a small term $\delta_{n} \ll 1$ to the wave number of the isolated string [?]. By making use of the continuity condition between string and bridge, more specifically, imposing the equality of the admittances $Y_{\text {string }}$ and $Y_{\text {bridge }}$ at the coupling point $x=L$, Pate et al [?] ${ }^{1}$, inspired by Valette and Cuesta [?], show that the modal frequencies and modal Q-factors of the isolated string are perturbed by the imaginary part (susceptance) and real part (conductance) of $Y_{\text {bridge }}$, respectively, so that

$$
\bar{f}_{n}=\frac{j c}{2 L}\left[1+\frac{n^{2} \pi^{2}}{L^{2}} \frac{E I}{2 T}+\frac{Z_{c}}{n \pi} \mathfrak{J}\left(Y_{\text {bridge }}\left(L, f_{n}\right)\right)\right]
$$

and

$$
\bar{Q}_{n}^{-1}=Q_{n}^{-1}+\frac{c^{2} \mu}{\pi L} \mathfrak{R}\left(Y_{\text {bridge }}\left(L, f_{n}\right)\right) \frac{1}{f_{n}},
$$

where $\bar{f}_{n}$ and $\bar{Q}_{n}$ are the modal frequencies and the modal Q-factors of the string coupled to the body and $Z_{c}=\sqrt{\mu T}$ is the characteristic impedance of the string.

It is expected, therefore, that the imaginary part of the bridge admittance affects the modal frequencies of the string and consequently its inharmonicity [?]. However, measured admittance imaginary parts on the electric [?] and acoustical [?] guitars never lead to a frequency shift larger than $1 \mathrm{~Hz}$.

The total Q-factor associated to the damping of the $n^{\text {th }}$ overtone is the sum of two terms: a term intrinsic to the string, given by Equation ??, which includes all energy dissipation mechanisms (viscoelastic losses, visco-thermal, internal frictions, etc.), and a term relative to the coupling with the instrument body, given by the second term in the right-hand side of Equation ??. This relationship reveals the compromise that the luthier has to face: a high level of bridge admittance ensures a certain sound

1. In this work, Paté et al study the solid body electric guitar, where the string/body coupling occurs mainly via the fingerboard so that $Y_{\text {bridge }}$ is neglected and the fingerboard admittance is included. 
power but reduces the duration of sound. Moreover, since the bridge admittance varies with frequency, the damping due to the coupling may considerably vary from note to note.

In scientific terms, the art of the luthier consists of adjusting the duration and intensity of sound by modifying the level of bridge admittance. To ensure a certain homogeneity in terms of sound duration along the instrument frequency range, this adjustment sometimes is made locally. In this sense, this work focuses more on the damping mechanism by coupling since it is somehow controllable by the luthier, while the other terms are solely dependent on the intrinsic characteristics of the string.

In order to include all above-presented damping mechanisms to the string motion, i. e., coupling and string-intrinsic effects, Valette and Cuesta [?] took the damping of each "inharmonic" overtone $\bar{f}_{n}$ into account by introducing "corrected" modal Q-factors in the general solutions of the string equation ??:

$$
y(x, t)=\sum_{n=1}^{\infty} a_{n}(t) \sin \left(\frac{n \pi x}{L}\right) \sin \left(2 \pi \bar{f}_{n} t\right) e^{-\frac{\pi \bar{f}_{n}}{\bar{Q}_{n}} t} .
$$

\subsection{Vibroacoustical behavior of the body}

\section{Experimental modal analysis}

Since the soundboard acts as a radiating element (cf. Figure ??) in the sound production of guitarlike instruments, its vibratory behaviour is determinant on the sound resulting from a note played by the musician. Thus, numerous studies deal with the vibratory properties of the soundboard, coupled or not with the rest of the entire guitar.

The shapes of the first guitar modes shown in Figure ?? reveal that the main vibrating region of the instrument resonance box is located on the lower bout of the soundboard, under the rosette, being the upper bout region very little mobile. In general the frequencies of these first modes vary little from one guitar to another due to the small variation in the instrument geometry. 


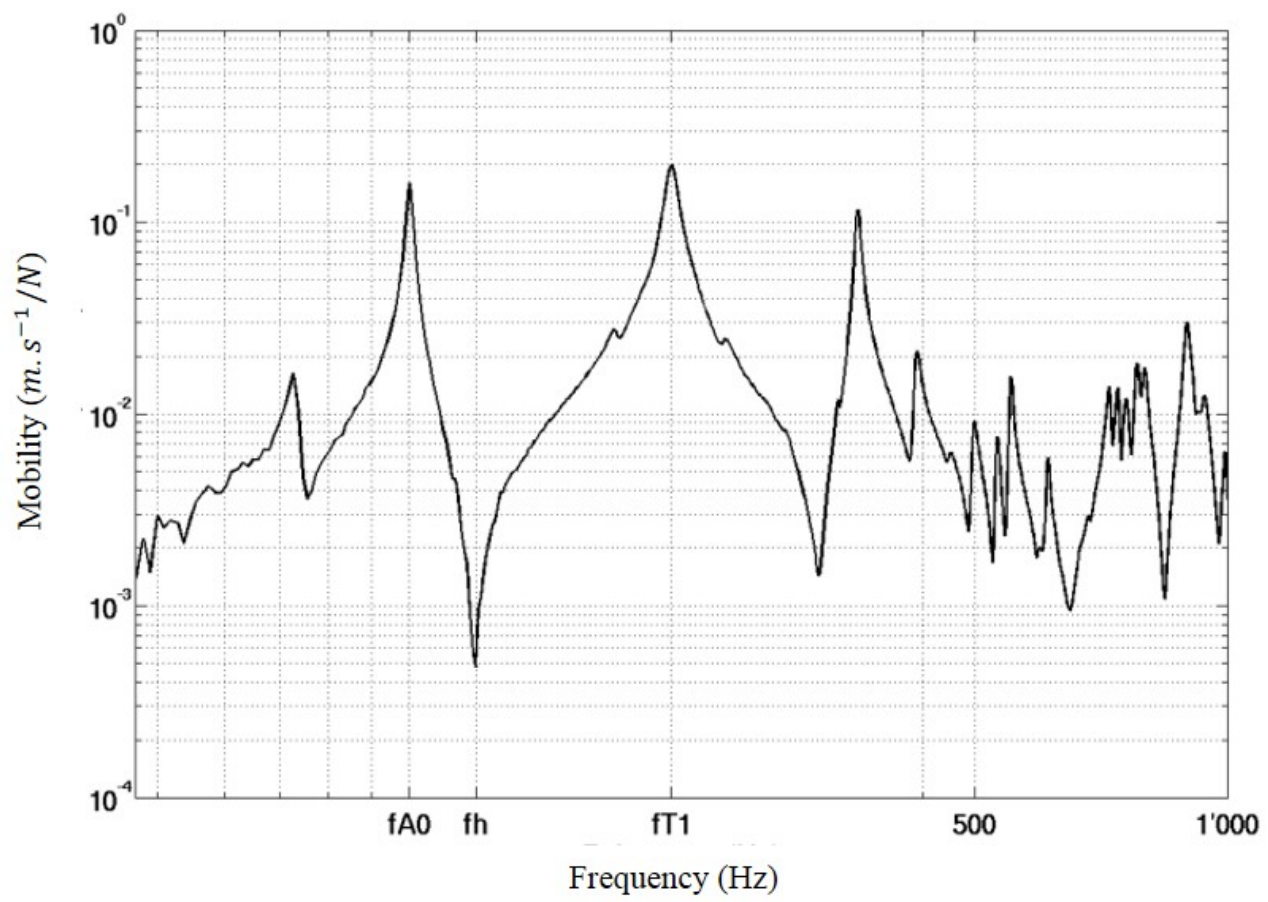

Figure 2.3: Example of frequency response function measured at the bridge of a classical guitar. The characteristic frequencies are indicated with their usual symbols. Extracted from [?].

Jansson [?] was the first to publish images of guitar mode shapes, which were obtained by means of hologram interferometry. Firth [?] then applied the Chladni technique to determine the nodal lines of the 8 first soundboard modes and 9 first back plate modes. In order to classify and specify the origin of some of these modes, specific names have been assigned according to the nomenclature adopted by him [?]:

- The first mode, denoted by $A_{0}$, is also called the "air cavity mode" and corresponds to the predominant motion of the mass of air inside the instrument cavity coupled to the moving walls of the resonance box (soundboard, back plate and sides). For classical guitars, in general, the frequency $f_{A_{0}}$ is systematically located at around $90-100 \mathrm{~Hz}$ [?].

- The first soundboard mode, denoted by $T_{1}$, is associated to considerable motion of the soundboard coupled to the air cavity motion. Its frequency $f_{T_{1}}$ is located at around $190-220 \mathrm{~Hz}$ for most classical guitars [?].

It is worth mentioning that other instruments have similar characteristics, in particular the concert [?] and Gothic harp [?], which also feature modes $A_{0}$ and $T_{1}$, but in different frequency ranges.

Another characteristic frequency is the Helmholtz frequency, commonly denoted by $f_{h}$. It is "purely" associated to the motion of the air cavity without coupling to the instrument structure. In guitars, it appears as an antiresonance in the frequency response function measured at the bridge of the instrument, as shown in Figure ??. The first peak, at around $75 \mathrm{~Hz}$, just before the $A_{0}$ mode, is a "global mode", corresponding to an overall bending motion of the whole structure of the instrument. There is very little documentation on this mode; Jansson [?] refers to a "bending motion of the complete guitar", while Meyer [?] classify it as a "secondary resonance". Figure ?? shows the first three mode shapes obtained by Hill and Richardson [?] for a classical guitar using holographic interferometry. 


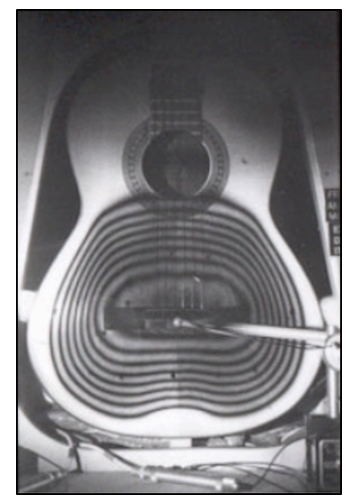

(a) $A_{0}(94.5 \mathrm{~Hz})$

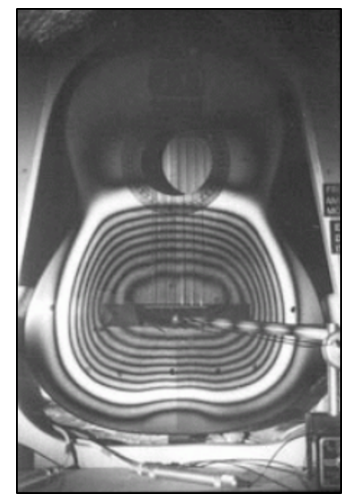

(b) $T_{1}(182.5 \mathrm{~Hz})$

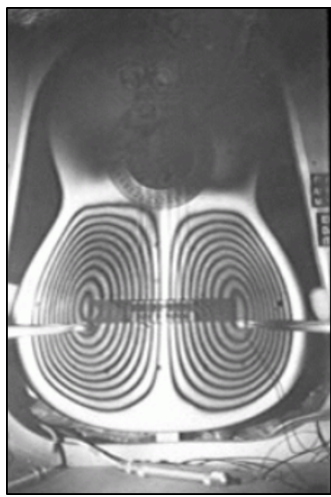

(c) $T_{2}(218 \mathrm{~Hz})$

Figure 2.4: First three mode shapes of a classical guitar, extracted from[?].

\section{Simple guitar models with few degrees of freedom}

In order to understand in a simple way the role of the low-frequencies in the guitar sound production, some authors have proposed discrete models to study the coupling between the soundboard and the air mass inside the cavity of the guitar. In the late 1970s, Firth [?] and Caldersmith [?] proposed modelling the cavity as a bass-reflex enclosure. Firth proposed an equivalent electrical circuit describing the mechanical behaviour of the guitar in the vicinity of the frequencies of the modes $A_{0}$ and $T_{1}$. This circuit is very similar to a bass-reflex enclosure. The author concludes that the role of the air cavity is essentially to amplify the response of the instrument in low frequencies. Using the geometrical data of the guitar, as well as the mechanical properties of its materials, the Caldersmith model [?] allows to predict the frequencies and quality factor of the modes $A_{0}$ and $T_{1}$.

In 1980, Christensen and Vistisen [?] proposed a discrete model with 2 degrees of freedom (DOF) to study the coupling between the soundboard and the air cavity (see Figure ??). The soundboard is replaced by a piston of area $A_{p}$ and mass $m_{p}$, connected to two springs, one describing the stiffness of the plate, denoted by $k_{p}$, and the other, not shown in Figure ??, describing the coupling with the fluid domain. $m_{h}$ denotes the mass of the air in the vicinity of the rosette represented by an air piston of area $A_{h}$ oscillating against the stiffness of the air volume inside the cavity. The coupled motion of the soundboard and the air cavity gives rise to the modes $A_{0}{ }^{2}$ and $T_{1}$, which correspond respectively to a predominant air cavity motion coupled with the soundboard and a predominant soundboard motion loaded by the cavity. The antiresonance frequency $f_{h}$ observed between $f_{A_{0}}$ and $f_{T_{1}}$ corresponds to the resonant frequency of the Helmholtz resonator associated to the rigid-wall air cavity motion. The frequencies $f_{A_{0}}, f_{T_{1}}$ and $f_{h}$ are linked to the frequency $f_{p}$ through the stiffness of the air in the cavity so that the following relation is given:

$$
f_{p}^{2}+f_{h}^{2}=f_{A_{0}}^{2}+f_{T_{1}}^{2}
$$

This model has the advantage of allowing an estimation of characteristic parameters of the guitar from the simple measurement of the frequency response on the soundboard. Indeed, the values of $f_{A_{0}}, f_{T_{1}}$ and $f_{h}$ can be extracted directly from the frequency response curve of the instrument (see, for example, Figure ??). It is then possible to calculate the frequency $f_{p}$ from Equation ??. However, in terms of

2. The term "Helmholtz resonance" is sometimes applied to the $A_{0}$ mode, but this resonance involves considerable motion of the soundboard and back plate and so is not a simple Helmholtz cavity resonance.[?] 


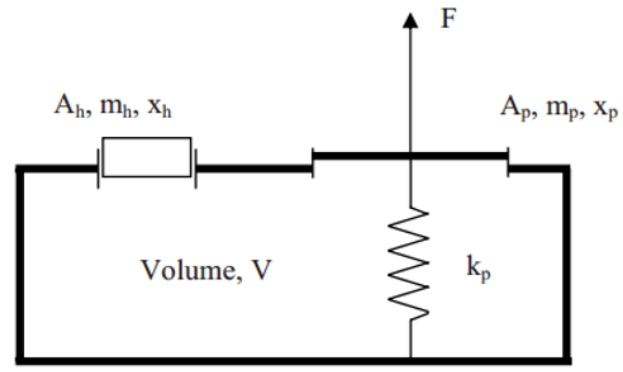

(a) 2-DOF model.

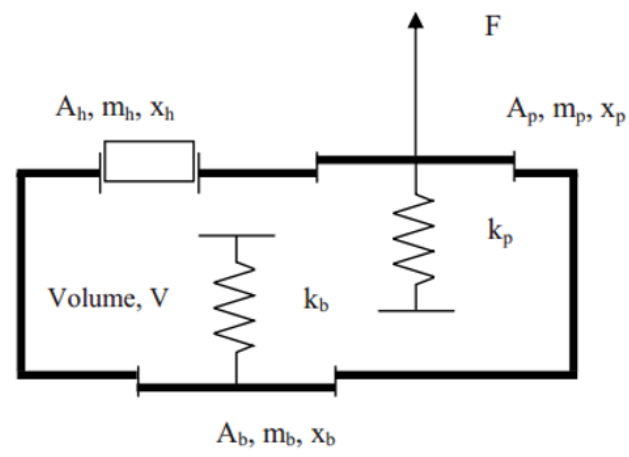

(b) 3-DOF model.

Figure 2.5: Schemes for guitar models with 2 (a) and 3 (b) DOF (extracted from [?]).

frequency range, this model is very limited since it only describes the behaviour of the first two modes of the instrument.

A natural extension of this model is to add a third degree of freedom, constituted by an additional mass representing the back plate of the guitar. This model was proposed by Christensen [?] and experimentally validated by Rossing et al. [?], and later extended by Wright [?], who coupled strings to the instrument body using the theory presented by Gough [?]. The frequencies $f_{A_{0}}, f_{T_{1}}, f_{d}, f_{h}$ and $f_{b}$ are then linked together by the relation

$$
f_{p}^{2}+f_{h}^{2}+f_{b}^{2}=f_{A_{0}}^{2}+f_{T_{1}}^{2}+f_{d}^{2},
$$

where $f_{d}$ is the frequency of the mode associated to the motion of the back plate coupled with the whole structure and air cavity, and $f_{b}$ is the second antiresonance, located between $f_{T_{1}}$ and $f_{T_{2}}$.

Popp [?] recently proposed a 4-DOF model, which includes the sides. The modifications of the boundary conditions imposed by the way that the musician hold the instrument can thus be taken as input parameters of the model.

\section{Soundboard radiation}

The study of the guitar radiation begins with Lai and Burgess [?], published in 1990. The authors use the same method employed by Suzuki [?] for piano soundboards to obtain the radiation efficiency of different acoustic guitars. The method consists of exciting the guitar at its bridge using a shaker and measuring the accelerance at the driving point and the sound intensity in the near field. The former measurement is obtained by means of an impedance head; the latter, with a sound-intensity probe. The radiation efficiency is then determined from the ratio of the soundboard radiated sound power and the input power. It is shown in Figure ?? that the $A_{0}$ mode is mainly related to a dominant sound radiation in the region of the sound hole.

Boullosa et al. [?] proposed establishing a relationship between the magnitude of the total radiation efficiency of a guitar and its perceptual evaluation. The total radiation efficiency is defined as:

$$
E_{\text {Total }}=\frac{\sum_{n=1}^{N} E_{n} \Delta f_{n}}{\sum_{n=1}^{N} \Delta f_{n}}
$$


where $E_{n}$ is the efficiency in band $n$ and $\Delta f_{n}$ is the bandwidth of the corresponding one-third octave band. From tests on four guitars, the authors conclude that the guitars with the greatest radiation efficiency are those that are judged most positively, and that therefore the radiation efficiency plays somehow an important role in the subjective appreciation of the instrument.

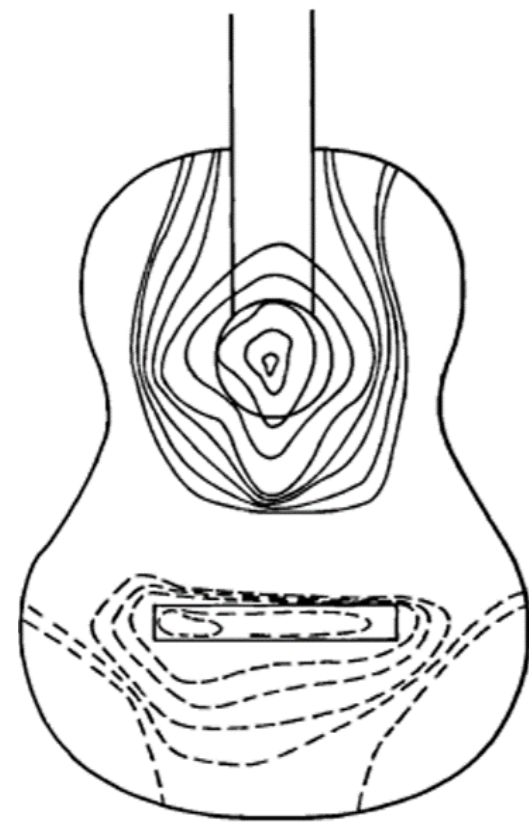

(a) $A_{0}(91 \mathrm{~Hz})$

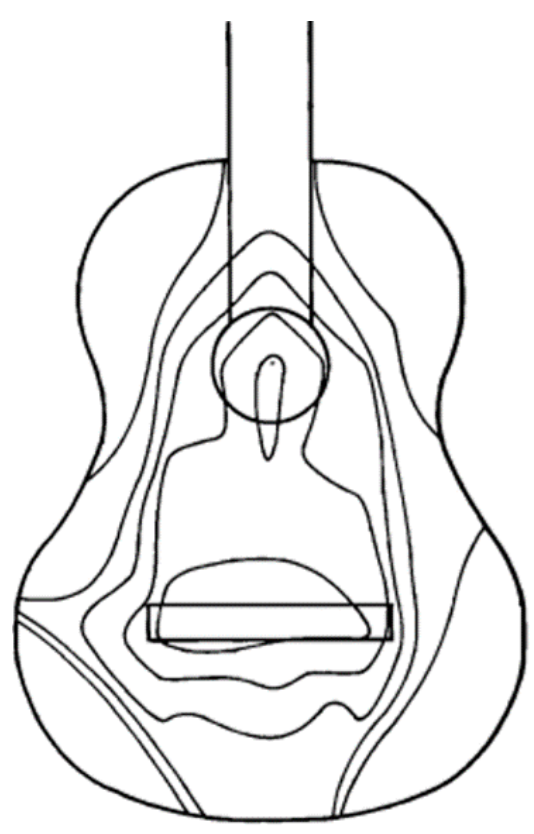

(b) $T_{1}(189 \mathrm{~Hz})$

Figure 2.6: Sound intensity contours for a classical guitar at the two first modes [?]. Dashed lines and lines represent positive and negative countour values, in $\mathrm{dB}$, respectively.

Christensen [?] extended his 2 and 3-DOF guitar models by coupling a moving piston to each oscillator representing a degree of freedom. Each piston acts as a source of monopole radiation so that the guitar sound pressure level is obtained by the superposition of the contributions from each oscillator. This study allows to model the acoustic response function of guitars up to $600 \mathrm{~Hz}$ using only the parameters of the first modes. More recently, Hill et al. [?] improved this model taking into account the radiation directivity of each piston. The three-dimensional diagrams of the radiated acoustic field for the first 3 modes of the guitar and respective shapes obtained by holographic interferometry are shown in Figure ??. 


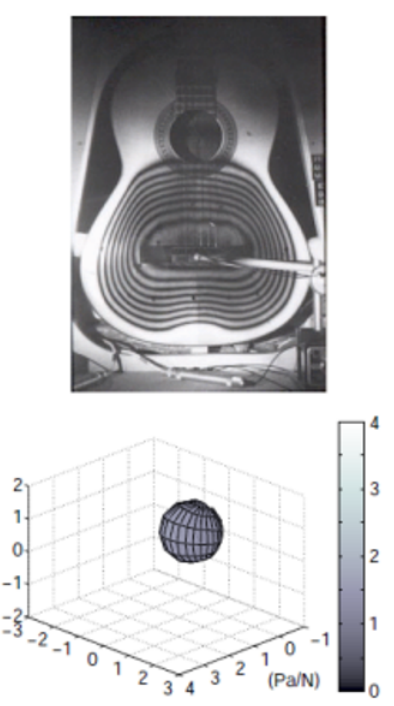

(a) $A_{0}$

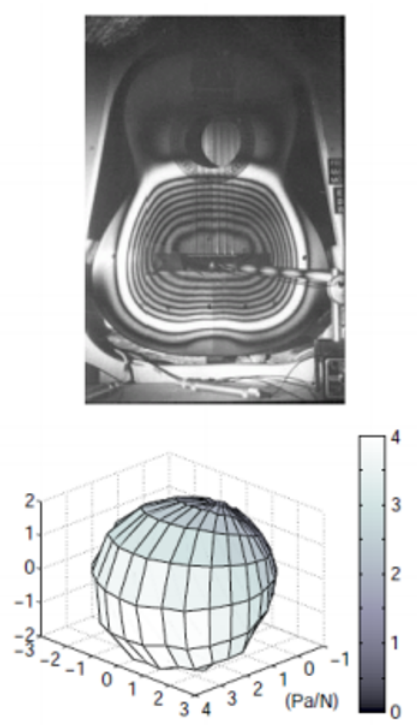

(b) $T_{1}$
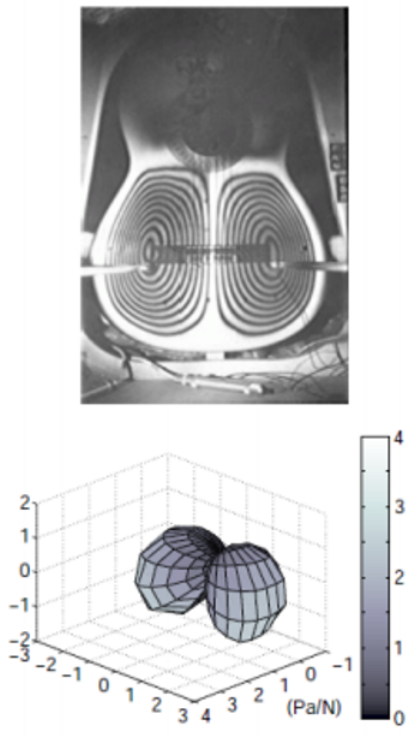

(c) $T_{2}$

Figure 2.7: Mode shapes and 3D diagrams of the radiated acoustic field for the three first classical guitar modes [?].

\subsection{Finite difference schemes in sound synthesis}

As previously mentioned, the displacement of a simply supported isolated string in time and space domains can be described by the partial differential equation ?? (PDE), whose general solution is given analytically by the modal superposition in Equation ??. However, Equation ?? becomes more complex as additional terms describing, for example, damping mechanisms, string terminations, bending stiffness or excitation mechanisms, are incorporated to conveniently model the instrument. In such cases, analytical solutions are normally no longer available so that numerical solution methods have to be employed.

In musical instrument sound synthesis, the most used and straightforward method for solving PDEs makes use of finite differences approximations [?, ?]. The initial idea of this method consists of discretizing the spatial and temporal domains into a finite set of points and approximating derivatives by differences between the values at nearby points. Substituting in the PDE the derivatives by their respective approximations, a recursion formulae is then derived, which allows to compute the solution along the grid of points in space and time. Compared to other solution methods such as digital waveguides [?, ?] or modal synthesis $[?, ?, ?]$, one of the main advantages of the finite difference method is its versatility, since it can be applied to different systems, including those strongly non-linear. On the other hand, computational cost and problems of numerical instability are points that deserve special attention when such a method is employed.[?]

In 1969, Ruiz[?], followed by others [?, ?, ?, ?], pioneered the application of finite differences schemes to solve PDEs and consequently obtain synthesized musical sounds. These works marked a first important stage in the development of the string instruments synthesis, despite the computers at that time did not have the required processing power to properly run the involved calculations. About 20 years later, Chaigne et al. [?] use a central finite difference schemes, which are described in detail in his previous 
work with Guyard [?], to discretize string motion equations taking into account string characteristics (material, tension and geometry), boundary conditions (impedances at the bridge and stopping finger) and excitation mechanisms (finger pluck, hammer strike, etc). They obtained close agreement between numerically simulated and measured guitar sounds. In a subsequent work, Chaigne and Askenfelt [?, ?] use a similar numerical approach to obtain synthesized piano sounds with a "high degree of realism". In comparison with previous related studies, their physical model, as they themselves describe in the paper, "is entirely based on finite difference approximations of the continuous equations for the transverse vibrations of a damped stiff string struck by a non-linear hammer". Giordano and Jiang [?] considerably extended this model by dealing with a complete model composed of three submodels: string and hammer interactions and their respective motions, soundboard vibrations and sound production by the vibrating soundboard.

Finite difference methods have been used in numerous different approaches to synthesize the sound of various instruments. By way of example, several works and respective addressed instruments can be listed, such as xylophone [?], kettledrums [?], snare drum [?, ?], timpani [?], cymbal and gong [?], harp [?], tanpura [?, ?], guitar [?, ?, ?], electric bass [?, ?], Portuguese guitar [?] and violin [?, ?, ?, ?]. In addition, Portnoff [?] contributed significantly to the context of vocal tract and speech synthesis. Finally, we cannot fail to mention the Bilbao's book [?], in which is presented a thorough study of finite difference schemes applied to various objects in the musical acoustics context including strings, beams, plates, as well as coupled objects such as prepared strings, coupled beams, string-plate coupling and transverselongitudinal coupling in strings.

\subsection{Previous works on the viola caipira}

As previously mentioned, the viola caipira is an instrument little explored from the musical acoustics perspective. The literature review performed for this research revealed that the only work addressing the viola caipira in this field is the master dissertation [?] written by this author in 2012, which resulted in the publication of two conference papers [?, ?]. The following is a brief summary of the results obtained in these works.

In [?] is presented a set of finite element analyses of three models of the viola caipira resonance box without strings and neck. These models have geometries with different detail levels: the first model adopts approximate dimensions of a real viola caipira and neglects the internal reinforcements (struts, ribs, bars, etc); the second model is an extension of the first model with internal reinforcements; the third model is rigorously designed according to the dimensions of a real viola caipira, including internal reinforcements. Three types of modal analysis are performed for each model. Acoustical modes associated to the air cavity inside the resonance box as well as the structural ones are determined in terms of natural frequencies and mode shapes. Structure and air cavity are then coupled and vibroacoustical modes are determined. Experimental mode shapes and natural frequencies are obtained using the Chladni technique so that the simulated results are finally validated.

It is observed that the inclusion of internal reinforcements leads to significant increase of the natural frequencies (from $23 \%$ to $43 \%$ ) and considerable change of the mode shapes, mainly from the fourth mode, whose frequency is $280.68 \mathrm{~Hz}$. This is expected since the first three modes are strongly determined by the geometry of the instrument box [?]. On the other hand, it has been shown that the coupling with the air cavity leads to the emergence of new modes and affects both frequency and mode shapes of all modes in general. Figure ?? shows a comparison of the five first acoustical and structural modes of the viola caipira with those obtained for the classical guitar by other authors. Note that the order of the acoustical modes A3 (fourth mode) and A4 (fifth mode) is reversed, that is, the fourth mode of the classical guitar 
corresponds to the fifth mode of the viola caipira, and vice versa. In addition, it can also be seen that in general the structural and acoustical mode shapes of both instruments are very similar. Note that, for both instruments, the second structural mode, denoted by $\mathrm{B}(1,1)$, is characterized by the predominance of the back plate vibration, while for the other modes, the soundboard vibration prevails.

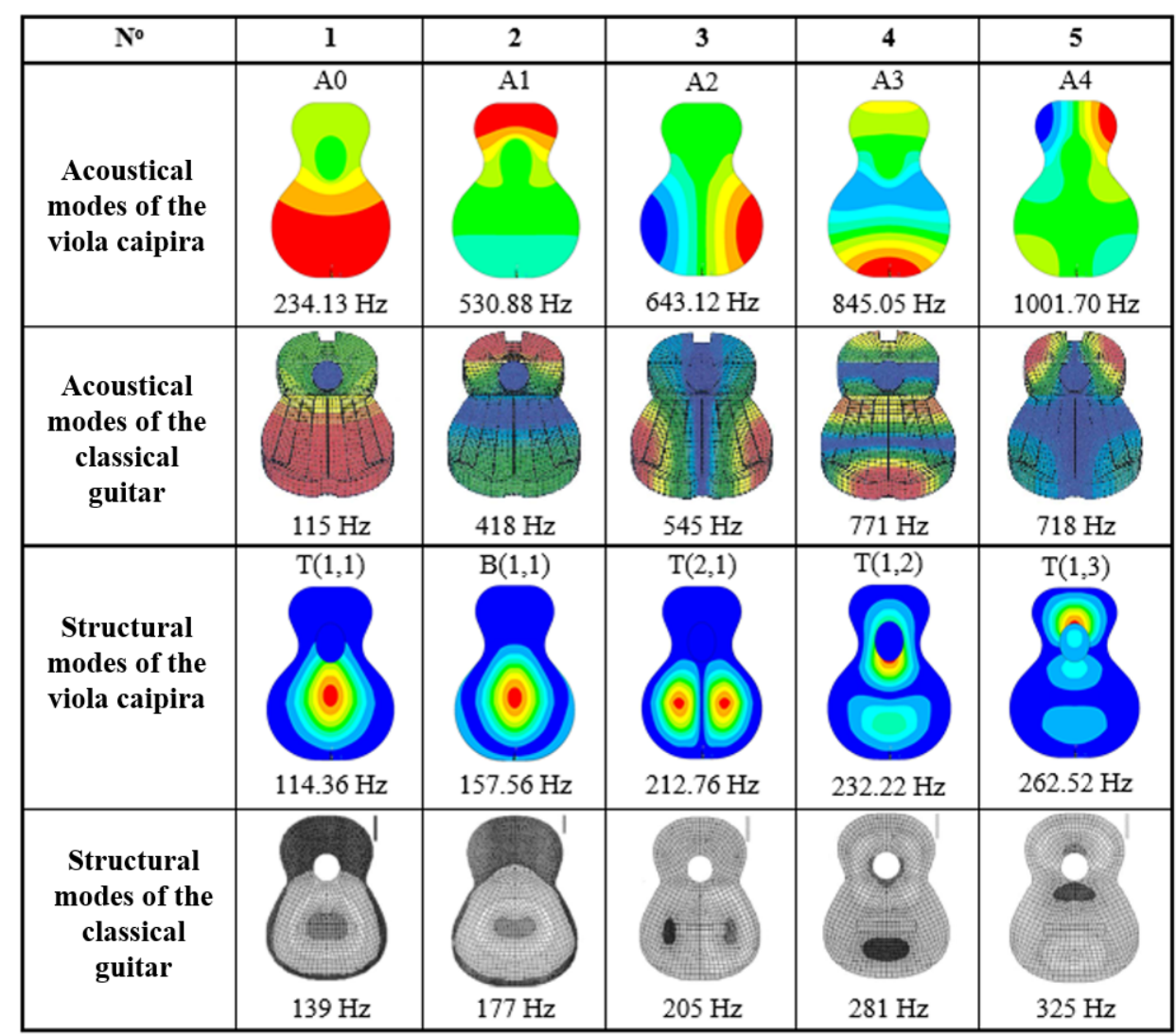

Figure 2.8: Comparison of the five first structural and acoustical numerical modes of the classical guitar and viola caipira resonance boxes (adapted from [?]). The classical guitar acoustical modes, shown in the second row, and structural modes, shown in the fourth row, were extracted from [?] and [?], respectively.

\subsection{Summary}

A bibliography review has been presented, where different pertinent aspects were approached:

- Description of the motion of ideal and stiff strings uncoupled from the body;

- String/body coupling methods and effects of such coupling on the string motion;

- Experimental methods used to characterize vibration and radiation of guitars;

- Finite difference schemes used for the sound synthesis of musical instruments;

- Previous works on the viola caipira. 



\section{Chapter 3}

\section{EXPERIMENTAL STUDY OF THE viola caipira}

The viola caipira has a particular timbre. The objective of this chapter is to identify experimentally sound and vibrational specificities of the instrument. Experimental tests are performed on a single instrument (Rozini brand, Ponteio Profissional model) tuned in Rio Abaixo (see Table ??). Although the viola caipira exists in a variety of types, the instrument studied in this chapter is representative since it has five double courses of strings, which is the most common configuration, and a very typical resonance box shape. This experimental study is divided in the following parts:

- Analysis of the string motion using a high speed camera;

- Analysis of a set of sounds resulting from instrument plucks;

- Vibrational analysis of the instrument body.

\subsection{Analysis of the string motion using a high speed camera}

\section{Experimental setup}

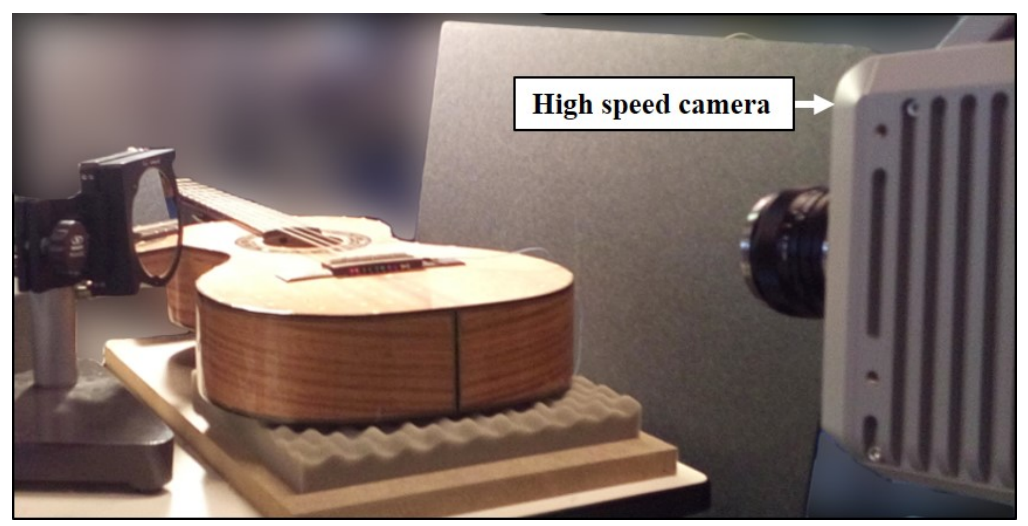

Figure 3.1: Experimental setup for motion analysis of the viola caipira strings using a high speed camera.

In order to analyse the motion of strings during and after a typical pluck, an experimental study was carried out using a high speed camera Photron, model FASTCAM SA-X2, which provided an imaging 
performance of $1024 \times 768$ pixels of resolution at the recording rate of 5000 frames per second. To avoid interference on the soundboard vibration and facilitate the observation of the strings motions, the guitar was placed horizontally on a flat surface with its back plate in contact with a piece of foam, as shown in Figure ??. A mirror attached to a stand was also positioned next to the instrument to capture images from a different angle than that captured by the camera.

\section{Double pluck}
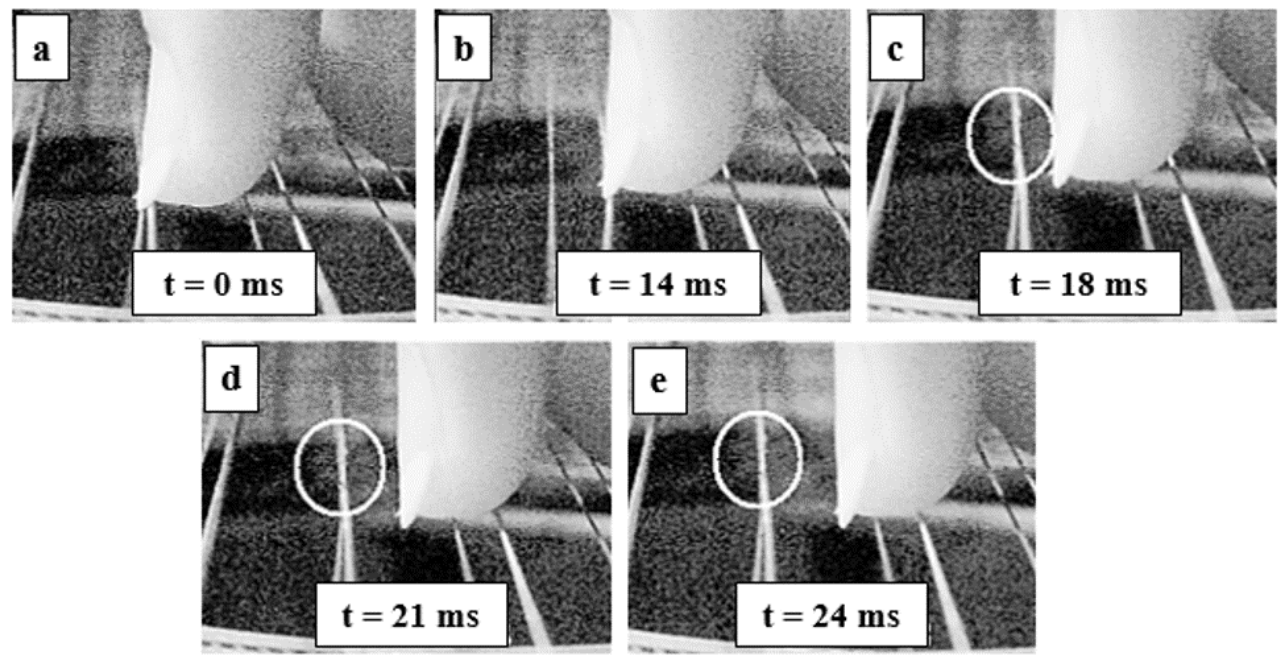

Figure 3.2: Series of snapshots captured during a viola caipira pluck with the thumbnail. (a) First pluck at string 8 (D4). (b) Second pluck at string 7 (D3) after 14 ms. Three successive collisions between the two strings are shown in (c), (d) and (e).

The fourth pair of strings (strings 7 and 8 ) was plucked in the downward direction of strings using the thumbnail, which is the usual way to pluck the pairs 3,4 and 5. As shown in the series of snapshots in Figures ??a and ??b, the excitation of the string pair is characterized by a double pluck: strings 8 and 7 are plucked successively and rapidly, within a time interval $\Delta t_{\text {pluck }}=14 \mathrm{~ms}$ for the presented measure. The objective of this analysis was not to measure precisely the time interval between the two successive plucks, but rather to understand how the string excitation occurs in the viola caipira since the strings are plucked by pairs, differently from classical guitars. A single image record is therefore sufficient to understand the excitation phenomena. It is worth mentioning, however, that the value of $\Delta t_{\text {pluck }}$ may be affected directly by factors such as spacing between strings, string diameters, instrument tuning and plucking direction. In addition, obtaining a $\Delta t_{\text {pluck }}$ value with reasonable approximation in terms of magnitude order will allow us to initialize in a more realistic way the algorithm for sound synthesis presented in Chapter 4.

\section{Collisions between strings}

Figures ??c to ??e show that the strings collided successively three times after the second pluck, within an interval of $3 \mathrm{~ms}$. This phenomenon of string/string collisions is a remarkable specificity of the vibrational behaviour of strings in the viola caipira and undoubtedly has a strong influence on the instrument sound. This issue will be addressed more specifically in Chapter 4 by means of physics-based sound syntheses.

Not shown here, many other plucks have been recorded. Different plucking conditions were used and 
different strings were excited. It was found qualitatively that the occurrence of string/string collisions varies from pair to pair and depends on the string spacing, excitation force and plucking direction. Collisions were observed only in cases where pairs 3,4 and 5 were plucked in a direction predominantly parallel to the soundboard by applying moderate force. Since the tensions on the strings of pairs 1 and 2 are relative higher, the amplitude of vibration of those strings is smaller considering that the same excitation force is applied. Since the tensions on the strings of pairs 1 and 2 are relative higher, their vibration amplitudes are in general smaller considering that the same excitation force is applied. Thus, to induce collisions in pairs 1 and 2, a higher plucking force is required. Even though in a qualitative way, such observations give us reasonable results to conclude that the existence of string/string collisions is associated to characteristics intrinsic to the instrument (string tensions), parameters controlled by the musician (direction and force of the pluck) and and parameters adjusted by the instrument maker (adjustment of string spacing).

It is worth suggesting that string/string collisions may also occur in plucked instruments where strings are arranged and played by pairs such as mandolins, twelve-string guitars and Portuguese guitars. To the knowledge of the author, such phenomenon is not reported in the literature yet.

\subsection{Sound analysis}

The experimental work presented in this section consisted in recording the sound resulting from two configurations of the instrument: first a single string was excited while all the others were completely damped so they were prevented from vibrating by sympathy; the same string was then excited under the same conditions, but all the others strings were left free to vibrate. Time-frequency and energy analyses of the recorded sounds were obtained.

\section{Experimental setup}

The experiment was performed in the laboratory environment where noise interference from secondary sources was minimized. Measurements were performed using the following protocol. The viola caipira is supported in a fixed position using a guitar stand. The instrument is hung by the head and its body is fixed with modelling clay at the two contact points between its back plate and the stand feet. PCB microphone is positioned $12 \mathrm{~cm}$ from the soundboard pointing towards the sound hole. A $56 \mu \mathrm{m}$ copper wire is used to excite the string. The wire is placed around the string 1 (D4, 293.67 $\mathrm{Hz}$ ), at $8 \mathrm{~cm}$ from the bridge, and then is pulled aside in the direction normal to the soundboard until it breaks abruptly. The wire excitation allows controllable and repeatable plucks since its breaking force is expected to be quasi-invariable (for more details on the wire technique, the reader is invited to refer to the article presented in the end of this chapter). When necessary, strings are completely damped by using foam and cloth without contact with the soundboard. Signal acquisition is performed using National Instrument I/O interface running at $44.1 \mathrm{kHz}$.

\section{Double decay rate, string sympathetic resonances and beating}

The analysis of the sound decay is done by means of the so-called energy decay curve (EDC), which is computed by the backward integration [?]:

$$
E D C(t)=\int_{t}^{T} s^{2}(\tau) d \tau,
$$


where $\mathrm{s}(\mathrm{t})$ is the analysed signal and $\mathrm{T}$ its total length.

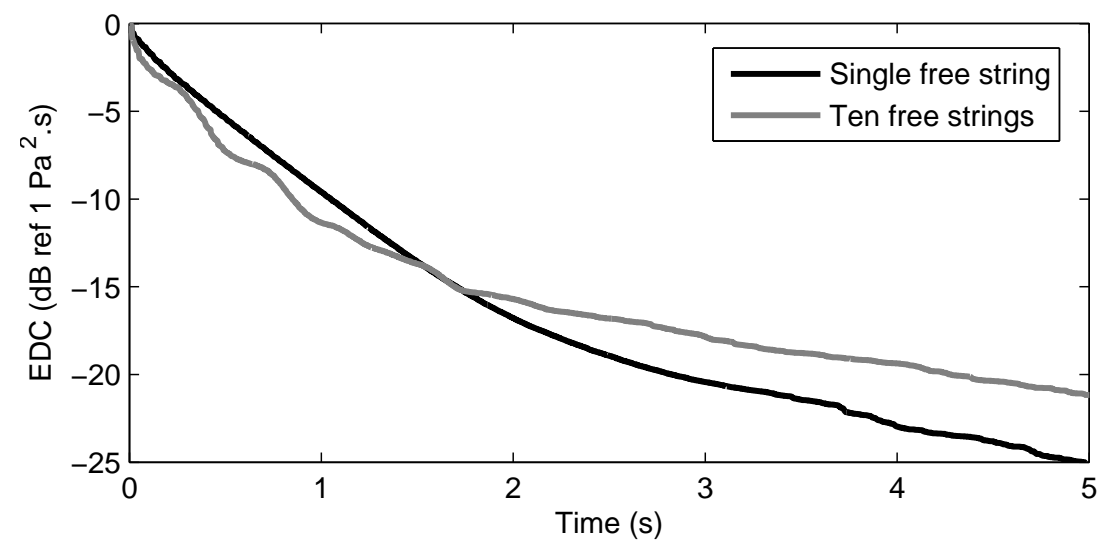

Figure 3.3: Sound energy decay curves obtained when the string 1 (D4, 293.67 Hz) is plucked by means of a copper wire in the direction normal to the soundboard. Two configurations are used: all the other strings are completely damped (black curve) or left free to vibrate (gray curve).

The complexity of the energy decay is explained mainly by the coupling between polarizations of a given string and by the coupling between different strings through the bridge motion. Figures ?? and ?? illustrate the effects of these different couplings when the string 1 (D4, 293.67 Hz) is plucked. Three effects are identified as follows.

\section{Double decay rate}

Two phases can be defined during the sound energy decay:

- Attack phase, associated to the immediate sound during which the decay is faster. In this phase, the decay is mainly explained by the coupling of the string with the flexural motion (out-of-plane motion) of the soundboard. This coupling is relative strong and gives rise to a pumping energy mechanism by the soundboard. For the string, this corresponds to a mechanism of damping.

- Sustain phase, associated to the aftersound during which the decay is slower. In this phase, the decay is explained by the coupling of the string with the soundboard in-plane motion.

This mechanism of double decay rate is clearly visible in Figure ??, which shows the sound energy decay curves obtained when the string $1(\mathrm{D} 4,293.67 \mathrm{~Hz})$ is plucked with all the other strings being completely damped (black curve) or free to vibrate (gray curve). The fact of isolating the string limits the couplings at the origin of the decay so that the aftersound is simplified.

\section{String sympathetic resonances}

Figure ?? shows a higher aftersound level when the 10 strings of the instrument are free. Couplings between strings through the bridge lead to string sympathetic resonances, which add energy to the aftersound that would come from the isolated string. This aftersound augmentation due to the strings coupling is perceived as a "halo of sound" [?], which is a characteristic of the instrument. 


\section{Beating}

The string modes associated to the two polarizations are slightly perturbed by the bridge motion so that they have very close frequencies [?]. These small frequency differences between string components can lead to beating tones, which are perceived with periodic variations in volume. This phenomenon may appear on one or more partials of the same string, which is visible on the spectrogram of Figure ??, or even on a more complex interaction between partials of two or more strings as shown in the spectrogram of Figure ??.

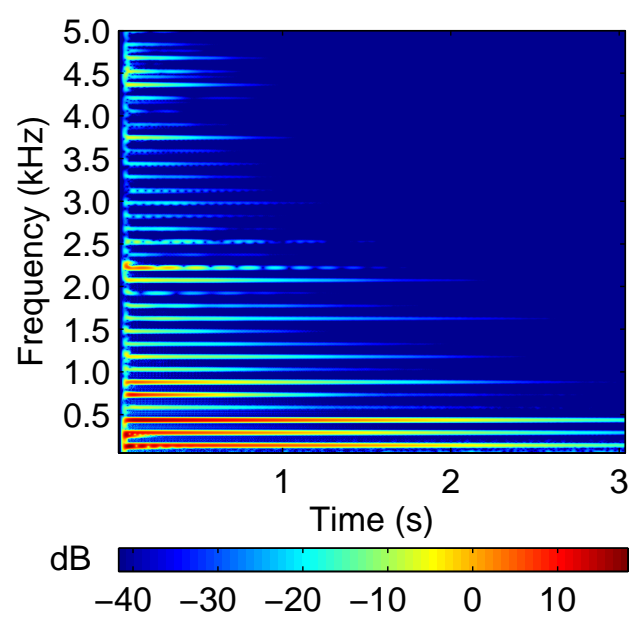

(a) Single free string.

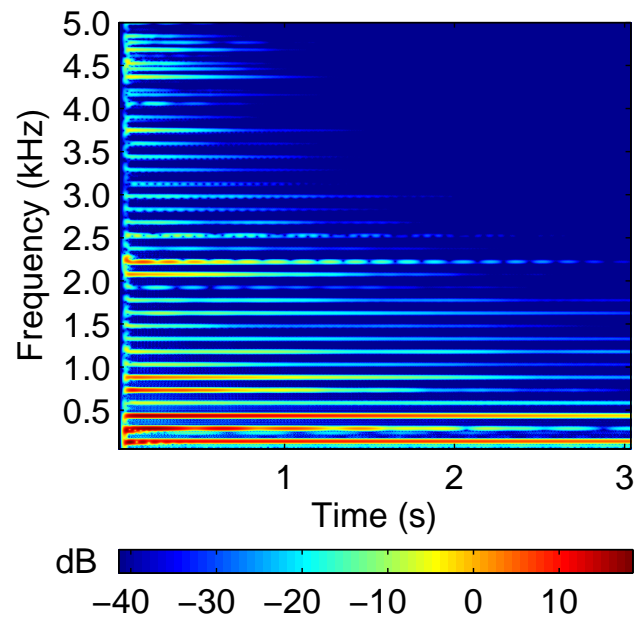

(b) Ten free strings.

Figure 3.4: Sound spectrograms obtained when the string 1 (D4, 293.67 Hz) is plucked in the direction normal to the soundboard using two different configurations: (a) all the other strings are completely damped (associated to the black energy decay curve in Figure ??); (b) all the other strings are left free to vibrate (associated to the gray energy decay curve in Figure ??).

\subsection{Vibrational analysis of the body}

After identifying in the previous section some specificities of the sound of the viola caipira, searching for vibrational specificities of the instrument is the aim of this section. Do the vibrational modes of the viola caipira have particularities which differ the instrument from the classical guitar or other guitars?

\subsubsection{Modal analysis of the soundboard}

In order to investigate the dynamical behaviour of the viola caipira soundboard, which is assumed a linear mechanical system, an experimental modal analysis was performed to obtain natural frequencies, modal damping factors and respective mode shapes.

\section{Experimental setup}

The roving hammer technique was used to excite the instrument at 118 points along the soundboard using a miniature impact hammer to provide a broad-band excitation (PCB 086E80, sensitivity 20.06 $\mathrm{mV} . \mathrm{N})$. Acceleration responses resulting from the hammer excitations were collected by a lightweight 


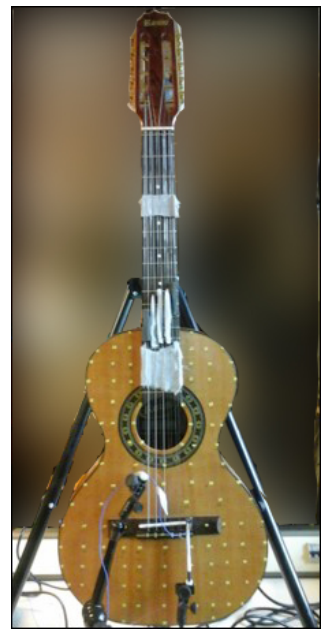

(a)

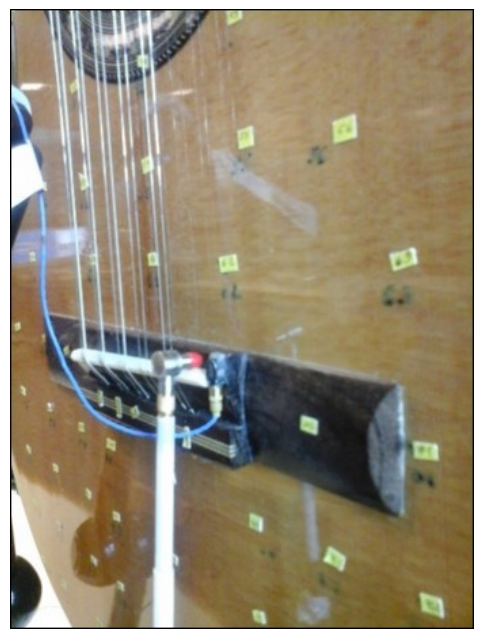

(b)

Figure 3.5: Setup used for the experimental modal analysis of the viola caipira soundboard.

accelerometer with a mass of $2 \mathrm{~g}$ (PCB 352C23, sensitivity $0.523 \mathrm{mV} /\left(\mathrm{m} . \mathrm{s}^{2}\right)$ ) fixed on the bridge and mounted on a thin wax layer, as shown in Figure ??b. LMS Scadas was used to record the acceleration and force data for further analysis. A guitar stand was used to hang the instrument by the head, as shown in Figure ??a. The strings were completely damped with a light cloth so that their tensions on the body instrument were kept.

\section{Typical result for the inertance transfer function}

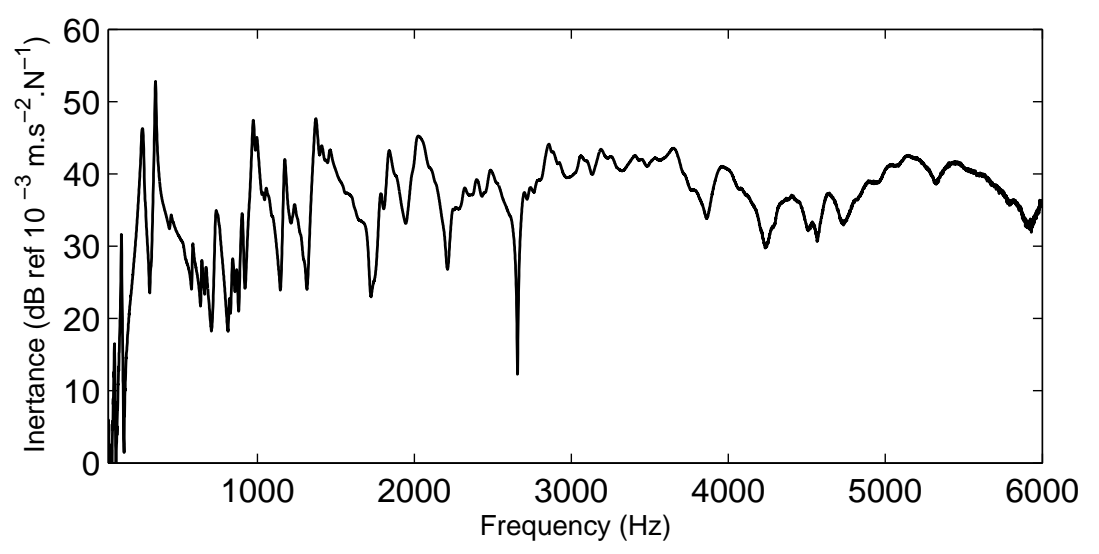

Figure 3.6: Modulus of a typical inertance transfer function measured at the bridge of the viola caipira. Excitation and response points are shown in Figure ??

The inertance transfer functions were calculated between all excitation points and the fixed acceleration response point. Figure ?? shows typical variations of the modulus of the inertance measured 
at the bridge of the viola caipira. This plot have numerous modal contributions leading to a complicated pattern. At low frequencies (below about $1500 \mathrm{~Hz}$ ) the system is characterized by relatively isolated modes that can be easily extracted using classical Fourier based modal identification techniques. In the mid and high-frequency ranges, the modal overlap is too large to allow robust modal identification since modal contributions are no longer individually observable.

\section{Mobility transfer function and mean mobility}

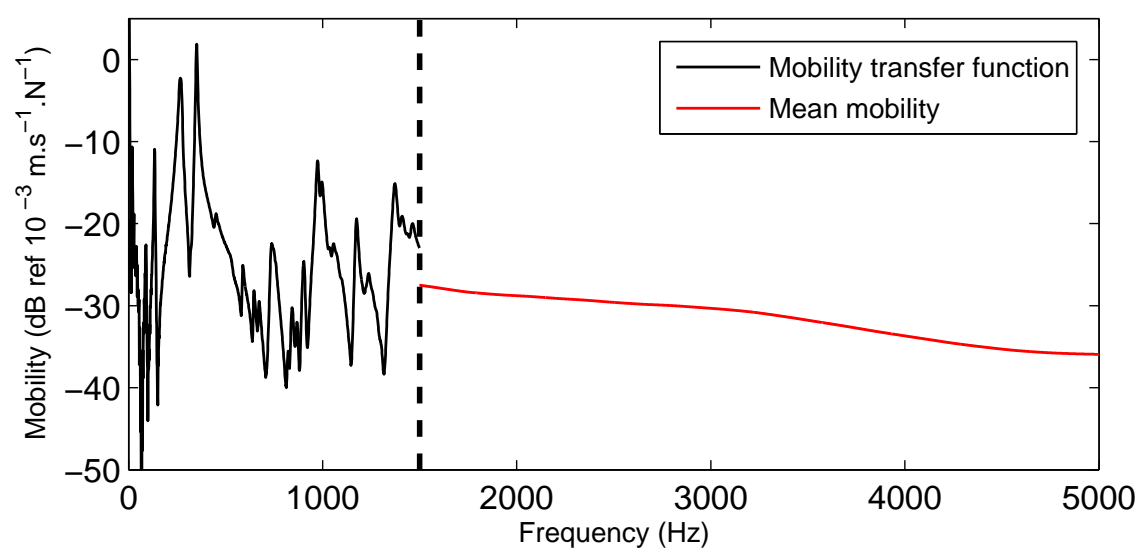

Figure 3.7: Overall representation of the mobility measured at the bridge of the viola caipira

By integrating the inertance curve shown in Figure ??, one may obtain its associated mobility transfer function. Figure ?? shows an overall description of such mobility. The so-called mean mobility (also called characteristic admittance [?]) is calculated from $1500 \mathrm{~Hz}$. It consists in computing the mean value of the mobility, in $\mathrm{dB}$, included in a sliding window of a certain span, this latter moving from a sample to the next. Thus, the mean mobility $G_{C_{d B}}$ writes

$$
G_{C_{d B}}\left(f_{c}\right)=\frac{1}{\Delta f} \int_{f_{1}}^{f_{2}} Y_{d B} d f,
$$

where $\Delta f=f_{2}-f_{1}, f_{c}=\frac{f_{1}+f_{2}}{2}, f_{1}$ and $f_{2}$ being respectively the lower and upper frequency bounds of the sliding window. This quantity has been found useful to characterize and compare stringed instruments $[?, ?]$ since it allows a description of vibratory responses in the mid and high-frequency ranges, where the modal overlap is too large. The viola caipira soundboard, like classical guitar soundboards have been shown to plate-like systems: their mean mobilities are nearly independent on the frequency. This property is the one of a plate, whose equivalent parameters can be computed (cf. [?]).

\section{Modal identification}

To extract the modal parameters from the 118 obtained inertance transfer functions, a modal identification procedure using the PolyMAX method [?] implemented into LMS Test.lab software was carried and 36 modes were extracted between 0 and $1500 \mathrm{~Hz}$, leading to the synthesized inertance curve shown in Figure ??. 
Figure ?? shows the natural frequencies and respective damping ratios of the 36 soundboard modes identified between 0 and $1500 \mathrm{~Hz}$. The values of damping ratios are of the order of few percent $(0.15-$ $2.1 \%$ ), which is a usual range in the context of guitar-like instruments [?].

Figure ?? shows that the four first modes of the viola caipira soundboard resemble those of the classical guitar (see for example [?] for a thorough description) with frequencies moderately higher since the resonance box of the measured instrument is smaller. In this work mode shapes are labelled according to the convention used in [?]. $T(1,1)_{1}$ mode, also denoted $A_{0}$, is characterized by the motion of the lower bout of the soundboard and mainly by a significant acoustic motion of the air piston in the sound hole, which is not measured here. The $T(1,1)_{2}$ mode arises due to the coupling of the soundboard with the air cavity and is often referred to the "fundamental soundboard mode". $T(1,1)_{1}$ and $T(1,1)_{2}$ modes have similar shapes. Higher order modes such as $\mathrm{T}(2,1)$ and $\mathrm{T}(3,1)$ have nodal lines separating vibrational lobes.

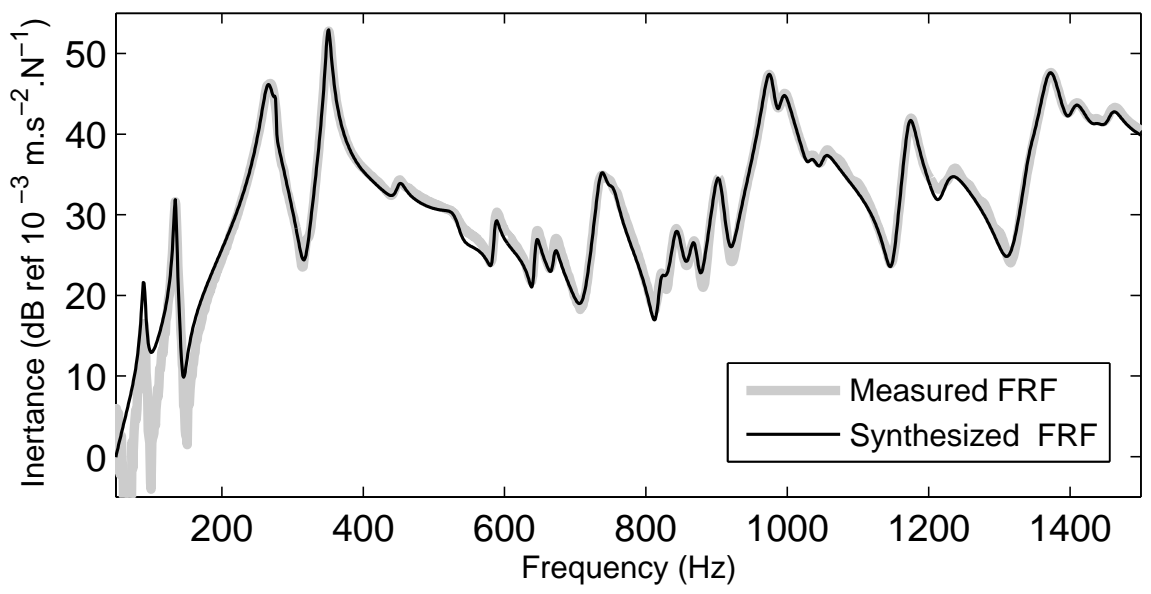

Figure 3.8: Measured (gray line) and synthesized (black line) inertance transfer functions of the viola caipira. Corresponding excitation and response points are shown in Figure ??b. 


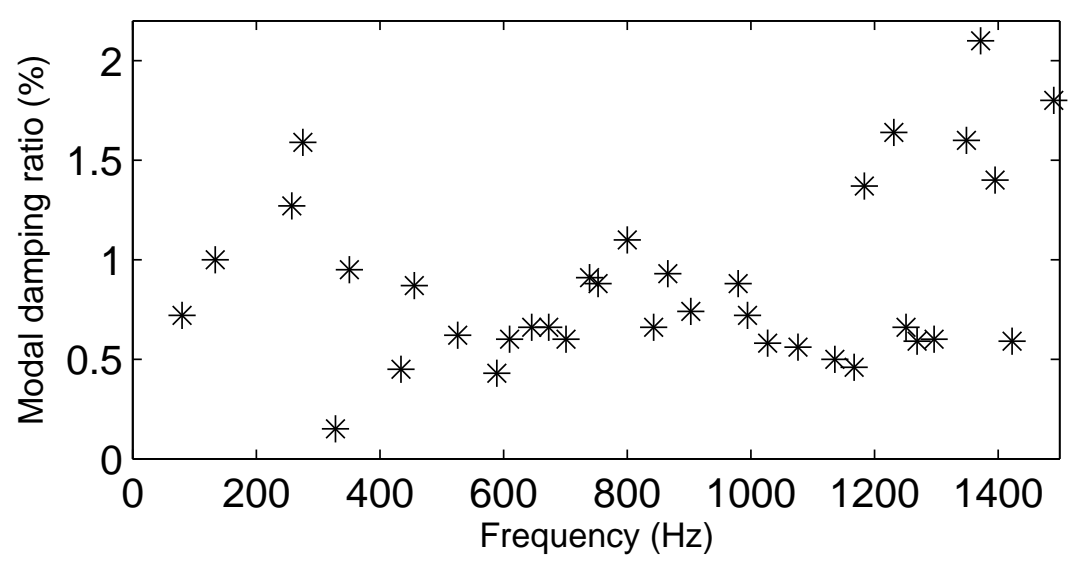

Figure 3.9: 36 natural frequencies and respective damping ratios obtained from the modal analysis of theviola caipira soundboard.

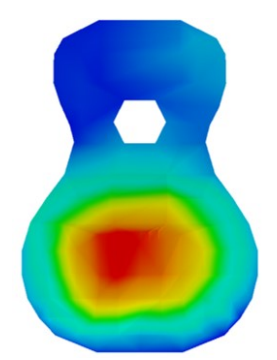

(a) $T(1,1)_{1}, 133.8 \mathrm{~Hz}$

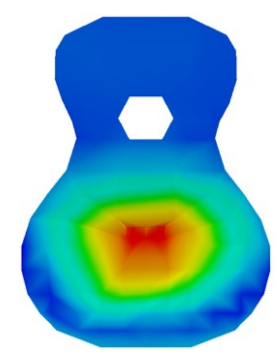

(b) $T(1,1)_{2}, 261.7 \mathrm{~Hz}$

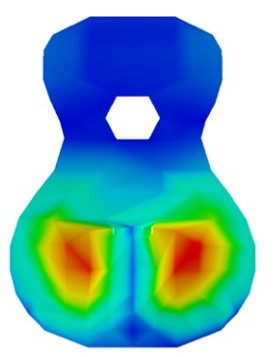

(c) $\mathrm{T}(2,1), 351.8 \mathrm{~Hz}$

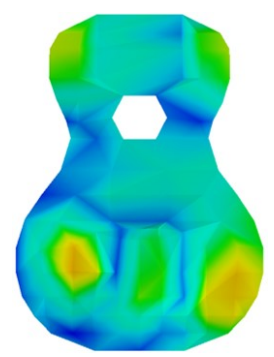

(d) $\mathrm{T}(3,1), 752.9 \mathrm{~Hz}$

Figure 3.10: Examples of mode shapes and respective natural frequencies obtained for the viola caipira soundboard. Mode shapes are labelled according to the convention used in [?].

\subsubsection{Operating deflection shape analysis}

The standard method for determining the modes of a structure consists of measuring a large number (usually several tens) of transfer functions and performing a modal fit as has been done in the previous paragraph. An alternative to this technique is to use a scanning laser vibrometer. This tool automatically allows to collect a very large number (several thousand) of transfer functions and, without performing a modal fit, one can observe the vibrational levels at a given frequency. In this case, therefore, the obtained of the vibration pattern of the structure is an operating deflection shape (ODS). When the modal overlap is small, an operating deflection shape at a given resonance peak can be interpreted as a mode shape.

\section{Experimental setup}

In this experiment, optical measurements allowing non-contact data acquisition were performed to analyse the vibrational behaviour and obtain the ODSs of the front of the viola caipira. A vibration mapping of the instrument soundboard and neck was obtained by using a Polytech PSV-500 laser scanning vibrometer and an automatic hammer Maul-Theet vImpact-60, as shown in Figure ??. As in the experimental modal analysis of the soundboard, the instrument was suspended using a guitar stand and 


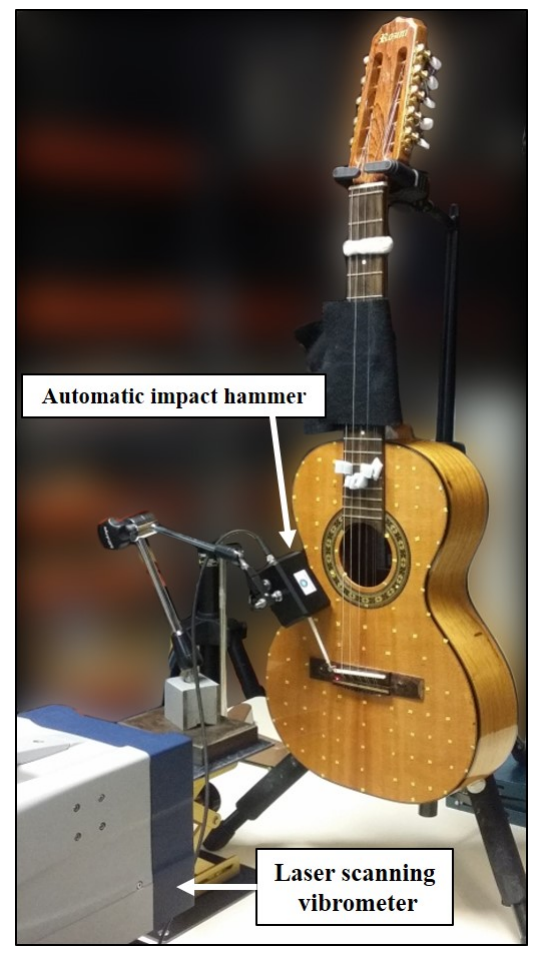

Figure 3.11: Setup composed by an automatic impact hammer and a laser scanning vibrometer used for measuring the vibration response on the front of the viola caipira. 


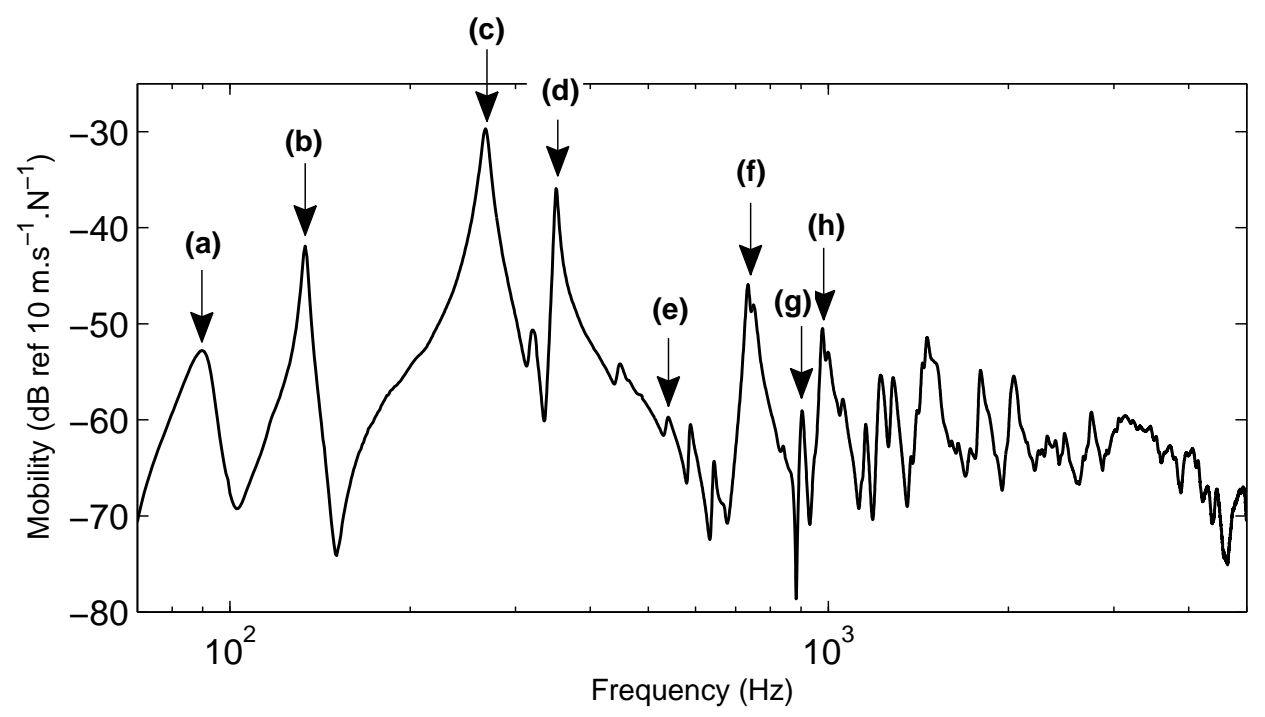

Figure 3.12: Typical mobility transfer function measured at the viola caipira bridge using laser scanning vibrometer.

strings were completed damped. Impulse excitations over a broad frequency band were provided by the automatic hammer at a fixed position at the bridge. A set of time-domain response signals at 1000 points was measured normal to the soundboard using the scanning vibrometer, which was positioned about 1.5 $\mathrm{m}$ distant from the instrument. By means of the PSV software linked to the vibrometer, an FFT analysis was performed and mobility curves up to $5000 \mathrm{~Hz}$ were obtained for the multiple measurements.

\section{Typical results for ODSs}

ODSs b, c, d and $\mathrm{f}$ in Figure ?? correspond respectively to modes $T(1,1)_{1}, T(1,1)_{2}, \mathrm{~T}(2,1), \mathrm{T}(3,1)$ in Figure ??, which allows to confirm that the ODS analysis gives results close to those obtained with the classical modal analysis. Some minor differences are however visible mainly between corresponding frequencies. Small variations in the instrument tuning, hygrometry of the enviroment, instrument boundary conditions and the perturbation due to the mass of the accelerometer used in the modal analysis may explain such differences.

\subsubsection{Mobility variation along the bridge saddle}

The most of string energy vibration converted into radiated sound is transferred to the body through the bridge at the coupling points along the saddle. This energy exchange can be studied by mobility measurements at the bridge, which quantify the conversion of string force into bridge velocity. Mobilities at the 10 coupling points are measured in this subsection . In practice, measurements were performed at the vicinity of the string/saddle contact points, as shown in Figure ??, because the obtained signal-to-noise ratio was much better. 


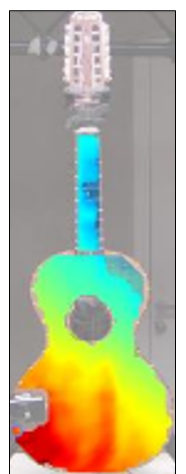

(a) $95.3 \mathrm{~Hz}$

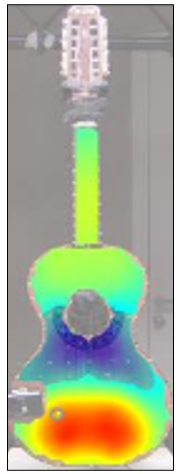

(e) $T(1,2), 542.2 \mathrm{~Hz}$

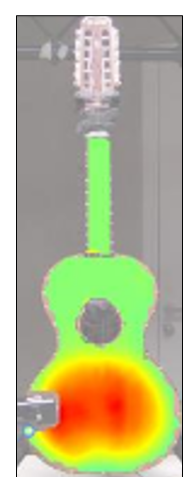

(b) $T(1,1)_{1}, 129.7 \mathrm{~Hz}$

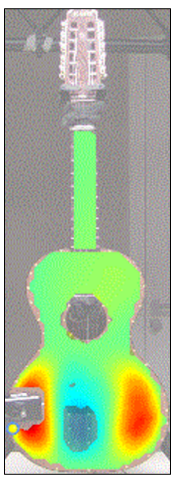

(f) $T(3,1), 751.6 \mathrm{~Hz}$

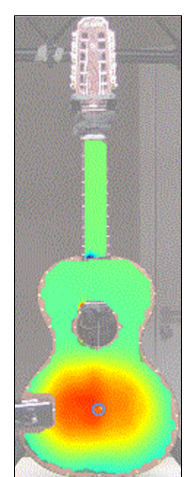

(c) $T(1,1)_{2}, 264.1 \mathrm{~Hz}$

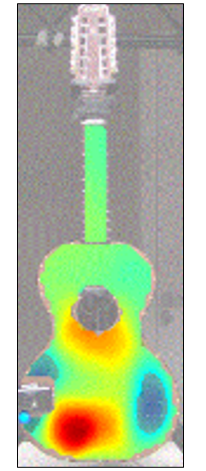

(g) $T(2,2), 908.6 \mathrm{~Hz}$

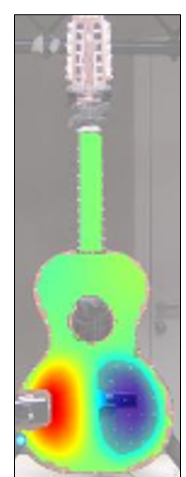

(d) $T(2,1) .356 .3 \mathrm{~Hz}$

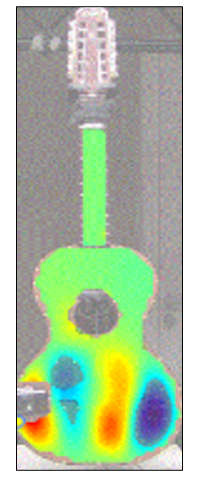

(h) $T(4,1), 984.4 \mathrm{~Hz}$

Figure 3.13: Eight examples of operating deflection shapes of the front of the viola caipira and respective natural frequencies. 


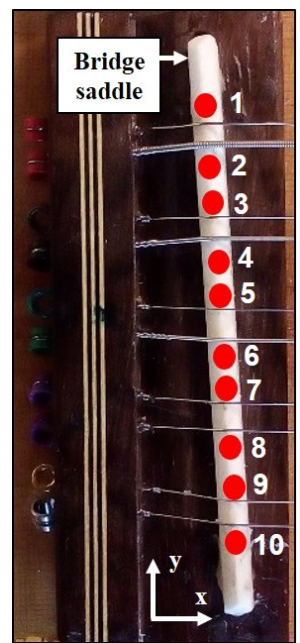

(a)

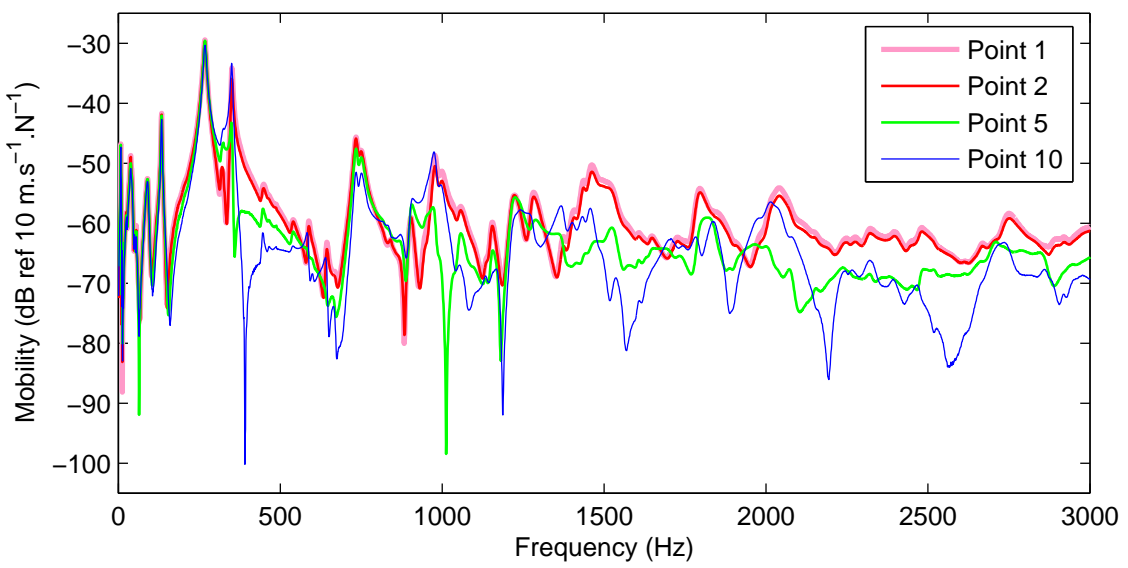

(b)

Figure 3.14: (a) Mobility measurement positions 1 to 10 on the bridge saddle of the viola caipira; (b) Mobilities measured at points 1, 2, 5 and 10 .

When the points are close, the mobilities are similar as shown in Figure ?? (see mobility curves at points 1 and 2). The differences between some mobilities are explained by the fact that mode shapes vary along the saddle. In particular, some mode shapes have nodal regions at the saddle and may not contribute to certain mobilities (see for example mode shape $\mathrm{T}(2,1)$ at point 5 ).

Figure ?? summarizes the set of mobilities measured along the saddle as a function of the string attachment points and frequency. In this figure the values of fundamental frequencies of certain notes are added, which gives information about the energy exchange between strings and body. At a given frequency, the higher the mobility is, more quickly the energy is radiated by the body so that the sound produced is relatively powerful with a short duration. According to Figure ?? this may occur, for example, when the notes $\mathrm{C} 4$ and $\mathrm{C} 4$ are played in strings 3, 4, 5 and 10. Conversely, smaller mobility leads to a slower energy flow resulting in a less powerful sound with a longer duration, which may occur when G4 is played in string 4 , for example. 


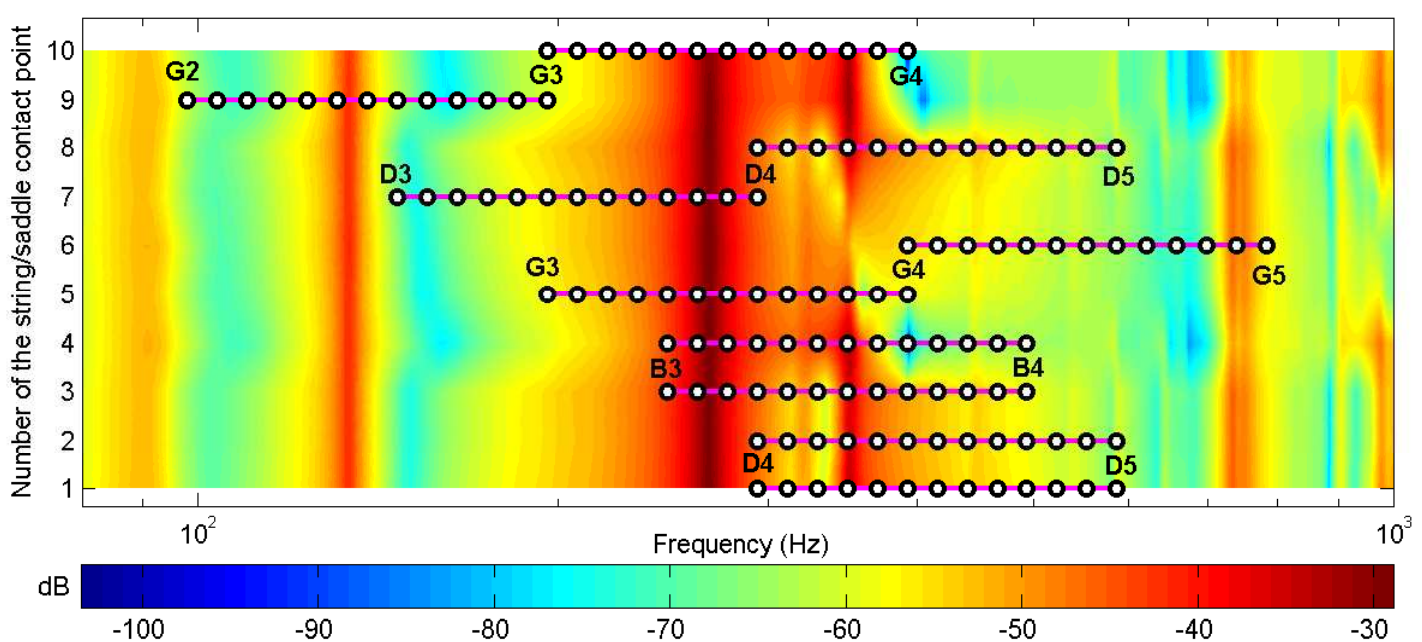

Figure 3.15: Mapping of mobility along the bridge saddle obtained from the interpolation of mobilities measured at the 10 points shown in Figure ??. Mobilities are obtained in the frequency range between 80 $\mathrm{Hz}$ and $1000 \mathrm{~Hz}$ on which fundamental frequencies of notes from frets 0 to 12 are indicated for each string considering the Rio Abaixo tuning type.

\subsubsection{Cross term of the bridge inertance matrix: coupling between horizontal and vertical motions}

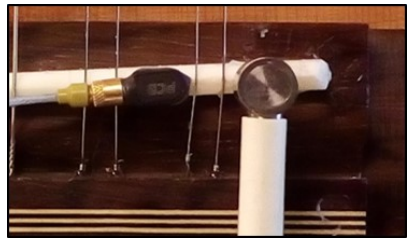

(a)

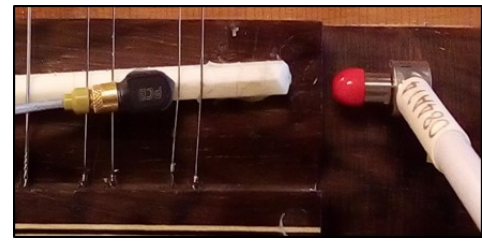

(b)

Figure 3.16: Setup for measuring direct (a) and cross (b) inertance transfer functions at the bridge of the viola caipira.

Forces and acceleration responses at the bridge have in-plane and out-of-plane components. The 2Dinertance matrix is a quantity describing the coupling between these components. The cross term of the 2D-inertance matrix expresses the coupling between horizontal and vertical motions in the bridge. Cross and direct terms, defined respectively by $H_{z y}(\omega)=a_{z}(\omega) / F_{y}(\omega)$ and $H_{z z}(\omega)=a_{z}(\omega) / F_{z}(\omega)$, are measured in the directions shown in Figure ??. The variations of the inertances with the frequency are shown in Figure ??. It is clear that the cross term of the inertance matrix is lower than the direct term, which shows that the coupling between polarization induced by the motion of the bridge exists but is moderate. 


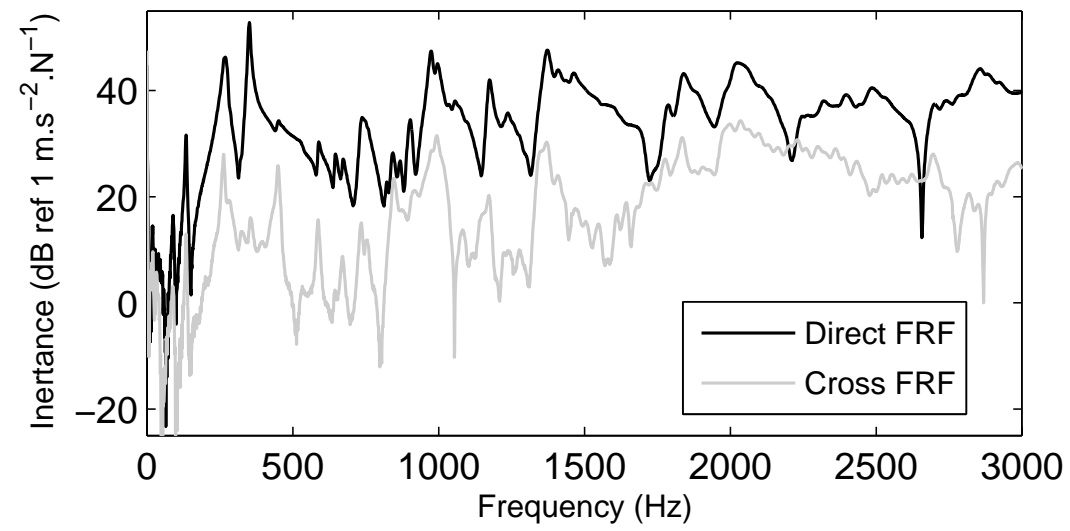

Figure 3.17: Typical results for cross and direct inertance transfer functions measured at the bridge of the viola caipira.

\subsubsection{Mobility measurements and modal analysis at the bridge of a viola caipira using the Roving Wire-Breaking Technique}

This subsection is presented as an article entitled "The Roving Wire-Breaking Technique: a low cost mobility measurement procedure for string musical instruments", submitted to the Applied Acoustics Journal in October 2017. In that article, the mode shape components at the bridge of a viola caipira are estimated using a high resolution modal analysis of mobility measurements obtained by means of a novel technique named "Roving Wire-Breaking Technique". 


\title{
The Roving Wire-Breaking Technique: a low cost mobility measurement procedure for string musical instruments
}

\author{
${ }^{1,2}$ G. O. Paiva, ${ }^{1}$ F. Ablitzer, ${ }^{1}$ F. Gautier and ${ }^{2}$ J. M. C. dos Santos \\ ${ }^{1}$ Université du Maine, LAUM, Avenue Olivier Messiaen, 72085 Le Mans cedex 9, France \\ ${ }^{2}$ University of Campinas, UNICAMP-FEM-DMC, Rua Mendeleyev, 200, CEP 13083-970 Campinas, SP, Brazil
}

\begin{abstract}
Bridge mobilities are usually used to characterize the couplings between the strings and the body of plucked or bowed string instruments. Such transfer functions are classically measured using impact hammer technique. An alternative method called wire-breaking method (also known as step relaxation method), introduced initially for the excitation of large structures is investigated in this paper. The method has been recently adapted to string instruments: it consists in placing a thin copper wire around the string in a position very close to the bridge saddle and pulling aside in the direction of interest until the wire breaks abruptly imparting a step function force to the driving point. When carried out with damped strings, the acceleration of the bridge measured with a miniature sensor provides a good estimation of transfer mobilities. The limits of the technique in terms of repeatability and signal-tonoise ratio are investigated making use of comparisons with results obtained by the classical impact hammer method. It is finally shown that the bridge admittances measured using the "Roving WireBreaking Technique" may be used to identify mode shapes components at the bridge using a high resolution modal analysis. Since no force sensor is needed to measure mobility, the technique is low cost and can be used in the instrument maker workshop for instrument modal characterization.
\end{abstract}




\subsection{Introduction}

The sound produced by string musical instruments is the result of interactions of several subsystems: the excitation mechanism, the strings, the instrument body, the air and the listener. In the acoustical guitars and violins, the most of the energy that will be converted into radiated sound is transferred to the body through the bridge. The energy flow from each string depends primarily on how strongly it is coupled to the body: the stronger the coupling, the quicker the energy is transferred to the body. The bridge mobility (or admittance), defined as the ratio in the frequency domain between velocity and force, is an indicator of the string/body coupling. For plucked string instruments the mobility governs the compromise between duration and power. For bowed string instruments, the mobility is one of the key factors determining playability. When considering velocity and force in the out-of-plane and in-plane directions, the mobility takes the form of a 2-D matrix whose diagonal terms describe the degree of coupling with the body in each direction considered separately and whose cross terms describe the coupling between the two string polarizations.

Mobility measurements are typically made to characterize and compare string instruments [?, ?, ?, ?, ?, ?, ?, ?]. Mobilities measured at the bridge of string instruments can be also used to feed several sound synthesis models for plucked [?, ?] and bowed [?, ?] string instruments based on the modal description of strings and body separately. In such hybrid methods, modal parameters of the body are obtained from experimental data while string modal parameters are defined from analytical models.

This work is directly linked to the development of a tool for instrument makers (PAFI, Plateforme d'aide à la facture instrumentale, available at http://pafi.univ-lemans.fr/) which aims at supporting the maker decisions when building or adjusting musical instruments. This tool consists of online postprocessing packages, including hybrid sound synthesis, a low cost bridge mobility measurement system and a musical instrument database [?, ?, ?, ?]. As a consequence, there is a need to develop a low cost methodology for measuring body modal parameters (frequencies, damping coefficients and mode shapes components at the coupling points).

The main goal of this paper is to investigate the capability of the wire breaking technique to play this role. Such technique is not widely used and consists in exciting the instrument body by placing a thin copper around a string very close to the bridge and pulling it until it breaks. The limitations of this low cost technique in terms of signal-to-noise ratio and repeatability are pointed out. The paper is organized as follows: in the rest of the current section a bibliography review on the wire excitation technique is presented, followed by the statement of the problem of using the classical hammer method for measuring mobilities at the bridge of string instruments. Section ?? presents the principle of the wire-breaking method and the experimental setup used for the measurements presented in this paper. In Section ??, the limitations of the wire-breaking method are investigated by making comparisons with results obtained by the hammer method. A calibration method for mobility measurement is proposed in Section ??. Finally, in Section ??, the "Roving Wire-Breaking Technique" is used to identify mode shapes at the bridge from mobility measurements.

\subsubsection{Bibliography review}

The wire-breaking method is based on the analysis of the response of a structure to a step force. Also known in other fields as "step relaxation method", this method has been investigated in the dynamic characterization of some engineering systems such as wind turbines [?, ?], bridges [?, ?, ?] and launch vehicles [?], where mechanical excitations for modal testing are not so easy to produce (see Figure ??). In the musical acoustics context, such technique has been introduced by Woodhouse [?] in two different applications. Firstly, it was used for obtaining controlled pluck responses on classical guitars: the wire 
provides at the pluck position a repeatable excitation in terms of level of stress, in the direction of interest. The acoustic and vibration responses were recorded using a microphone and accelerometer, respectively, allowing comparisons with synthesized sounds. Secondly, the method was employed to measure mobility curves at the bridge of a classical guitar, which allowed to feed sound synthesis models. Calibrated mobilities were obtained by comparison with measurements obtained with impact hammer and vibrometer laser previously calibrated. In [?], a guitar sound synthesis has been obtained from a passive admittance modelling whose parameters were extracted from admittance curves measured at the bridge using the wire-breaking technique. In [?], mobility measurements on cellos using a copper wire were carried out with a pickup system mounted on the bridge to collect the input force signals at the string notches. The measurements were compared with hammer excitation and normal bowing: nothing fundamentally different was observed between those methods. The wire technique was also used in [?] for measuring the bridge impulse response on violins with completely damped strings: the string excited at the bowing position leads the breaking wire to impart an impulse that runs along the string and hits the bridge. In [?], the wire excitation allowed a controllable pluck at different string positions: the recorded sounds using a microphone were submitted to a high resolution modal analysis and the modal contributions of string and body on different guitar sounds were identified. Finally, in [?], a copper wire has been used to pluck a rigidly anchored string. Optical sensors were used to measure the resulting signals from which modal parameters were extracted via a high resolution method and used to feed the string damping model proposed in [?].

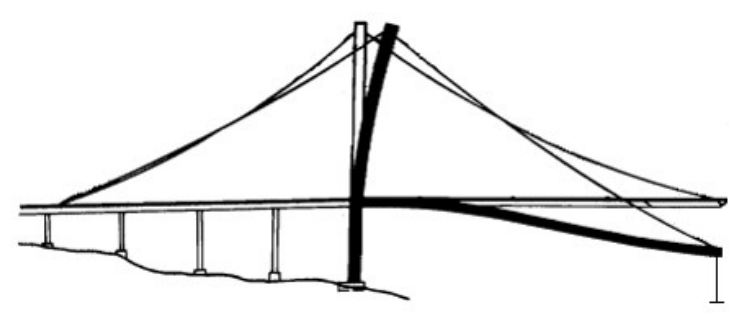

(a)

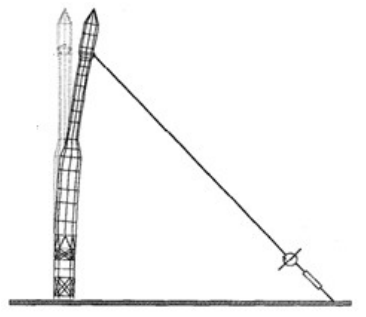

(b)

Figure 1.18: Schematic illustrating applications of the step relaxation method to excite (a) a bridge [?] and (b) a launch vehicle [?].

\subsubsection{Statement of the problem}

For a linear system, the mobility transfer function $Y_{i j}(\omega)$ is defined in the frequency domain as the ratio between the velocity response $V_{i}(\omega)$ at degree of freedom $i$ due to the force $F_{j}(\omega)$ applied at the degree of freedom $j$,

$$
Y_{i j}(\omega)=\frac{V_{i}(\omega)}{F_{j}(\omega)}
$$

where $\omega$ is the angular frequency.

For bowed and plucked string instruments, the mobility measured at the bridge quantifies the conversion of string force into bridge velocity. Both string forces and bridge velocities are assumed to be composed by two orthogonal components, parallel and perpendicular to the soundboard, corresponding 
respectively to $y$ and $z$ directions as shown in Figure ??, so that

$$
\left[\begin{array}{c}
V_{y}(\omega) \\
V_{z}(\omega)
\end{array}\right]=\mathbf{Y}\left[\begin{array}{c}
F_{y}(\omega) \\
F_{z}(\omega)
\end{array}\right],
$$

where $\mathbf{Y}$ is the $2 \times 2$ mobility matrix defined as

$$
\mathbf{Y}=\left[\begin{array}{cc}
Y_{y y}(\omega) & Y_{y z}(\omega) \\
Y_{z y}(\omega) & Y_{z z}(\omega)
\end{array}\right]
$$

The description above neglects both string and bridge longitudinal motions since the parallel and perpendicular components are much higher. It is also assumed that no torque is exerted on the body when forces are applied to the driving points, so that the component in the $x$ direction is ignored (cf. [?]).

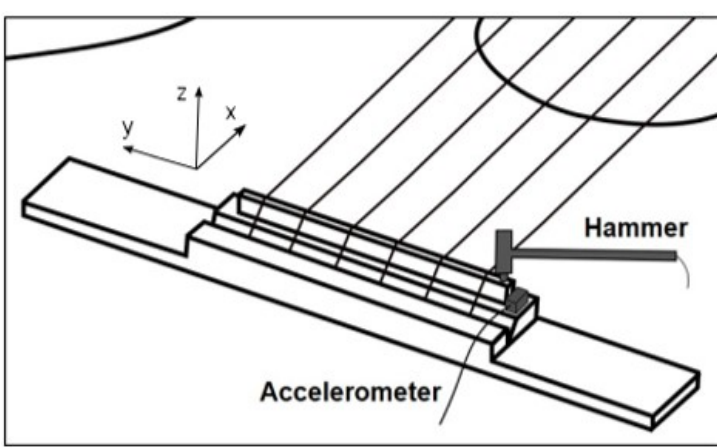

(a)

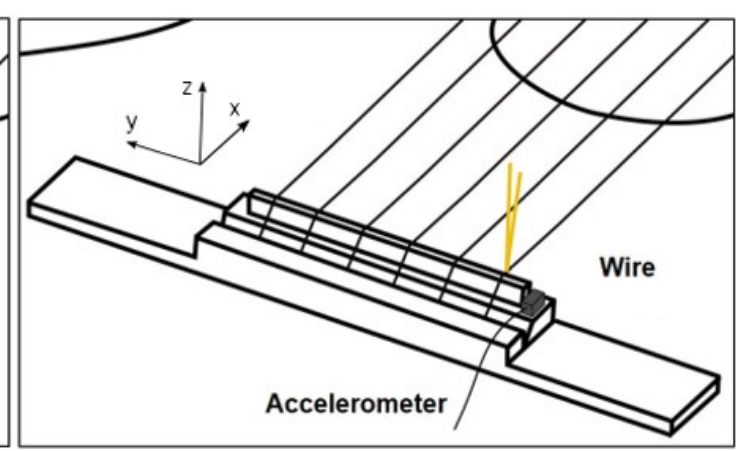

(b)

Figure 1.19: Setups used for mobility measurements at the bridge of string instruments using (a) the hammer technique and (b) the wire technique.

Mobility matrices measured at the string/bridge contact points of string instruments are usually used to feed models for sound synthesis [?, ?, ?]. The classical method used for measuring these transfer functions is based on the so-called hammer method: an impulse force is imparted at the point in the direction of interest by means of a miniature impact hammer and the resulting acceleration is measured by a laser vibrometer or a lightweight accelerometer mounted on the bridge. Figure ?? depicts a typical experimental setup used for measuring mobilities at the bridge of classical guitars using hammer and accelerometer.

\subsection{The wire-breaking method}

Although the wire technique can be used for different types of excitation (see Subsection ??), this paper focuses on the use of this technique for mobility measurement at the bridge of string instruments. In the rest of this paper, therefore, wire-breaking method refers to the method that uses a thin copper wire to excite the points where the strings make contact with the bridge. The wire is placed around the string in a position as close as possible to the saddle and then is pulled aside in the direction of interest until it breaks abruptly imparting a step function force to the excitation point. The measurement of the bridge response without the effect of string motion is feasible when the strings are completely damped. Under those conditions, the acceleration response to the wire excitation measured with a miniature sensor 
mounted on the bridge provides a good estimation of bridge mobilities without using any force sensor.

\subsubsection{Equivalence between $v$-impulse response and $a$-step response}

Let us consider a system described by $\mathrm{N}$ degrees of freedom, a mass matrix $\mathbf{M}$, a damping matrix $\mathbf{C}$, a stiffness matrix $\mathbf{K}$, a displacement $N \times 1$ vector $\mathbf{x}(\mathrm{t})$, excited by a force $N \times 1$ vector $\mathbf{f}(\mathrm{t})$. The Laplace transform of the motion equation

$$
\mathbf{M} \ddot{\mathbf{x}}(t)+\mathbf{C} \dot{\mathbf{x}}(t)+\mathbf{K x}(t)=\mathbf{f}(t)
$$

leads to

$$
\left.X(s)=\left[s^{2} \mathbf{M} \ddot{\mathbf{x}}+s \mathbf{C}+\mathbf{K}\right]^{-1} \mathbf{f}(s)+\left[s^{2} \mathbf{M}+s \mathbf{C}+\mathbf{K}\right]^{-1}((s \mathbf{M}+\mathbf{C})) \mathbf{x}(0)+\mathbf{M} \dot{\mathbf{x}}(0)\right) .
$$

The Laplace transform of the velocity vector resulting from a Dirac excitation applied at one single degree of freedom $\mathbf{f}_{\delta}(t)=\left[\begin{array}{llll}0 & \cdots 0 & \delta(t) & 0 \cdots 0\end{array}\right]^{\mathrm{T}}$ is given by

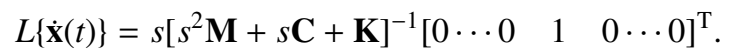

The excitation force resulting from the wire break at one degree of freedom can be represented as a step

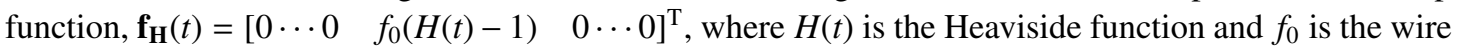
force amplitude. The Laplace transform of the acceleration resulting from $\mathbf{f}_{\mathbf{H}}(t)$ is written as

$$
L\{\ddot{\mathbf{x}}(t)\}=s\left[s^{2} \mathbf{M}+s \mathbf{C}+\mathbf{K}\right]^{-1}\left[\begin{array}{llll}
0 & f_{0} & 0 \cdots 0
\end{array}\right]^{\mathrm{T}} .
$$

The right-hand sides of Equations ?? and ?? are equal if the wire force $f_{0}$ is unitary. As a consequence, the velocity resulting from a Dirac excitation is equivalent to the acceleration response resulting from a unitary step excitation. Thus, the mobility of the system can be obtained from the Fourier transform of the acceleration response resulting from a step force excitation, divided by the wire force as follows:

$$
Y(\omega)=\frac{A(\omega)}{f_{0}} .
$$

\subsubsection{Experimental setup}

All the measurements reported in this paper were performed in the same laboratory environment. The results presented in Section ?? regard to measurements performed on a classical guitar. The instruments were placed in fixed positions using a guitar stand. The instruments were hung by the head and fixed on two of the stand feet using modelling clay so that the contact between the stand and the body only occurred at two points. Before any measurement, the strings were tuned to their usual static tensions. All the measurements were carried out with damped strings.

For measurements using the hammer method, the force signal was provided by a miniature impact hammer PCB Piezotronics 086E80 whose head was mounted on a flexible beam clamped at its extremity. Such setup is a convenient way to control precisely the impact location and to avoid multiple hits. The impact was exerted on the saddle, as close as possible to the point where the E-string makes contact, as shown in Figure ??a.

For measurements using the wire-breaking method, the step force excitation was provided by a thin copper wire with a diameter of $0.1 \mathrm{~mm}$ placed around the E-string in a position very close to the saddle (see Figure ??b). For both hammer and wire-breaking methods, acceleration signals were collected by 
a lightweight accelerometer PCB Piezotronics 352C23 (mass $0.2 \mathrm{~g}$ ) mounted on the bridge, close to the excitation point.

\subsection{Results and discussion}

\subsubsection{Mobilities obtained with the hammer and wire-breaking methods}

Typical mobility measurements at the bridge of banjos, Brazilian guitars, classical guitars and violins obtained by the hammer method are compared in Figure ??, which highlights the difference of profiles of those four instruments. All the mobilities are characterized by numerous resonances, which induce variations around the averaged value over the useful frequency range. The mean mobility and the modal density are important features of the instrument soundbox [?]. Since the soundboard of the banjo is a membrane, its mobility is the highest up to $1500 \mathrm{~Hz}$. On the other hand, the violin mobility is amplified in the vicinity of $2500 \mathrm{~Hz}$, presenting the highest values: this feature is often referred to as the bridge hill $[?, ?, ?]$. The guitar soundboards (classical and Brazilian) have been shown to behave as plate-like systems: their mean mobilities and the modal densities are nearly independent on the frequency. This property is the one of a plate, whose equivalent parameters can be computed ( $c f .[?])$.

Figure ?? shows the comparison between calibrated and uncalibrated mobilities, from 0 to 2000 $\mathrm{Hz}$, obtained with the hammer and wire-breaking methods, respectively. Figure ?? shows the same measurements in a frequency range from $2000 \mathrm{~Hz}$ to $7000 \mathrm{~Hz}$. In general, both curves present similar patterns except for the difference in level, which is about $10 \mathrm{~dB}$ in the overall frequency range. These discrepancies are expected since the measurements obtained by the wire method are not calibrated, i.e. the factor $f_{0}$ is not taken into account. It is also observed that, at frequencies higher than $4000 \mathrm{~Hz}$, the hammer method leads to noisier results, revealing another advantage of using the wire-breaking method in such frequency range. The results presented in Figures ?? and ?? show that the determination of the factor $f_{0}$ is crucial to validate mobility measurements obtained with the wire-breaking method.

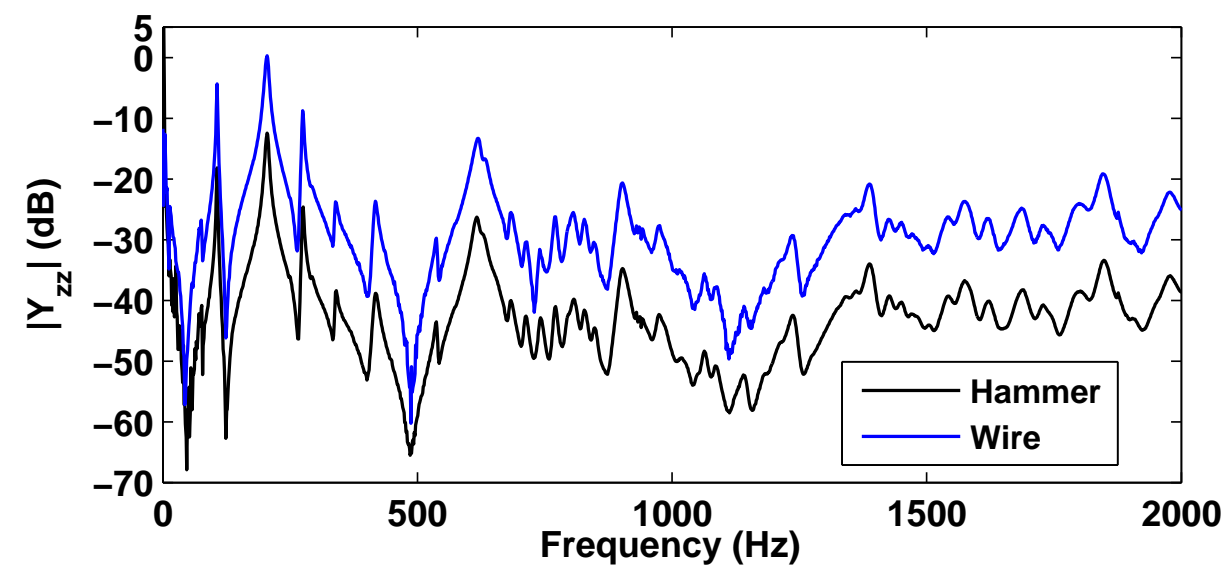

Figure 1.21: Mobility curves, from 0 to $2000 \mathrm{~Hz}$, measured using the hammer (black line) and wire-breaking (blue line) methods. The $\mathrm{dB}$ scale reference is $1 \mathrm{~m} \cdot \mathrm{s}^{-1} \cdot \mathrm{N}^{-1}$ (For interpretation of the references to color in this figure legend, the reader is referred to the web version of this article.) 


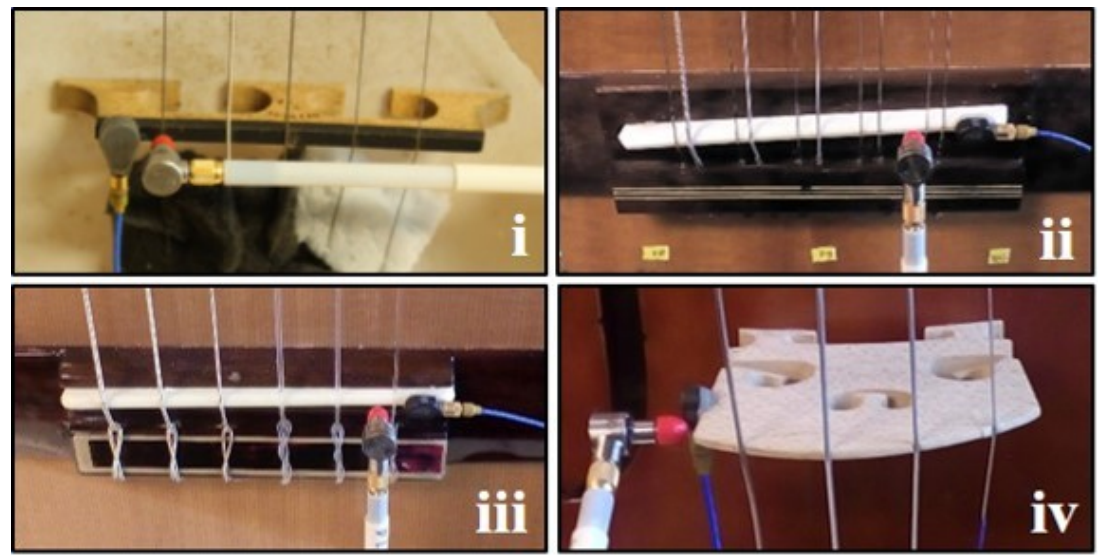

(a)

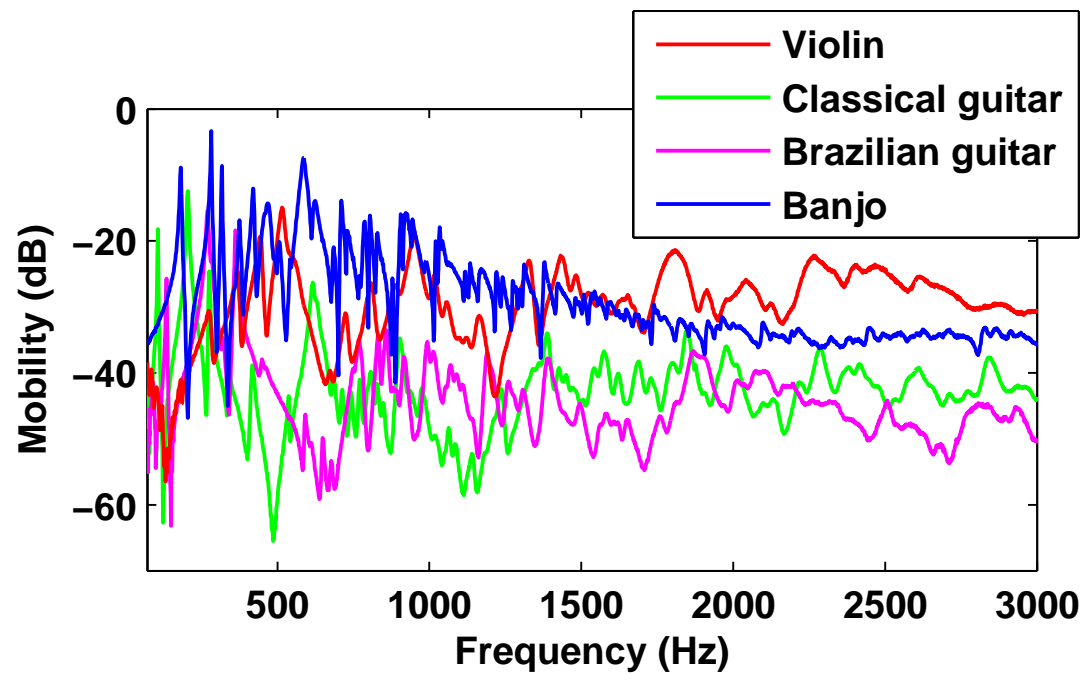

(b)

Figure 1.20: (a) Experimental setups and (b) respective mobility curves measured using the hammer method at the bridge of different instruments: i) banjo (blue line), ii) viola caipira (magenta line), iii) classical guitar (green line), viola caipira (magenta line), iv) violin (red line). The $\mathrm{dB}$ scale reference is $1 \mathrm{~m} \cdot \mathrm{s}^{-1} \cdot \mathrm{N}^{-1}$. (For interpretation of the references to color in this figure legend, the reader is referred to the web version of this article.) 


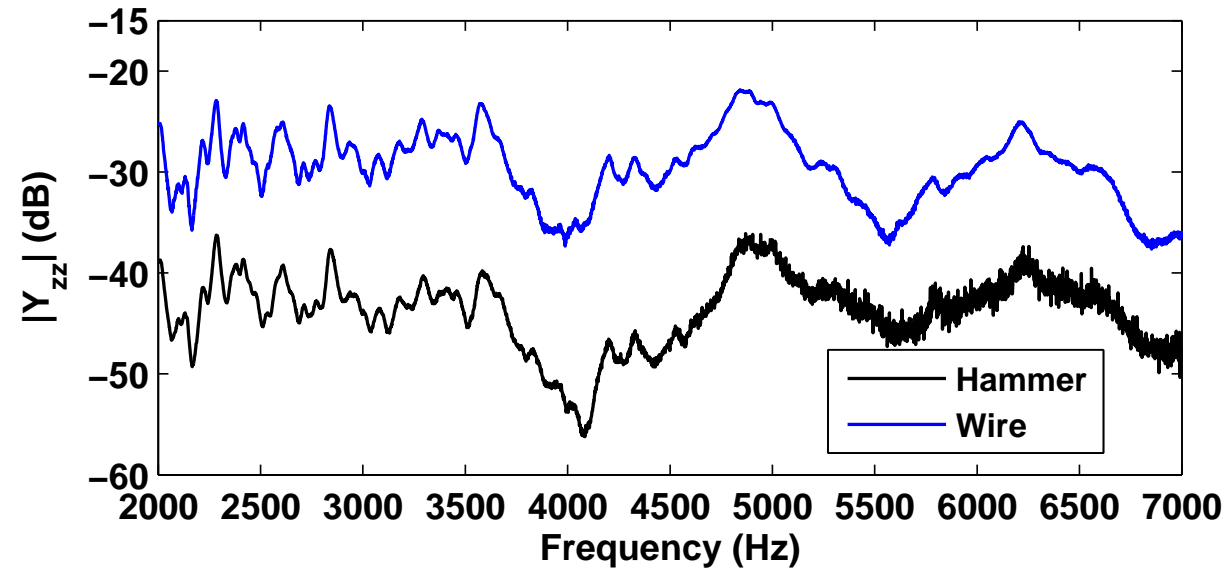

Figure 1.22: Mobility curves, from $2000 \mathrm{~Hz}$ to $7000 \mathrm{~Hz}$, measured using the hammer (black line) and wirebreaking (blue line) methods. The $\mathrm{dB}$ scale reference is $1 \mathrm{~m} \cdot \mathrm{s}^{-1} \cdot \mathrm{N}^{-1}$. (For interpretation of the references to color in this figure legend, the reader is referred to the web version of this article.)

\subsubsection{Repeatability of the wire-breaking method}

In order to assess the repeatability of the wire-breaking method, five mobility curves were measured under the same measurement conditions and compared in Figure ??. It can be observed that all the curves have substantially the same profile, which confirms the satisfactory repeatability of the method. Since the breaking force $f_{0}$ is expected to be invariable for samples from the same reel and the choice of the excitation angles are controllable, the method allows reproducible measurements in different environments, by manipulation of different operators.

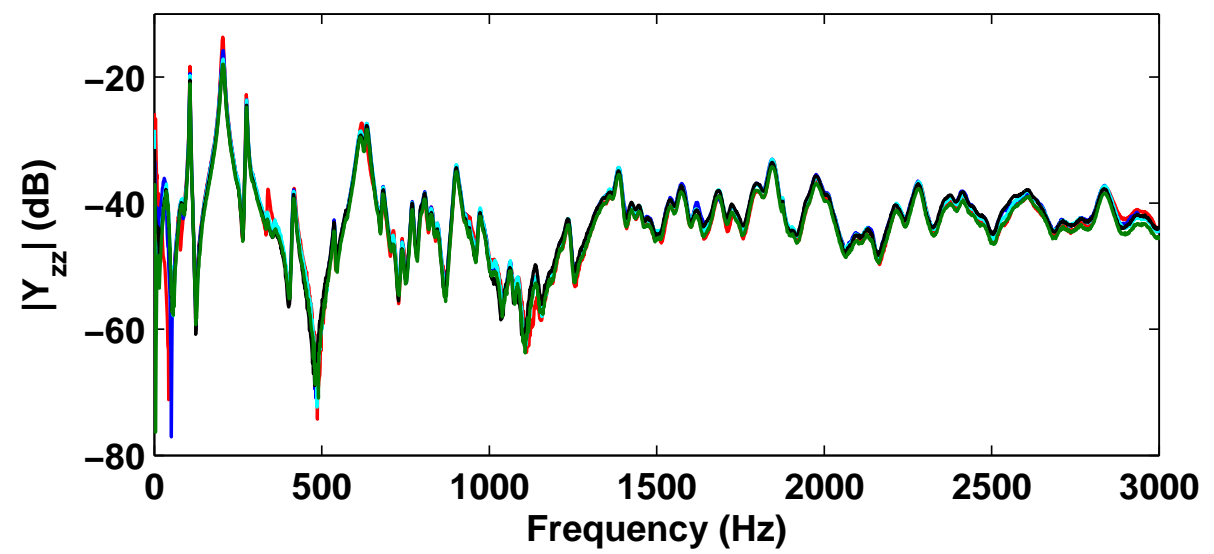

Figure 1.23: Five mobility curves measured under the same conditions using the wire-breaking method. The $\mathrm{dB}$ scale reference is $1 \mathrm{~m} \cdot \mathrm{s}^{-1} \cdot \mathrm{N}^{-1}$. (For interpretation of the references to color in this figure legend, the reader is referred to the web version of this article.)

\subsubsection{Influence of the wire diameter}

The wire thickness is directly related to the magnitude of the wire breaking force $f_{0}$ so that the choice of the wire diameter is determinant in the reliability of the measurements. Thicker wires may provide 
an excitation force $f_{0}$ enough to move the instrument body while the wire is pulled aside, which leads to distorted measurements. Conversely, thinner wires may provide a low signal to noise ratio resulting in unreliable measurements. Figure ?? shows a comparison between mobility curves measured using wires of three different diameters. Although measurements using the $150 \mu \mathrm{m}$ wire have shown to be the most satisfactory in terms of signal-to-noise ratio, the provided excitation force was too high so that the guitar moved from the support. On the other hand, as can be shown in the same figure, the $56 \mu \mathrm{m}$ wire provided the noisier results. The $100 \mu \mathrm{m}$ wire is, therefore, a good choice since it provides a good signal-to-noise ratio and a suitable force for the measured instrument.

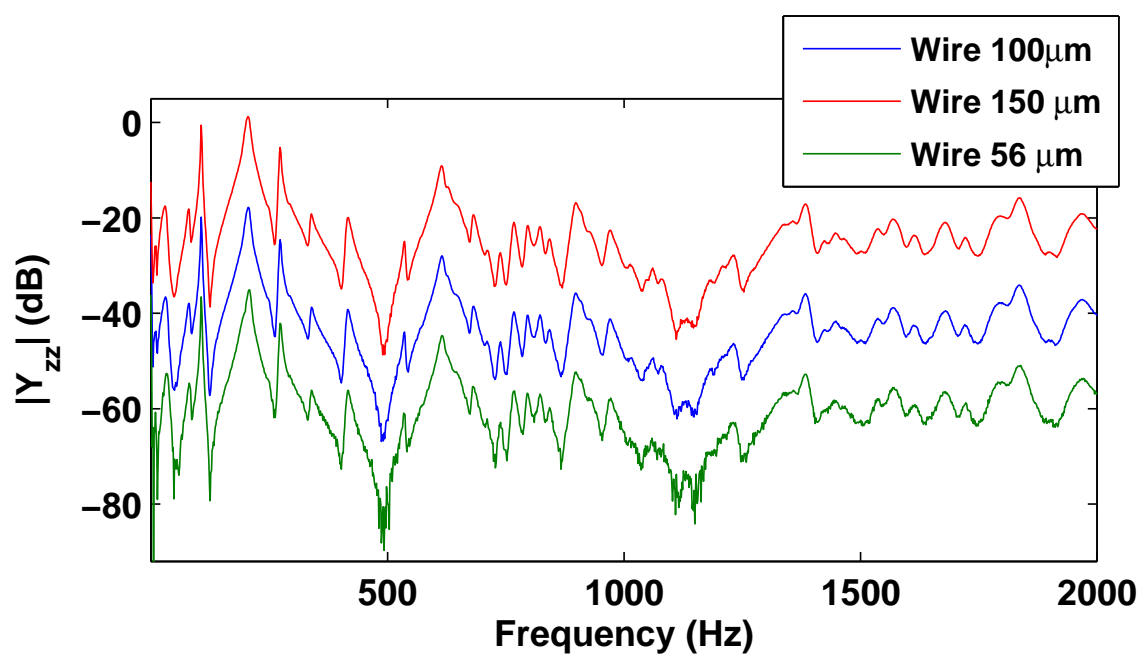

Figure 1.24: Mobility curves measured at the bridge of a classical guitar using wires of 3 different diameters: $56 \mu \mathrm{m}$ (green line), $100 \mu \mathrm{m}$ (blue line) and $150 \mu \mathrm{m}$ (red line). The green and blue curves are offset on the $\mathrm{y}$-axis so that the comparison between the curves is feasible. The $\mathrm{dB}$ scale reference is $1 \mathrm{~m} \cdot \mathrm{s}^{-1} \cdot \mathrm{N}^{-1}$. (For interpretation of the references to color in this figure legend, the reader is referred to the web version of this article.)

\subsection{Calibration of the wire-breaking method}

Wire measurements require only one acceleration sensor for measuring mobilities on the bridge of the instruments. As a consequence, the breaking force $f_{0}$ has to be determined in a preliminary phase in order to compute calibrated mobilities.

\subsubsection{Measuring the wire-breaking force}

Figure ?? shows the experimental setup used for measuring the wire-breaking force. The measurements consist in threading the wire through a rigid holder attached to the head of an impact hammer PCB Piezotronics 086C03, while the opposite hammer end is clamped onto a flat surface. In this way, the magnitude of the force measured by the hammer while the wire is pulled until it breaks is equivalent to the force exerted on the string. The value of $f_{0}$, therefore, is given by the maximum magnitude of the force curve measured in function of time, named the wire-breaking force curve. 


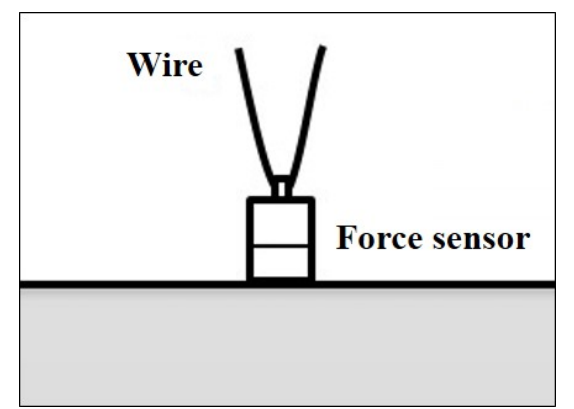

Figure 1.25: Experimental setup used for measuring the wire-breaking force: the wire is attached to a fixed force sensor and pulled until it breaks.

Figure ?? shows a typical wire-breaking force curve. For the sake of better visualization the signal of the measured force was inverted. At first, an upward force region is observed, which corresponds to the time interval that the wire is stretched. Then, the wire breaks and the measured force falls abruptly since no tension is exerted by the wire.

Finally, the measured force features a damped oscillatory behavior that fades out progressively. Figure ?? shows the comparison between 10 measures of the wire-breaking force curves obtained under the same conditions. Although all the curves have similar profiles, small differences can be observed, which can be due to slight variations of the gesture made by the operator while pulling the wire. Since the factor $f_{0}$ is given by the maximum magnitude of the wire-breaking force curves, a value of $f_{0}=(4.32 \pm 0.14) N$ is obtained as indicated in Figure ??. Noting that the wire-breaking setup consists of two strands that equally share the pulling load, the maximum force withstood by the strand that breaks is $f_{0} / 2$, which corresponds to a stress of $275 \mathrm{MPa}$. This value is in line with typical ultimate tensile strength reported for enamelled copper wire [?]. 


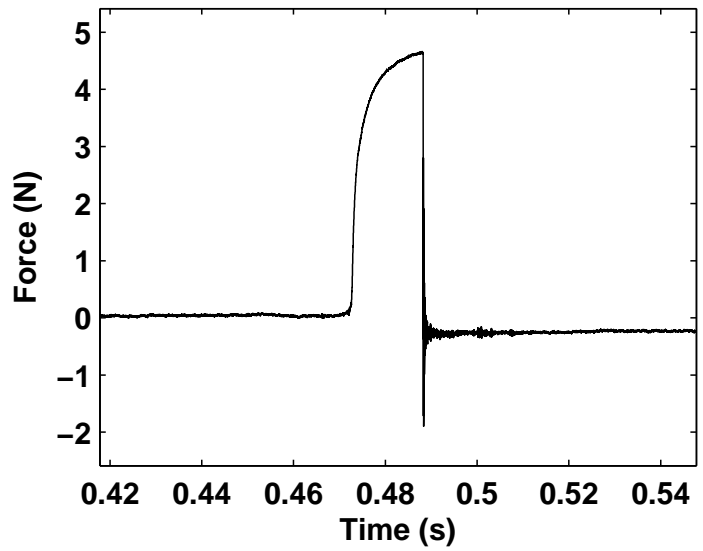

(a)

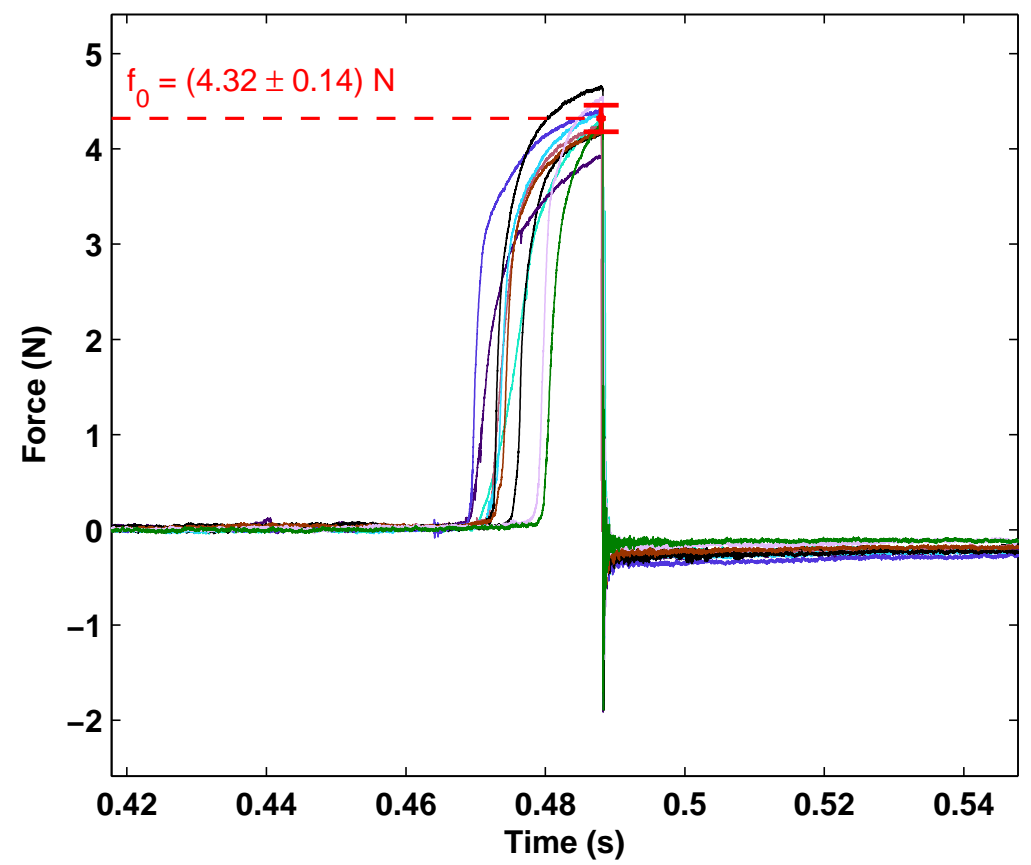

(b)

Figure 1.26: (a) Typical measure (b) and ten measures of the breaking force curve of a $100 \mu \mathrm{m}$ wire. (For interpretation of the references to color in this figure legend, the reader is referred to the web version of this article.)

Figure ?? allows to compare the bridge mobilities measured with the hammer method and the wirebreaking method after calibration via the procedure described above. It can be observed a satisfactory agreement between both curves, which indicates that the experimental procedure used for measuring the wire-breaking force curves provides a suitable calibration for the wire-breaking method. 


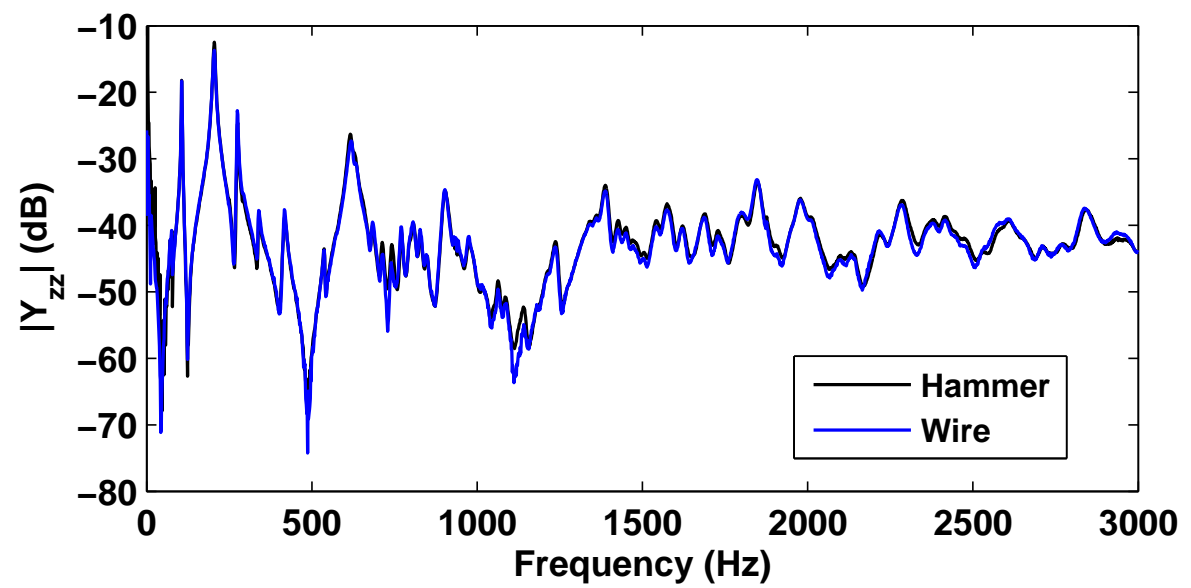

Figure 1.27: Mobility curves measured using the hammer method (black line) and the wire-breaking method (blue line) after calibration. The $\mathrm{dB}$ scale reference is $1 \mathrm{~m} \cdot \mathrm{s}^{-1} \cdot \mathrm{N}^{-1}$. (For interpretation of the references to color in this figure legend, the reader is referred to the web version of this article.)

\subsection{Application: Roving Wire-Breaking Technique}

\subsubsection{High resolution modal analysis}

Since impact hammer measurements can be replaced by breaking wire measurements, can a modal analysis procedure be performed on these measurements with sufficient precision? To address this issue, a "Roving Wire-Breaking Technique" is defined and is carried out on a Brazilian guitar, the viola caipira, which is composed of 5 pairs of strings (see Figure ??). The aim is to determine the body modal parameters, i.e frequencies, damping coefficients and mode shapes components at the 5 coupling points (denoted 1 to 5) in both directions (out-of-plane direction denoted $\mathrm{z}$ and in plane direction $\mathrm{y}$ ).

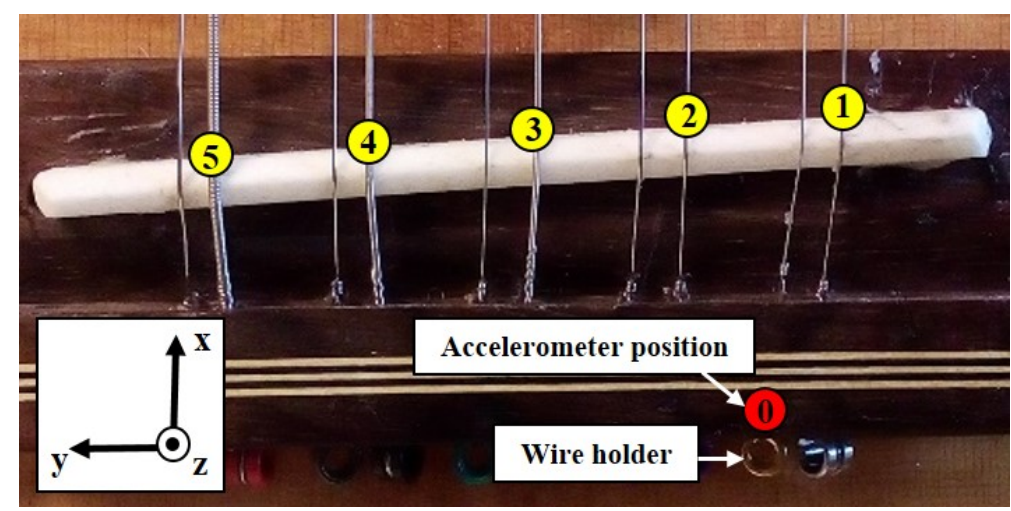

Figure 1.28: Scheme used for estimation of mode shapes at the bridge of the viola caipira using the wirebreaking method. Collocated mobility is measured at point 0 (in red), where the accelerometer is fixed; the wire is . Points 1 to 5 (in yellow) are excited using the wire technique in the $z$ and $y$ directions. (For interpretation of the references to colour in this figure legend, the reader is referred to the web version of this article.)

At the bridge of a string instrument, it is common that the accelerometer cannot be placed exactly on one of the coupling points. To circumvert this difficulty, a reference point denoted 0 is selected in such a 
way that a collocated measurement at this point is possible. Responses resulting from the wire-breaking excitation are measured by a miniature accelerometer located at point 0 . By roving the wire between the 6 measurement locations ( 5 coupling points +1 reference point) and by orienting the wire in both directions $z$ and $y$, a set of 12 responses can be measured. Dividing by the calibration factor $f_{0}, 12$ impulse responses $y_{i j}(t)$ can be obtained, where subscript $i$ denotes the response degree of freedom (chosen here as $i=0 z$ ) and subscript $j$ denotes the excitation degree of freedom. For example $y_{0 z, 1 y}$ is the impulse response measured at point 0 in the direction $z$ resulting from a wire break at point 1 and in the direction $y$. The modal analysis is performed in two steps.

- The first step concerns the identification of modal frequencies and damping coefficients using the high resolution technique ESPRIT described in [?, ?]. This technique is based on the time-domain representation of the signal $s(t)$ as a sum of complex exponentials, whose discrete representation $s[n]$ is written as:

$$
s[n]=\sum_{k=1}^{2 K} a_{k} e^{-\alpha_{k}^{\prime} n} e^{j\left(2 \pi f_{k}^{\prime} n+\varphi_{k}\right)}=\sum_{k=1}^{2 K} b_{k}\left(z_{k}\right)^{n},
$$

where the $K$ modes are characterized by their dimensionless modal frequencies $f_{k}^{\prime}$, and their damping factors $\alpha^{\prime}$, and are associated to amplitudes $a_{k}$ and phases $\varphi_{k}$. The identification procedure consists in estimating the poles $z_{k}=e^{-\alpha_{k}^{\prime}+j 2 \pi f_{k}^{\prime}}$ from which a collection of modal frequencies and modal damping coefficients can be obtained from

$$
f_{k}=\frac{\arg \left(z_{k}\right)}{2 \pi} F_{s} \quad \text { and } \quad \xi_{k}=\frac{-F_{s} \ln \left|z_{k}\right|}{2 \pi f_{k}}
$$

where $F_{s}$ is the sampling frequency. The pole identification is performed using the ESPRIT algorithm, which is based on the decomposition of the input vector space onto two orthogonal subspaces, namely the signal and noise subspaces [?].

- The second step consists in estimating the modeling order and the mode shape components based on a fit in the frequency domain. For this purpose, the mobility $Y_{i j}(\omega)$ is computed as the Fourier Transform of the response $y_{i j}(t)$. The modal model of $Y_{i j}(\omega)$ is written as:

$$
Y_{i j}(\omega)=\sum_{k=1}^{K} A_{k} H_{k}(\omega)
$$

with

$$
H_{k}(\omega)=\frac{j \omega}{\omega_{k}^{2}-\omega^{2}+j 2 \xi_{k} \omega_{k} \omega} \quad \text { and } \quad A_{k}=\Phi_{i k} \Phi_{j k},
$$

where $\Phi_{j k}$ and $\Phi_{j k}$ are the $i$ th and $j$ th components of the $k$ th mode shape (mass normalized). The amplitudes $A_{k}$ such that the modal sum of Eq. (??) best fits the measured mobility can be found by solving a least squares problem. Two aspects must be considered to properly perform this least squares estimation. Firstly, it should be noted that the amplitudes $A_{k}$ are real, whereas $H_{k}(\omega)$ is complex-valued. Secondly, in the particular case of the collocated mobility $Y_{0 z, 0 z}(\omega)$, the amplitudes $A_{k}=\phi_{0 z, k} \phi_{0 z, k}$ are positive. To satisfy these two constraints, the estimation of amplitudes is first performed on the collocated mobility using a non-negative least squares (NNLS) procedure. The problem is expressed as

$$
\min _{\mathbf{x}}\|\mathbf{C x}-\mathbf{d}\|_{2}^{2} \quad \text { with the constraint } \quad x_{k} \geq 0 \quad \forall k,
$$


where $\mathbf{x}=\left[\begin{array}{lllll}A_{1} & \ldots & A_{k} & \ldots & A_{K}\end{array}\right]^{\mathrm{T}}$ is the vector of unknown modal amplitudes,

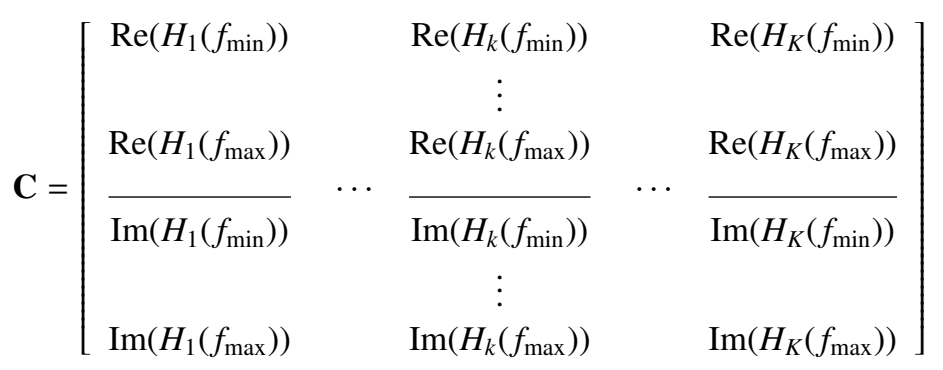

is a $2 N_{\text {freq }} \times K$ matrix whose columns form a basis of unitary modal responses and

$$
\mathbf{d}=\left[\begin{array}{c}
\operatorname{Re}\left(H_{0 z, 0 z}\left(f_{\min }\right)\right) \\
\vdots \\
\operatorname{Re}\left(H_{0 z, 0 z}\left(f_{\max }\right)\right) \\
\operatorname{Im}\left(H_{0 z, 0 z}\left(f_{\min }\right)\right) \\
\vdots \\
\left.\operatorname{Im}\left(H_{0 z, 0 z} f_{\max }\right)\right)
\end{array}\right]
$$

is a $2 N_{\text {freq }} \times 1$ vector containing the measured mobility. The splitting of the unitary modal responses $H_{k}(\omega)$ and the measured mobility $H_{0 z, 0 z}(\omega)$ into their real and imaginary parts is necessary to enforce that the estimated amplitude $A_{k}$ are real. A characteristic of the NNLS procedure is that the solution $\mathbf{x}$ consists of two subsets, one containing only strictly positive values and the other containing only zeros. Consequently, the procedure intrinsically provides a model order selection, since it does not use all poles identified by ESPRIT to fit the measured response. In the present application of the method, 47 modes were retained out of 165 candidate modes. Once the modal amplitudes of the collocated mobility have been estimated, those of the cross mobilities can be obtained by solving a standard least squares problem, which has the same form as Eq. (??) without the non-negative constraint. The identification of modal amplitudes for each cross-mobility measurement allows to determine the $z$ - and $y$-components of mode shapes at the different coupling points $\Phi_{j, k}=A_{j, k} / \Phi_{0 z, k}(j=1 z, 1 y, 2 z, 2 y \ldots)$.

Figure ?? compares the collocated mobility $H_{0 z 0 z}$ reconstructed from the NNLS solution to the measured mobility. A good fit is obtained in the frequency band of interest. The cross mobility $H_{0 z, 0 y}$ reconstructed from the standard least squares solution using the same modes is compared to the measured mobility in Figure ??. The signal-to-noise ratio of this measurement is lower due to the smaller amplitude of the soundboard vibrations in the $y$-direction. However, the reconstructed mobility overall well follows the measured mobility.

\subsubsection{Synthesis of the mobility matrix}

An example of reconstruction of the full mobility matrix at one coupling point is shown in Figure ??. Note that a matrix concerns degrees of freedom whose physical access is difficult or impossible. Since the mobility matrix provide a full characterization of the instrument body at the coupling point, such a 


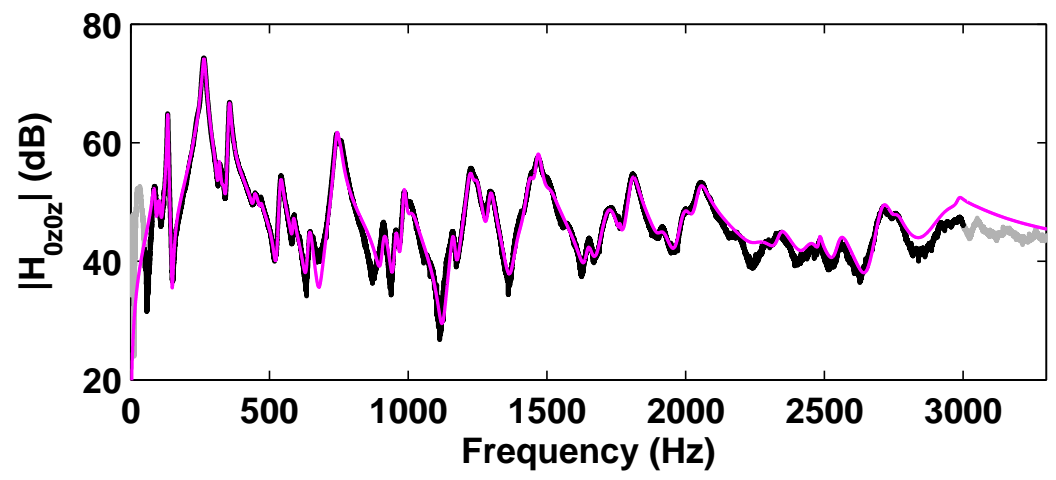

(a)

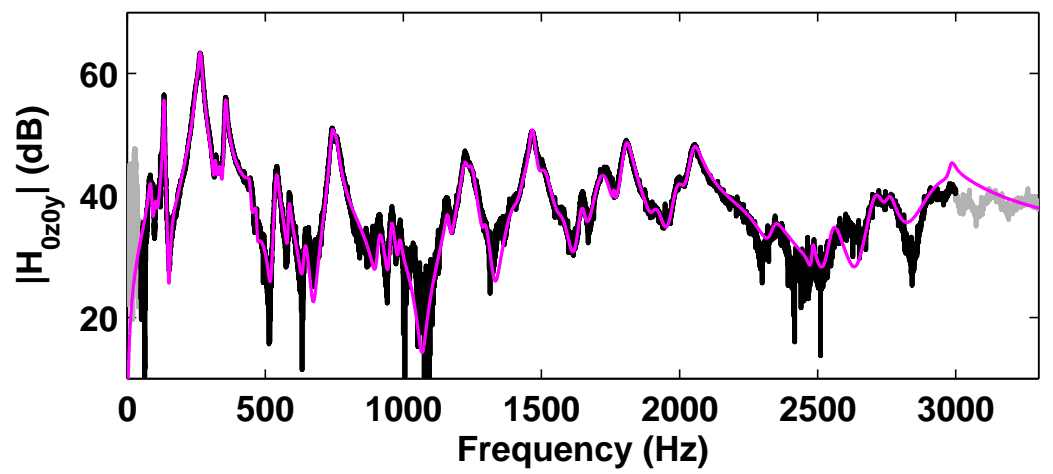

(b)

Figure 1.29: Modulus of (a) the direct mobility $H_{0 z 0 z}$ and (b) the cross mobility $H_{0 z 0 y}$ at the reference point 0 . Black curve: measurement using the wire-breaking method. This curve is shaded below $f_{\min }=50 \mathrm{~Hz}$ and above $f_{\max }=3000 \mathrm{~Hz}$ to highlight the frequency range considered for modal amplitudes estimation. Magenta curve: reconstruction using 47 real modes obtained by the ESPRIT/NNLS procedure.(For interpretation of the references to color in this figure legend, the reader is referred to the web version of this article.)

data is a useful input data for sound synthesis tools based on hybrid techniques.

\subsubsection{Identification of mode shapes}

Figure ?? shows a selection of identified mode shapes at the bridge in the out-of-plane direction. Operating deflection shapes (ODS) of the instrument body at peak frequencies close to these modes are shown in Figure ?? for comparison purpose. These ODS were obtained by exciting the bridge with an automatic impact hammer (Maul-Theet vImpact-60) and measuring the resulting velocity at numerous locations using a scanning laser vibrometer (Polytech PSV-500). The first mode shape identified by the procedure corresponds to a rigid-body mode of the instrument (see ODS a). Modes shape 3 and 7 are those of the $\mathrm{A} 0$ and $\mathrm{T} 1$ modes, which are the lowest modes significantly contributing to sound radiation of a guitar. They correspond to coupled motion of the top plate (see ODS b and c) and air piston in the soundhole through the stiffness of the air cavity. The resulting motion at the bridge involves inphase, piston-like motion of the 10 coupling points. Mode 10 corresponds to the T2 mode, which is related to a top plate mode shape with a single longitudinal nodal line crossing bridge (see ODS d). As 


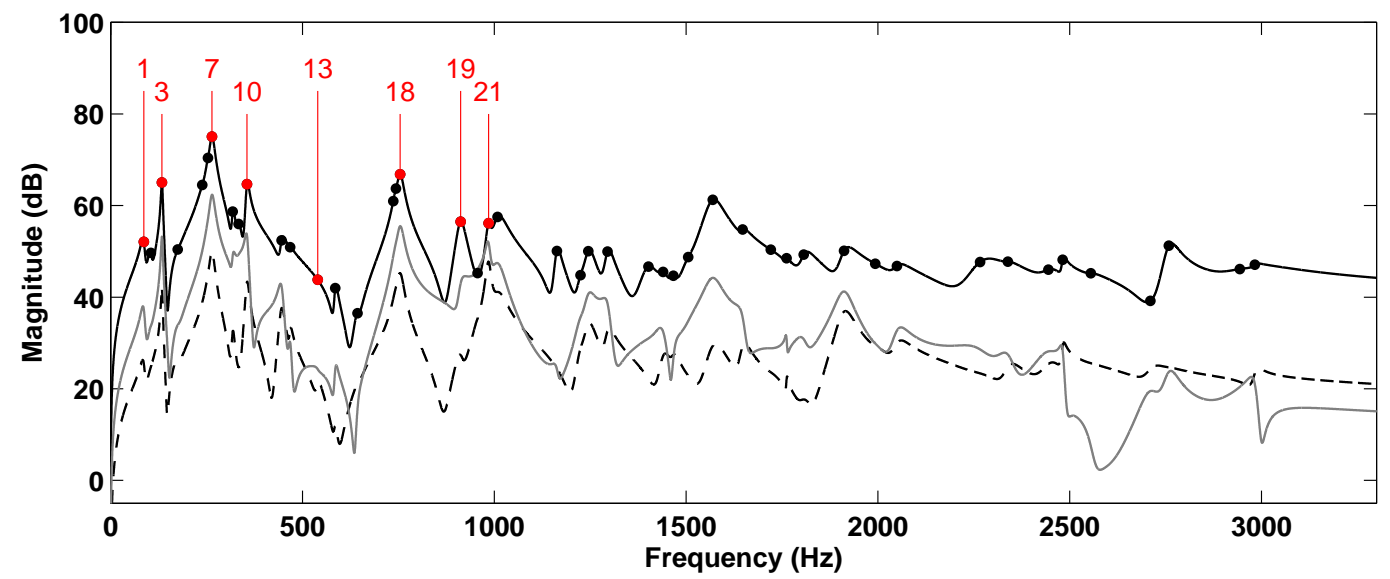

Figure 1.30: Modulus the mobility matrix terms at coupling point 1 reconstructed using the modal parameters identified by the method. Black continuous line: direct out-of-plane mobility $H_{1 z 1 z}$, black dashed line: direct in-plane mobility $H_{1 y 1 y}$, gray continuous line: cross mobility $H_{1 z 1 y}$. The dots on the out-of-plane mobility indicates the modes identified by the method and used for the mobility synthesis. The mode shapes of modes pointed out by their number are shown in Figure ??.

a result, it is the first mode where out-of-phase motion between coupling points occurs, namely between the treble and bass sides of the bridge. Although this may come as a surprise, piston-like motion of the bridge again occurs at some higher modal frequencies (e.g. modes 18 and 19). This can be understood when considering the related ODS (see ODS $\mathrm{f}$ and $\mathrm{g}$ ), where the top plate exhibits more complex modal patterns but the bridge is not crossed by any nodal line. In contrast, mode 21 involves more rapid spatial variations of amplitude along the bridge, which is constrained to follow a motion of top plate involving more closely spaced longitudinal nodal lines (see ODS h). The knowledge of these relative amplitudes and phase relationships for the different modes can be useful in a model to account more accurately for sympathetic vibration between all strings.

\subsection{Conclusion}

This paper has investigated the capability of the wire-breaking method to accurately obtain the mobilities transfer functions at the bridge of a string instruments. Since no force sensor is required, this methodology is a low cost and well-adapted procedure for measurements in the environment of instrument maker workshop. The method was shown to be repeatable and provided results in reasonable agreement with the classical hammer method. A calibration method for mobilities obtained from wirebreaking measurement was proposed and validated. Finally, a modal analysis of the mobility curves measured at the bridge using the "Roving Wire-Breaking Technique" allowed the estimation of natural frequencies, damping factors and mode shapes at the string/bridge coupling points using a high resolution modal analysis. Such results can be used to feed sound synthesis models. 


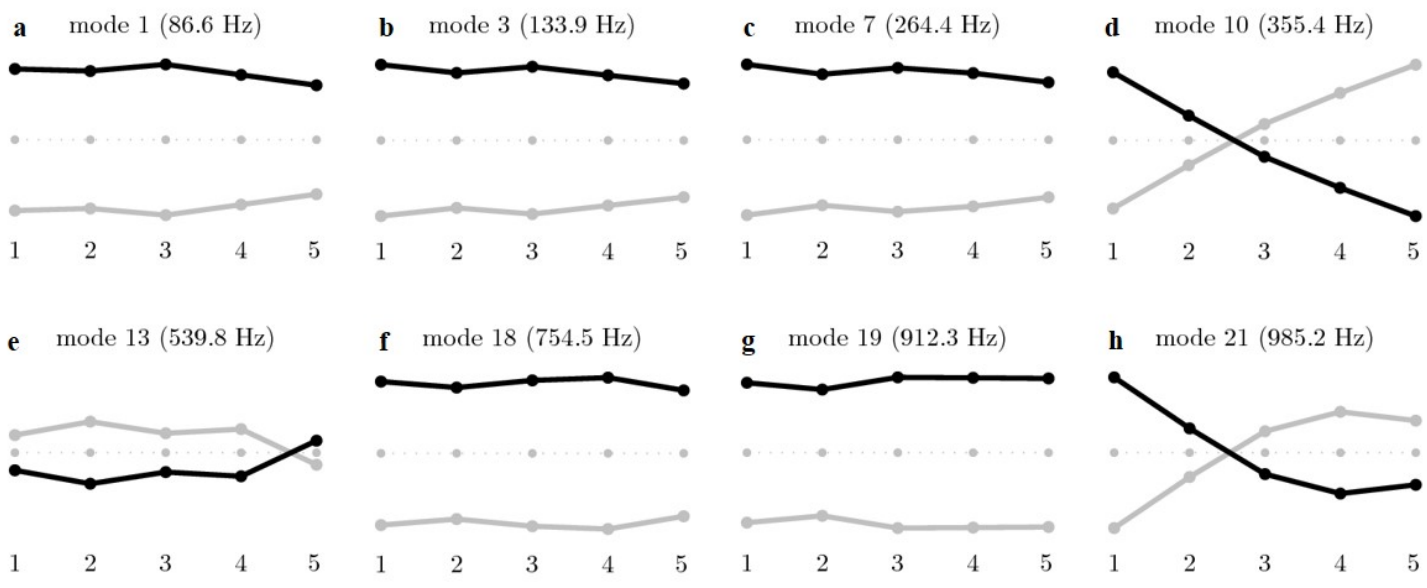

(a) Mode shapes at the bridge saddle.

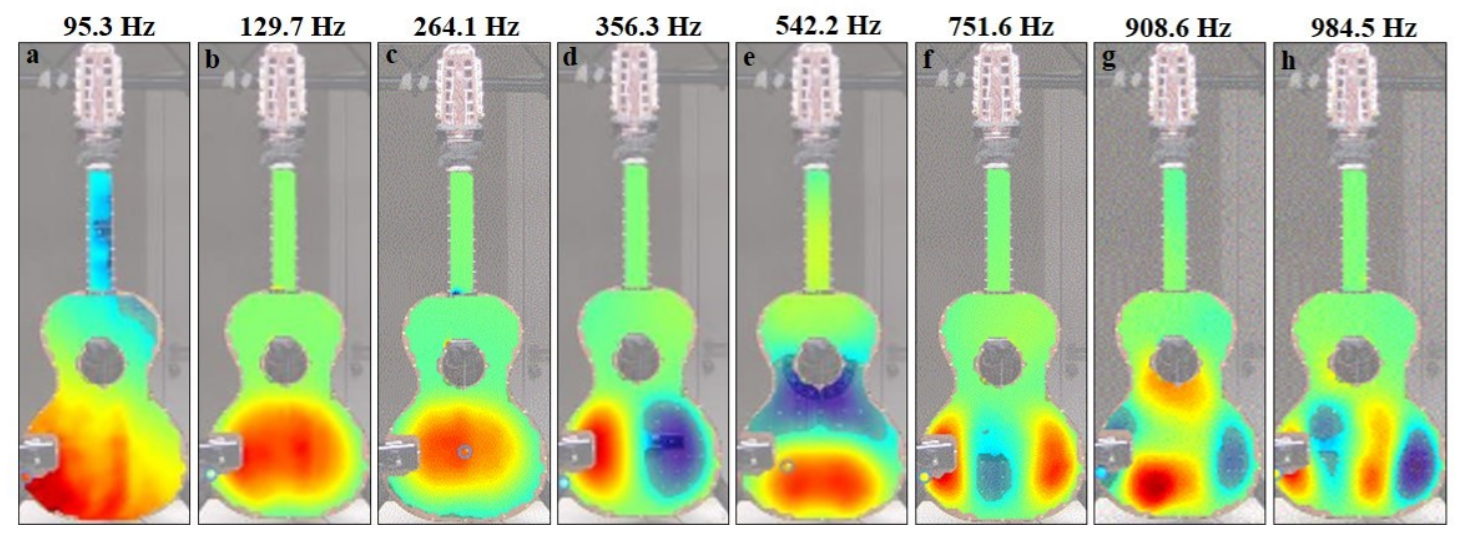

(b) Global operating deflection shapes.

Figure 1.31: (a) Mobility curve synthesized using estimated modal parameters; (b) examples of mode shapes at the bridge saddle and (c) corresponding operating deflection shapes of the viola caipira body. 


\subsection{Summary}

An experimental study of the viola caipira has been presented in this chapter where different aspects were approached:

- The high speed camera analysis also revealed existence of shocks between strings located in the same pair;

- The analysis of the sound resulting from the viola caipira pluck when all the strings are free to vibrate revealed the existence of string sympathetic resonances, which are perceived as a sound halo, constituting an important sound feature of the instrument;

- The modal analysis of the viola caipira soundboard carried out by the classical hammer method revealed some differences and similarities with the classical guitar: the first four modes of the viola caipira soundboard resemble those of the classical guitar while frequencies are moderately higher since the resonance box of the instrument is relatively smaller. In addition, the viola caipira soundboard, like classical guitar soundboards have been shown to plate-like systems since their mean mobilities are nearly independent on the frequency;

- Measurements using a scanning laser vibrometer and an automatic impact hammer have been performed to obtain the ODSs of the front of the viola caipira. At a set of resonance peaks the obtained ODSs gives results close to those obtained with the classical modal analysis using impact hammer and accelerometer;

- Bridges mobilities have been measured using the wire-breaking method, which is simple to use and inexpensive since it does not require the use of a force sensor. Combined with a high-resolution modal analysis (ESPRIT method), these measurements enabled to determine the modal shapes at the string/body coupling points and thus to characterize the instrument. 



\section{Chapter 4}

\section{PhysicAl MODELLING AND SOUND SYNTHESIS OF THE viola caipira.}

The experimental study presented in Chapter ?? revealed that the interaction between the viola caipira strings may occur in two different ways: through the motion of the bridge, whereby all the strings are coupled, and through successive collisions of strings located in the same pair. This chapter aims at presenting a physical modelling for sound synthesis of the viola caipira able to reproduce both phenomena, which, undoubtedly, contribute to the sound particularity of the instrument.

The text below is structured around the article entitled "Collisions in double string plucked instruments: physical modelling and sound synthesis of the viola caipira", submitted to the Journal of Sound and Vibration in November 2017. In that article is presented a modal-based model comprising ten strings with non-planar motions coupled with the body. The model includes string/string collisions and combines an analytical approach to describe the vibrations of strings and experimental data describing the body. Simulations in the time domain reveal the main sound characteristics of the viola caipira.

In order to further explore the sound synthesis model developed in the article, complementary simulations are presented in Complement I. 


\title{
Collisions in double string plucked instruments: physical modelling and sound synthesis of the viola caipira
}

\author{
${ }^{1,2}$ G. O. Paiva, ${ }^{1}$ F. Ablitzer, ${ }^{1}$ F. Gautier and ${ }^{2}$ J. M. C. dos Santos \\ ${ }^{1}$ Université du Maine, LAUM, Avenue Olivier Messiaen, 72085 Le Mans cedex 9, France \\ ${ }^{2}$ University of Campinas, UNICAMP-FEM-DMC, Rua Mendeleyev, 200, CEP 13083-970 Campinas, SP, Brazil
}

\begin{abstract}
The viola caipira is a folk guitar widely used in traditional and modern Brazilian music. It consists, in general, of 10 metallic strings arranged in five pairs, tuned in unison or octave, with the thinnest string located in the middle. An experimental study of the viola caipira pluck by means of a high speed camera reveals some specificities of the instrument. It is found that the instrument is characterized by a double pluck excitation since the two strings of a given pair are plucked successively and rapidly. Collisions between strings arranged in the same pair are identified. A hybrid model, based on a modal approach, is carried out for sound synthesis purposes. It includes 10 strings with non-planar motions coupled with the body and collisions between strings. A finite difference scheme is used to compute the coupling forces at each time-step, which permits a set of sound simulations. The effects of string/string collisions on the viola caipira sounds are identified and discussed. It is found that the model reproduces the main vibroacoustic features of the viola caipira, among which the sympathetic string resonances and the string/string collisions observed in the video analysis.
\end{abstract}




\subsection{Introduction}

Collisions are strongly non-linear phenomena present in various mechanisms of musical instruments. Such phenomena are closely related to the timbre characteristics of many instruments and can occur in two general ways: at a well-defined instant and specific location, as in the hammer/string interaction in pianos, or continuously in time and spatially distributed, as in the bridge/string interaction in typical Indian instruments like the tampura and sitar.

Numerous authors have addressed different types of collisions in string instruments modelling. Such works may be gathered in different groups according to the objects involved: fret/string [?, ?, ?, ?, ?], bridge/string [?, ?, ?, ?], hammer/string [?, ?, ?, ?, ?]. Many works adopt physical models and employ numerical schemes to discretize and solve partial differential equations describing the string motion. Some models rely on finite differences schemes in space and time [?, ?, ?, ?, ?], while others employ modal representations of strings [?, ?, ?, ?, ?]. There are also works that combine modal and non-modal representations using different methodologies [?, ?, ?, ?]. Standard time stepping methods of solution consist in decreasing the step until the simulation achieves convergence with the desired precision. In this case, the choice of a very small time step to calculate accurately non-linear collision forces may lead to high computation times. Alternatively, several authors $[?, ?, ?, ?, ?, ?]$ have used energy-based methods, which enables by means of energy conservation frameworks suitable stability conditions to numerical schemes employed. In addition, digital waveguide methods have been also applied to simulate vibrating strings interacting with obstacles [?,?].

Although numerous types of collision have been investigated in previous works, string/string collisions, to our knowledge, are not reported yet in the literature. Apparently, this is an unexplored subject that can be of considerable interest for the sound synthesis field. String/string collisions are evidently dependent on the spacing between strings and therefore are expected to occur specially in plucked instruments with double strings like lutes, mandolins, Portuguese guitars, viola caipira, etc. On the other hand, it is reasonable to expect that collisions between strings might not occur, or occur less frequently, in instruments where the string spacings are relatively large, like classical guitars.

This paper aims at evidencing experimentally and modelling collision phenomena in double strings of a typical Brazilian guitar known as viola caipira (see Figure ??). A physical model for sound synthesis able to reproduce such collision phenomena is presented and a set of time domain simulations is obtained. Such model uses a modal-based approach and includes 10 strings with non-planar motions coupled with the body. Analytical expressions of mode shapes, natural frequencies and damping factors are used to obtain the modal basis of each string while body modal parameters are extracted from mobility measured at the instrument bridge. In order to compute string time responses to an excitation force, a finite difference scheme is used to discretize and integrate numerically string and body modal equations in time. By imposing displacement continuity at the points where strings and body are connected, an expression to calculate the set of unknown coupling forces at each instant is derived, which allows the computation of string responses. This strategy of solution has been previously adopted for the violin [?] and piano [?].

The method is applied for the sound synthesis of viola caipira. The main vibroacoustic phenomena occurring in the instrument such as string/string collisions, string sympathetic vibrations and beating tones are discussed and reproduced by means of a fully coupled model including non-planar vibrations of 10 strings coupled to the body through the bridge. 


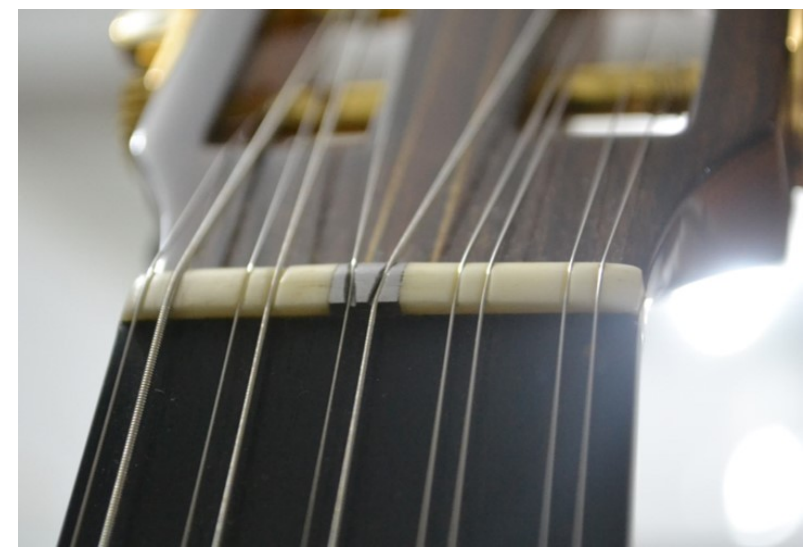

Figure 4.1: String arrangement in the viola caipira: five courses of double strings. From right to left, the strings are numbered from 1 to 10 and pairs from 1 to 5 .

The viola caipira is originally played in numerous cultural manifestations associated to ludic-religious practices in the Central South region of Brazil. The instrument is closely related with the musical genres called caipira and sertanejo, and in the recent decades has significantly interacted with other genres of the classical and popular modern musics. The viola caipira has, in general, 10 metal strings arranged in five courses of double strings and the thinnest string (string 6 referred in Figure ??) is located in the middle. The first two courses have identical strings tuned in unison, while the other three have strings with different diameters tuned in an octave. Strings 5, 7 and 9 are composed of a metal core covered in wound metal (wound strings), while the others are composed of a single metal wire (flat strings). The body shape is similar to those of classical guitars although with a smaller size and slightly narrower waist. It is estimated that there are approximately twenty ways to tune the instrument [?]. Some of these tunings are rarely used, while others are widespread in different regions of Brazil.

This paper is organized as follows: in Section 2 is presented an experimental study of the viola caipira pluck using a high speed camera. A collision model used to calculate impact forces between strings is described in Section ??. In Section ??, a modal-based model of the fully coupled system including 10 strings with non-planar motions interacting with the instrument body is presented. The determination of the model parameters and a set of simulations reproducing physical aspects of the viola caipira are finally obtained and discussed in Section ??.

\subsection{Experimental observations}

The objective of this section is to identify experimentally string/string collisions in the viola caipira by means of a high speed camera. Experimental tests are performed on a single instrument (Rozini brand, Ponteio Profissional model).

\subsubsection{High speed camera setup}

In order to analyse the motion of strings during and after a pluck, an experimental observation was carried out using a high speed camera Photron, model FASTCAM SA-X2, which provided an imaging performance of $1024 \times 768$ pixels of resolution at the recording rate of 5000 frames per second. To facilitate the observation of the strings motions, the guitar was placed horizontally on a flat surface with its back plate in contact with a piece of foam, as shown in Figure ??. A mirror attached to a stand was 
also positioned next to the instrument to capture images from a different angle than that captured directly by the camera. The fourth pair (strings 7 and 8) was then plucked in the downward direction of strings using the thumbnail.

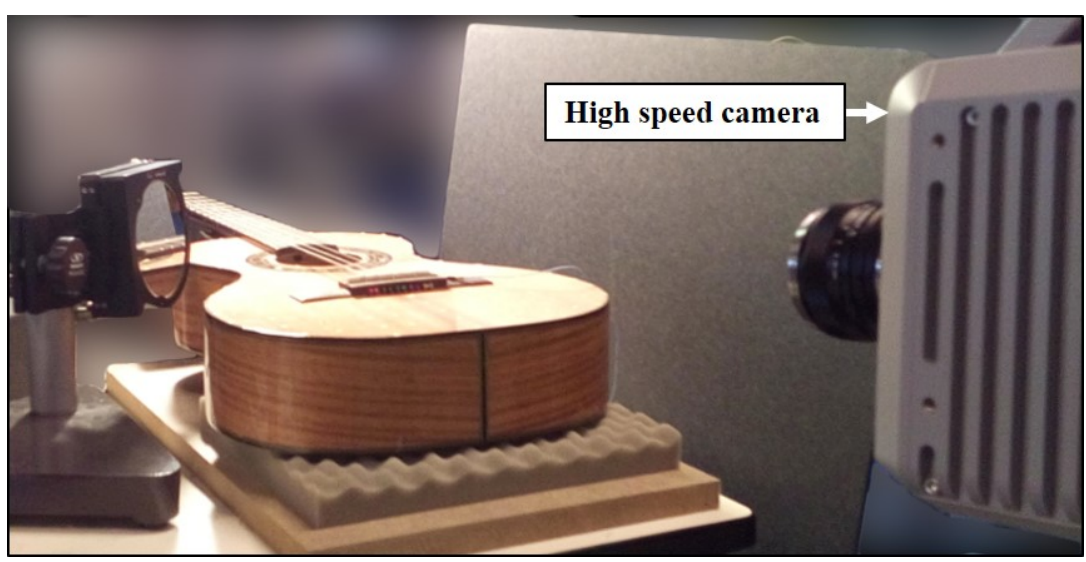

Figure 4.2: Experimental setup for motion analysis of the viola caipira strings using a high speed camera.

\subsubsection{Identification of string/string collisions}

As shown in the series of snapshots in Figures ??a and ??b, strings 8 and 7 are plucked successively and rapidly, within a time interval $\Delta t_{\text {pluck }}=14 \mathrm{~ms}$ for the presented measure. This single measure of the $\Delta t_{\text {pluck }}$ allow us to understand the phenomenon and provides a realistic value to initialize the algorithm for sound synthesis presented in Section ??. However, it is worth highlighting that the value of $\Delta t_{\text {pluck }}$ may be affected directly by factors such as the spacing between strings, string diameters, instrument tuning and pluck direction. Figures ??c to ??e show that the strings collided successively three times, but not only, after the string 7 is plucked (second pluck), within an interval of $3 \mathrm{~ms}$. This phenomenon of string/string collisions is a remarkable specificity of the viola caipira and may strongly influence the sound produced. This issue is addressed in Section ?? by means of sound simulations.

Not shown here, other plucks have been observed. Different plucking conditions were used and different strings were excited. It was found that the occurrence of collisions varies from pair to pair and depends on the string spacing, excitation force and plucking direction. Collisions were observed only in cases where pairs 3,4 and 5 were plucked in a direction predominantly parallel to the soundboard by applying moderate force. Since the tensions on the strings of pairs 1 and 2 are relative higher, their vibration amplitude is in general smaller considering that the same excitation force is applied. Thus, to induce collisions in pairs 1 and 2, a higher plucking force is required. Even though in a qualitative way, such observations give us reasonable results to conclude that the existence of string/string collisions is associated to characteristics intrinsic to the instrument (string tensions), parameters controlled by the musician (direction and force of the pluck) and parameters adjusted by the instrument maker (adjustment of string spacing). 

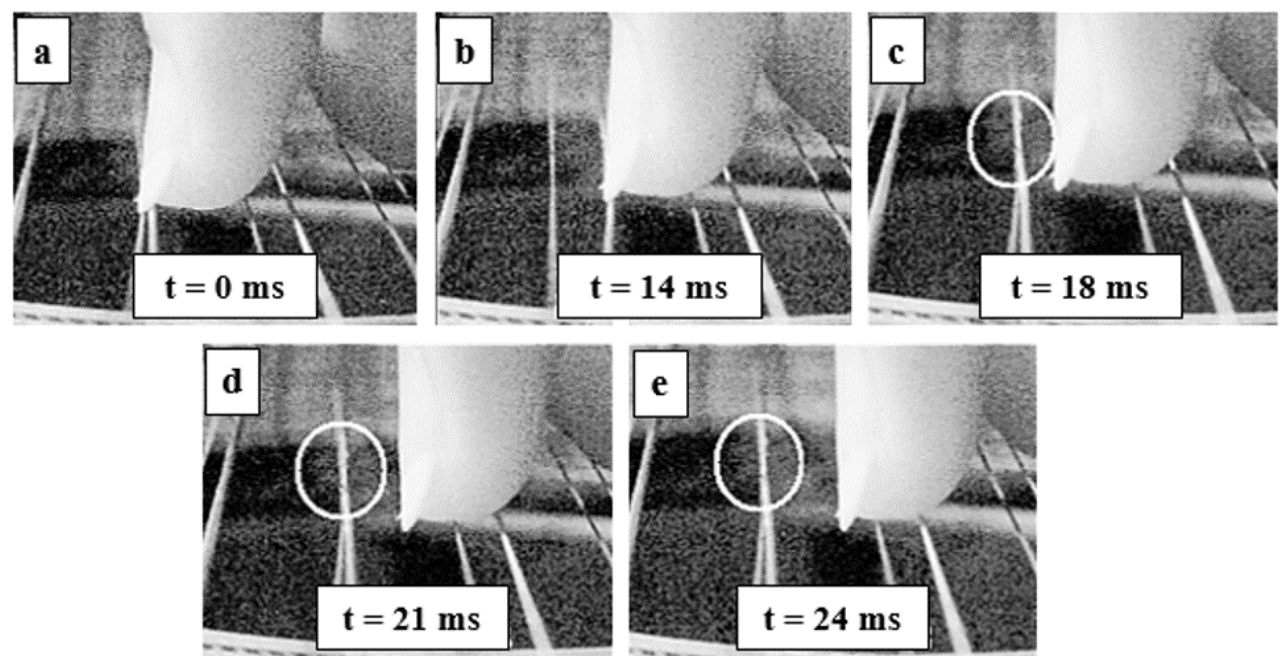

Figure 4.3: Series of snapshots captured during a viola caipira pluck with the thumbnail. (a) First pluck at string 8 (D4); (b) second pluck at string 7 (D3) after 14 ms. Three successive collisions between the two strings are shown in (c), (d) and (e).

\subsection{String/string collisions modelling}

The experimental results presented in Section ?? revealed the occurrence of string/string collisions in the viola caipira and such phenomena are expected to play a relevant role on the sound produced. To investigate numerically this issue, a string/string collision model is first described in this section.

\section{A. Kinematics of two colliding strings}

Based on the above-presented experimental observations, it is assumed that collisions occur only between strings located in the same pair. For the sake of simplicity, a single pair of strings is considered in the formulation below (strings 1 and 2). Let us consider two co-planar cross-sections with radii $r_{1}$ and $r_{2}$, as depicted in Figure ??, whose coordinates $\left(Y^{1}(x, t), Z^{1}(x, t)\right)$ and $\left(Y^{2}(x, t), Z^{2}(x, t)\right)$ are given by

$$
Y^{(s)}(x, t)=y_{c}^{(s)}+y^{(s)}(x, t) \quad \text { and } \quad Z^{(s)}(x, t)=z_{c}^{(s)}+z^{(s)}(x, t),
$$

with $s=1,2$. Note that for a the for given string $s$ at rest, the coordinate of a generic cross-section is given by $\left(y_{c}^{(s)}, z_{c}^{(s)}\right)$. This problem is similar to those of two-ball collisions (see for example [?]). As shown in Figure ??, the distance between the centroids of the cross-sections writes

$$
r(x, t)=\sqrt{\left(Y^{2}(x, t)-Y^{1}(x, t)\right)^{2}+\left(Z^{2}(x, t)-Z^{1}(x, t)\right)^{2}}
$$

and the angle $\gamma(x, t)$ is given by

$$
\gamma(x, t)=\arctan \left(\frac{Z^{2}(x, t)-Z^{1}(x, t)}{Y^{2}(x, t)-Y^{1}(x, t)}\right) .
$$




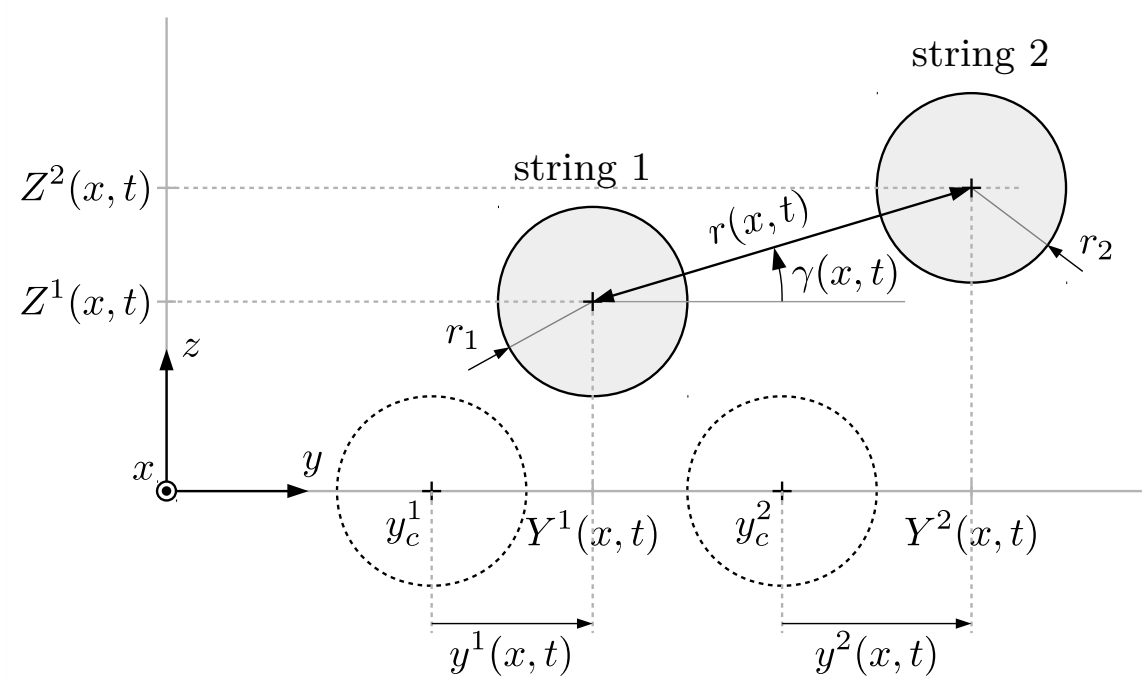

(a)

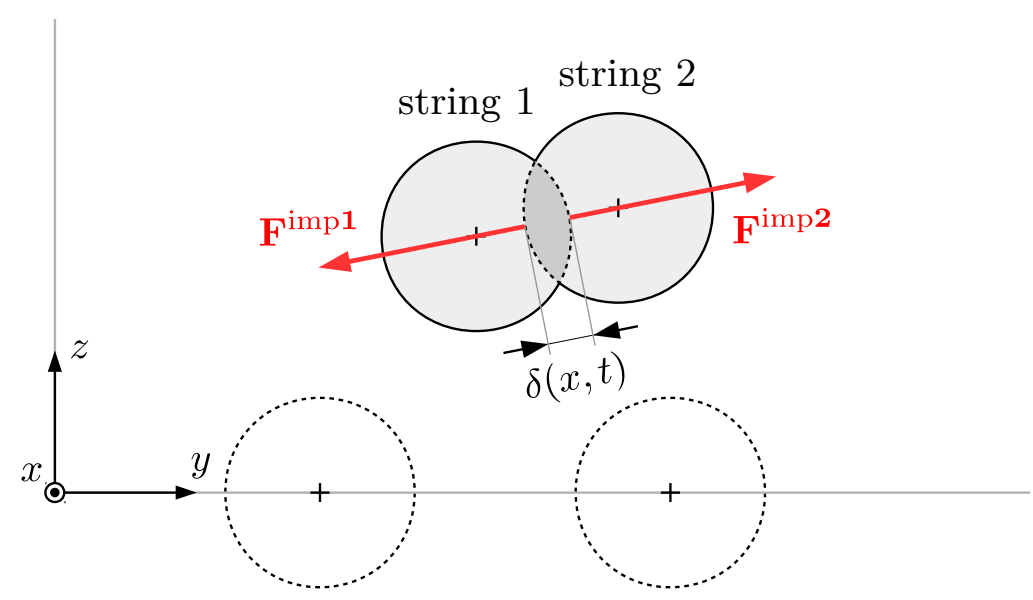

(b)

Figure 4.4: (a) Scheme of coordinates for two cross-sections parallel to the $z y$-plane; (b) Scheme of impact forces at two colliding cross-sections.

\section{B. Collision model}

Impact is defined as the interaction between two colliding bodies throughout a short-time collision event, during which large resultant pairs of action-reaction forces act in opposite directions over the contact area of the colliding bodies [?, ?]. Such phenomenon is also characterized by the occurrence of a compression phase followed by a restitution phase. During the former phase the two bodies come into contact and press against each other. In the latter phase the bodies move away from one another while remaining in contact [?].

Figure ?? depicts the contact between two colliding coplanar cross-sections located at a generic point 
$x$, where $\delta(x, t)$ is the resultant collision indentation. A pointwise string/string contact is assumed. The vectors $\mathbf{F}^{\text {imp1 } 1}(x, t)=\left(F^{i m p 1 y}, F^{i m p 1 z}\right)$ and $\mathbf{F}^{\text {imp2 }}(x, t)=\left(F^{i m p 2 y}, F^{\text {imp2z }}\right)$ represent the pair of action-reaction impact forces acting at $x$, where a frictionless contact between the cross-sections occurs. The non-linear model for elastic collisions proposed by Hunt and Crossley [?] is used. It incorporates a hysterical damping term to the classical Hertz model [?]. The energy loss during the impact occurs through heat dissipation caused by internal damping mechanisms intrinsic to the colliding body materials. Since body deformation is assumed to occur in the elastic range of material properties, the body shape remains undeformed after the contact period. The vectors of impact forces $\mathbf{F}^{\mathbf{i m p 1}}(x, t)$ and $\mathbf{F}^{\mathrm{imp}}(x, t)$ are opposite with identical magnitudes given by

$$
\left|\mathbf{F}^{\mathbf{i m p}(\mathbf{s})}(x, t)\right|=K_{c} \delta^{p}(x, t)+\lambda_{d} \delta^{p}(x, t) \dot{\delta}(x, t),
$$

where $\delta(x, t)=\max \left\{0, r(x, t)-\left(r_{1}+r_{2}\right)\right\}$, with $s=1,2$, where $K_{c}$ is the contact stiffness, $p$ is the compliance exponent, $\lambda_{d}$ is the damping coefficient, $\dot{\delta}(x, t)$ is the indentation velocity.

The $y$ and $z$ components of the impact force vector $\mathbf{F}^{\text {imply }}(x, t)$ are computed as follows:

$$
F^{\text {imp1y }}=-\left|\mathbf{F}^{\text {imp1 }}(x, t)\right| \cos (\gamma(x, t))
$$

and

$$
F^{i m p 1 z}=-\left|\mathbf{F}^{\text {imp1 }}(x, t)\right| \sin (\gamma(x, t)) .
$$

The y and z components of $\mathbf{F}^{\text {imp2 }}(x, t)$ are then given by $F^{\text {imp2y }}=-F^{\text {imp1y }}$ and $F^{\text {imp2z }}=-F^{\text {imp1z }}$. Since the impact forces are calculated along the entire string length, a distribution of impact forces, given in $N / m$, is considered in Section ??.

\subsection{Fully coupled system modelling}

Let us consider a set of 10 stiff strings coupled to the body. For a given string $s$, a small-amplitude vibration is assumed and the following properties are assigned: length $L^{(s)}$, mass per unit length $\mu^{(s)}$, tension $T^{(s)}$ and bending stiffness $B^{(s)}$. The string is simply supported at one end located at $x^{(s)}=0$, and attached to the body through the bridge so that it is allowed to move at the other end located at $x_{c}^{(s)}=L^{(s)}$. Body and string displacements are set to be identical at $x_{c}^{(s)}=L^{(s)}$ in such a way that both structures are coupled. The string transverse motion is expressed as the sum of the components $y^{(s)}(x, t)$ and $z^{(s)}(x, t)$, oriented normal and parallel to the soundboard plane, respectively (see Figure ??). Accordingly, the transverse body motion at the $s^{\text {th }}$ string coupling point $\mathbf{p}_{\mathbf{c}}^{(\mathbf{s})}=\left(x_{c}^{(s)}, y_{c}^{(s)}, z_{c}^{(s)}\right)$ is described by the components $y^{b}\left(\mathbf{p}_{\mathbf{c}}^{(\mathbf{s})}, t\right)$ and $z^{b}\left(\mathbf{p}_{\mathbf{c}}^{(\mathbf{s})}, t\right)$. Axial and torsional string motions are neglected since they are ineffective in exciting the instrument body. Geometrical non-linearities intrinsic to the strings are also neglected.

\subsubsection{Modal formulation}

\section{A. String kinematics}

For a given string $s$, the displacement in each of the two orthogonal polarizations is described as the sum of $N_{(s)}$ modes associated to the string with simply supported ends to which an interface mode is added, whose shape

$$
\phi_{0}^{(s)}(x)=\left(\frac{x}{L^{(s)}}\right)
$$




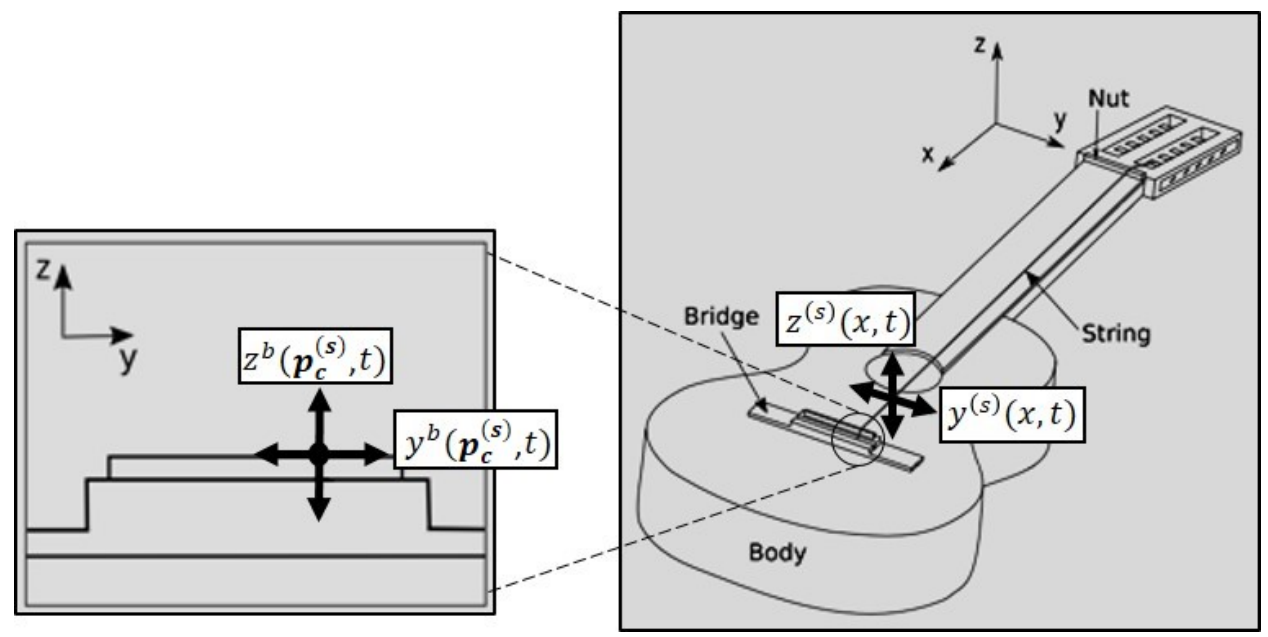

Figure 4.5: Scheme representing the decomposition of string and body non-planar transverse motions into components normal and parallel to the soundboard.

corresponds to the static response of the string when it is simply supported at $x^{(s)}=0$ and loaded at the end attached to the body, $x_{c}^{(s)}=L^{(s)}$. Accordingly, the transverse displacements $y^{(s)}(x, t)$ and $z^{(s)}(x, t)$ write

$$
y^{(s)}(x, t)=a_{0}^{(s) y}(t) \phi_{0}^{(s)}(x)+\sum_{j=1}^{N_{(s)}} a_{j}^{(s) y}(t) \phi_{j}^{(s) y}(x)
$$

and

$$
z^{(s)}(x, t)=a_{0}^{(s) z}(t) \phi_{0}^{(s)}(x)+\sum_{j=1}^{N_{(s)}} a_{j}^{(s) z}(t) \phi_{j}^{(s) z}(x),
$$

where $\phi_{j}^{(s) y}(x)$ and $\phi_{j}^{(s) z}(x)$ are the $j^{t h}$ mode shapes in both string polarizations, $a_{j}^{(s) y}(t)$ and $a_{j}^{(s) z}(t)$ are the corresponding modal amplitudes, and $a_{0}^{(s) y}(t)$ and $a_{0}^{(s) z}(t)$ are the modal amplitudes associated to the interface mode $\phi_{0}^{(s)}(x)$. The mode shapes associated to the string with both simply supported ends are given by

$$
\phi_{j}^{(s) y}(x)=\phi_{j}^{(s) z}(x)=\sin \left(\frac{j \pi x}{L^{(s)}}\right) .
$$

The $j^{\text {th }}$ modal angular frequency taking into account the string inharmonicity is given by $\omega_{j}^{(s)}=$ $\frac{\pi j c^{(s)}}{L^{(s)}} \sqrt{1+j^{2}\left(\frac{B^{(s)} \pi^{2}}{T^{(s)}\left(L^{(s)}\right)^{2}}\right)}$, where $c^{(s)}=\sqrt{\frac{T^{(s)}}{\mu^{(s)}}}$ is the wave velocity of the ideal string. For further formulation, it is convenient to define the generic vector of string mode shapes

$$
\boldsymbol{\phi}^{(s)(w)}(x)=\left(\phi_{0}^{(s)(w)}(x), \phi_{1}^{(s)(w)}(x), \ldots, \phi_{N_{(s)}}^{(s)(w)}(x)\right),
$$

where $s=1, \ldots, 10$ and $w$ stands for $y$ or $z$. 


\section{B. Body kinematics}

Accordingly, the body transverse displacements at the $s^{\text {th }}$ string/body coupling point $\mathbf{p}_{\mathbf{c}}^{(\mathbf{s})}$, in both polarizations, are described as the sum of $N_{b}$ body modes:

$$
y^{b}\left(\mathbf{p}_{\mathbf{c}}^{(\mathbf{s})}, t\right)=\sum_{k=1}^{N_{b}} b_{k}(t) \phi_{k}^{b y}\left(\mathbf{p}_{\mathbf{c}}^{(\mathbf{s})}\right)
$$

and

$$
z^{b}\left(\mathbf{p}_{\mathbf{c}}^{(\mathbf{s})}, t\right)=\sum_{k=1}^{N_{b}} b_{k}(t) \phi_{k}^{b z}\left(\mathbf{p}_{\mathbf{c}}^{(\mathbf{s})}\right),
$$

where $\phi_{k}^{b y}\left(\mathbf{p}_{\mathbf{c}}^{(\mathbf{s})}\right)$ and $\phi_{k}^{b z}\left(\mathbf{p}_{\mathbf{c}}^{(\mathbf{s})}\right)$ are the $k^{\text {th }}$ body mode shapes at $\mathbf{p}_{\mathbf{c}}^{(\mathbf{s})}$ in both polarizations, and $b_{k}(t)$ is the corresponding modal amplitude. It is also convenient to define the generic vector of body mode shapes

$$
\boldsymbol{\phi}^{b(w)}\left(\mathbf{p}_{\mathbf{c}}^{(\mathbf{s})}\right)=\left(\phi_{1}^{b(w)}\left(\mathbf{p}_{\mathbf{c}}^{(\mathbf{s})}\right), \ldots, \phi_{N_{b}}^{b(w)}\left(\mathbf{p}_{\mathbf{c}}^{(\mathbf{s})}\right)\right)
$$

where $s=1, \ldots, 10$, and $w$ stand for $y$ or $z$.

\section{String dynamics}

Within a modal framework, the motion equations of the 10 strings for a forced response can be formulated as a set of $\sum_{s=1}^{10} 2\left(N_{(s)}+1\right)$ secondary-order ordinary differential equations (ODEs) and written in the following matrix form:

$$
\begin{aligned}
& \underbrace{\left[\begin{array}{ccc}
\mathbf{M}^{\mathbf{1}} & & (\mathbf{0}) \\
& \ddots & \\
(\mathbf{( 0 )} & & \mathbf{M}^{\mathbf{1 0}}
\end{array}\right]}_{\mathbf{M}^{\mathbf{s}}} \underbrace{\left[\begin{array}{c}
\ddot{\mathbf{a}}^{\mathbf{1}}(t) \\
\vdots \\
\ddot{\mathbf{a}}^{\mathbf{1 0}}(t)
\end{array}\right]}_{\ddot{\mathbf{a}}(t)}+\underbrace{\left[\begin{array}{ccc}
\mathbf{C}^{\mathbf{1}} & & \mathbf{( 0 )} \\
& \ddots & \\
(\mathbf{0}) & & \mathbf{C}^{\mathbf{1 0}}
\end{array}\right]}_{\mathbf{C}^{\mathbf{s}}} \underbrace{\left[\begin{array}{c}
\dot{\mathbf{a}}^{\mathbf{1}}(t) \\
\vdots \\
\dot{\mathbf{a}}^{\mathbf{1 0}}(t)
\end{array}\right]}_{\dot{\mathbf{a}}(t)}+\ldots \\
& \ldots+\underbrace{\left[\begin{array}{ccc}
\mathbf{K}^{\mathbf{1}} & & (\mathbf{0}) \\
& \ddots & \\
(\mathbf{0}) & & \mathbf{K}^{\mathbf{1 0}}
\end{array}\right]}_{\mathbf{K}^{\mathbf{s}}} \underbrace{\left[\begin{array}{c}
\mathbf{a}^{\mathbf{1}}(t) \\
\vdots \\
\mathbf{a}^{\mathbf{1 0}}(t)
\end{array}\right]}_{\mathbf{a}(t)}=\underbrace{\left[\begin{array}{c}
\mathbf{f}^{\mathbf{1}}(t) \\
\vdots \\
\mathbf{f}^{\mathbf{1 0}}(t)
\end{array}\right]}_{\mathbf{f}^{\mathbf{s}}(t)},
\end{aligned}
$$

where $\mathbf{M}^{\mathbf{s}}, \mathbf{C}^{\mathbf{s}}$ and $\mathbf{K}^{\mathbf{s}}$ are respectively the modal mass, modal damping and modal stiffness matrices. The partitioned column vectors $\mathbf{a}$ and $\mathbf{f}$ s represent the modal coordinates and the associated modal forces of the 10 strings in the two polarizations. For a given string $s$, the corresponding subvectors setting up a and $\mathbf{f}^{\mathrm{s}}$ are respectively given by

$$
\mathbf{a}^{(s)}(t)=\left(a_{0}^{(s) y}(t), \ldots, a_{N_{(s)}}^{(s) y}, a_{0}^{(s) z}(t), \ldots, a_{N_{(s)}}^{(s) z}(t)\right)^{T}
$$

and

$$
\mathbf{f}^{(s)}(t)=\left(f_{0}^{(s) y}(t), \ldots, f_{N_{(s)}}^{(s) y}, f_{0}^{(s) z}(t), \ldots, f_{N_{(s)}}^{(s) z}(t)\right)^{T}
$$


All the matrices in Equation ?? are block diagonal matrices set up by submatrices containing the string modal properties of each string. For a given string $s(s=1, \ldots, 10)$,

$$
\mathbf{M}^{(s)}=\left[\begin{array}{cc}
\mathbf{M}^{(s) y} & \mathbf{0} \\
\mathbf{0} & \mathbf{M}^{(s) z}
\end{array}\right], \quad \mathbf{C}^{(s)}=\left[\begin{array}{cc}
\mathbf{C}^{(s) y} & \mathbf{0} \\
\mathbf{0} & \mathbf{C}^{(s) z}
\end{array}\right] \text { and } \quad \mathbf{K}^{(s)}=\left[\begin{array}{cc}
\mathbf{K}^{(s) y} & \mathbf{0} \\
\mathbf{0} & \mathbf{K}^{(s) z}
\end{array}\right]
$$

where, for $w=y, z$,

$$
\begin{gathered}
\mathbf{M}^{(s)(w)}=\left[\begin{array}{ccccc}
\frac{\mu^{(s)} L^{(s)}}{3} & \frac{L^{(s)} \mu^{(s)}}{1 \pi} & \frac{L^{(s)} \mu^{(s)}}{2 \pi} & \ldots & \frac{L^{(s)} \mu_{(s)}^{(s)}}{N_{(s)}} \\
\frac{L^{(s)} \mu^{(s)}}{1 \pi} & \frac{\mu^{(s)} L^{(s)}}{2} & & (\mathbf{0}) \\
\frac{L^{(s)} \mu^{(s)}}{2 \pi} & & \frac{\mu^{(s)} L^{(s)}}{2} & & \\
\vdots & (\mathbf{0}) & & \ddots & \\
\frac{L^{(s)} \mu^{(s)}}{N_{(s)} \pi} & & & \frac{\mu^{(s)} L^{(s)}}{2}
\end{array}\right], \\
\mathbf{K}^{(s)(w)}=\operatorname{diag}\left(\frac{T^{(s)}}{L^{(s)}}, \frac{T^{(s)} \pi^{2}}{2 L^{(s)}}+\frac{B^{(s)} \pi^{4}}{2\left(L^{(s)}\right)^{3}}, \cdots, \frac{N_{(s)}^{2} T^{(s)} \pi^{2}}{2 L^{(s)}}+\frac{N_{(s)}^{4} B^{(s)} \pi^{4}}{2\left(L^{(s)}\right)^{3}}\right),
\end{gathered}
$$

and

$$
\mathbf{C}^{(s)(w)}=\operatorname{diag}\left(0, \frac{\pi \sqrt{T^{(s)} \mu^{(s)}}}{2 Q_{1}^{(s)}}, \cdots, \frac{\pi \sqrt{T^{(s)} \mu^{(s)}}}{2 Q_{N_{(s)}}^{(s)}}\right),
$$

where $Q_{j}^{(s)}$ is the Q-factor associated to the $j^{\text {th }}$ string mode. In ??, the computation of $\mathbf{M}^{(s)}$ and $\mathbf{K}^{(s)}$ is presented in details. A simplified way to include a viscous damping model consists in assuming the damping matrix is diagonal for both strings and body. This assumption has no real physical background but is consistent with the assumption that the structure is lightly damped (see for example [?], Chapter 3). The string damping model used to calculate $Q_{j}^{(s)}$ is described in ??.

Let us consider that the external physical forces acting on a given string $s$ are:

- The excitation force $\mathbf{F}^{\mathrm{e}(\mathbf{s})}(t)=\left(F^{e(s) y}, F^{e(s) z}\right)$ at the point $x_{e}^{(s)}$, applied in an angle $\theta^{(s)}$ from the positive $y$-axis so that $F^{e(s) y}=\left|\mathbf{F}^{\text {(s) }}(t)\right| \cos \theta^{(s)}$ and $F^{e(s) z}=\left|\mathbf{F}^{\mathbf{e}(\mathbf{s})}(t)\right| \sin \theta^{(s)}$;

- The coupling force $\mathbf{F}^{\mathbf{c}(\mathbf{s})}(t)=\left(F^{c(s) y}, F^{c(s) z}\right)$ at the point $x_{c}^{(s)}$ resulting from the string/body interaction;

- The distributed impact forces acting on the string given by $\mathbf{F}^{\text {imp(s) }}(x, t)=\left(F^{\operatorname{imp}(s) y}(x, t), F^{\operatorname{imp}(s) z}(x, t)\right)$.

The distribution of external forces $\mathbf{F}^{(s)}(x, t)=\left(F^{(s) y}(x, t), F^{(s) z}(x, t)\right)$ acting on the string then writes

$$
\mathbf{F}^{(\mathbf{s})}(x, t)=\mathbf{F}^{\mathrm{e}(\mathbf{s})}(t) \delta\left(x-x_{e}^{(s)}\right)+\mathbf{F}^{\mathrm{c}(\mathbf{s})}(t) \delta\left(x-x_{c}^{(s)}\right)+\mathbf{F}^{\mathrm{imp}(\mathrm{s})}(x, t)
$$

so that the string forces associated to the $j^{\text {th }}$ mode $\left(j=0, \ldots, N_{(s)}\right)$, in both polarizations, are then calculated by projecting the components of $\mathbf{F}^{(\mathbf{s})}(x, t)$ on the corresponding string mode shapes:

$$
f_{j}^{(s) y}(t)=\int_{0}^{L^{(s)}} F^{(s) y}(x, t) \phi_{j}^{(s) y}(x) d x \quad \text { and } \quad f_{j}^{(s) z}(t)=\int_{0}^{L^{(s)}} F^{(s) z}(x, t) \phi_{j}^{(s) z}(x) d x .
$$

The string is discretized into a set of $M(s)$ points using a space step $\Delta x$ so that $M(s)=\frac{L^{(s)}}{\Delta x}$. This approach allows to compute separately collision forces at each point $x_{n}=n \Delta x(n=1, \ldots, M(s))$ in case of string/string contact by using Equation ??. An approximation of Equation ?? can be finally obtained:

$$
\mathbf{f}^{\mathbf{s}}=\left(\boldsymbol{\Phi}_{\mathbf{e}}^{\mathbf{s}}\right)^{T} \mathbf{F}^{\mathbf{e}}-\left(\boldsymbol{\Phi}_{\mathbf{c}}^{\mathbf{s}}\right)^{T} \mathbf{F}^{\mathbf{c}}+\left(\boldsymbol{\Phi}_{\text {imp }}^{\mathbf{s}}\right)^{T} \mathbf{F}^{\mathrm{imp}} \Delta x,
$$


where

$$
\begin{aligned}
& \mathbf{F}^{\mathbf{c}}=\left(F^{c 1 y}, F^{c 1 z}, \ldots, F^{c 10 y}, F^{c 10 z}\right)^{T}, \\
& \mathbf{F}^{\mathbf{e}}=\left(F^{e 1 y}, F^{e 1 z}, \ldots, F^{e 10 y}, F^{e 10 z}\right)^{T},
\end{aligned}
$$

and

$$
\mathbf{F}^{\text {imp }}=\left(F^{i m p 1 y}, F^{i m p 1 z}, \ldots, F^{i m p 10 y}, F^{i m p 10 z}\right)^{T} .
$$

Matrices $\boldsymbol{\Phi}_{\mathrm{e}}^{\mathrm{s}}, \boldsymbol{\Phi}_{\mathbf{c}}^{\mathbf{s}}$ and $\boldsymbol{\Phi}_{\mathrm{imp}}^{\mathrm{s}}$ are described in ??

\section{E. Body dynamics}

Similarly to the strings, the equations describing the body motion are also written within a modal framework and formulated in the matrix form as the set of $N_{b}$ secondary-order ODEs

$$
\mathbf{M}^{\mathbf{b}} \ddot{\mathbf{b}}(t)+\mathbf{C}^{\mathbf{b}} \dot{\mathbf{b}}(t)+\mathbf{K}^{\mathbf{b}} \mathbf{b}(t)=\mathbf{f}^{\mathbf{b}}(t),
$$

where $\mathbf{M}^{\mathbf{b}}, \mathbf{C}^{\mathbf{b}}$ and $\mathbf{K}^{\mathbf{b}}$ are respectively the mass, damping and stiffness modal matrices, which are given by

$$
\begin{gathered}
\mathbf{M}^{\mathbf{b}}=\operatorname{diag}\left(m_{1}^{b}, \ldots, m_{N_{b}}^{b}\right), \\
\mathbf{C}^{\mathbf{b}}=\operatorname{diag}\left(2 m_{1}^{b} \omega_{1}^{b} \zeta_{1}^{b}, \ldots, 2 m_{N_{b}}^{b} \omega_{N_{b}}^{b} \zeta_{N_{b}}^{b}\right)
\end{gathered}
$$

and

$$
\mathbf{K}^{\mathbf{b}}=\operatorname{diag}\left(m_{1}^{b}\left(\omega_{1}^{b}\right)^{2}, \ldots, m_{N_{b}}^{b}\left(\omega_{N_{b}}^{b}\right)^{2}\right),
$$

where $m_{k}^{b}, \zeta_{k}^{b}$ and $\omega_{k}^{b}\left(k=1, \ldots, N_{b}\right)$ are respectively the mass, damping ratio and angular frequency associated to the $k^{t h}$ body mode. The column vectors $\mathbf{b}$ and $\mathbf{f}^{\mathbf{b}}$ represent respectively the modal coordinates and the associated modal forces of the body given by

$$
\mathbf{b}(t)=\left(b_{1}(t), \ldots, b_{N_{b}}(t)\right)^{T}
$$

and

$$
\mathbf{f}^{\mathbf{b}}(t)=\left(f_{1}^{b}(t), \ldots, f_{N_{b}}^{b}(t)\right)^{T} .
$$

The distribution of external forces acting at a generic point $\mathbf{p}=(x, y, z)$ on the body is given by $\mathbf{F}^{\mathbf{b}}(\mathbf{p}, t)=\left(F^{b y}(\mathbf{p}, t), F^{b z}(\mathbf{p}, t)\right)$, with $\mathbf{p} \in \Omega$, where $\Omega$ represents the structural body domain. Assuming a continuous representation of the body structure, the modal force associated to the $k^{\text {th }}$ body mode is given by the modal projection

$$
f_{k}^{b}(t)=\int_{\Omega} F^{b y}(\mathbf{p}, t) \phi_{k}^{b y}(\mathbf{p}) d s+\int_{\Omega} F^{b z}(\mathbf{p}, t) \phi_{k}^{b z}(\mathbf{p}) d s,
$$

Let us consider that $\mathbf{F}^{\mathbf{b}}(\mathbf{p}, t)$ is only composed of the set of forces resulting from strings/body interactions at the coupling points $\mathbf{p}_{\mathbf{c}}^{(\mathbf{s})}$, with $s=1, \ldots, 10$. According to the action and reaction principle, the forces exerted by the strings on the body are equal in magnitude and opposite in direction to the forces exerted by the body on the strings so that

$$
F^{b y}(\mathbf{p}, t)=\sum_{s=1}^{10} F^{c(s) y}(t) \delta^{2}\left(\mathbf{p}-\mathbf{p}_{\mathbf{c}}^{(\mathbf{s})}\right) \quad \text { and } \quad F^{b z}(\mathbf{p}, t)=\sum_{s=1}^{10} F^{c(s) z}(t) \delta^{2}\left(\mathbf{p}-\mathbf{p}_{\mathbf{c}}^{(\mathbf{s})}\right) .
$$


Equation ?? then are rewritten as the sum of contributions from the coupling forces at the 10 string/body attachment points as follows

$$
\mathbf{f}^{\mathbf{b}}=\left(\boldsymbol{\Phi}_{\mathbf{c}}^{\mathbf{b}}\right)^{T} \mathbf{F}^{\mathbf{c}}
$$

where

$$
\boldsymbol{\Phi}_{\mathbf{c}}^{\mathbf{b}}=\left[\begin{array}{c}
\boldsymbol{\phi}^{b y}\left(p_{c}^{1}\right) \\
\boldsymbol{\phi}^{b z}\left(p_{c}^{1}\right) \\
\vdots \\
\boldsymbol{\phi}^{b y}\left(p_{c}^{10}\right) \\
\boldsymbol{\phi}^{b z}\left(p_{c}^{10}\right)
\end{array}\right] .
$$

\subsection{Numerical simulations}

\subsubsection{Finite differences scheme}

The first step to solve equations ?? and ?? numerically is to discretize them in time domain. Let us consider the time series $w_{i}$ ( $w$ stand for $a, b, F^{c}, F^{e}$ and $F^{i m p}$ ), which represents an approximation to $w\left(t_{i}\right)$, where $t_{i}=i \Delta t$, for the time step $i$, so that $\Delta t=t_{i+1}-t_{i}$. The sampling frequency $f_{s}$ is the number of samples per second in the synthesized signal and is given by $f_{s}=\frac{1}{\Delta t}$.

\section{A. Recurrence equations}

Approximations of the first and second derivatives in Equations ?? and ?? are obtained using centred finite differences and recurrence equations associated to strings and body modal displacements are written as follows:

$$
\begin{aligned}
& \mathbf{a}_{i+1}=\mathbf{A} \boldsymbol{\alpha}-\mathbf{G}^{\mathbf{s}} \mathbf{F}_{i}^{\mathbf{c}}, \\
& \mathbf{b}_{i+1}=\mathbf{B} \boldsymbol{\beta}-\mathbf{G}^{\mathbf{b}} \mathbf{F}_{i}^{\mathbf{c}},
\end{aligned}
$$

where $\mathbf{A}, \boldsymbol{\alpha}, \mathbf{G}^{\mathbf{s}}, \mathbf{B}, \boldsymbol{\beta}$ and $\mathbf{G}^{\mathbf{b}}$ are as given in Table $? ?$ in $? ?$.

\section{B. Coupling forces computation}

To obtain an expression to compute the vector $\mathbf{F}_{i}^{\mathbf{c}}$, whose components are unknown, the displacement continuity at the $s^{\text {th }}$ string/body coupling point $(s=1, \ldots, 10)$ is imposed at $t_{i+1}$ :

$$
z^{(s)}\left(x_{c}^{(s)}, t_{i+1}\right)=z^{b}\left(p_{c}^{(s)}, t_{i+1}\right) \Longrightarrow \boldsymbol{\phi}^{(s)}\left(x_{c}^{(s)}\right) \mathbf{a}_{i+1}^{(s)}=\boldsymbol{\phi}^{\mathbf{b}}\left(p_{c}^{(s)}\right) \mathbf{b}_{i+1}
$$

Substituting Equations ?? and ?? into Equation ??, one may obtain

$$
\mathbf{F}_{i}^{\mathbf{c}}=\left[\mathbf{G}^{\mathbf{s}}+\mathbf{G}^{\mathbf{b}}\right]^{-1}\left[\left(\boldsymbol{\Phi}_{\mathbf{c}}^{\mathbf{s}} \mathbf{A} \boldsymbol{\alpha}\right)-\left(\Phi_{\mathbf{c}}^{\mathbf{b}} \mathbf{B} \boldsymbol{\beta}\right)\right]
$$

Once the coupling forces are computed using Equation ??, one may compute the string modal displacements $\mathbf{a}_{i+1}$ from Equation ??. The string responses in physical coordinates are finally computed by modal superposition. 


\subsubsection{Model parameters}

\section{A. String parameters}

The Rio Abaixo ("Downriver" in English) tuning is used in experiments and simulations. This is a very popular tuning type widespread in many regions of Brazil. Table ?? shows the open string notes, respective fundamental frequencies, mass per unit length, and position coordinates of each string/body coupling point in the $x y$ plane. Strings have the same length, $L^{(s)}=0.585 \mathrm{~m}$, and bending stiffnesses are given by $B^{(s)}=E^{(s)} I^{(s)}$. For the sake of simplicity, all the strings are assumed parallel: usually the spacing between the viola caipira strings at the nut and saddle are not the same and may vary from 2.5 $\mathrm{mm}$ to $3.5 \mathrm{~mm}$. Strings of the same pair are spaced at $3.5 \mathrm{~mm}$ from each other. Modal frequencies of each string are calculated up to $5000 \mathrm{~Hz}$ and inharmonicity effects are taken into account. The usual value of Young's modulus of the steel, $E^{(s)}=2 \times 10^{11} \mathrm{~Pa}$, is selected for all strings. String modal Qfactors are computed from Equation ??. Usual values for standard temperature and pressure conditions for the following parameters are selected: $\rho_{\text {air }}=1.2 \mathrm{~kg} \cdot \mathrm{m}^{-3}$ and $\eta_{\text {air }}=1.8 \times 10^{-5} \mathrm{~kg} \cdot \mathrm{m}^{-1} \cdot \mathrm{s}^{-1}$. Following Valette [?], the value for the sum of viscoelastic and thermoelastic loss angles is $\delta_{v e / t e}=1 \times 10^{-3}$. Finally, the value corresponding to losses due to the dislocation phenomenon is $Q_{d i s l}=5500$, as obtained in [?].

TABLE 4.1: Open string note considering Rio Abaixo tuning, fundamental frequency, diameter, linear density, diameter, modal truncation order and string/body coupling point coordinates for the 10 strings.

\begin{tabular}{lccccc}
\hline & Pair 1 & Pair 2 & Pair 3 & Pair 4 & Pair 5 \\
\hline String number & 1 & 3 & 5 & 7 & 9 \\
\multirow{4}{*}{ Note } & 2 & 4 & 6 & 8 & 10 \\
& $\mathrm{D} 4$ & $\mathrm{~B} 3$ & $\mathrm{G} 3$ & $\mathrm{D} 3$ & $\mathrm{G} 2$ \\
$f_{0}^{(s)}[\mathrm{Hz}]$ & $\mathrm{D} 4$ & $\mathrm{~B} 3$ & $\mathrm{G} 4$ & $\mathrm{D} 4$ & $\mathrm{G} 3$ \\
& 293.67 & 246.94 & 196.00 & 146.80 & 98.00 \\
$d^{(s)}[\mathrm{mm}]$ & 293.67 & 246.94 & 392.00 & 293.70 & 196.00 \\
$\mu^{(s)}\left[10^{-4} \mathrm{~kg} / \mathrm{m}\right]$ & 0.28 & 0.30 & 0.51 & 0.64 & 0.91 \\
& 0.28 & 0.30 & 0.23 & 0.30 & 0.38 \\
$T^{(s)}[\mathrm{N}]$ & 5.27 & 5.93 & 1.41 & 1.88 & 4.35 \\
\multirow{2}{*}{$N_{(s)}$} & 5.27 & 5.93 & 3.78 & 7.16 & 3.8 \\
$\mathbf{p}_{\mathbf{c}}^{(\mathbf{s})}[\mathrm{mm}]$ & 61.57 & 49.12 & 73.94 & 55.49 & 57.39 \\
& 61.57 & 49.12 & 79.3 & 84.52 & 55.72 \\
& 16 & 19 & 25 & 33 & 50 \\
& $(585,0,0)$ & $(585,12.0,0)$ & $(585,24.0,0)$ & $(585,36.0,0)$ & $(585,48.0,0)$ \\
& $(585,3.5,0)$ & $(585,15.5,0)$ & $(585,27.5,0)$ & $(585,39.5,0)$ & $(585,51.5,0)$ \\
\hline
\end{tabular}

\section{B. Body parameters: Modal analysis at the bridge}

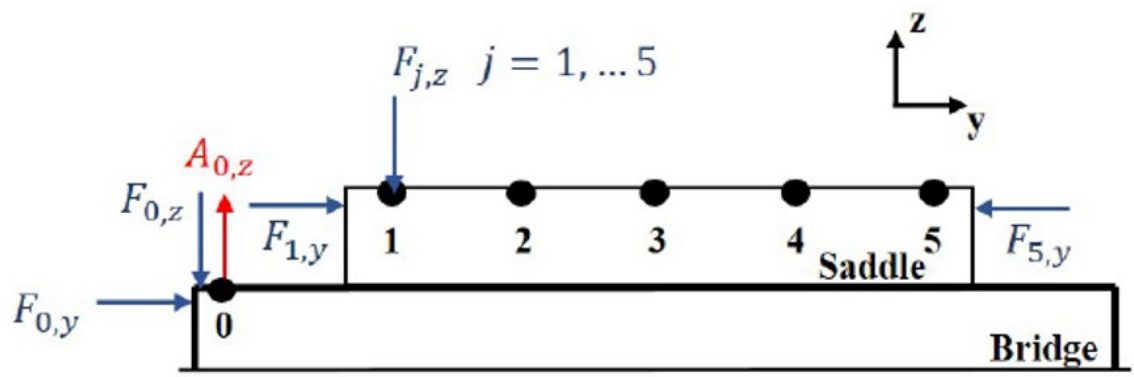


Figure 4.6: Scheme for body modal parameters estimation at 5 string/body coupling points at the bridge saddle using the roving hammer technique.

In order to feed the synthesis model above-presented, the following body modal parameters are required: natural frequencies, modal masses, modal damping ratios and modal shapes in the $y$ and $z$ directions at the string/body coupling points. These parameters are extracted from inertance transfer functions measured at the bridge using the roving hammer technique. Figure ?? illustrates the scheme used for such measurements. Double strings are assumed to be coupled with the body at a single point located at the bridge saddle so that five string/body coupling points (points 1 to 5) are considered for the fully coupled system. From the scheme shown in Figure ??, one may define the inertance functions

$$
H_{j, w}(\omega)=\frac{A_{0, z}(\omega)}{F_{j, w}(\omega)},
$$

where $A_{0, z}$ is the acceleration response measured at the point 0 due to the force $F_{j,(w)}$ applied at the point $j$ in the direction $w(j=0,1, \ldots, 5$, and $w=y, z)$. Since it is not possible to excite coupling points 2 , 3 and 4 in the y direction using the hammer, the following assumptions are made: $H_{2, y}(\omega)=H_{1, y}(\omega)$, $H_{3, y}(\omega)=H_{1, y}(\omega)$ and $H_{4, y}(\omega)=H_{5, y}(\omega)$.

The excitation forces are provided by a miniature impact hammer PCB Piezotronics 086E 80 at points 0 to 5 in the $\mathrm{z}$ direction and points 0,1 and 5 in the y direction; acceleration signals are collected by a lightweight accelerometer PCB Piezotronics 352C $23(0.2 \mathrm{~g})$ mounted on the bridge, fixed at the point 0 shown in Figure ??. Nine inertance transfer functions are obtained from measurements and the other three are obtained from the assumptions described above. Modal parameters are finally estimated using the Polymax method [?] by means of a Multiple-Input, Single-Output (MISO) analysis, which led to 20 body modes between $0 \mathrm{~Hz}$ and $1000 \mathrm{~Hz}$.

\section{Computational parameters}

The plucking point is located at $8.5 \mathrm{~cm}$ from the bridge, i.e. at approximately $1 / 7^{\text {th }}$ of the total string length. A simplified excitation model is used: the force applied to a given string $s$ is a linear ramp whose maximum amplitude is $F_{0}^{(s)}$. The excitation starts at the initial instant $t_{i}^{(s)}$ and ends at the release instant $t_{r}^{(s)}$ so that the excitation force magnitude writes

$$
\left|\mathbf{F}^{\mathrm{e}(\mathrm{s})}(t)\right|=\frac{F_{0}^{(s)}}{\left(t_{r}^{(s)}-t_{i}^{(s)}\right)}\left(t-t_{i}^{(s)}\right)\left(H\left(t-t_{i}^{(s)}\right)-H\left(t-t_{r}^{(s)}\right)\right)
$$

For the simulations presented in this paper $F_{0}^{(s)}=3 \mathrm{~N}$ and $t_{r}^{(s)}-t_{i}^{(s)}=8 \mathrm{~ms}$. Such a force corresponds to a rapid realistic gesture of high amplitude. Impact model parameters are chosen as shown in Table ??. Strings are discretized using a $\Delta x=1 \mathrm{~mm}$. The sampling frequency value $f_{s}$ is selected from the convergence tests presented in Subsection ??.

TABLE 4.2: Impact model parameters

\begin{tabular}{ccc}
\hline$\lambda_{d}\left[N . s / m^{p+2}\right]$ & $K_{c}\left[N / m^{p+1}\right]$ & $p$ \\
\hline $10^{8}$ & $10^{9}$ & 1.5 \\
\hline
\end{tabular}




\subsubsection{Convergence study}

To ensure accurate computations and minimize numerical errors, a small enough $\Delta t$ (or a high enough $f_{s}$ ) is required for numerical solution. In addition, collision phenomena are strongly non-linear in such a way that the time-step must be significantly increased to obtain accurate results. In order to opt for a convenient time-step, an analysis on the convergence of simulated results with regard to the choice of different values of time-step is presented. The analysis is done separately for the cases with and without string/string collisions and ignoring the coupling with the body for simplicity. For each case, two-string model simulations are performed using the same initial conditions with four different sampling frequencies, and comparisons are shown in Figure ??. As shown in Figure ??, simulated results without collisions completely converges from $f_{s}=220.5 \mathrm{kHz}$, while results with collisions completely converge from $f_{s}=441.0 \mathrm{kHz}$, as shown Figure ??. Based on these tests and in order to balance calculation time and accuracy, the chosen sampling frequency values for further simulations in this paper will be $f_{s}=$ $220.5 \mathrm{kHz}$ for cases without collisions, and $f_{s}=441.0 \mathrm{kHz}$ for cases with collisions.

\subsubsection{Results: organization of collisions in space and time}

The organization of collisions in space and time is investigated by considering a pair of strings with simply supported boundary conditions, i.e. without any coupling with the bridge. Figure ?? shows a sequence of snapshots of two colliding strings in the $x y$ plane. After being released at $t=8.0 \mathrm{~ms}$, the excited string behaves as a typical plucked string. A first contact between the plucked string and the string at rest is occurring at $t=12.1 \mathrm{~ms}$. The two strings remain in contact during a short period of time (from $t=12.1 \mathrm{~ms}$ to $t=12.4 \mathrm{~ms}$ ). During this contact phase, the interaction point is moving in the $x$ direction. The two strings finally separate after $t=12.4 \mathrm{~ms}$.

The phenomenon of moving contact point is also shown in Figure ??, which is a space-time diagram representing the occurrence of collisions: each black point in this diagram corresponds to a contact point between the two strings. The space-time diagram reveals that the moving contact point phenomenon is repeated several times, with ever shorter durations involving ever smaller portions of the string. It also reveals that collisions occur only in the immediate transient phase just after the pluck, between $12.5 \mathrm{~ms}$ and $71.6 \mathrm{~ms}$ for the studied case. Two "gaps" are also observed in the third contact phase (see Fig. ??). These gaps can be explained by the fact that the collided string, which is initially at rest, starts vibrating after the first collision. The following contact phases (the third one being the first of them) may therefore be disturbed by short temporary contact losses.

\subsubsection{Collisions effects on the sound}

In order to identify the effects of string/string collisions on sound, a simulation is performed on the same pair of strings by adding the coupling with the body. Three different effects can be distinguished.

\section{Buzzing effect and spectral enrichment}

The repeated collisions in the early transient phase induce a clearly perceptible buzzing effect. This permits to conclude that such buzzing effect constitutes an important sound feature of the viola caipira. In the frequency domain, such effect induces a spectral enrichment, clearly visible in Figure ??. 


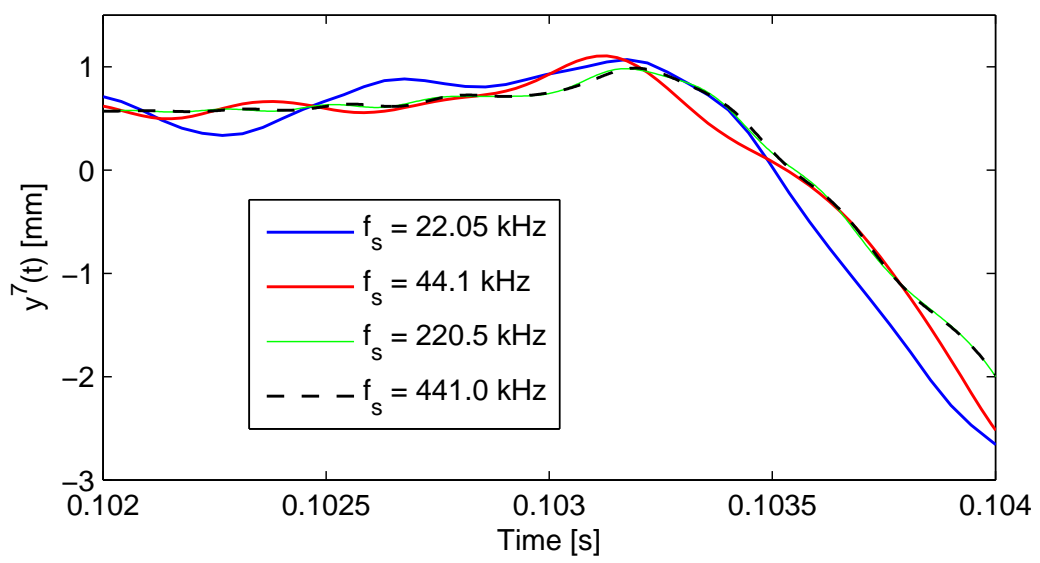

(a) Model without string/string collisions.

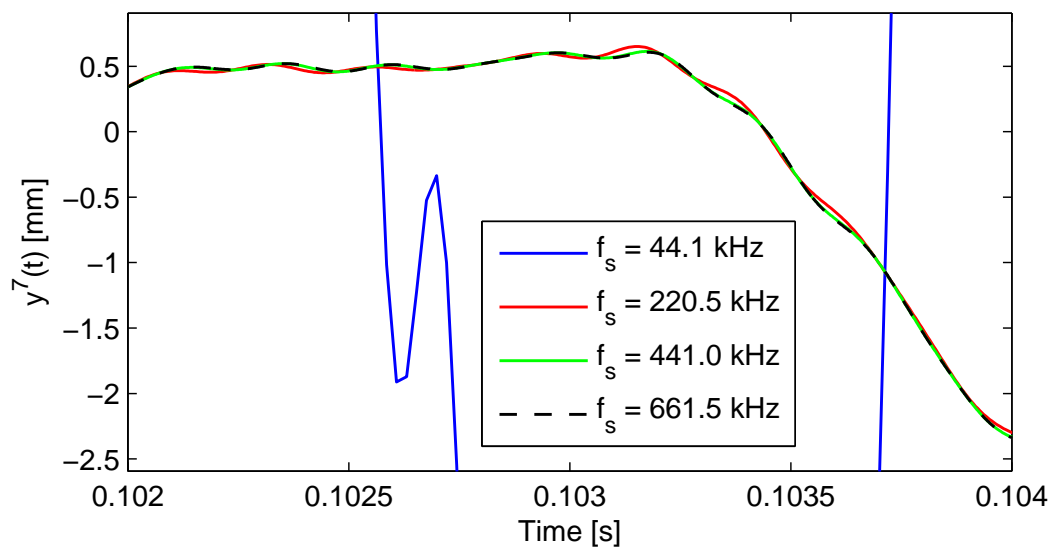

(b) Model with string/string collisions.

Figure 4.7: Tests of convergence: influence of the sampling frequency $f_{s}$. Two-string model with strings 7 and 8. Only string 7 (D3) is plucked, parallel to the soundboard plane. Zoomed numerical displacement of string 7 in the $y$-polarization, at the plucking point, for four sampling frequencies, (a) with collision (b) and without collisions.

\section{Redistribution mechanism of the spectral components}

Figure ?? shows the comparison between two spectrograms of the bridge velocity at the same point, resulting from identical pluck conditions but with or without considering string/string collisions. In both spectrograms, string and body spectral components are clearly distinguishable: contributions due to the string modes coupled with the body are quasi-harmonic with longer duration and contributions due to the body modes are inharmonic with shorter duration. The string being excited approximately at $1 / 7^{\text {th }}$ of its total length, partials multiples of 7 are weak without considering collisions (see Fig. ??). This is not the case anymore when collisions are considered (see Fig. ??). As a conclusion, the comb filtering effect related to the plucking position is canceled due to the collisions. The collisions provide an energy redistribution mechanism which concerns all spectral components since the collision point is moving. 


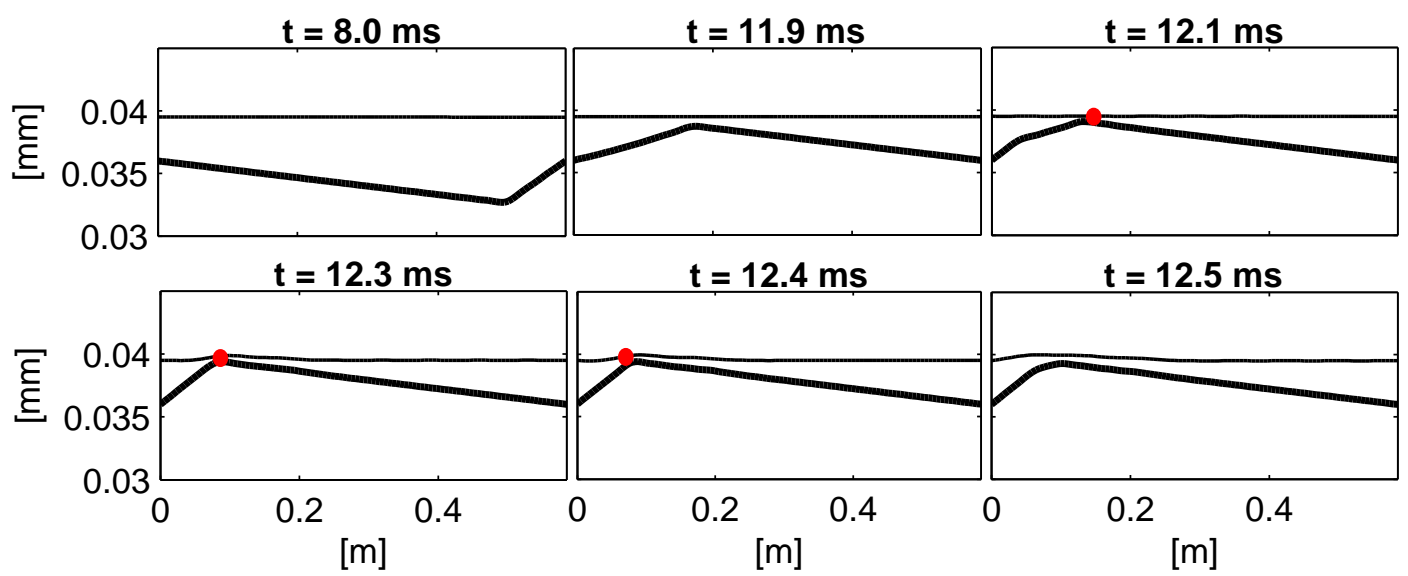

Figure 4.8: Two-string model with colliding strings 7 and 8. Only string 7 (D3) is plucked, parallel to the soundboard plane. Snapshots of the time evolution of the strings in the $x y$ plane.

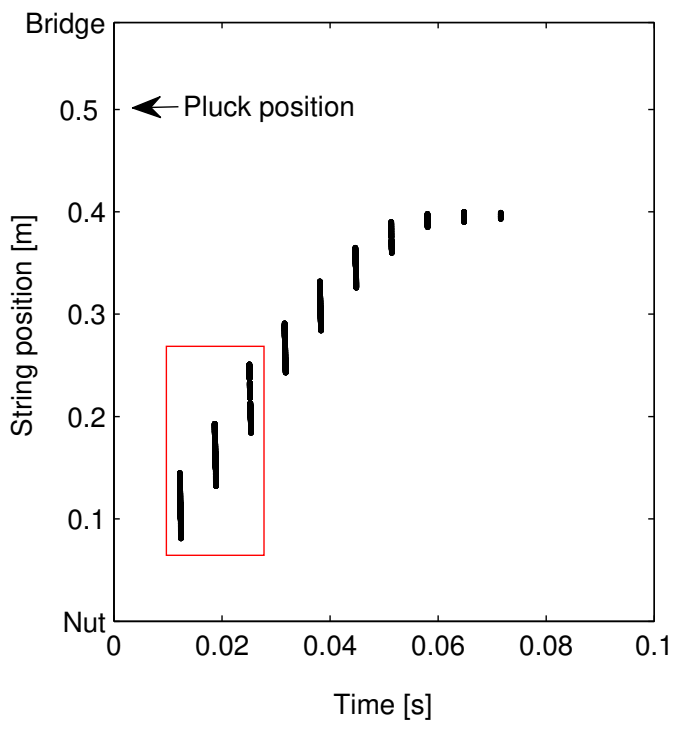

(a)

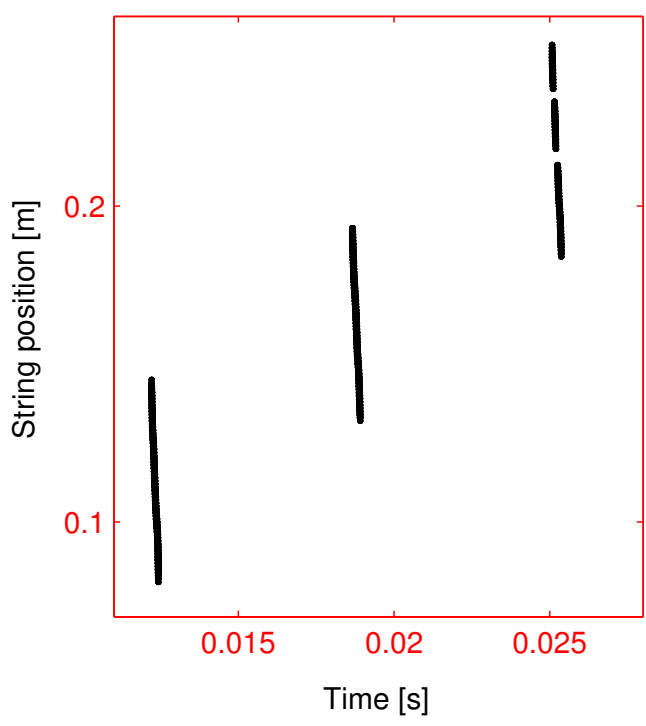

(b)

Figure 4.9: (a) Space-time diagram representing the occurrence of collisions between strings 7 and 8 . Only string 7 (D3) is plucked, parallel to the soundboard plane. (b) Zoom of the rectangle depicted in (a).

\section{Polarization conversion mechanism}

Collisions also induce polarization changes. To highlight this effect, simulations are performed on the same pair of strings without considering the coupling with the body. Figure ?? shows the displacements in the $y$ and $z$ directions, resulting from a pluck either applied in the in-plane direction (Figure ??) or with a small plucking angle $\theta=5^{\circ}$ relative to the $y$ direction (Figure ??). In the first case, the vibration remains in its initial polarization. In the second case, the collisions produce a clear polarization 


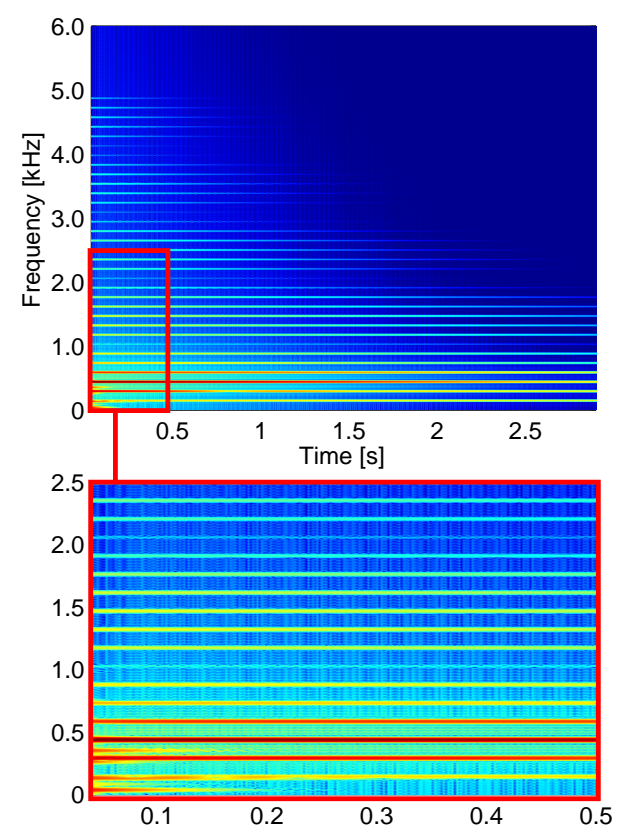

(a) Without collisions.

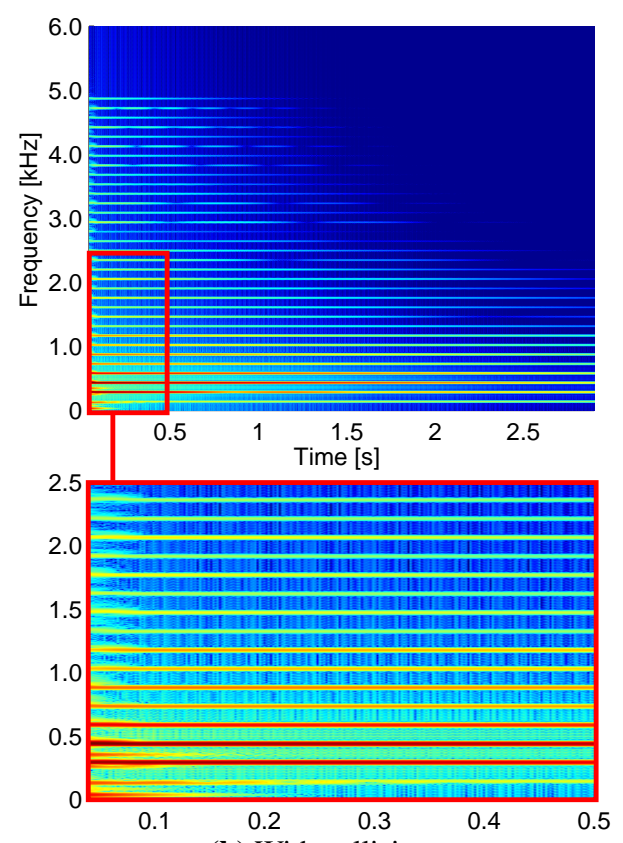

(b) With collisions.

Figure 4.10: Two-string model with strings 7 and 8. Only string 7 (D3) is plucked, parallel to the soundboard plane. Spectrograms of the velocity at the string/body coupling point 7, drawn in $\mathrm{dB}$ using $90 \mathrm{~dB}$ dynamic, without (a) and with (b) string/string collisions.

conversion mechanism. Note that from the displacement time-histories in Figures ?? and ??, and from orbital trajectories in Figure ??, represented on a short time window around two collision instants, it is clear that the plucking angle plays an important role in the polarization change. Note that in the tested configuration, the diameters of the two colliding strings are different (see Table ??).

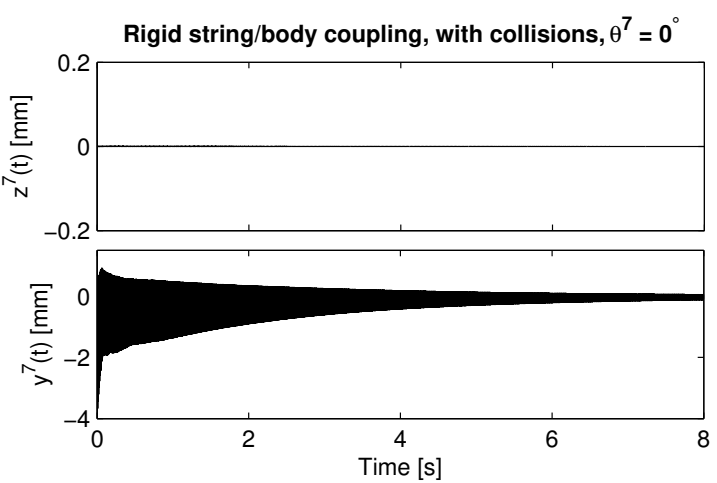

(a)

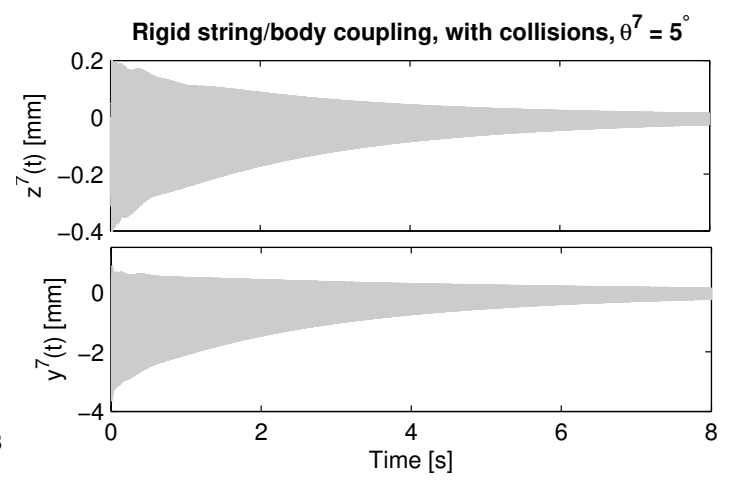

(b)

Figure 4.11: Two-string model considering strings 7 and 8 with completely rigid string/body coupling. Displacement time-history of string 7 (D3) when it is plucked (a) parallel to the soundboard and (b) at $5^{\circ}$ from the negative $y$-axis, in the anti-clockwise. 


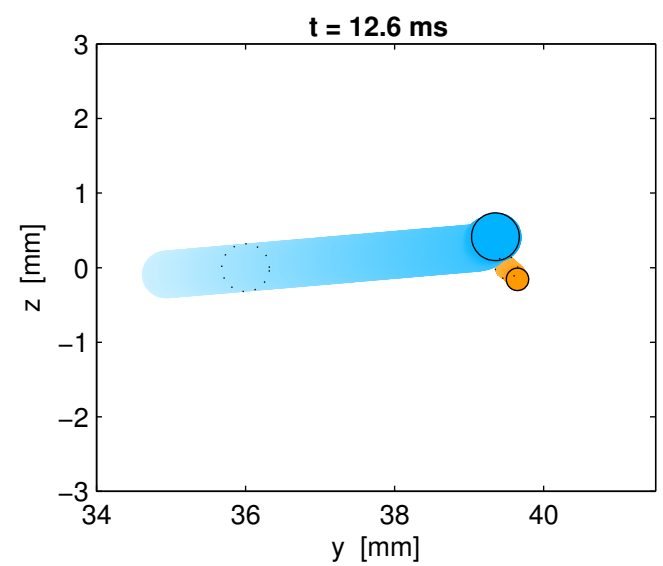

(a)

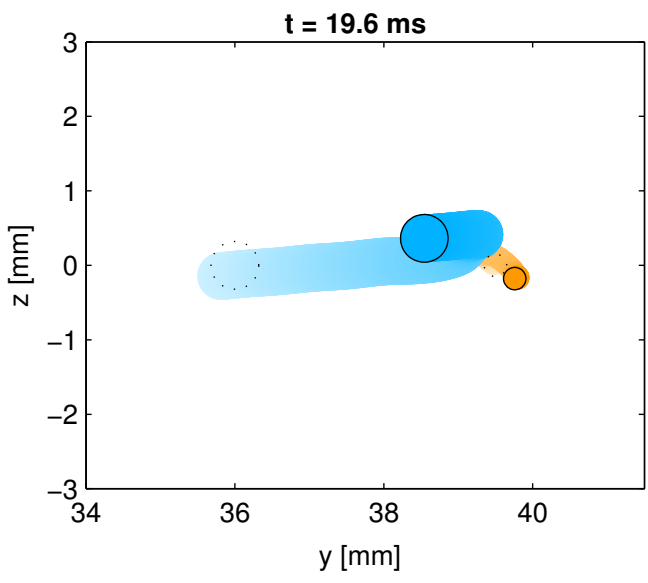

(b)

Figure 4.12: Orbital trajectories in the $y z$ plane at the time indicated of strings 7 (D3) and 8 (D4), represented respectively in blue and orange, at $14 \mathrm{~cm}$ from the nut.Two-string model considering strings 7 (D3) and 8 (D4) is used and string 7 is plucked at $5^{\circ}$ from the negative $y$-axis, in the anti-clockwise.

\subsubsection{Fully coupled system simulations}

The combined effects of the bridge coupling and the collisions are studied in this section. Simulations on a 10-string fully coupled model are performed for three configurations : by considering rigid extremities (Figure ??), by considering the string/bridge coupling with the bridge (Figure ??), by considering both bridge coupling and collisions (Figure ??). In the three cases, only string 7 is plucked parallel to the soundboard and the strings responses are computed in the $y$ and $z$ directions, represented by red and blue curves, respectively.

In Figure ??, the excitation phase is indicated by the shaded area. It is seen that at the end of this phase the plucking force induces a small oscillation in string motion just before the string is released. It is also seen that the only motion is the one of the excited string since no bridge coupling and no collisions are considered.

Comparing the time-history shown in Figures ?? and ?? reveals no significant discrepancy between the two motions in the excitation phase (indicated by the shaded area): the effect of the bridge coupling on the excited string motion is not observed in this excitation phase. In Figure ??, motions of string 8 (D4), which is initially at rest, are observed in the $y$ and $z$ directions. They correspond to sympathetic motions, induced by the bridge coupling. A clear polarization change is observed since these sympathetic vibrations concern both directions. These sympathetic motions of string 8 are slightly increasing over the first $100 \mathrm{~ms}$, which is the signature of the bridge coupling effect.

Comparing the time-history shown in Figures ?? and ?? reveals that the effect of the collisions arises in a very abrupt way. The very first periods of oscillations following the excitation phase are strongly affected and polarization changes occur just after the first collision. It shows the high efficiency of this polarization conversion mechanism. In contrast with this abrupt mechanism, the conversion mechanism coming from the bridge coupling effect arises in a relatively long time scale.

Figure ?? shows the effect of the bridge motion and collisions over a long-term duration (i.e. aftersound). Sympathetic effects, induced in a combined way by the bridge coupling and string/string 


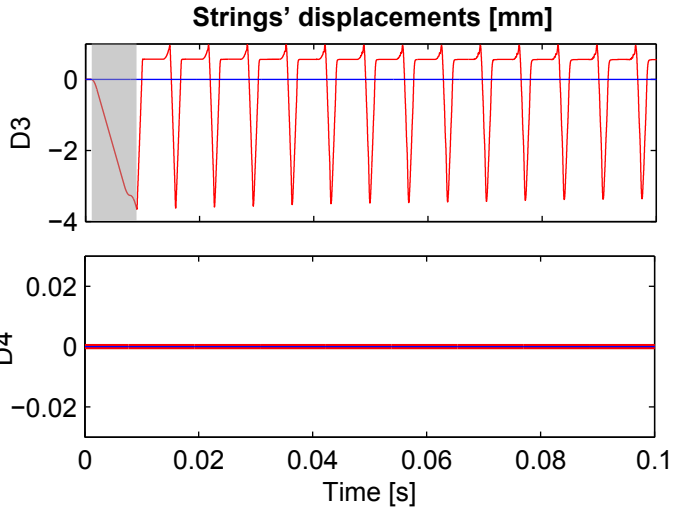

(a) Rigid string/body coupling without collisions

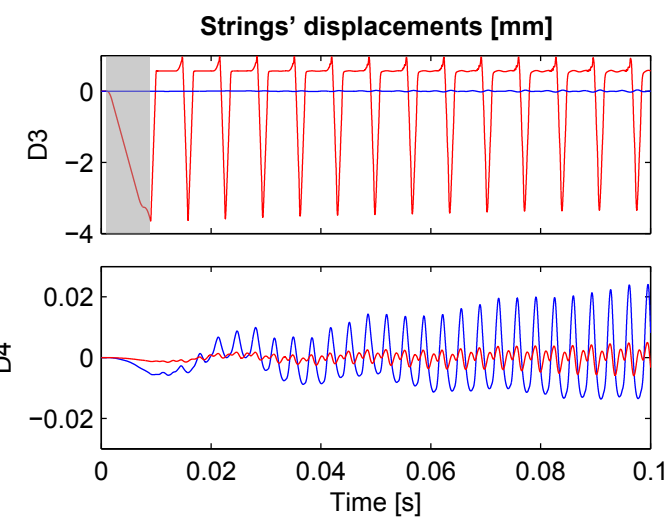

(b) Flexible string/body coupling without collisions

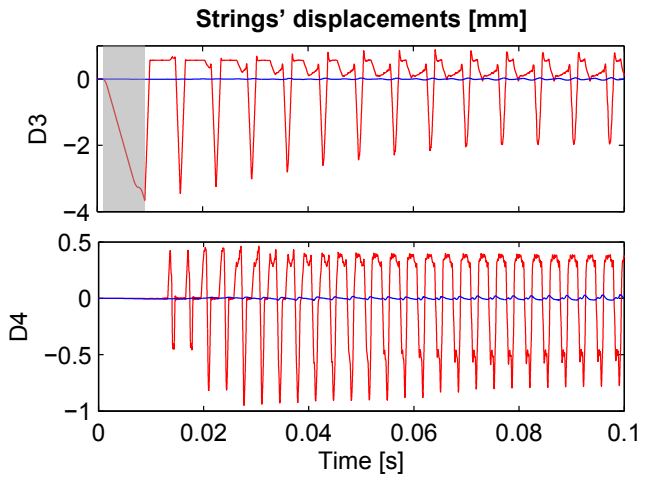

(c) Flexible string/body coupling with collisions

Figure 4.13: 10-string fully coupled model. Zoomed displacement time-histories of strings 7 (D3) and 8 (D4), at $8.5 \mathrm{~cm}$ from the bridge, in the $y$ and $z$ directions, represented by red and blue curves, respectively, when the string 7 is plucked parallel to the soundboard plane. (a) Rigid string/body coupling without collisions; (b) flexible string/body coupling without collisions; (c) flexible string/body coupling with collisions. 

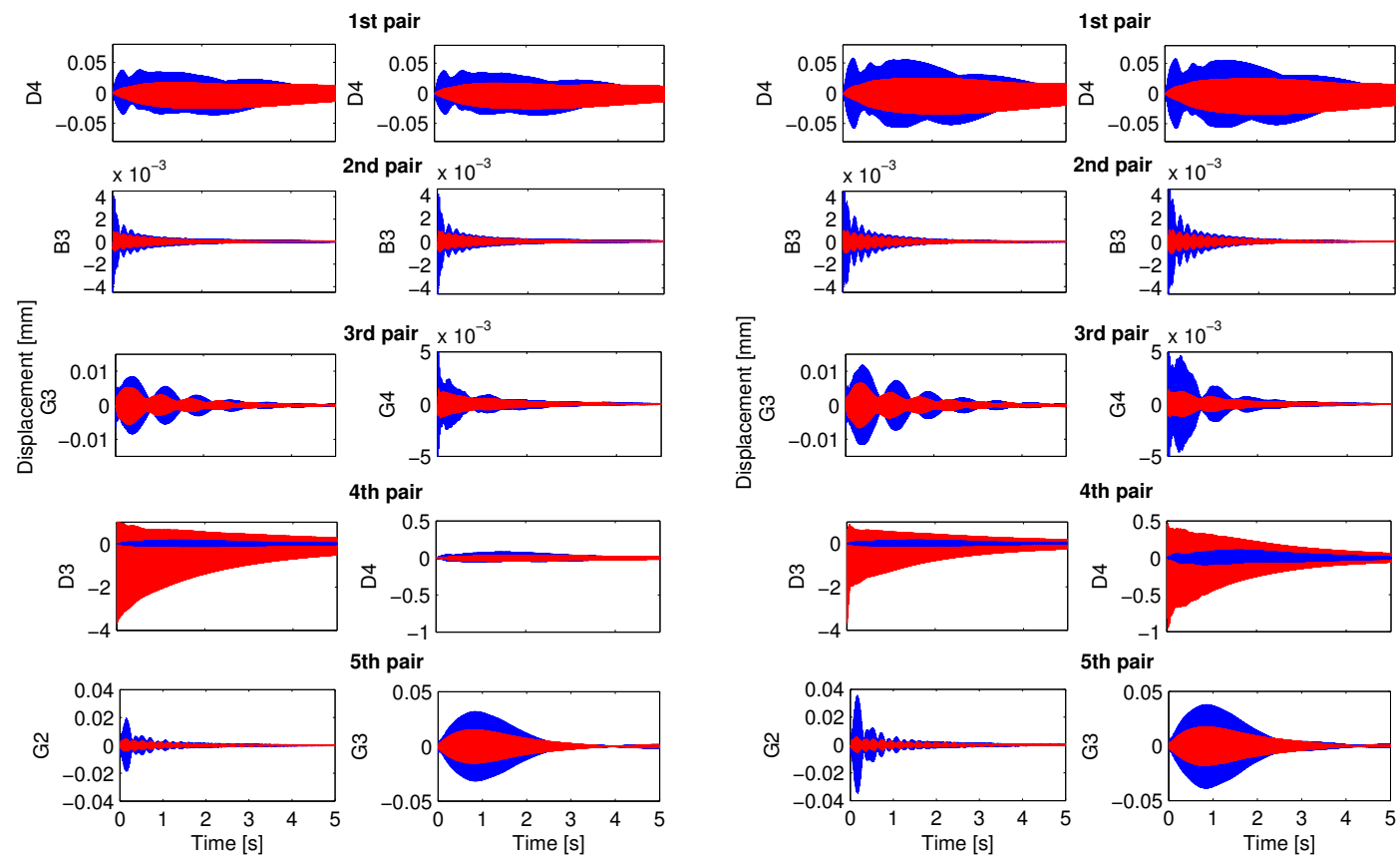

5th pair

(a) Without collisions

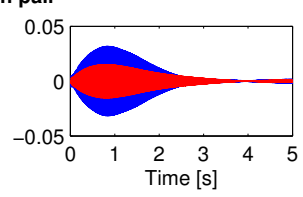

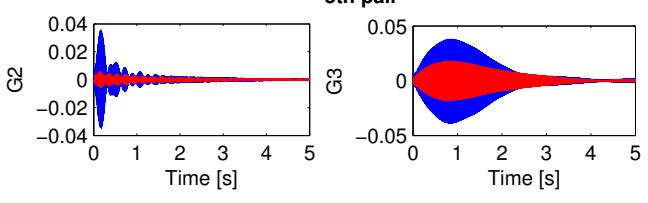

(b) With collisions

Figure 4.14: Displacement time-histories for the 10 strings, at $8.5 \mathrm{~cm}$ from the bridge, in the $y$ and $z$ directions, represented by red and blue curves, respectively, when the string 7 is plucked parallel to the soundboard plane. (a) Model with collisions; (b) model without collisions.

collisions are observed. They are responsible for beating phenomena observed on some strings (see for example G3-G4, B3-B3) which constitute another remarkable feature of the viola caipira. It is shown that even if the collisions are arising only in the early transient phase, its effect concerns the aftersound of all strings and not only the strings pair where the collisions occurred.

\subsection{Conclusion}

In an attempt to identify the specific features of the viola caipira, an experimental analysis of the instrument response to a pluck has been performed using a high speed camera. The analysis reveals that the instrument is characterized by a double pluck excitation since the strings of a given pair are plucked successively and rapidly. Collisions between strings located in the same pair are also revealed. To our knowledge, such collision phenomenon has not been yet reported in the literature.

To investigate more precisely how the collisions affect the string motions, a physical model based on a modal approach is developed for sound synthesis purposes. The model includes 10 strings with non-planar motions coupled with the body and considers collisions between strings. Strings are described analytically whereas modal characteristics of the body are obtained experimentally from mobility measurements. Strings and body are coupled by displacement compatibility at the connection points and a finite difference scheme is used to compute the coupling forces at each time-step, which permits a set of sound simulations.

Besides, the collision effects on the instrument sound are also identified as follows: (i) spectral 
enrichment and buzzing effect in the early transient phase; (ii) energy redistribution between string components; (iii) string polarization changes. The model is able to reproduce the important vibroacoustic features of the viola caipira, among which the sympathetic string resonances and the string/string collisions. Such phenomena constitute the main acoustic signatures of the instrument.

\section{Acknowledgements}

The authors are thankful to Rozini Musical Instruments for providing a viola caipira for the experiments, and to the Brazilian Coordination for the Improvement of Higher Education Personnel Foundation (Grant 99999.010073/2014-00), National Council for Scientific and Technological Development in Brazil (Grant 141214/2013-9) and French National Center for Scientific Research for the financial support.

\subsection{Appendix A: Computation of mass and stiffness matrices of strings}

The mass and stiffness matrices of strings are determined by using energy considerations. For a given string s, the kinematic energy $E_{k i n}^{(s)(w)}$ associated to the motion in the direction $w$ ( $w$ stands for $y$ and $z$ ) is given in terms of the modal amplitudes as follows (see for example [?], Chapter 5):

$$
E_{\text {kin. }}^{(s)(w)}=-\omega^{2} \frac{1}{2} \sum_{j=0}^{N_{(s)}} \sum_{l=0}^{N_{(s)}} m_{j l}^{(s)} a_{j}^{(s)(w)} a_{l}^{(s)(w)},
$$

where

$$
m_{j l}^{(s)}=\mu^{(s)} \int_{0}^{L^{(s)}} \phi_{j}^{(s)(w)} \phi_{l}^{(s)(w)} d x
$$

is the entry in $j^{\text {th }}$ row and $l^{\text {th }}$ column of the submatrix $\mathbf{M}^{(s)(w)}$. Note that $\mathbf{M}^{(s)(w)}$ has non-null elements along its first row and column because the interface and simply supported modes, whose shapes are respectively $\phi_{0}^{(s)}(x)=\left(\frac{x}{L^{(s)}}\right)$ and $\phi_{j}^{(s)}(x)=\sin \left(\frac{j \pi x}{L^{(s)}}\right)$ are not orthogonal with respect to the mass. Accordingly, for a given string $\mathrm{s}$ and polarization $w$, the potential energy is given by

$$
E_{\text {pot. }}^{(s)(w)}=\frac{1}{2} \sum_{j=0}^{N_{(s)}} \sum_{l=0}^{N_{(s)}} k_{j l}^{(s)} a_{j}^{(s)(w)} a_{l}^{(s)(w)}
$$

where

$$
k_{j l}^{(s)}=T^{(s)} \int_{0}^{L^{(s)}} \frac{\partial \phi_{j}^{(s)(w)}}{\partial x} \frac{\partial \phi_{j}^{(s)(w)}}{\partial x} d x+B^{(s)} \int_{0}^{L^{(s)}} \frac{\partial^{2} \phi_{j}^{(s)(w)}}{\partial x^{2}} \frac{\partial^{2} \phi_{j}^{(s)(w)}}{\partial x^{2}} d x
$$

is the entry in $j^{\text {th }}$ row and $l^{\text {th }}$ column of the submatrix $\mathbf{K}^{(s)(w)}$. 


\subsection{Appendix B: String damping model}

The string modal Q-factors in Equation ?? are computed following the model presented in [?] and recently adopted in $[?, ?]$. For mode $j$ of a given string $s$, the Q-factor expression is modelled as:

$$
\left(Q_{j}^{(s)}\right)^{-1}=\left(Q_{j, \text { air }}^{(s)}\right)^{-1}+\left(Q_{j, \text { ve } / t e}^{(s)}\right)^{-1}+\left(Q_{\text {disl. }}^{(s)}\right)^{-1}
$$

where the first and second terms in the right-hand side are related to the air viscosity and visco and thermoelastic losses. The third term, $\left(Q_{\text {disl }}^{(s)}\right)^{-1}=\delta_{\text {disl. }}$, is due to the dislocation phenomenon, which is assumed constant over the audio frequency range [?]. The contribution due to the air viscosity writes:

$$
Q_{j, \text { air }}^{(s)}=\frac{R^{(s)}}{\mu^{(s)}} \frac{1}{\omega_{j}^{(s)}},
$$

where $R^{(s)}=2 \pi \eta_{\text {air }}+2 \pi d^{(s)}\left(\frac{\eta_{\text {air }} \rho_{\text {air }} \omega_{j}^{(s)}}{2}\right)^{1 / 2}$ with $\rho_{\text {air }}$ density of the air and $\eta_{\text {air }}$ the dynamic viscosity coefficient. Contributions due to visco and termoelastic losses are given by:

$$
Q_{j, v e / t e}^{(s)}=\frac{E^{(s)} I^{(s)}}{\left(T^{(s)}\right)^{2} c^{(s)}}\left(\delta_{v e / t e}\right)\left(\omega_{j}^{(s)}\right)^{2},
$$

where $\delta_{v e / t e}=\delta_{v e}+\delta_{t e}$, with $\delta_{v e}$ and $\delta_{t e}$ the visco and termoelastic loss angles, respectively, $E^{(s)}$ is the Young's modulus and $I^{(s)}=\frac{\pi\left(d^{(s)} / 2\right)^{4}}{4}$ is moment of inertia of the string.

\subsection{Appendix C: Coefficients for computation of string/body coupling forces}

Table ?? gives the expressions to calculate the coefficients of Equation ??, used to obtain the string/body coupling forces at each time-step. Note that for the cases where string/string collisions are considered, the coefficients in red are included in the computation of the matrices $\mathbf{A}$ and $\boldsymbol{\alpha}$.

Finally, the matrices $\boldsymbol{\Phi}_{\mathbf{e}}^{\mathbf{s}}, \boldsymbol{\Phi}_{\mathbf{c}}^{\mathbf{s}}$ and $\boldsymbol{\Phi}_{\text {imp }}^{\mathbf{s}}$ are given as follows:

$$
\begin{aligned}
\boldsymbol{\Phi}_{\mathbf{e}}^{\mathbf{s}} & =\left[\begin{array}{ccccc}
\boldsymbol{\phi}^{1 y}\left(x_{e}^{1}\right) & & & & \\
& \boldsymbol{\phi}^{1 z}\left(x_{e}^{1}\right) & & (\mathbf{0}) & \\
& & \ddots & & \\
& (\mathbf{0}) & & \boldsymbol{\phi}^{10 y}\left(x_{e}^{10}\right) & \boldsymbol{\phi}^{10 z}\left(x_{e}^{10}\right)
\end{array}\right], \\
\boldsymbol{\Phi}_{\mathbf{c}}^{\mathbf{s}} & =\left[\begin{array}{ccccc}
\boldsymbol{\phi}^{1 y}\left(x_{c}^{1}\right) & & & & \\
& \boldsymbol{\phi}^{1 z}\left(x_{c}^{1}\right) & & (\mathbf{0}) & \\
& & \ddots & & \\
& (\mathbf{0}) & & \boldsymbol{\phi}^{10 y}\left(x_{c}^{10}\right) & \boldsymbol{\phi}^{10 z}\left(x_{c}^{10}\right)
\end{array}\right]
\end{aligned}
$$


TABLE 4.3: Coefficients of Equation ?? for computation of the string/body coupling forces.

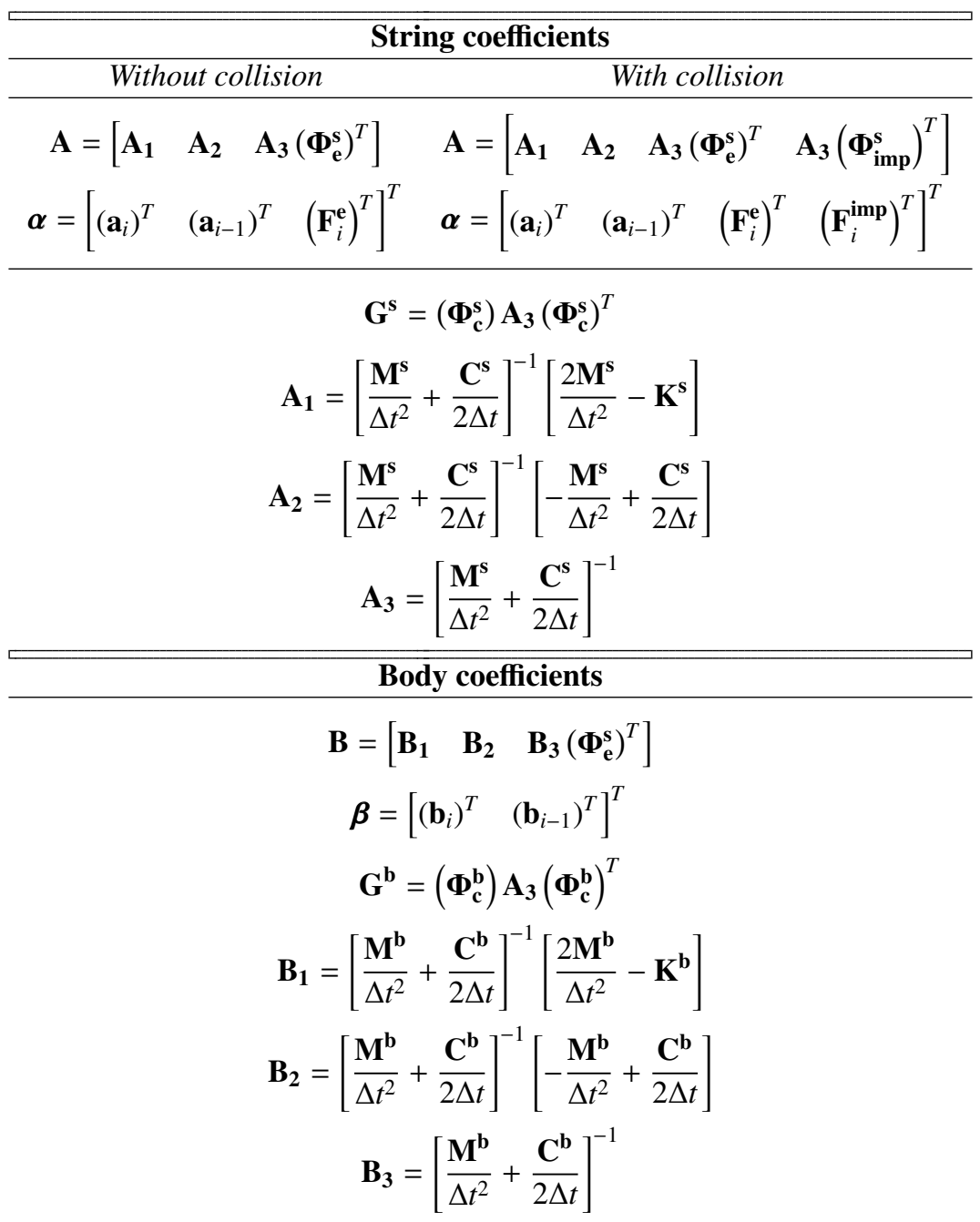

and

$$
\Phi_{\text {imp }}^{\mathrm{s}}=\left[\begin{array}{ccccc}
\Phi_{\mathrm{imp}}^{1 \mathrm{y}} & & & & \\
& \Phi_{\mathrm{imp}}^{1 \mathrm{z}} & & (0) & \\
& & \ddots & & \\
& (0) & & \Phi_{\mathrm{imp}}^{10 y} & \\
& & & & \Phi_{\text {imp }}^{10 \mathrm{z}}
\end{array}\right],
$$

where

$$
\boldsymbol{\Phi}_{\mathbf{i m p}}^{(s)(w)}=\left[\begin{array}{lll}
\boldsymbol{\phi}^{(s)(w)}\left(x_{1}\right) & & \\
& \ddots & \\
& & \boldsymbol{\phi}^{(s)(w)}\left(x_{M(s)}\right)
\end{array}\right],
$$

with $\boldsymbol{\phi}^{(s)(w)}\left(x_{n}\right)$ the mode shape of the $s^{\text {th }}$ string in the polarization $w$ at the $n^{\text {th }}$ point $(n=1, \ldots, M(s))$. 


\section{Complements}

\subsection{Complement I: Double pluck synthesis}

In order to further explore the sound synthesis model developed in this chapter, complementary simulations are presented below. As observed experimentally in Section ??, the viola caipira is characterized by a double pluck since the strings of a given pair are plucked rapidly and successively in a short time interval denoted by $\Delta t_{\text {pluck }}=t_{r}^{2 n d}-t_{r}^{1 s t}$, where $t_{r}^{2 n d}$ and $t_{r}^{1 s t}$ are the instants at which the second and first plucked strings of a given pair are released, respectively. The main goal of this complement is to illustrate the influence of such double pluck conditions on the sound produced. The $4^{\text {th }}$ string pair $(s=7,8)$ is considered for the simulations presented below. Strings 8 (D4) and 7 (D3) are plucked successively and the following excitation parameters are varied:

- Pluck positions $x_{e}^{(s)}$;

- Maximum excitation force amplitudes $F_{0}^{(s)}$ and pluck interval $\Delta t_{p l u c k}=t_{r}^{8}-t_{r}^{7}$.

\subsubsection{Variation of the pluck position}

Strings 8 (D4) and 7 (D3) are plucked at the same position with three variations of the pluck positions are selected: $14.5 \mathrm{~cm}, 8.5 \mathrm{~cm}$ and $2.5 \mathrm{~cm}$ from the bridge. The other pluck parameters are constant as shown in Table ??.

TABLE 4.4: Double pluck parameters. Strings 8 and 7 are plucked successively.

\begin{tabular}{ccccccc}
\hline$F_{0}^{8}[\mathrm{~N}]$ & $F_{0}^{7}[\mathrm{~N}]$ & $t_{i}^{8}[\mathrm{~ms}]$ & $t_{r}^{8}[\mathrm{~ms}]$ & $t_{i}^{7}[\mathrm{~ms}]$ & $t_{r}^{7}[\mathrm{~ms}]$ & $\Delta t_{\text {pluck }}[\mathrm{ms}]$ \\
\hline 3 & 3 & 1 & 6 & 14 & 20 & 14 \\
\hline
\end{tabular}

Figures ??, ?? and ?? show the space-time diagrams representing the occurrence of collisions along the strings' lengths where the vertical red dashed lines depict the release instant of the second plucked string (string 7). Figures ??, ?? and ?? show the respective spectrograms of the bridge velocity at the string/body coupling 7 calculated in the first $900 \mathrm{~ms}$.

The influence of the pluck position on the sound produced can be clearly observed from the results comparison. Collisions do not occur when the strings are plucked $2.5 \mathrm{~cm}$ from the bridge since their vibration amplitudes are relatively smaller. On the other hand, when the strings are excited in positions farther from the bridge, their vibration amplitudes are relatively higher, leading to successive collisions. In addition, the spatial distribution of collisions increases significantly when the string is excited at 14.5 $\mathrm{cm}$ from the bridge. As consequence, different patterns of energy redistribution due to the string/string collisions are seen in Figures ?? and ??.

\subsubsection{Variation of the maximum excitation force amplitudes $F_{0}^{(s)}$ and pluck interval $\Delta t_{\text {pluck }}$}

Strings 8 (D4) and 7 (D3) are plucked using three variations of gestures increasingly abrupt as follows: $F_{0}^{(s)}=2.8 \mathrm{~N}$ and $\Delta t_{\text {pluck }}=16 \mathrm{~ms}, F_{0}^{(s)}=3.2 \mathrm{~N}$ and $\Delta t_{\text {pluck }}=12 \mathrm{~ms}, F_{0}^{(s)}=3.4 \mathrm{~N}$ and $\Delta t_{\text {pluck }}=10 \mathrm{~ms}$. The pluck parameters shown in Table ?? are kept constant.

In order to illustrate the influence of the gesture on the sound produced, an analysis similar to that presented in Subsection ?? is performed: Figures ??, ?? and ?? show the space-time diagrams representing the occurrence of collisions along the strings' lengths where the vertical red dashed lines 
TABLE 4.5: Double pluck parameters. Strings 8 and 7 are plucked successively.

\begin{tabular}{ccc}
\hline$x_{e}^{(s)}[\mathrm{m}]$ & $t_{i}^{8}[\mathrm{~ms}]$ & $t_{i}^{7}[\mathrm{~ms}]$ \\
\hline 0.5 & 1 & 14
\end{tabular}

depict the release instant of the second plucked string (string 7). Figures ??, ?? and ?? show the respective spectrograms of the bridge velocity at the string/body coupling 7 calculated in the first $900 \mathrm{~ms}$.

From the results comparison, it is clearly visible that the gesture variation leads to significant changes of the collisions time-space distribution and, consequently, different sounds are produced. The repeated collisions in the early transient phase induce a clearly perceptible buzzing effect, which can be seen as the enlargement of the spectral rays during the collision events. Note that such enlargement is more accentuated in Figure ??, when the strings are plucked using $F_{0}^{(s)}=3.2 \mathrm{~N}$ and $\Delta t_{\text {pluck }}=12 \mathrm{~ms}$. 


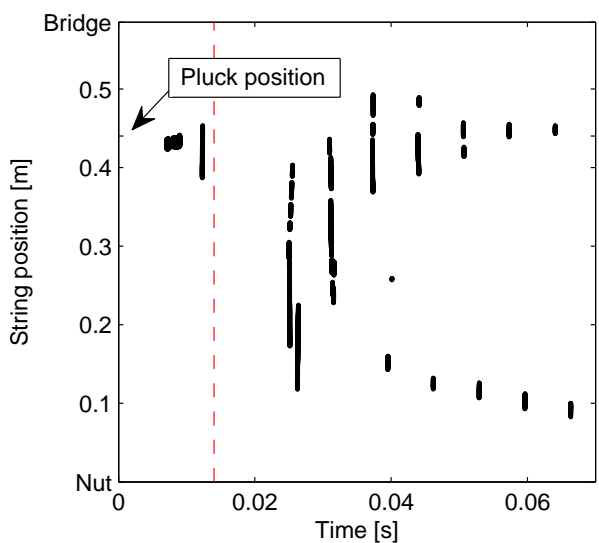

(a) Pluck at $14.5 \mathrm{~cm}$ from the bridge.

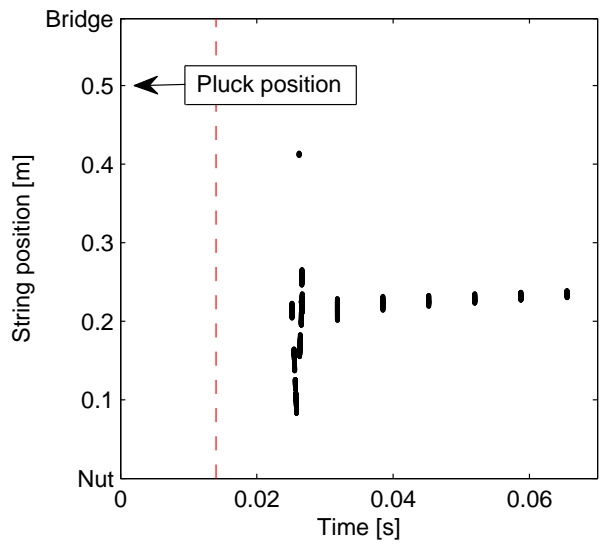

(c) Pluck at $8.5 \mathrm{~cm}$ from the bridge.

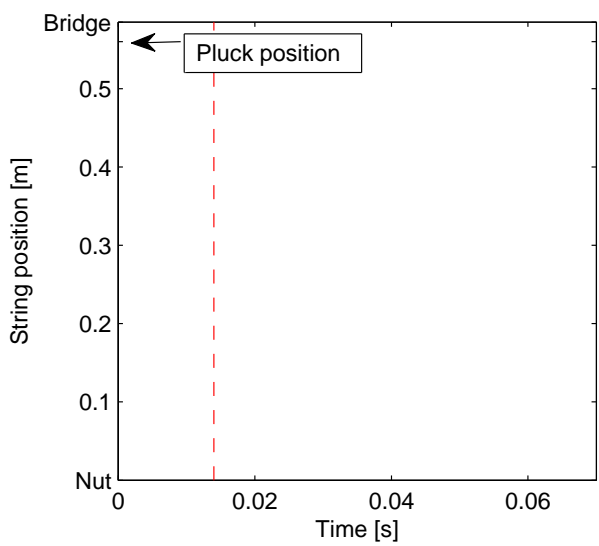

(e) Pluck at $2.5 \mathrm{~cm} \mathrm{~cm}$ from the bridge.

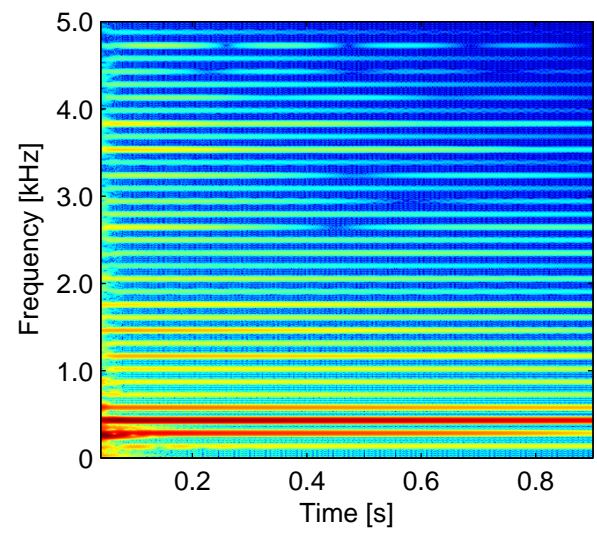

(b) Pluck at $14.5 \mathrm{~cm}$ from the bridge.

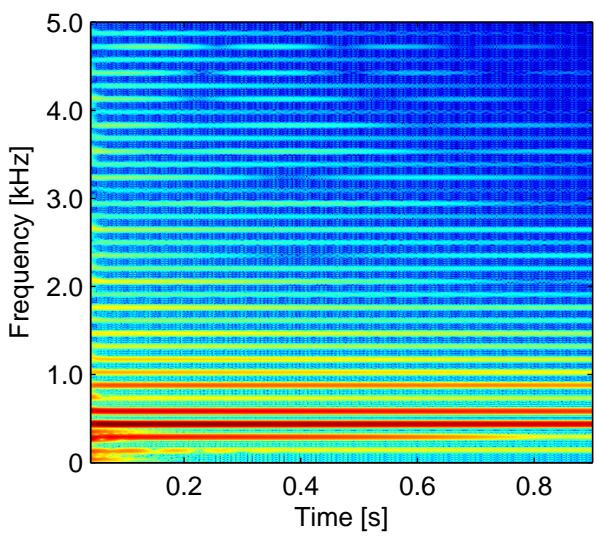

(d) Pluck at $8.5 \mathrm{~cm}$ from the bridge.

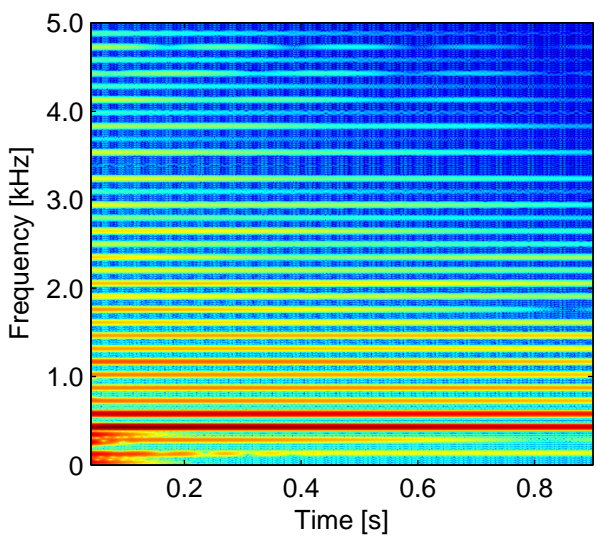

(f) Pluck at $2.5 \mathrm{~cm}$ from the bridge.

Figure 4.15: Double pluck simulations when pluck positions are varied: strings 8 (D4) and 7 (D3) are plucked successively. Space-time diagrams representing the occurrence of collisions when strings are plucked at (a) $14.5 \mathrm{~cm}$ (c) $8.5 \mathrm{~cm}$ and (e) $2.5 \mathrm{~cm}$ from the bridge; (b), (d) and (f) respective spectrograms of the bridge velocity at the coupling point 7 . 


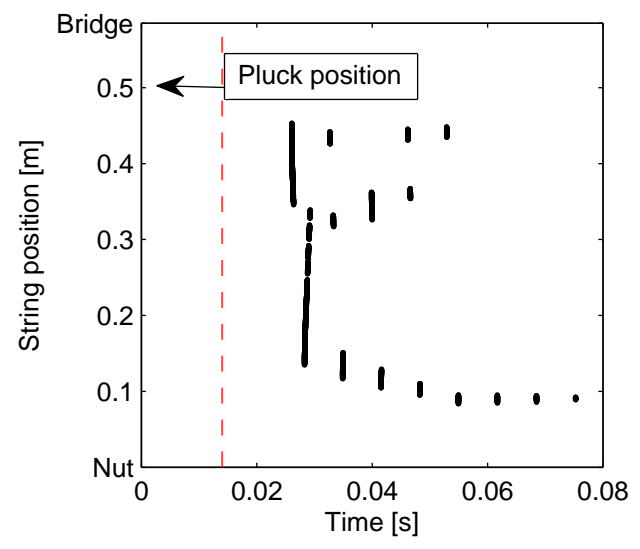

(a) $\Delta t_{\text {pluck }}=16 \mathrm{~ms}, F_{0}^{(s)}=2.8 \mathrm{~N}$.

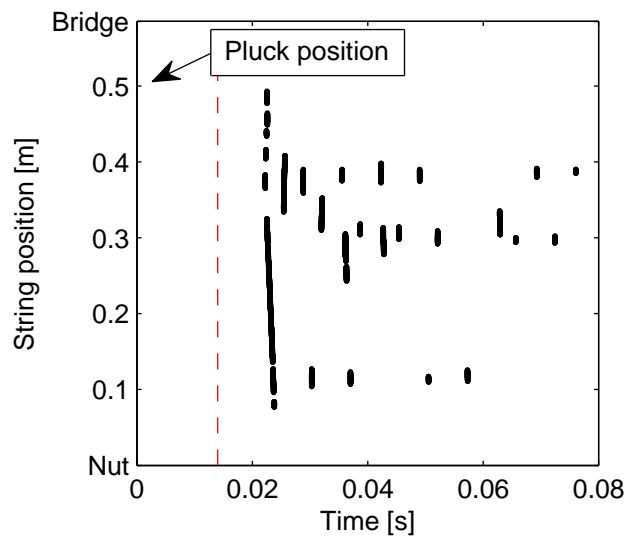

(c) $\Delta t_{\text {pluck }}=12 \mathrm{~ms}, F_{0}^{(s)}=3.2 \mathrm{~N}$.

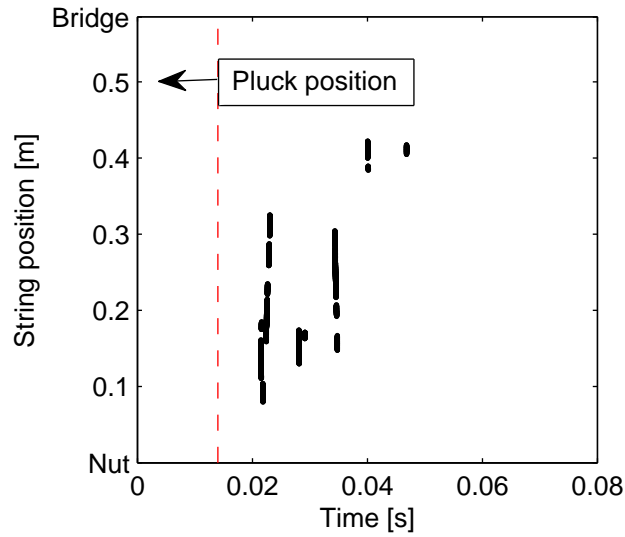

(e) $\Delta t_{\text {pluck }}=10 \mathrm{~ms}, F_{0}^{(s)}=3.4 \mathrm{~N}$.

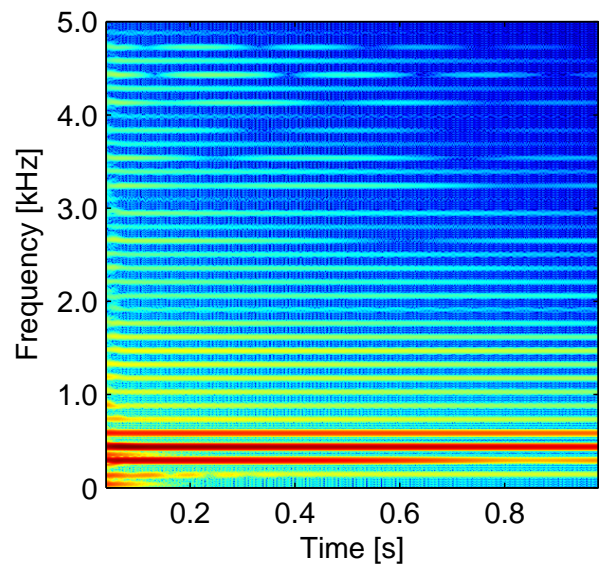

(b) $\Delta t_{\text {pluck }}=16 \mathrm{~ms}, F_{0}^{(s)}=2.8 \mathrm{~N}$.

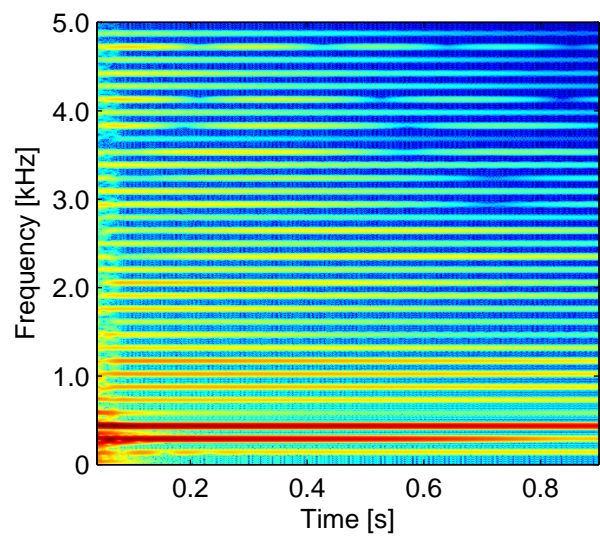

(d) $\Delta t_{\text {pluck }}=12 \mathrm{~ms}, F_{0}^{(s)}=3.2 \mathrm{~N}$.

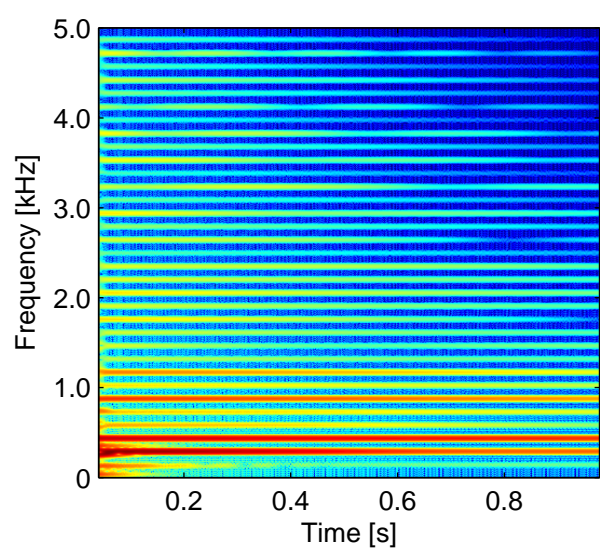

(f) $\Delta t_{\text {pluck }}=10 \mathrm{~ms}, F_{0}^{(s)}=3.4 \mathrm{~N}$.

Figure 4.16: Double pluck simulations when $F_{0}^{(s)}$ and $\Delta t_{\text {pluck }}$ are varied: strings 8 (D4) and 7 (D3) are plucked successively. Space-time diagrams representing the occurrence of collisions when strings are plucked using (a) $F_{0}^{(s)}=2.8 \mathrm{~N}$ and $\Delta t_{\text {pluck }}=16 \mathrm{~ms}$, (c) $F_{0}^{(s)}=3.2 \mathrm{~N}$ and $\Delta t_{\text {pluck }}=12 \mathrm{~ms}$, and (e) $F_{0}^{(s)}=3.4 \mathrm{~N}$ and $\Delta t_{\text {pluck }}=10$ $\mathrm{ms}$; (b), (d) and (f) respective spectrograms of the bridge velocity at the coupling point 7 . 



\section{Chapter 5}

\section{GENERAL CONCLUSIONS AND PERSPECTIVES}

The viola caipira is a guitar played in various genres of traditional and modern Brazilian music, and whose practice extends across all regions of Brazil, being considered one of the Brazilian cultural symbols. Variants of the instrument with different resonance box shapes, tuning types, materials and arrangement of strings are commonly encountered in different regions of the country. In spite of the considerable differences between the varieties of violas caipiras, a very representative configuration of the instrument has been investigate in this thesis. It has five courses of double metallic strings: the first two courses have identical strings tuned in unison while the other three have strings with different diameters tuned in an octave, which makes the instrument strings more susceptible to vibrate sympathetically.

Unlike other string instruments such as classical guitars and violins, the viola caipira is a little explored instrument from the perspective of musical acoustics. To the author's knowledge, this thesis work is the first attempt in the literature to characterize the instrument in vibrational and acoustical terms with the objective of identifying its specificities. Such investigation has been limited to the physical characterization of the instrument involving different methods such as vibration and sound pressure measurements, investigation of string motion using high speed camera, physical modelling for sound synthesis purposes and numerical modelling using the finite element method.

In an attempt to identify the specific features of the viola caipira, an experimental study of the instrument using different methods has been presented as follows:

- An experimental analysis of the instrument response to a pluck has been performed using a high speed camera. The analysis reveals that the instrument is characterized by a double pluck excitation since the strings of a given pair are plucked successively and rapidly. Collisions between strings located in the same pair are also revealed. Such collision phenomenon has not been reported in the literature before.

- An analysis of viola caipira sounds has been performed using time-frequency representations and energy decay curves. It has been identified the existence of string sympathetic resonances and beating phenomena when a single string is plucked and all the other strings are free to vibrate. This phenomenon is perceived as a sound halo, which constitutes an important sound characteristic of the instrument.

- A modal analysis of the viola caipira soundboard carried out by the classical hammer method has revealed some differences and similarities with the classical guitar: the first four modes of the viola caipira soundboard resemble those of the classical guitar while frequencies are moderately higher, which is due to its smaller resonance box. In addition, the viola caipira soundboard, like classical guitar soundboards have been shown to plate-like systems since their mean mobilities are nearly independent on the frequency. Measurements using a scanning laser vibrometer combined with an 
automatic impact hammer have been performed to obtain the ODSs of the front of the viola caipira. At a set of resonance peaks the obtained ODSs give results close to those obtained with the classical modal analysis using impact hammer and accelerometer.

- It has been investigated the capability of the wire-breaking method to accurately obtain the mobilities transfer functions at the bridge of string instruments. Since no force sensor is required, this methodology is a low cost and well-adapted procedure for measurements in the environment of instrument maker workshop. The method was shown to be repeatable and provided results in reasonable agreement with the classical hammer method. A calibration method for mobilities obtained from wire-breaking measurement was proposed and validated. Finally, a modal analysis of the mobility curves measured at the viola caipira bridge using the "Roving Wire-Breaking Technique" allowed the estimation of natural frequencies, damping factors and mode shapes at the string/bridge coupling points using a high resolution modal analysis (ESPRIT method). Such results can be used to feed sound synthesis models.

In order to investigate more precisely how the collisions affect the string motions, a physical model based on a modal approach has been developed for sound synthesis purposes. The model includes 10 strings with non-planar motions coupled with the body and considers collisions between strings. Strings are described analytically whereas modal characteristics of the body are obtained experimentally from mobility measurements. Strings and body are coupled by displacement compatibility at the connection points and a finite difference scheme is used to compute the coupling forces at each time-step, which permits to calculate a set of string responses to a given excitation.

Effects of string/string collisions on the viola caipira sound have been identified as follows:

- The repeated collisions in the early transient phase induce a clearly perceptible buzzing effect. This permits to conclude that such buzzing effect constitutes an important sound feature of the viola caipira. It has been observed that, in the frequency domain, such effect induces a spectral enrichment.

- The comb filtering effect related to the plucking position is canceled due to the collisions. The collisions between strings lead to an energy redistribution mechanism which concerns all spectral components since the collision points move along the string.

- Collisions can also induce polarization changes: the very first periods of oscillations following the excitation phase are strongly affected and polarization changes occur just after the first collision since a small plucking angle relative to the soundboard plane is applied. No polarization changes occur for an excitation parallel to the soundboard, which permits to conclude that the plucking angle plays an important role in such phenomenon. It has been observed that the conversion mechanism coming from the bridge coupling effect arises in a relatively long time scale compared to the abrupt polarization changes that may occur due to the collisions between strings.

Finally, the proposed synthesis model is also able to reproduce string sympathetic resonances, which is another main feature of the viola caipira sound. It has been observed that such sympathetic effects are induced in a combined way by the bridge coupling and string/string collisions. They are responsible for beating phenomena observed on some strings.

One possible application of synthesis models, and in particular hybrid models, is to understand the influence of different physical phenomena on the sound perceived. Identifying the audible and nonaudible elements helps to judge the degree of precision that should be given to a model. We have endeavoured to do this in terms of taking into account the phenomena of sympathetic coupling and collisions, which are clearly important. It would be interesting to take into account the geometrical nonlinearities induced by the large-amplitude vibrations of the strings that take place in the early transient phase. Besides, the inclusion of a sound radiation model to compute the pressure-field resulting from 
the instrument pluck is also suggested. And, finally, an accurate determination of the string damping parameters and impact parameters can allow the experimental validation of the model, which would improve the realism of the synthesized sounds. 



\section{Appendix A}

\section{COMPARISONS BETWEEN SOUND SYNTHESIS METHODS}

This complement compares the results obtained with the sound synthesis method proposed in this thesis, here called the "coupling force computation method" (CFC method) with the results obtained with another modal-based method presented in [?], here called the "coupled modes computation method" (CMC method), which is briefly described in Chapter ??, Subsection ??. Although their differences, these methods start from the same information: input body admittance measured at the bridge and string properties. Body modal parameters are extracted from such admittance measurements and analytical expressions of mode shapes, natural frequencies and damping factors are used to obtain the string modal basis. In order to clarify their differences, the main ideas behind both methods can be summarized as follows:

- $C M C$ method [?]: it consists in computing the string/body coupled modes using a first-order formulation, which allows to obtain string responses by modal superposition;

- CFC method: it does not deal with the computation of string/body coupled modes. Uncoupled strings and body are described separately in term of modal equations and connected by means of a coupling force term, which is external and unknown. The coupling force is finally computed using a finite difference scheme.

Figure ?? shows the comparison of simulations obtained with the two methods fed by the same body and string parameters, as presented in Section ??. Only one string (string 1, D4) with planar motion in the $z$ direction is considered. The string is plucked parallel to the soundboard. and the excitation point is located at $8.5 \mathrm{~cm}$ from the bridge. The same excitation model adopted for simulations in Chapter ?? is used. It can be seen from Figure ?? that both methods lead to close results. 


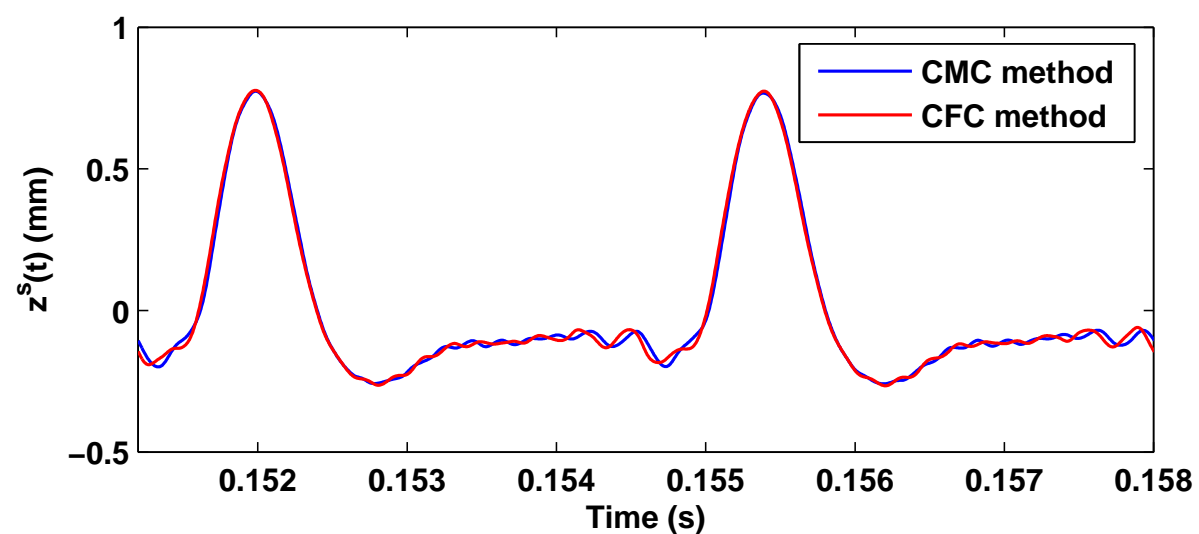

Figure A.1: Comparison of results obtained with the CMC and the CFC methods. One-polarization model of a single string. Zoomed displacement of string 1 (D4) plucked at $8.5 \mathrm{~cm}$ from the bridge, normal to the soundboard plane. 


\section{Appendix B}

\section{INFLUENCE OF THE NUMBER OF STRING AND BODY MODES ON THE STRING RESPONSE}

This complement aims to briefly investigate the influence of the number of string and body modes on the simulated results obtained with the synthesis method proposed in this chapter. String and body modal parameters are obtained using the same procedures as described in Section ??. The same string and plucking conditions of the previous complement are used.

\section{B.1 Number of string modes}

The number of body modes is first set equal to $36(0 \mathrm{~Hz}-1500 \mathrm{~Hz})$ while the maximum frequency for the string harmonics, denoted by $f_{\text {hmax }}$, is set to four different values: $f_{\text {hmax }}=2000 \mathrm{~Hz}, 4000 \mathrm{~Hz}$, $10000 \mathrm{~Hz}$ and $20000 \mathrm{~Hz}$. Figure ?? shows the comparison of the zoomed displacements obtained for the different values of $f_{\text {hmax }}$. Results for $f_{\text {hmax }}=10000 \mathrm{~Hz}$ (black line) and $f_{\text {hmax }}=20000 \mathrm{~Hz}$ (red line) are clearly identical for the selected time interval, while for $f_{\text {hmax }}=4000 \mathrm{~Hz}$ (green line) a small difference is observed. For $f_{\text {hmax }}=2000 \mathrm{~Hz}$ (blue line) the difference is considerable compared to the other curves in this time interval.

Figure ?? compares the spectra associated to the four values of $f_{\text {hmax }}$, taking into account the first $300 \mathrm{~ms}$ of the respective simulated displacements referred above. The spectra for $f_{\text {hmax }}=10000 \mathrm{~Hz}$ and $f_{\text {hmax }}=20000 \mathrm{~Hz}$ (Figures ??a and ??b) present identical profiles. These two spectra in comparison with that of $f_{\text {hmax }}=4000 \mathrm{~Hz}$ (Figure ??c) have a quite small difference of level from $4000 \mathrm{~Hz}$, as highlighted with a dashed circle in Figure ??c. Although a perception analysis is not made here, such differences might be in general inaudible. On the other hand the spectrum for $f_{\text {hmax }}=2000 \mathrm{~Hz}$ is significantly different from the others, mainly between $2000 \mathrm{~Hz}$ and $4000 \mathrm{~Hz}$, as expected.

\section{B.2 Number of body modes}

In order to analyse the influence of the number of body modes, denoted by $N_{b}$, the maximum frequency for the string harmonics is fixed at $f_{\text {hmax }}=10000 \mathrm{~Hz}$, and simulations are performed for $N_{b}=6,20$ and 36, including respectively modes between $0 \mathrm{~Hz}$ and $500 \mathrm{~Hz}, 0 \mathrm{~Hz}$ and $1000 \mathrm{~Hz}$, and $0 \mathrm{~Hz}$ and $1500 \mathrm{~Hz}$. As can be seen in Figures ??a and ??b, the number of body modes strongly influences the string decay since more damping is added as $N_{b}$ increases, leading to a shorter decay. The contribution of the body modes for each case can be seen in the spectra shown in Figure ??c, ??d and ??e. The body 


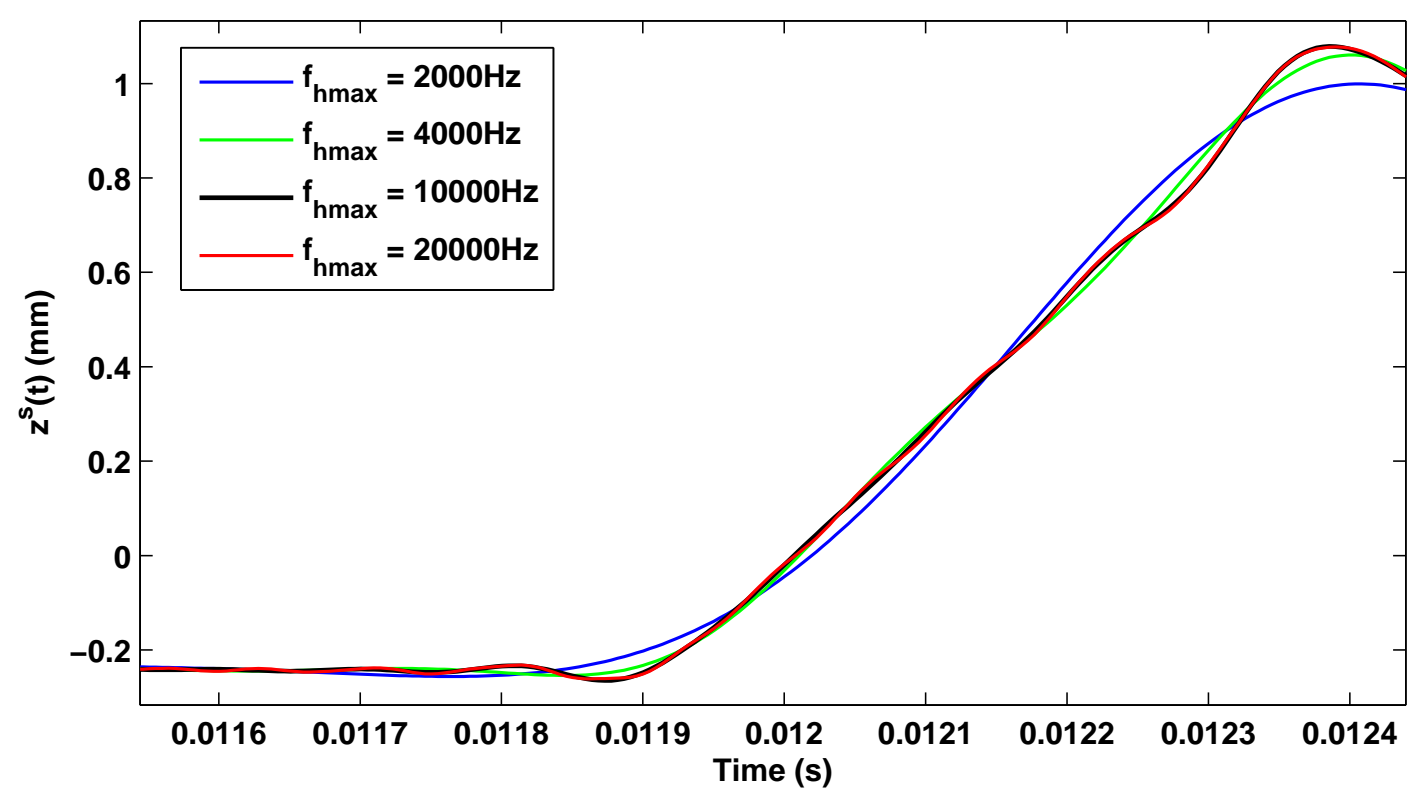

Figure B.1: Comparison of simulated displacements obtained using four different values of maximum frequency $f_{\text {hmax }}(2000 \mathrm{~Hz}, 4000 \mathrm{~Hz}, 10000 \mathrm{~Hz}$ and $20000 \mathrm{~Hz})$ for the string harmonics. One-polarization model of a single string is considered. Zoomed displacement of string 1 (D4) plucked at $8.5 \mathrm{~cm}$ from the bridge, normal to the soundboard plane.

contributions are characterized by inharmonic peaks and in general are less significant than the string contributions represented by the quasi-harmonic peaks. The main spectral differences of the green curve $\left(N_{b}=20\right)$ in comparison to the blue one $\left(N_{b}=6\right)$ are indicated by the arrows in Figure ??d, as well as the main difference of the red curve $\left(N_{b}=36\right)$ in comparison to the green one $\left(N_{b}=20\right)$, indicated in Figure ??e.

\section{B.3 Conclusion}

The analyses above-presented allow us to justify the choices made for simulations in Section ??: $N_{b}=20$, including body modes from $0 \mathrm{~Hz}$ to $1000 \mathrm{~Hz}$, and $f_{h \max }=5000 \mathrm{~Hz}$, which leads to the numbers of string modes $N_{(s)}$ selected and presented in Table ??. These choices were also based on the computational time analysis for different combinations of the parameters $N_{b}$ and $f_{\text {hmax }}$. It is also important to emphasize that "informal" perceptual tests including a small group of musicians supported the choice of the parameters. 

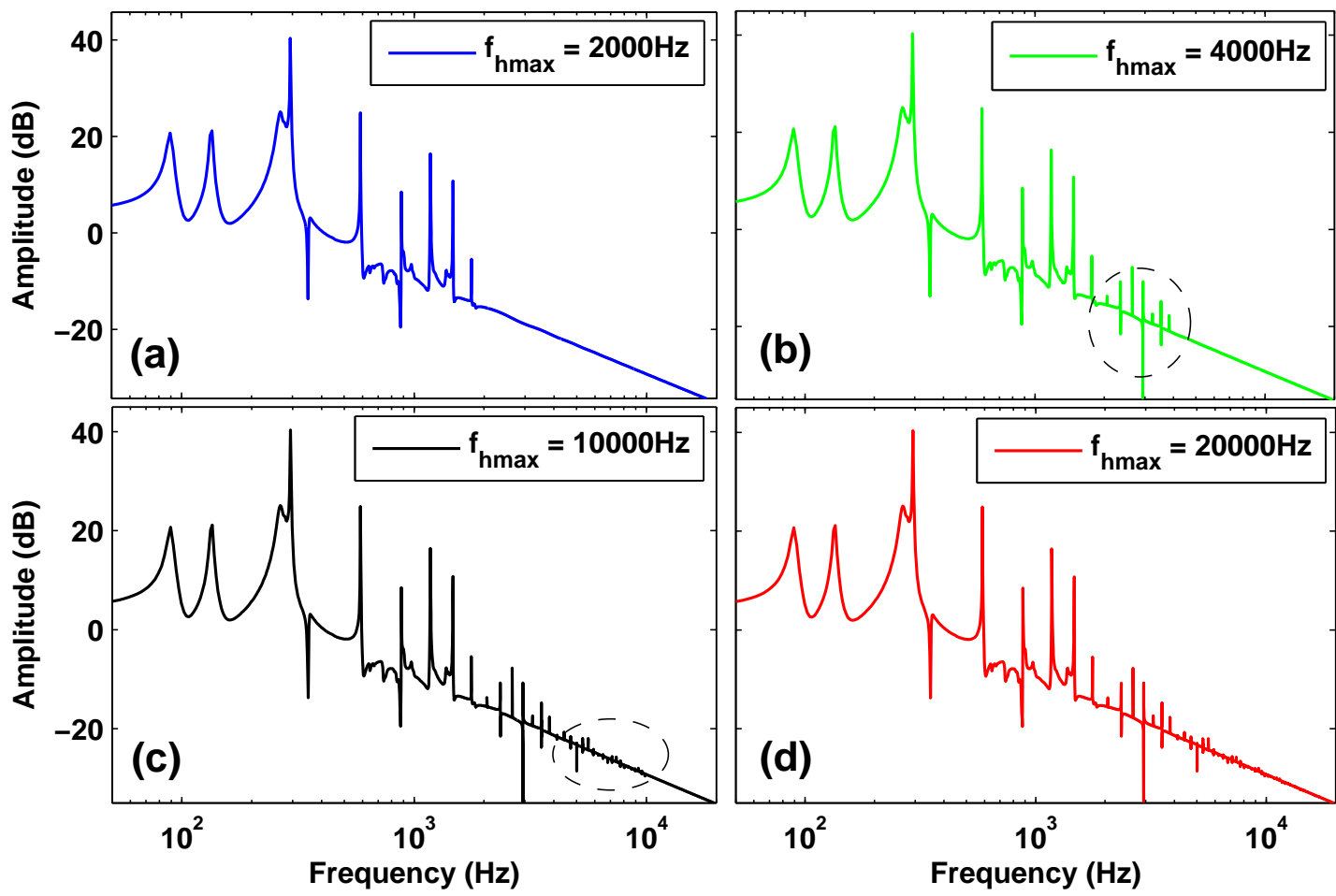

Figure B.2: Comparison of spectra obtained using four different values of maximum frequency $f_{\text {hmax }}$ (2000 $\mathrm{Hz}, 4000 \mathrm{~Hz}, 10000 \mathrm{~Hz}$ and $20000 \mathrm{~Hz}$ ) for the string harmonics. One-polarization model of a single string is considered. Zoomed displacement of string 1 (D4) plucked at $8.5 \mathrm{~cm}$ from the bridge, normal to the soundboard plane. 

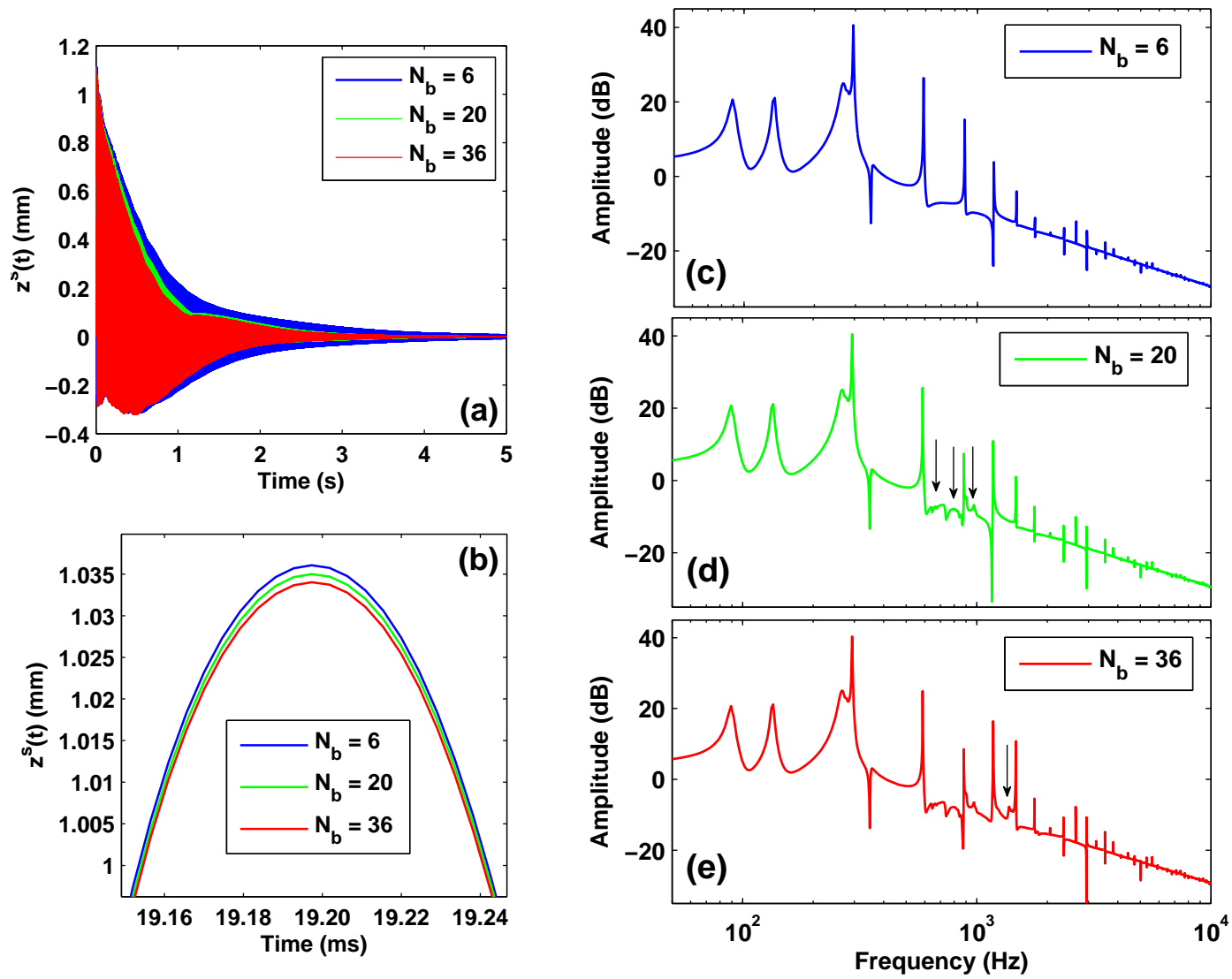

Figure B.3: Comparison of time-histories and spectra obtained using three different numbers of body modes. One-polarization model of a single string is considered. Comparison of (a) global and (b) zoomed displacement time-histories; spectra obtained from the first $300 \mathrm{~ms}$ of displacement for (c) $N_{b}=6(0 \mathrm{~Hz}-500$ $\mathrm{Hz})$, (d) $N_{b}=20(0 \mathrm{~Hz}-1000 \mathrm{~Hz})$ and (e) $N_{b}=36(0 \mathrm{~Hz}-1500 \mathrm{~Hz})$. String 1 (D4) is plucked at $8.5 \mathrm{~cm}$ from the bridge, normal to the soundboard plane. 


\section{Appendix C}

\section{VIBROACOUSTIC FINITE ELEMENT MODELLING OF A viola caipira RESONANCE BOX}

This appendix contains the paper "Modelling Fluid-Structure Interaction in a Brazilian Guitar Resonance Box", published in the proceeding of the $22^{\text {nd }}$ International Congress of Mechanical Engineering (COBEM 2013) in Brazil. A vibroacoustic finite element model of the viola caipira resonance box including the air cavity is presented. Natural frequencies and mode shapes are calculated by means of a numerical modal analysis and compared with with experimental results. 


\title{
MODELING FLUID-STRUCTURE INTERACTION IN A BRAZILIAN GUITAR RESONANCE BOX
}

\author{
Guilherme Orelli Paiva ${ }^{1,2}$; \\ José Maria Campos Dos Santos ${ }^{1}$; \\ ${ }^{1}$ University of Campinas, Rua Mendeleyev, 200 - Cidade Universitária “Zeferino Vaz”, CEP 13083-860, Campinas, \\ SP - Brazil. \\ ${ }^{2}$ E-mail: pitupaiva@fem.unicamp.br
}

Abstract. The relationship between measurable physical properties of a musical instrument and the subjective evaluation of their sound quality is an important subject of musical acoustics research. Therefore, new analytical or numerical methods to predict accurately its vibroacoustic behavior will enable the determination of key parameters that can be used to control instrument tone and sound quality. This work uses theoretical modal analysis with finite element method (FEM) to determine the dynamic behavior of a Brazilian guitar resonance box in terms of modal parameters. A three-dimensional numeric vibroacoustic model of a Brazilian guitar resonance box is implemented in ANSYS FEM software, including all physical components and the orthotropic property of woods. Two vibroacoustic numeric modal analysis are performed. For the first one, a very simple sound hole boundary condition is modeled with no mass load or radiation impedance, i.e., zero pressure at the location of sound hole. For the second one, the effect of the radiation through the sound hole is simulated including a radiation mass load in the sound hole. In both analysis free boundary condition for the resonance box structure is used. The viboracoustic simulated results are compared to each other, and with the experimental test results obtained from an actual Brazilian guitar.

Keywords: vibroacoustic; Brazilian guitar; musical instruments; modal analysis.

\section{INTRODUCTION}

The use of numerical models to determine the dynamic behavior of a musical instrument is a common way to study the relation between its physical properties and sound quality (Wright, 1997; Fletcher and Rossing, 2005; Hurtado et al, 2012). In other words, the determination of objective parameters may be used to obtain and control subjective requirements like performance and sound quality. In this sense, many works has presented methods for analytical and numerical prediction of the vibroacoustic behavior of string instruments With the advent of technology and consequent improvement of the computational processing, numerical models have been used to simulate complex systems and calculate modal parameters like vibration modes and natural frequencies, which are determinant in the tone and sound power desired for a musical instrument (Broke, 1992). Therefore, the use of these simulation tools seems to be valuable for the musical instrument design. By varying the structural and acoustic parameters it is possible to obtain different modal parameters without need for constructing multiple prototypes. This process can help the instrument makers to improve instrument design by avoiding empirical trial and error methods.

This paper presents finite element method (FEM) modal analysis to determine the vibroacoustic behavior of Brazilian guitar resonance box. Modal analysis technique allows to find out the natural frequencies and the corresponding mode shapes of structural, acoustic and vibroacoustic (fluid and structure coupled) systems. It is well known that both air cavity and the wood plates of resonance box act as elastic elements and interact each other in the coupled system, generating their vibroacoustic modes. Like many string instruments, the Brazilian guitar resonance box have its air cavity connected to the outside by a hole, which characterize the system as Helmholtz resonator. Hence its dynamic behavior interact with the exterior environment. The lowest air cavity mode, which is not consider to be coupled with the structure, is commonly called Helmholtz resonance and named as A0. The higher modes of the air cavity (named as A1, A2,..) comes from cavity's stationary waves and are not harmonically related to A0 (Elejabarrieta et al, 2002). These modes have been studied by several authors for guitar and violin resonance boxes and their influence on the instrument has been demonstrated (Jansson, 1977; Firth, 1977; Roberts, 1997).

Numerical simulations by FEM have been previously applied to obtain modal parameters of string instruments (Elejabarrieta et al, 2002a; Curtu et al, 2005). The authors were the firsts to apply it for the Brazilian guitar resonance box (Paiva and Santos, 2012). However, in this paper the sound hole boundary condition was modeled with zero pressure (no mass load or radiation impedance). This assumption generates imprecision for the Helmholtz resonance and for the higher modes (Elejabarrieta $e t$ al, 2002). In this work the radiation effect through the sound hole is simulated by incrementing the air neck length in the hole (Kinsler et al, 1982). The simulated results are compared with that of previous work and the differences are discussed. In order to validate the numerical simulations, the results of vibroacoustic FEM model are compared with that obtained by experimental test. 


\section{THE BRAZILIAN GUITAR}

The Brazilian guitar is a countryside musical instrument. It presents different characteristics that vary regionally, by configuring as a sparse group of string musical instruments. The instrument diversity comes from different geometric shapes, number of strings, wood types and the tuning types. The expression "Brazilian Guitar" is capable to qualify the instrument in all its variations. So many names and singularities for these guitars can be found along the Brazilian territory. This paper is focused on viola Caipira, which is the most known and played in all regions of Brazil, particularly in the Southeast and Midwest regions. Generally, the viola Caipira has 10 strings combined at five pairs. Two pairs are tuned in high notes on the same fundamental frequencies, i.e., the same note at the same frequency (unison), while the remaining pairs are tuned to the same note, but with a difference of one octave in their frequency (rate 2:1).

The viola Caipira is derived from the Portuguese guitar, which originates from Arabic instruments like lutes. In the fifteenth and sixteenth centuries, the Portuguese guitar was widespread in Portugal, being considered the main instrument of the minstrels and troubadours. It arrived in Brazil through the Portuguese settlers from different regions and has passed to be used by the Jesuits in the Indian catechesis (Vilela, 2004). Subsequently, the natives began to build rudimentarily these guitars using woods from Brazil. This instrument can be regarded as the forerunner of the viola Caipira we know today. At the beginning the viola Caipira practically kept the basic structure of its ancestor, following the same pattern.

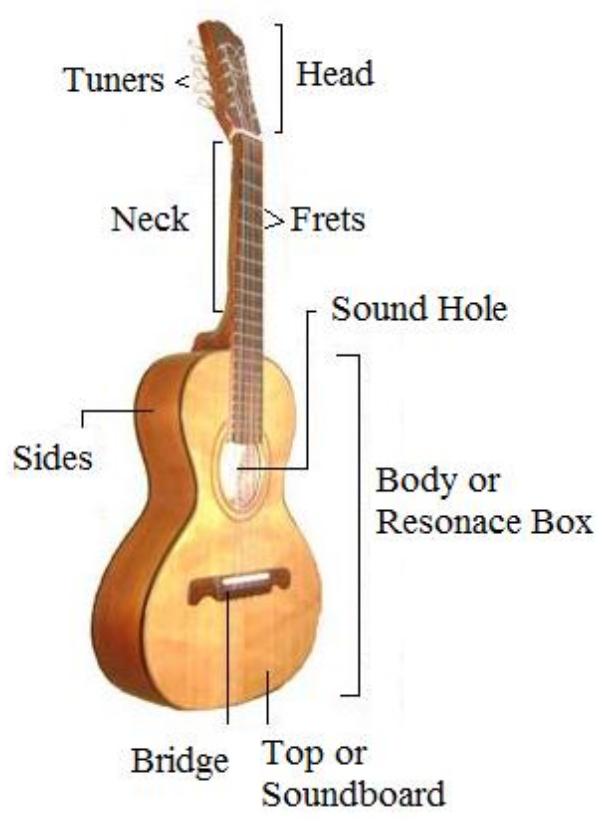

(a)

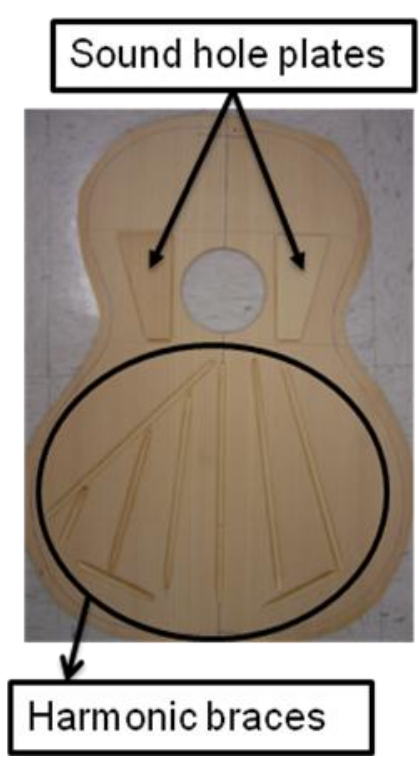

(b)

Figure 1. Main parts of a Brazilian guitar (viola Caipira): (a) External; and (b) Internal.

The main parts of a viola Caipira are similar to a classic guitar, as shown in Fig. 1. It is important that the strings are always adjusted to the proper tension, i.e., always respecting the structural capacity of the instrument, specially the soundboard and head, which are under constant compressive loading caused by the tension resulting in the tuners and bridge. There are also dynamic loads that are present while the instrument is played, and forces generated by gradients of temperature and humidity. Therefore, it is important to pay attention to the structural details when designing and building an instrument. The resonance box (or body) is composed by the top plate, back plate, sides and internal structures. These parts enclose the acoustic cavity, which communicates with the external air through the hole of the instrument (sound hole). The strings are attached to the soundboard through the bridge (Fig. 1a). The internal fixings and reinforcements (sound hole plates; harmonic braces; braces; lining, neck and tail blocks) are shown in Fig. 1 b.

The dynamic behavior of a Brazilian guitar is determined by the interaction of many components that radiates sound in different ways. This is because sound behaves differently depending on the wave length of the sound compared to the dimension of the radiator. Rossing (1988) proposed the scheme shown in Fig. 2, which presents two different ways for high and low frequencies sound radiates and propagates in a guitar. 


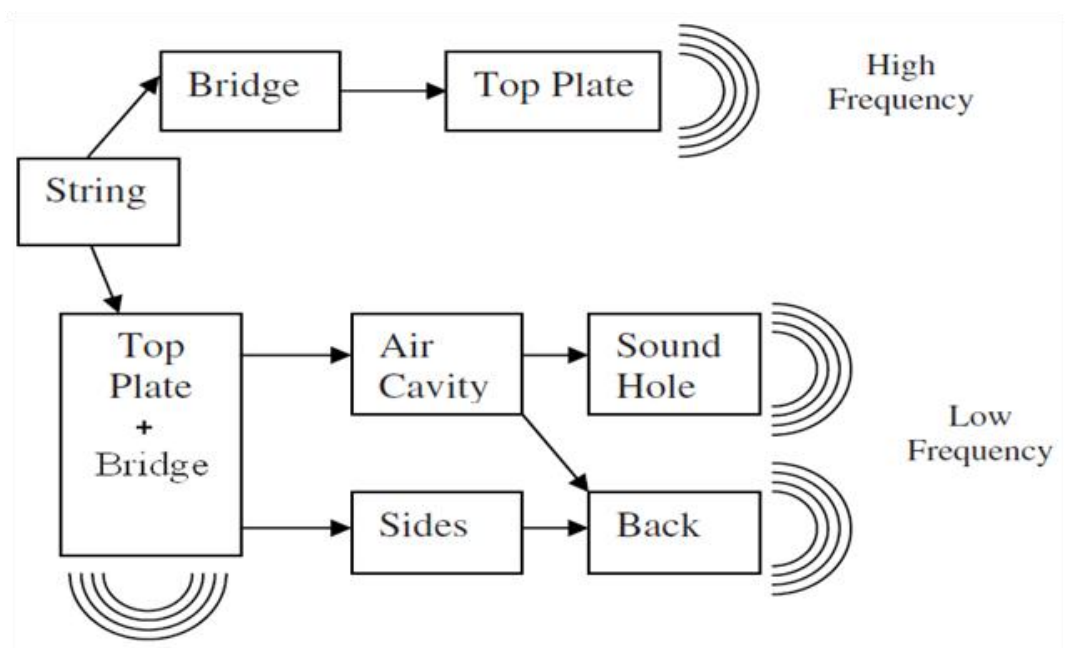

Figure 2. Schematic of radiation and energy flow in a guitar. (adapted from Rossing, 1988)

The production of sound starts in the interaction between the player's fingers and the strings. When a string is plucked, its vibration can be described in terms of transversal modes. The corresponding frequencies have an almostharmonic relationship, and changing the plucked position the player excites different string modes and vary the tone. , At high frequencies the strings radiate a little part of energy through the air, while the most part of energy is transmitted to soundboard via bridge. In the other hand, at low frequencies the bridge and the soundboard behave as a unique structure, being excited directly by the strings.

\section{VIBROACOUSTIC MODAL ANALYSIS}

\subsection{Finite element method: theorical basis}

This section presents a brief mathematical formulation of the finite element method for the vibroacoustic (fluidstructure interaction) modal analysis. For the coupled field it is needed to take into account the effects of fluid-structure interaction. The formulation presented here was developed by Zienkiewicz and Newton (1969). Based on the elasticity theory, the dynamic behavior of a linear elastic solid (small deformations) can be written in index notation as:

$$
\sigma_{i j, j}+f_{i}=\rho_{s} \ddot{u}_{i}
$$

where $\sigma_{i j}$ is the stress tensor, $f_{i}$ is the body forces vector, $\rho_{s}$ is the solid density, $u_{i}$ is the displacement, and $i$ and $j=x, y$, $z$. The effect of fluid over the solid is included in the interfaces trough the fluid pressure over the solid surface, i.e., the balance of forces in the normal direction to the field interfaces must be imposed as

$$
\sigma_{i j} n_{i}=n_{i} p
$$

where $n_{i}$ is the interface normal vector and $p$ is the acoustic pressure. The acoustic wave equation can be written as:

$$
\nabla^{2} p+\frac{1}{c_{0}^{2}} \ddot{p}=-g
$$

where $c_{0}$ is the sound speed and $g$ is the source field. The effect of the solid over the fluid is also considered in the domain interfaces through the kinematic compatibility of the solid in contact with the fluid, namely

$$
\frac{\partial p}{\partial n}=-\rho_{0} \ddot{u}_{n}
$$

where $\rho_{0}$ is the fluid density and $u_{n}$ is the displacement component normal to the interface. Applying the weighted residual method and discretizing with finite elements on Eqs. (1) to (4), we obtain the element mass matrix, element stiffness matrix, element volumetric stiffness matrix, element compressibility matrix and element interface matrix as: 
Paiva, G. O. and Dos Santos, J. M. C.

Modeling Fluid-Structure Interaction in a Brazilian Guitar Resonance Box

$$
\mathbf{M}^{e}=\int_{\Omega} \rho_{s} \mathbf{N}_{s}^{T} \mathbf{N}_{s} d \Omega ; \mathbf{K}^{e}=\int_{\Omega} \mathbf{B}_{s}^{T} \mathbf{D} \mathbf{B}_{s} d \Omega ; \mathbf{E}^{e}=\frac{1}{c_{0}^{2}} \int_{\Psi} \rho_{0} \mathbf{N}_{f}^{T} \mathbf{N}_{f} d \Psi ; \mathbf{H}^{e}=\int_{\Psi} \mathbf{B}_{f}^{T} \mathbf{B}_{f} d \Psi ; \mathbf{L}^{e^{T}}=\int_{\Gamma_{i}} \mathbf{N}_{f}^{T} \mathbf{n} \mathbf{N}_{s} d \Gamma_{i},
$$

where $\mathbf{N}$ is the element shape function matrix, $\mathbf{B}$ is the nodal strain-displacement matrix, $\mathbf{D}$ is the constitutive law matrix and the index ${ }_{S}$ and ${ }_{f}$ refers to solid and fluid domain, respectively. The Greek letters $\Omega, \Psi$ and $\Gamma$ refers to the geometry of the structural, fluid and interface domain, respectively. Writing the equations to the coupled system in terms of a global matrix in the frequency domain and considering the free vibration condition we have:

$$
\left(\left[\begin{array}{cc}
\mathbf{K} & -\mathbf{L} \\
\mathbf{0} & \mathbf{H}
\end{array}\right]-\boldsymbol{\Lambda}_{a}\left[\begin{array}{cc}
\mathbf{M} & \mathbf{0} \\
\rho_{0} \mathbf{L}^{T} & \mathbf{E}
\end{array}\right]\right)\left\{\begin{array}{l}
\mathbf{d} \\
\mathbf{p}
\end{array}\right\}=\left\{\begin{array}{l}
\mathbf{0} \\
\mathbf{0}
\end{array}\right\}
$$

where $\Lambda_{a}$ is a diagonal matrix of the square of the natural frequency of coupled domain and $\{\mathbf{d} \mathbf{p}\}^{\mathrm{T}}$ is the displacementacoustic pressure nodal vectors of the corresponding vibration mode shapes. This solution is also known as vibroacoustic modal analysis.

\subsection{Resonance box numerical model}

The finite element computer model geometry of the resonance box was built in the software ANSYS 13.0, using the parts and dimensions of a commercial viola Caipira, brand Rozini, Ponteio Profissional model (Rozini Instrumentos Musicais. http://www.rozini.com.br/Defaultb.asp). Figure 3 shows the guitar main dimensions and the components included in the Finite Element (FE) model.

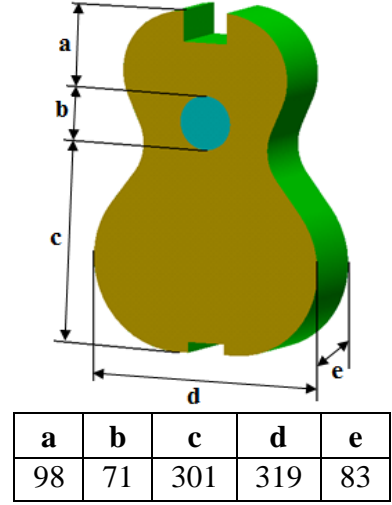

(a) Dimension in mm.

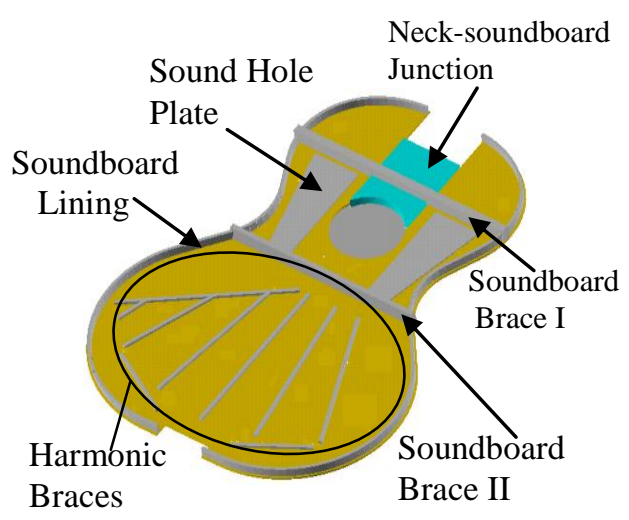

(b) Soundboard

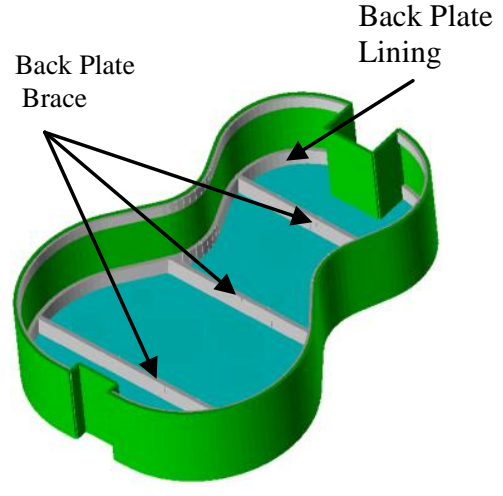

(c) Back Plate and Sides

Figure 3. Rozini Brazilian guitar resonance box: (a) Main dimensions. (b) (c) Components included in FE model.

The difficulty to identify the wood of resonance box components led to the choices by indications from the literature (Bergman et al, 2010). Furthermore, when it was possible to identify the wood component, was not found its mechanical properties, leading to the use of a similar timber properties. Thus, it is assumed that the soundboard and internal reinforcements are made from Sitka Spruce (Picea sitchensis), and the back plate, sides and neck-soundboard junction are made of Yellow Birch (Betula alleghaniensis). Table 1 shows the mechanical properties of woods used in FE model. The thicknesses of soundboard, back plate and sides are $3.0 \mathrm{~mm}, 3.5 \mathrm{~mm}$ and $2.0 \mathrm{~mm}$, respectively. Table 2 shows the cross section dimensions of braces and linings included in FE model.

The vibroacoustic ANSYS model assumes the resonance box cavity filled with air. The air is modeled with fluid element (FLUID30) and the structures are modeled with plate element (SHELL63) and beam element (BEAM188).

Table 1. Mechanical properties of woods (Bergman et al, 2010).

\begin{tabular}{|c|c|c|c|c|c|c|c|c|c|c|}
\hline Wood & $\begin{array}{c}E_{x} \\
{[\mathrm{MPa}]}\end{array}$ & $\begin{array}{c}E_{y} \\
{[\mathrm{MPa}]}\end{array}$ & $\begin{array}{c}E_{z} \\
{[\mathrm{MPa}]}\end{array}$ & $\begin{array}{c}G_{x z} \\
{[\mathrm{MPa}]}\end{array}$ & $\begin{array}{c}G_{x y} \\
{[\mathrm{MPa}]}\end{array}$ & $\begin{array}{c}G_{y z} \\
{[\mathrm{MPa}]}\end{array}$ & $v_{x z}$ & $v_{x y}$ & $v_{z y}$ & $\begin{array}{c}\rho \\
{\left[\mathrm{Kg} / \mathrm{m}^{3}\right]}\end{array}$ \\
\hline Spruce & 10.340 & 800 & 440 & 660 & 630 & 30 & 0.372 & 0.467 & 0.435 & 460 \\
\hline Birch & 11.320 & 880 & 560 & 830 & 760 & 190 & 0.426 & 0.451 & 0.697 & 668 \\
\hline
\end{tabular}


Table 2. Cross section dimensions of braces and linings.

\begin{tabular}{|c|c|c|c|c|c|c|c|}
\hline $\begin{array}{c}\text { Cross } \\
\text { Section }\end{array}$ & $\begin{array}{c}\text { Dimensions } \\
{[\mathbf{m m}]}\end{array}$ & $\begin{array}{c}\text { Harmonic } \\
\text { Brace }\end{array}$ & $\begin{array}{c}\text { Soundboard } \\
\text { Brace I }\end{array}$ & $\begin{array}{c}\text { Soundboard } \\
\text { Brace II }\end{array}$ & $\begin{array}{c}\text { Back Plate } \\
\text { Brace }\end{array}$ & $\begin{array}{c}\text { Soundboard } \\
\text { Lining }\end{array}$ & $\begin{array}{c}\text { Back Plate } \\
\text { Lining }\end{array}$ \\
\hline \multirow{3}{*}{$\mathbf{b} \sqrt{\mathbf{a}}$} & $\mathbf{a}$ & 3.5 & 8.0 & 9.0 & 9.0 & 3.0 & 4.5 \\
\cline { 2 - 9 } & $\mathbf{b}$ & 3.5 & 18.0 & 5.0 & 5.0 & 12.0 & 16.0 \\
\cline { 2 - 9 } & $\mathbf{c}$ & 3.5 & 8.0 & 16.0 & 16.0 & 3.0 & 4.5 \\
\hline
\end{tabular}

Fluid-structure interaction is obtained with the fluid-structure coupling matrix (FSI), which conduct to a solution of an unsymmetrical eigenvalue problem. A mapped mesh is constructed in solid and fluid domains, which contains a total number of 23,429 elements and 20,109 nodes. Two simulated vibroacoustic modal analysis are performed using different boundary conditions in the sound hole. First one is the simplest which assumes air radiation impedance at sound hole equal to zero, i.e., null pressure at the location of sound hole. This causes loss of accuracy for the Helmholtz resonance and for several of the higher modes. Second one considers that the effect of the radiation through the sound hole can be approximated as a Helmholtz resonator (Figure 4a), which assumes that the moving fluid on the neck radiates sound into the surrounding medium like an open-ended pipe. In order to simulate the effect of radiation in low frequencies through the sound hole, some works have modeled the cavities of string instruments as a Helmholtz resonator (Elejabarrieta et al, 2002b; Bretos et al,1999; Cremer, 1984).

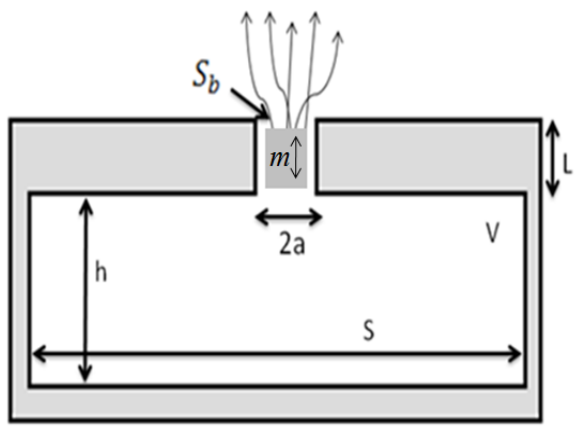

(a)

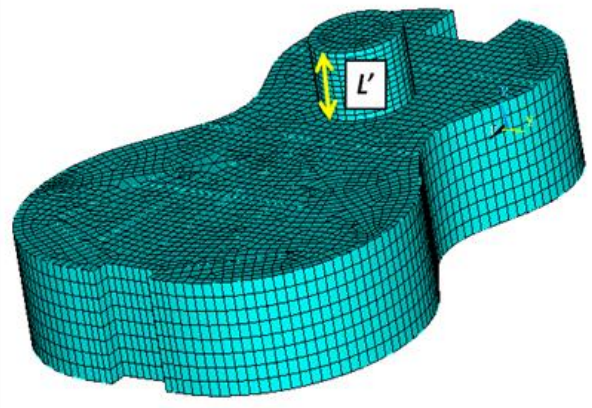

(b)

Figure 4. (a) Geometric parameters of a Helmholtz resonator. (b) Fluid finite element mesh including the neck effective length.

The neck of Helmholtz resonator radiates sound, providing radiation resistance and radiation mass. At low frequencies a circular opening of radius $a$ is loaded with a radiation mass equal to that of the fluid contained in a cylinder of area $\pi a^{2}$ and length $\Delta L$. By taking in account the outer and inner opening of the neck and assuming that they are equivalent to a pipe flanged termination the total effective mass is given by (Kinsler et al, 1982):

$$
m=\rho_{0} S_{b} L^{\prime},
$$

where $S_{b}$ is the neck cross section area, and $L^{\prime}=L+\Delta L$ is the effective neck length. An opening consisting of a circular hole in the thin wall of a resonator, as the sound hole in the resonance box, will have an effective length of (Kinsler et al, 1982),

$$
L^{\prime}=1,6 a \text {. }
$$

By applying this concept to the FEM model at the sound hole as a boundary condition, the effective neck length is calculated (Eq. 8) and a corresponding air cylinder with the volume $V$, is included into the finite element geometry. Table 3 shows the calculated values of Helmholtz resonator parameters. Also, null pressure at the top surface and rigid walls at lateral surface of the air neck cylinder are applied. Figure $4 \mathrm{~b}$ shows the fluid finite element mesh including the air neck cylinder with the effective length. A vibroacoustic modal analysis is performed, where the free boundary condition was applied to the structure.

Table 3. Helmholtz resonator parameters.

\begin{tabular}{|c|c|c|c|c|c|c|c|}
\hline$\rho_{0}\left[\mathrm{Kg} / \mathrm{m}^{3}\right]$ & $m[\mathrm{Kg}]$ & $S_{b} \cdot\left[\mathrm{m}^{2}\right]$ & $L^{\prime}[\mathrm{m}]$ & $L[\mathrm{~m}]$ & $a[\mathrm{~m}]$ & $c_{0}[\mathrm{~m} / \mathrm{s}]$ & $V\left[\mathrm{~m}^{3}\right]$ \\
\hline 1.125 & $2.0 \times 10^{-4}$ & $2.8 \times 10^{-3}$ & $4.8 \times 10^{-2}$ & $3.0 \times 10^{-3}$ & $3.0 \times 10^{-2}$ & 343 & $0.85 \times 10^{-2}$ \\
\hline
\end{tabular}


Paiva, G. O. and Dos Santos, J. M. C.

Modeling Fluid-Structure Interaction in a Brazilian Guitar Resonance Box

Table 4 shows the comparison between the first 5 natural frequencies and mode shapes of the numeric model using the two different boundary conditions, where one will be called Simple BC and the other will be called Helmholtz BC.

Table 4. First 5 vibroacoustic natural frequencies and mode shapes of the guitar resonance box for the two boundary conditions: Simple BC and Helmholtz BC.

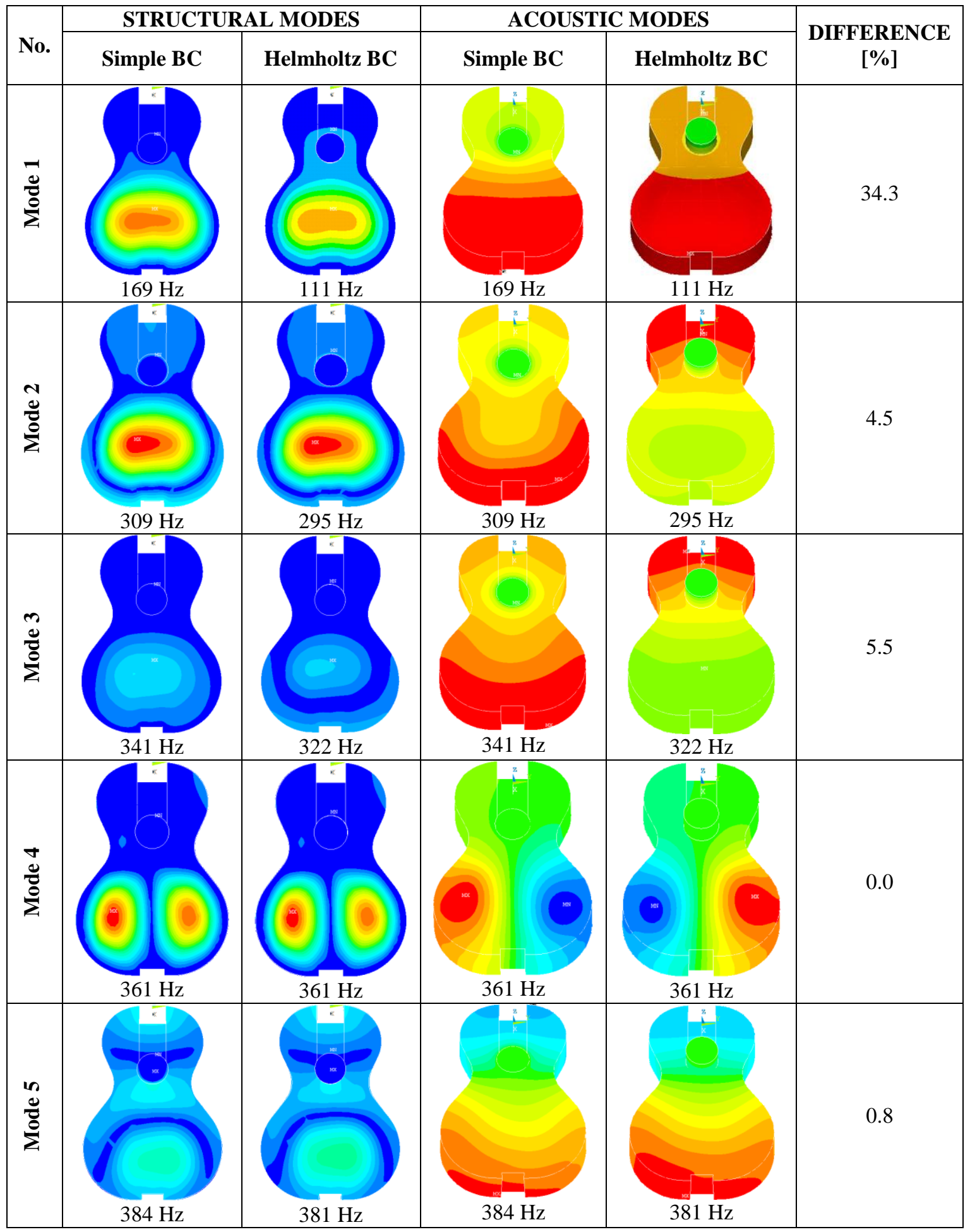

For the structural modes (Tab. 4 and Tab. 6) the blue color indicates nodal regions and red color indicates antinodes regions. For the acoustic modes blue and red colors indicate minimum and maximum antinodes regions, and green color indicates nodal regions. 
From Tab. 4, it can be seen that different boundary condition presents a strong variation in the first natural frequency $(34.3 \%)$, a little bit less in the second $(4.5 \%)$ and third $(5.5 \%)$, and noting or negligible in the fourth and fifth, respectively. The structural mode shape was not significantly affected by the changes in boundary conditions. The modes look almost the same. The main difference was noted in the first acoustic mode shapes. As expected, the largest differences are in the first three lower modes. The fourth and fifth mode shapes are the same, only the phase is reversed for the fourth mode.

To verify this numerical results, experimental test are made in the actual viola. Experimental test was conducted to the 5 first natural frequencies and mode shapes. Test was performed in an anechoic chamber in a complete viola (without strings), instead of the resonance box. By using a sine sweep signal, an acoustic excitation (loudspeaker) is applied to the guitar suspended by elastic bands (free condition). The natural frequencies are obtained by determining the resonance peaks of the soundboard speed (using a laser vibrometer) or acoustic pressure (using a microphone inside the resonance box). The structural mode shapes are obtained using Chladni's figures. To obtain these figures the guitar is excited in each natural frequency, and spreading minced leaves of tea on the soundboard these will accumulate on the regions of nodal lines (zero speed) revealing the mode shape. Figure 5 shows the arrangement of experimental setup and a Chladni figure.

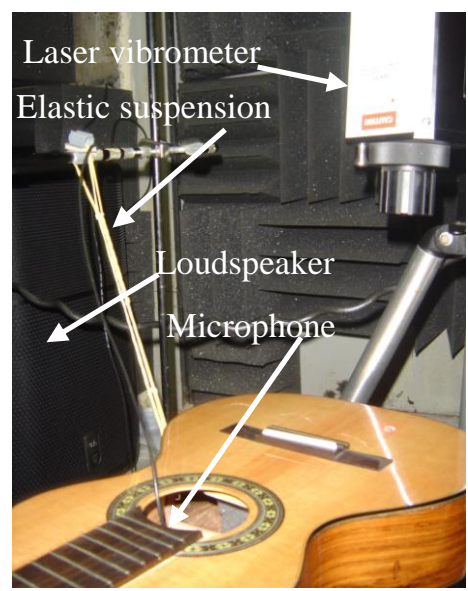

(a)

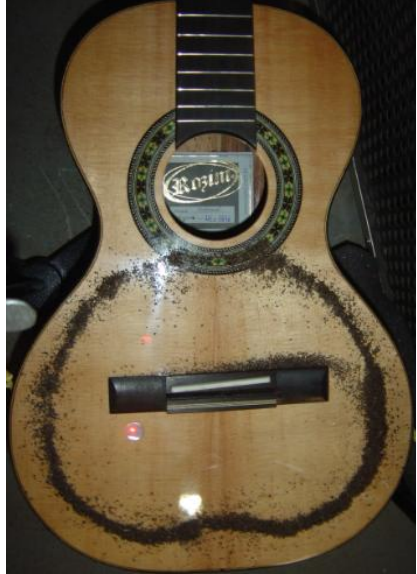

(b)

Figure 5 - Brazilian guitar: (a) experimental setup; and (b) Chladni figure.

Table 5 presents the first 5 structural natural frequencies and mode shapes obtained with the numerical simulations (Simple and Helmholtz BC's) and the experimental measurements on the actual viola. It can be observed that Helmholtz BC can improve the simulation results for natural frequencies as compared to Simple BC. The relative error to the experimental result is reduced from $25.2 \%$ (Simple BC) to $17.5 \%$ (Helmholtz BC) in the first mode. Also, there is significant error reduction in natural frequency for the second and third modes. Only for the two last modes these reduction is lees noteworthy. These results corroborate the theory about Helmholtz BC applied here, which correct only the lower natural frequencies.

Table 5. Comparison of the first 5 natural frequencies obtained by the experimental and the numerical models.

\begin{tabular}{|c|c|c|c|c|c|}
\cline { 2 - 6 } \multicolumn{1}{c|}{} & \multirow{2}{*}{ Experimental } & \multicolumn{2}{c|}{ Simple BC } & \multicolumn{2}{c|}{ Helmholtz BC } \\
\hline Mode & Frequency (Hz) & Frequency (Hz) & Error (\%) & Frequency (Hz) & Error (\%) \\
\hline $\mathbf{1}$ & 135 & 169 & 25.2 & 111 & 17.5 \\
\hline $\mathbf{2}$ & 266 & 309 & 13.9 & 295 & 9.8 \\
\hline $\mathbf{3}$ & 321 & 341 & 5.8 & 322 & 0.3 \\
\hline $\mathbf{4}$ & 340 & 361 & 5.8 & 361 & 5.8 \\
\hline $\mathbf{5}$ & 374 & 384 & 2.6 & 381 & 1.8 \\
\hline
\end{tabular}

Table 6 presents a comparison of the first 5 natural frequencies and mode shapes between the numerical Helmholtz $\mathrm{BC}$ and experimental results.

The obtained Chladni figures show that only the experimental modes with enough power to push the tea particles to nodal lines can be revealed. From the Tab. 6 it can be seen that only the two first modes present experimental mode shapes for both: the soundboard and the back plate. The numerical results for these (modes 1 and 2) are in good agreement with the experimental. Nevertheless, for the others experimental modes (modes 3, 4 and 5) only one mode shape, soundboard or back plate was identified. Experimental modes 3 and 5 occur on the back plate, while 
Paiva, G. O. and Dos Santos, J. M. C.

Modeling Fluid-Structure Interaction in a Brazilian Guitar Resonance Box

experimental mode 4 occurs on soundboard. All of them present good agreement with the numerical ones. From the experimental results presented it is observed that there are modes that have not found due to limitations of the experimental technique. This requires great contrasts between regions of significant displacement and regions of zero displacement. Moreover, the numerical results are more close to the experimental than that obtained previously by the authors (Paiva and Santos, 2012), which do not consider the Helmholtz boundary condition.

Table 6. First 5 natural frequencies and mode shapes of the guitar resonance box using numerical (Helmholtz BC) and experimental models.

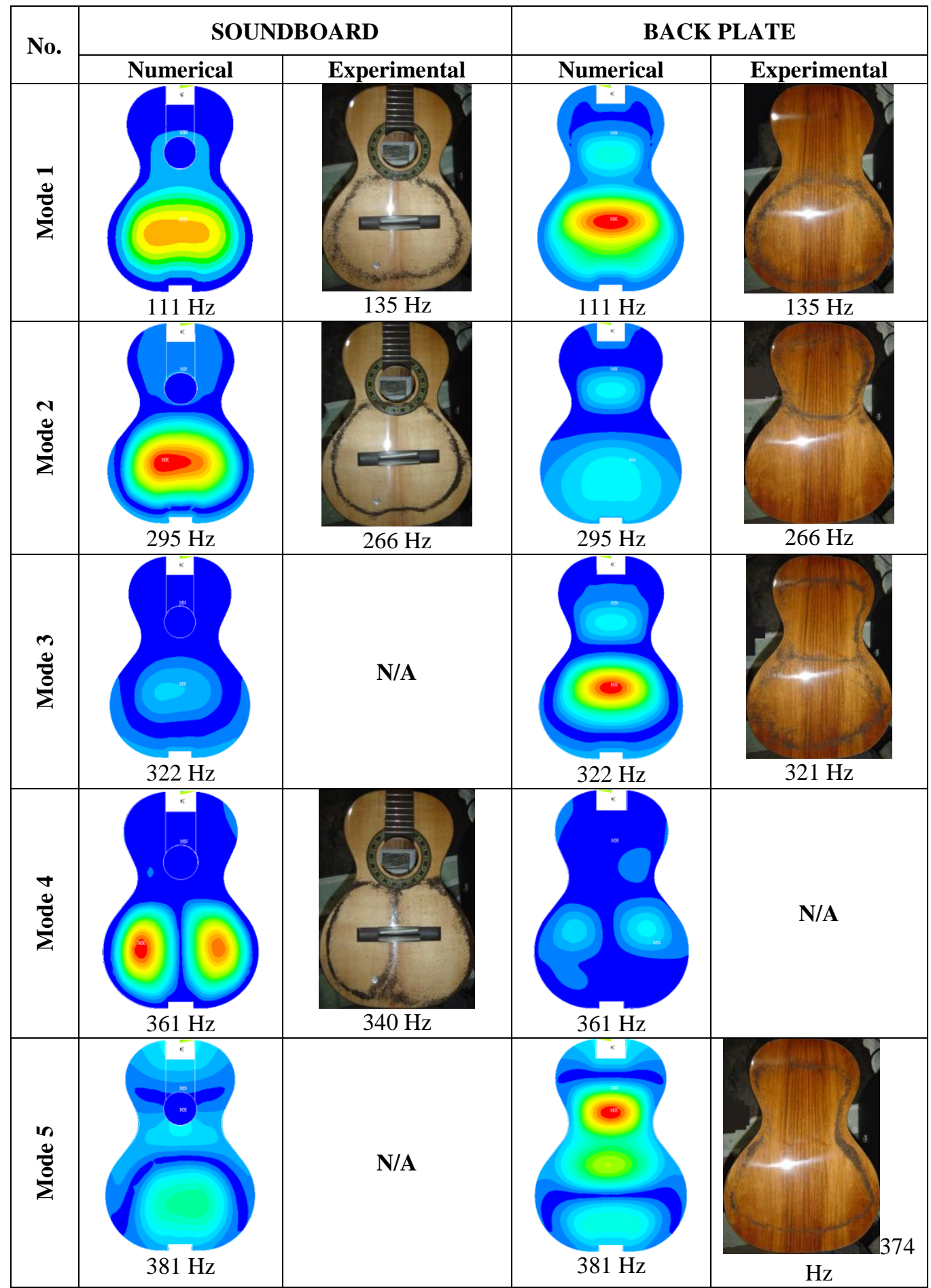

The disagreement between experimental and simulated results can also be attributed to others simplifications assumed in the computer model. Some wood components of the resonance box had their mechanical properties substituted by that of similar wood. Also, the lacquer layer, the presence of the neck and the influence of external fluid 
were not considered in the computational model. Therefore, the comparative results in Tab. 5 and 6 confirm the improvement of vibroacoustic model with the Helmholtz boundary condition.

\section{CONCLUSION}

The Brazilian guitar was briefly described with regard to its historical, structural and acoustical characteristics. A procedure for vibroacoustic modal analysis by finite element method with ANSYS software was developed. Two simulated vibroacoustic modal analysis are performed using different boundary conditions in the resonance box sound hole. The simplest assumes air radiation impedance at the resonance box sound hole equal to zero. This causes loss of accuracy for the Helmholtz resonance and for several of the higher modes. The other considers that the effect of the radiation through the sound hole can be approximated as a Helmholtz resonator, which assumes that the moving fluid on the neck (sound hole) radiates sound into the surrounding medium like an open-ended pipe. Additionally, to validate the numerical results, experimental test was made in the actual Brazilian guitar using Chladni figures technique. From the simulated results it is clear the improvement for the lower frequency modes using de neck length correction at the sound-hole as compared with the experimental one. As expected, the largest differences were noted in the natural frequencies, particularly for the three first modes (lower frequencies). The fourth and fifth modes are not affected significantly in terms of natural frequency. The corresponding shapes of the acoustic modes are influenced in the same direction, i.e., for the three first modes the changes are significant, while the others do not change significantly. The shapes of structural modes do not seem to change notably. The disagreement between experimental and simulated results can also be attributed to others simplifications assumed in the computer model. Mechanical properties of some wood components of the resonance box were substituted by that of similar wood. Also, the lacquer layer, the presence of the neck and the influence of external fluid were not considered in the computational model. Therefore, the comparative results confirm some improvement of vibroacoustic model with the Helmholtz BC as compared to the Simple BC.

In general, it was observed that the finite element method proved to be effective and can determine the dynamic behavior of the resonance box of the guitar. To obtain better results it is important to indentify all the actual woods used and its mechanical properties. Experimental technique of Chladni figures presents certain limitations to identify modes with low energy content. This suggests to carrying out an experimental modal analysis, which is more complete and efficient. Furthermore, it must be observed that the determination of the modal characteristics of the resonance box is only an initial stage about the study to obtain tones and sound power desired for stringed instruments. But, this information is a helpful tool for luthiers and manufactures to get more control over the quality of the instrument in different stages of its construction. Finally, to go further in this research we need additional tests and psychoacoustic measurements of the sound field, which explain subjective evaluations and allow their relationship with objective parameters.

\section{ACKNOWLEDGEMENTS}

The authors would like to thank to Rozini Instrumentos Musicais, CNPq, Fapesp, and CAPES for supporting this work.

\section{REFERENCES}

Bergman, R., Cai, Z., Carll, C. G., Clausen, C. A., Dietenberger, M. A., Falk, R. H.; Frihart, C. R., Glass, S. V., Hunt, C, G., Ibach, R. E., Kretschmann, D. E., Rammer, D. R., Ross, R. J., Star, N. M., 2010, "Wood Handbook-Wood as na Engineering Material", General Technical Report FPL-GTR-190, Madison, WI: U.S. Department of Agriculture, Forest Service, Forest Products Laboratory, 508 p.

Bretos, J., Santamaria, C., Alonso-Moral, J., 1999, "Vibrational patterns of a violin shaped air cavity obtained finite element modeling", Acustica, 85, 1- 3.

Curtu, I., Stanciu, M. D., Cretu, N. C., Rosca, C. I., 2009, "Modal Analysis of different types of classical guitar bodies", Proceedings of the 10th WSEAS International Conference on ACOUSTICS \& MUSIC: THEORY \& APPLICATIONS, 6 p.

Cremer, L., 1984, “The Physics of the Violin”, Cambridge, MA: The MIT Press.

Dickens, F. T., 1978, "Inertance of the guitar sound hole", Catgut Acoustical Society News Letter, 29, 27-28.

Elejabarrieta, M. J., Ezcurra, A., Santamaría, C., 2002a, "Coupled modes of resonance box of the guitar", J. Acoust. Soc. Am., Vol. 111, No. 5, pp. 2283-2292.

Elejabarrieta, M. J., Santamaria, C., Ezcurra, A., 2002b, “Air Cavity Modes in the Resonance Box of the Guitar: The Effect of Sound Hole" Journal of Sound and Vibration 252(3), 584-590.

Firth, I. M., 1977, "Physics of the guitar at the Helmholtz and first top-plate resonances", J. Acust. Soc. Am., 61, 588193.

Fletcher, N. H. and Rossing, T.D, 2005, “The Physics of Musical Instruments”, Springer, New York, 2005, 2nd ed. 
Paiva, G. O. and Dos Santos, J. M. C.

Modeling Fluid-Structure Interaction in a Brazilian Guitar Resonance Box

Hurtado, E. G., Ortega, J. C., Arreguin, J. M. R., Olmedo, A. S., Meneses, J. P., 2012, "Vibration Analysis in the design and construction of an acoustic guitar", International Journal of Physical Sciences, 7(13), pp. 1986-199.

Jansson, E. V., 1977, “Acoustical properties of complex cavities prediction and measurements of resonance properties of violin-shaped an guitar-shaped cavities", Acustica 37, 211-221.

Kinsler, L. E., Frey, A. R., Coppens, A. B., Sanders, J. V., 1982, "Fundamentals of Acoustics", 4th ed., John Wiley \& Sons, New York, $548 \mathrm{p}$.

Paiva, G. O., Santos, J. M. C. dos, 2012, "Análise Modal Vibroacústica da Caixa de Ressonância de uma Viola Caipira", CONEM2012, São Luis, MA, 2012.

Paiva, G. O., 2013, “Análise Modal Vibroacústica da Caixa de Ressonância de uma Viola Caipira ”, M.Sc. Thesis, State University of Campinas, $103 \mathrm{p}$.

Rossing, T. D., Popp, J. and Polstein, D., 1985, “Acoustic Response of guitars” Proceeding of SMAC'83, Royal Swedish Academy of Music, Stockholm.

Rossing, T. D., 1988, "Sound Radiation from Guitars" American Lutherie, 16.

Roberts, G. W., 1997, "Research papers in violin Acousticsv1975-1993”, (Hutchins, C. M., editor) Acoust. Soc. Am., 635-640.

Wright, H., 1996, "The acoustics and psichoacoustics of the guitar ", Departament of Physics and Astronomy, University of Wales, College of Cardiff, PHD Thesis, 260p.

\section{RESPONSIBILITY NOTICE}

The authors are the only responsible for the printed material included in this paper. 



\section{Appendix D}

\section{NUMERICAL AND EXPERIMENTAL MODAL ANALYSES OF A viola caipira COMPLETE BODY}

This appendix contains the paper "Modal Analysis of a Brazilian Guitar Body", published in the proceeding of the International Symposium on Musical Acoustics (ISMA 2014), held in Le Mans (France). It is presented a finite element model of the complete body of the viola caipira without strings. Natural frequencies and mode shapes are computed by means of a numerical modal analysis and compared with results obtained experimentally. 


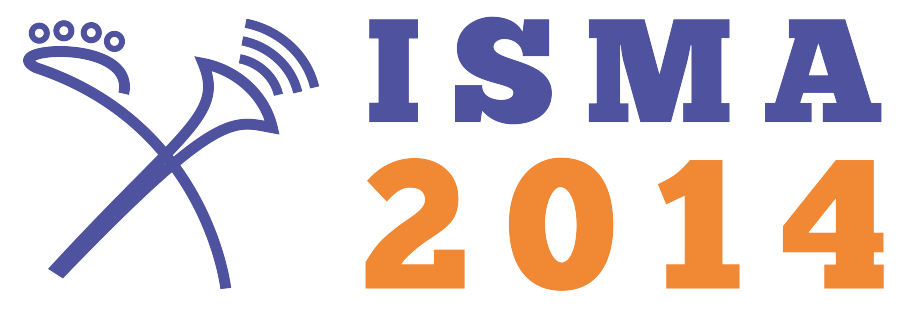

\section{Modal Analysis of a Brazilian Guitar Body}

G. Paiva and J.M.C. Dos Santos

University of Campinas, Rua Mendeleyev, 200, Cidade Universitária ZeferinoVaz, 13083-860 Campinas, Brazil

pitupaiva@ fem.unicamp.br 
The Brazilian guitar is a countryside musical instrument and presents different characteristics that vary regionally by configuring as a sparse group of string musical instruments. Basically, the instrument diversity comes from different geometries of resonance box, shapes of sound hole, types of wood, different tunings, and number and arrangement of strings. This paper intends to present the numerical and experimental modal analysis of a Brazilian guitar, without strings, in a free boundary condition. The modal analysis technique is applied in the determination of the natural frequencies and the corresponding mode shapes. The main dimensions of an actual Brazilian guitar body are used to build the computational model geometry. The numerical modal analysis uses finite element method (FEM) to determine the dynamic behavior of the vibroacoustic system, which is composed by the structural and acoustic systems coupled. The experimental modal analysis is carried out in an actual Brazilian guitar body, where the structural modal parameters (frequency and mode shape) are extracted and used to update the numerical model. Finally, numerical and experimental results are compared and discussed.

\section{Introduction}

The relationship between measurable physical properties of a musical instrument and the subjective evaluation of their sound quality and performance is an important subject of musical acoustics research. Therefore, analytical and numerical methods have been employed to predict and describe accurately the vibroacoustic behavior of complex systems like plucked string instruments [1-4]. With the advent of technology and consequent improvement of the computational processing, numerical models have been used to simulate complex systems and to calculate modal parameters (natural frequency, damping rate and mode shape), which have substantial effect over the tone and sound power desired for a musical instrument [5]. Hence, simulation tools seem to be valuable for the musical instrument design. In general, the luthier, a craftsman who makes or repairs lutes and other string instruments, do not have this facility. In the building process it is required to deal with different types of wood, with well-defined characteristics, which makes impractical this type of investigation.

Experimental tests and numerical simulations by Finite Element Method (FEM) have been previously applied to obtain modal parameters for the classical guitar [1, 2, 6-10]. However, very few have been founded for others types of guitar [2, 4, 11]. In a previous paper [12], the authors presented an application for a numerical modal analysis of a Brazilian guitar resonance box.

This paper presents a numerical and experimental modal analysis of a Brazilian guitar, without strings, in a free boundary condition. The main dimensions of an actual Brazilian guitar body are used to build the computational model geometry. The numerical modal analysis uses ANSYS-FEM to determine the dynamic behavior of the vibroacoustic system, which includes the structural (wood components) and acoustic (fluid inside guitar box + sound hole) systems coupled. The experimental modal analysis is carried out in an actual Brazilian guitar body, where the structural modal parameters are extracted by Polymax method and used to update the numerical model. Finally, numerical and experimental results are compared and discussed.

\section{The Brazilian Guitar}

Par excellence, the Brazilian guitar is a countryside musical instrument. It presents different characteristics that differ regionally, by configuring as a sparse group of string musical instruments. Basically, the instrument diversity comes from different geometries of resonance box, shapes of soundhole, types of wood, different tunings, and number and arrangement of strings. However, this diversity regards to different Brazilian cultural expressions in which this musical instrument is seen as a ritualistic tool. Thus, the expression "Brazilian Guitar" is capable to qualify the instrument in all its variations. According to Corrêa [13], there are six great groups of this instrument: viola caipira (or viola cabocla), viola de cocho, viola de buriti, viola machete, viola nordestina and viola de fandango. This paper is focused on viola caipira, which is the most known and played in all regions of Brazil, particularly in the Southeast and Midwest regions. The viola caipira is derived from the Portuguese guitar, which arrived in Brazil through the Portuguese settlers from different regions and has passed to be used by the Jesuits in the Indian catechesis [14].

Generally, the viola caipira has 10 strings combined at five pairs. Two pairs are tuned in a sharp note on the same fundamental frequency, i.e., the same note at the same height (unison), while the remaining pairs are tuned to the same note, but with a difference of one octave in the height (rate 2:1). The main external parts of a viola caipira are similar to a classic guitar, as shown in Fig. 1a.

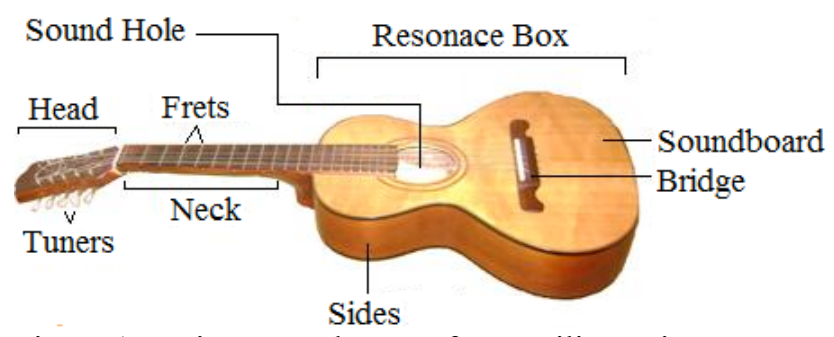

Figure 1: Main external parts of a Brazilian guitar.

The resonance box is composed by top plate (soundboard), back plate, sides and internal structures. These parts enclose the acoustic cavity, which communicates with the external air through the sound hole. The strings are attached to the soundboard through the bridge. Inside the resonance box (Figure 2) there are also fixtures and reinforcements, so as to soundboard harmonic braces; sound hole plates; braces; lining; neck and tail blocks.

Different types of wood are used for the soundboard. In general, luthiers and manufacturers use German Spruce (Picea alpestris), Pau-Marfim (Balfourodendron riedelianum) and Sitka Spruce (Picea sitchensis). There are countless types of wood used for the back plate and sides. Common options include Pau Ferro (Machaerium scleroxylon), Imbuia (Ocotea porosa), Brazilian Mahogany (Swietenia macrophylla), Cedar (Cedrus), Brazilian 
Rosewood (Dalbergia nigra), Indian Rosewood (Dalbergia sissoo), the latter being the most widely used today. Usually, the neck of guitars is made of Cedar, Mahogany or Red Oak (Quercus rubra) and the neck and tail blocks are made of the same wood of soundboard. The scale is generally made of Ebony (Diospyros mespiliformis) or Pau Ferro. The tuners are composed by plastic buttons and tuning keys made of nickel, brass or steel. The frets are made of steel or brass. Usually, there are violas caipira with scales of 10 to 22 frets. It is not possible to establish with accuracy, a standard size for viola. Some have very small size, such as the viola machete (from Bahia). Others are intermediate-sized and large size (from Paraná). Roughly, it is possible to find sizes between 30 and $50 \mathrm{~cm}$ for the length of the largest bulge [13].

\section{Numerical and Experimental Modal Analysis}

The numerical simulations of the Brazilian guitar body were implemented using the ANSYS-FEM software (release 13.0), which offers the possibility to obtain the vibrational behavior of complex mechanical structures. The geometry of the instrument body was designed using the parts and dimensions of a commercial viola caipira, brand Rozini, and model Ponteio Profissional. Figure 2 shows the guitar main dimensions and the components included in the numerical model.

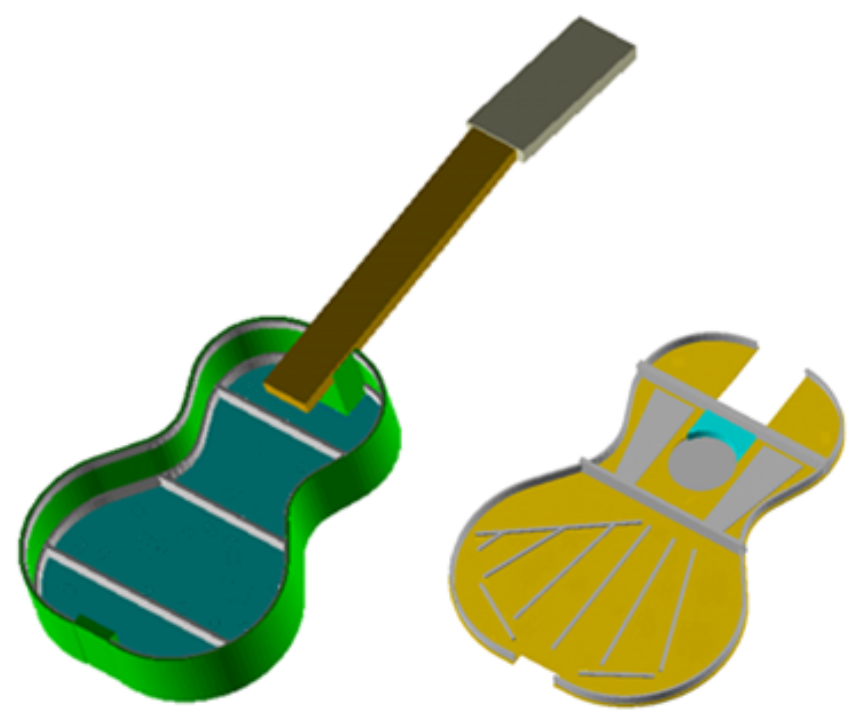

(a) Back Plate and Sides

(b) Soundboard

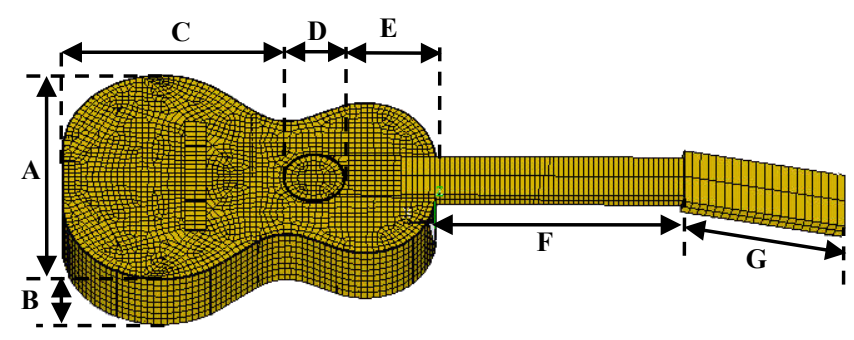

\begin{tabular}{|c|c|c|c|c|c|c|}
\hline A & B & C & D & E & F & G \\
\hline 319 & 83 & 301 & 71 & 98 & 300 & 200 \\
\hline
\end{tabular}

(c) Dimensions [mm].

Figure 2: Internal parts in numerical model: $(a)$ back plate and sides; (b) soundboard; and (c) main dimensions and discretization.
The difficulty to identify some wood types led to assume indications from the literature [16]. Same when it was possible to identify the wood component was not found its mechanical properties, leading to the use of similar timber properties. Thus, it is assumed that the soundboard, tail block, neck block and internal reinforcements are made of Sitka Spruce (Picea sitchensis). The back plate, sides and neck-soundboard junction are made of Yellow Birch (Betula alleghaniensis). Neck and head are made of Red Oak (Quercus rubra). Tuners and frets were not included. Table 1 shows the mechanical properties of woods used in FE model. The thicknesses of soundboard, back plate and sides are $3.0 \mathrm{~mm}, 3.5 \mathrm{~mm}$ and $2.0 \mathrm{~mm}$, respectively.

Table 1 - Wood mechanical properties.

\begin{tabular}{|c|ccc|}
\cline { 2 - 4 } \multicolumn{1}{c|}{} & Sitka Spruce & Yellow Birch & Red Oak \\
\hline $\mathrm{E}_{\mathrm{x}}[\mathrm{MPa}]$ & 10,340 & 11,320 & 14,110 \\
$\mathrm{E}_{\mathrm{y}}[\mathrm{MPa}]$ & 800 & 880 & 2,219 \\
$\mathrm{E}_{\mathrm{z}}[\mathrm{MPa}]$ & 440 & 560 & 1,181 \\
$\mathrm{G}_{\mathrm{xz}}[\mathrm{MPa}]$ & 660 & 830 & 1,282 \\
$\mathrm{G}_{\mathrm{xy}}[\mathrm{MPa}]$ & 630 & 760 & 1,167 \\
$\mathrm{G}_{\mathrm{yz}}[\mathrm{MPa}]$ & 30 & 190 & - \\
$v_{\mathrm{xz}}$ & 0.372 & 0.426 & 0.350 \\
$v_{\mathrm{xy}}$ & 0.467 & 0.451 & 0.448 \\
$v_{\mathrm{zy}}$ & 0.435 & 0.697 & 0.560 \\
\hline$\rho\left[\mathrm{Kg} / \mathrm{m}^{3}\right]$ & 460 & 668 & 700 \\
\hline
\end{tabular}

Vibroacoustic numerical model considers the body structure without strings with the cavity filled with air (internal acoustic model). The ANSYS structure is modelled with SHELL63 element for plates, BEAM188 element for beams and SOLID65 element for solids as tail and neck blocks. The air inside resonance box is modelled with FLUID30 element. Except for the beam element all other elements use orthotropic material model. Fluidstructure interaction is obtained with the coupling matrices, which lead to an asymmetric matrix eigenproblem solution.

A mapped mesh (Figure 2c) is constructed in solid and fluid geometries, which contains around 22,600 elements and 19,000 nodes. Mesh discretization allows response up to $1000 \mathrm{~Hz}$. [17]. A vibroacoustic modal analysis is performed with structural free boundary condition and null pressure in the sound hole.

Table 2 shows the first 5 numeric natural frequencies and mode shapes for the soundboard. Qualitatively the results are consistent with that founded in literature for classic guitars, and also demonstrated by the authors in previous paper [19].

Experimental test used in this work is the Experimental Modal Analysis (EMA) technique [18]. The Frequency Response Functions (FRFs) are obtained through SISO (single-input-single-output) measurement techniques in a free boundary condition at the normal direction to the soundboard. Force excitation is provided by a small electrodynamics shaker, through the stinger and the force transducer, located on the bridge (Figure 3). The force transducer was attached to the bridge with superglue. 
Table 2 - First 5 numerical vibroacoustic modes of the Brazilian guitar body.

\begin{tabular}{|c|c|c|}
\hline $\begin{array}{c}\boldsymbol{f} \\
{[\mathrm{Hz}]}\end{array}$ & $\begin{array}{l}\text { ACOUSTIC } \\
\text { PRESSURE }\end{array}$ & $\begin{array}{c}\text { STRUCTURAL } \\
\text { DISPLACEMENT }\end{array}$ \\
\hline 78 & & \\
\hline 143 & & \\
\hline 151 & & \\
\hline 262 & & \\
\hline 278 & & \\
\hline
\end{tabular}

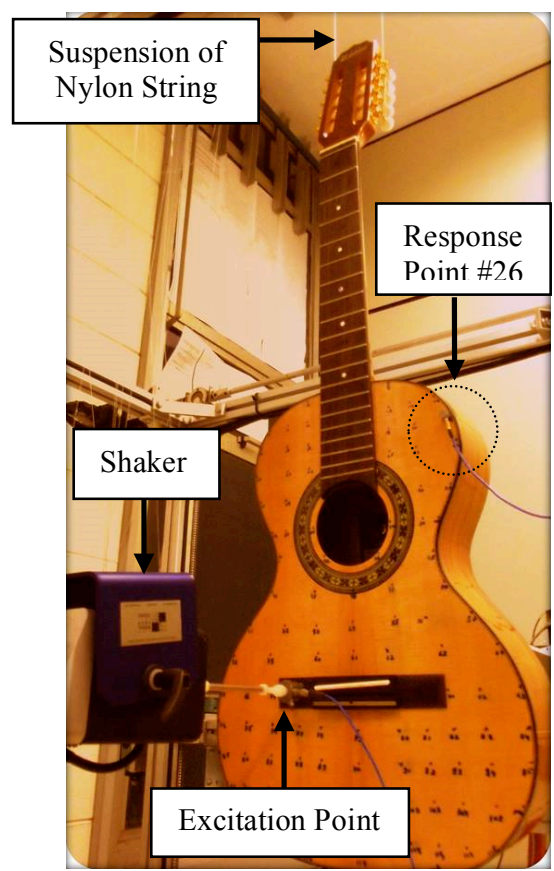

Figure 3: Experimental setup used in the modal analysis of the Brazilian guitar body without strings.

Excitation point at the bridge was choosing to avoid nodal regions, at least for the first five modes. Response measurement points were distributed as following: 111 points on the soundboard, 118 points on the back plate and
10 points on the neck and head. The experimental point coordinates are coincident as the FE model coordinates.

Both excitation and response measurements are in the normal direction to the plates. The excitation signal is a periodic chirp in a frequency range of DC-20kHz. The response is obtained from an accelerometer, which is attached to the successive points by a thin wax layer. Table 4 presents the measurement equipment used.

Modal analysis was conducted at a frequency range from DC-1024 Hz with a $2.0 \mathrm{~Hz}$ resolution was used. For each measurement point, 50 averages were automatically calculated with $75 \%$ overlapping. The FRFs measurements were performed using LMS TestLab, and the modal parameters were obtained by LMS Polymax.

Table 4 - Measurement Equipment

\begin{tabular}{|l|l|l|l|}
\hline Device & Model & Range & Sensitivity \\
\hline Accelerometer & PCB & $0.5 \mathrm{~Hz}-$ & 0.15 \\
& $352 \mathrm{C65}$ & $10 \mathrm{kHz}$ & {$\left[\mathrm{mV} / \mathrm{m} / \mathrm{s}^{2}\right]$} \\
\hline Force sensor & PCB & $0.7 \mathrm{~Hz}-$ & 9.97 \\
& $288 \mathrm{D} 01$ & $7 \mathrm{kHz}$ & {$[\mathrm{mV} / \mathrm{N}]$} \\
\hline Shaker & TMS & up to $31 \mathrm{~N}$ & ---------- \\
& K2004E01 & & \\
\hline FFT Analyzer & LMS & ---------- & ---------- \\
& Scadas & & \\
& SCR05 & & \\
\hline
\end{tabular}

\section{$4 \quad$ Results and Discussions}

In order to compare the experimental results with the numerical ones, the computational model was updated varying the mechanical properties of woods (elastic modules and density). Table 4 presents the comparison of the firsts 8 structural natural frequencies and mode shapes obtained with the numerical vibroacoustic updated model and the experimental measurements on Brazilian guitar body.

Modes 2 and 4 were not obtained in the experimental results. This happens because these modes are predominantly lateral displacements, which could not be detected by the uniaxial accelerometer used to measure only displacements normal to the soundboard.

Soundboard numerical modes 1, 3, 5 and 7 and back plate numerical modes $1,3,6$ and 7 are in good agreement with their corresponding experimental modes. However, soundboard numerical mode 6 and back plate numerical mode 5 seem to be in opposition of phase to their experimental modes.

Note that experimental modes $7(\mathrm{f}=317 \mathrm{~Hz})$ and $8(\mathrm{f}=$ $327 \mathrm{~Hz}$ ) are not two modes as obtained with the shaker excitation (Figure 4). Tests performed with a hammer excitation shows that only one natural frequency actually should be identified in this region. The bifurcation in two different natural frequencies probably occurs because of added mass and inertia moment generated by the force sensor at the shaker excitation setup. In this frequency region the numerical model, which does not include the excitation setup, only presents the mode $7(\mathrm{f}=287 \mathrm{~Hz})$, as shown in Table 4. 
Table 4 - First 8 natural frequencies and mode shapes obtained with the numerical updated model and the experimental measurements.

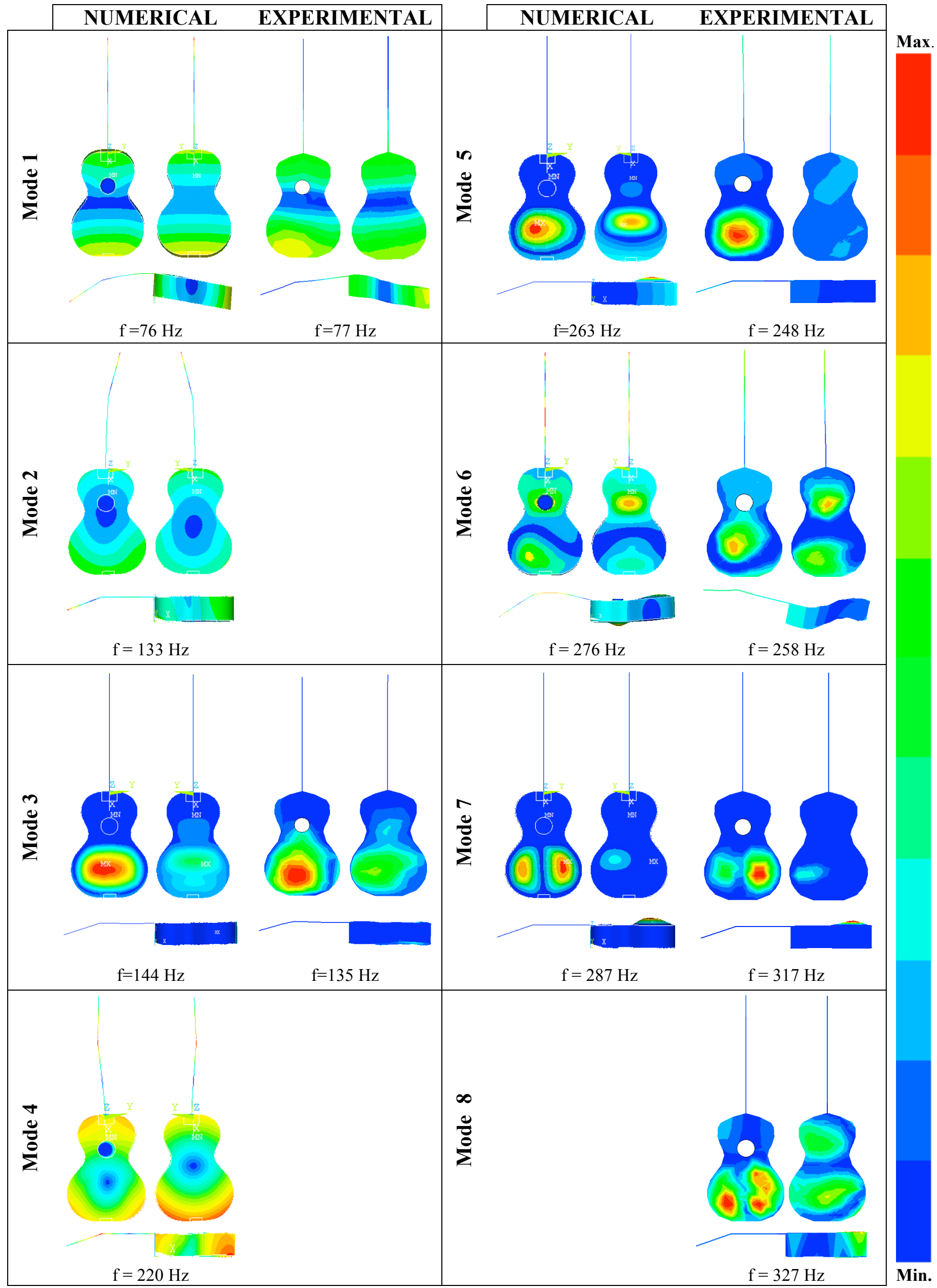




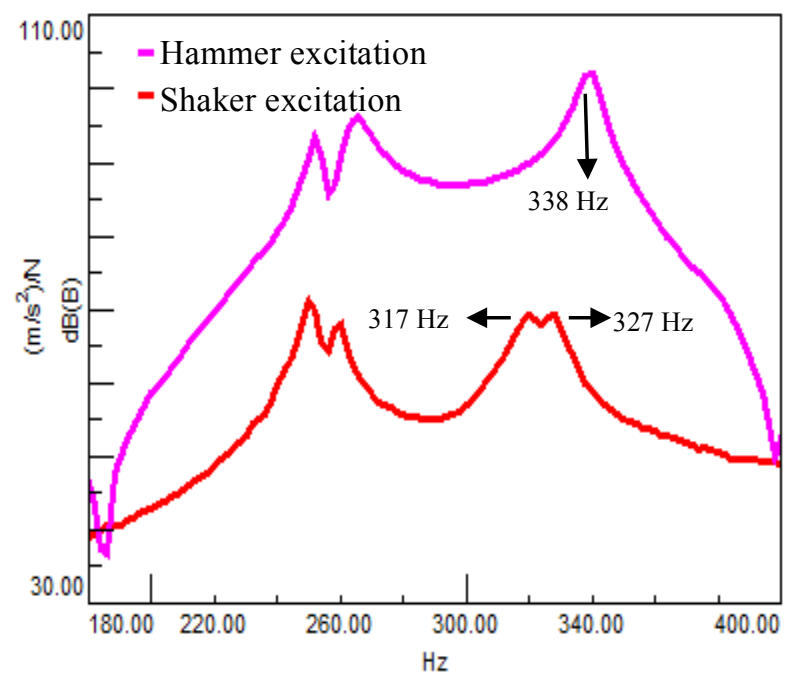

Figure 4: FRF curves (accelerance) obtained through hammer excitation $(-)$ and shaker excitation $(-)$ for the same excitation and response points.

The identified modes 1 and 6 behave as guitar body global modes, which are similar to the flexural beam modes. Mode 1 looks like the first bending mode of a freefree beam and mode 6 as the second bending mode. Identified modes 3, 5, and 7 behave as guitar body local modes, which are plate modes. In general, the largest amplitudes are situated in the lower and middle zones of the soundboard and back plate for the analyzed frequency band. Mode 3 is the fundamental mode of the resonance box, where the soundboard presents an antinodes zone at the position of the bridge. The upper zone of the soundboard remains motionless due to the presence of the soundboard braces and neck block. In the mode 7 the back remains motionless: the internal reinforcements difficult the vibration of its transversal flexural patterns and thus the contribution to radiation due to the back will be negligible.

The results for the natural frequencies present errors ranging from below $7 \%$ (modes $1,3,5,6$ ) to almost $10 \%$ (mode 7 ), being the highest error the mode 7 (9.4\%) and the lowest with mode $1(1.0 \%)$.

The disagreement between some results of natural frequencies and mode shapes can also be attributed to the simplifications assumed in computer simulation. Also, the lacquer layer and the truss rod and tuners were not considered in the computational model. The applied shaker excitation, force sensor and accelerometer modify significantly the experimental modal identification in relation to the real structure since they add mass and inertia moment $o$ the guitar body in the region of the measurement point.

\section{Conclusion}

The Brazilian guitar was briefly described with regard to its historical, structural and acoustical characteristics. A procedure for vibroacoustic modal analysis by finite element method with ANSYS software was developed. The computational results were verified with experimental data obtained from an experimental modal analysis. In general, it was observed that the finite element method proved to be effective and can determine the dynamic behavior of the Brazilian guitar body. However, in order to get good results from the computational model and to minimize de model updating process, it is very important to identify and characterize the mechanical properties of all woods used. The influence of the excitation and measurements process is very important due to the guitar low weight and acoustic sensitivity. Future works are under way using impact hammer excitation and laser vibrometer measurements. Furthermore, it must be observed that the determination of the modal characteristics of the guitar body is only an initial stage about the study to obtain tones and sound power desired for stringed instruments. But, this information is a helpful tool for craftsmen and manufactures to get more control over the quality of the instrument in different stages of its construction. Finally, to go further in this research we need additional tests and psychoacoustic measurements of the sound field, which explain subjective evaluations and allow their relationship with objective parameters.

\section{Acknowledgments}

The authors would like to thank Rozini Instrumentos Musicais, CNPq, Fapesp, and CAPES for supporting this work.

\section{References}

[1] H. Wright, "The acoustics and psychoacoustics of the guitar", University of Wales, College of Cardiff, $\mathrm{PhD}$ Thesis, 260p (1996)

[2] N. H. Fletcher, T. D. Rossing, "The Physics of Musical Instruments", Springer, New York, 2nd ed. (2005)

[3] B. Elie, F. Gautier, B. David, "Macro parameters describing the mechanical behavior of classical guitars", $J$. Acous. Soc. Am. 132(6), 4013-4024 (2012)

[4] V. Debut, M. Carvalho, M. Marques, J. Antunes, "Three-dimensional time-domain simulations of the string/soundboard coupled dynamics for a twelve-string Portuguese guitar", Proceedings of $12^{\text {th }}$ French Acoustics Congress, France (2014)

[5] M. Brooke, "Modelling guitar radiation fields using the boundary element method", University of Wales, College of Cardiff, PhD Thesis, 197p (1992)

[6] R. M. French, "Engineering the Guitar", Springer, New York, 266 p. (2009)

[7] I. Curtu, M. D. Stanciu, N. C. Cretu, C. I. Rosca, "Modal Analysis of different types of classical guitar bodies", Proceedings of the $10^{\text {th }}$ WSEAS International Conference on Acoustics \& Music: Theory \& Applications, $6 \mathrm{p}(2009)$

[8] M. J. Elejabarrieta, A. Ezcurra, C. Santamaría, "Coupled modes of resonance box of the guitar", J. Acoust. Soc. Am. 111(5), 2283-2292 (2002)

[9] M. J. Elejabarrieta, A. Ezcurra, C. Santamaría, “Air Cavity Modes in the Resonance Box of the Guitar: The Effect of Sound Hole", Journal of Sound and Vibration 252(3), 584-590 (2002)

[10] J. Bretos, C. Santamaria, J. Alonso-Moral, "Vibrational patterns of a violin shaped air cavity obtained finite element modeling", Acustica (85), 1- 3 (1999) 
[11] O. Inácio, F. Santiago, P. C. Cabral, "The Portuguese Guitar Acoustics Part 1 - Vibroacoustic Measurements", Acústica, Portugal (2004)

[12] G. O. Paiva, J. M. C. Dos Santos, "Vibroacoustic Modal Analysis of a Brazilian Guitar Resonance Box", International Proceedings of the ICEDyn Conference on Structural Engineering Dynamics, Portugal (2013)

[13] R. Corrêa, "A arte de pontear viola", $2^{a}$ ed., Brasília, Viola Corrêa, 259p. (2002)

[14] I. Vilela, "O caipira e a viola brasileira", Sonoridades luso-afro-brasileiras, $1^{\text {a }}$ ed., Lisboa: Imprensa de Ciências Sociais do Instituto de Ciências Sociais da Universidade de Lisboa, pp. 171-187 (2004)

[15] O. C. Zienkiewicz, E. Newton, "Coupled Vibrations of a Compressible Fluid", Proceedings of International Symposium of Finite Elements Techniques, Stuttgart, pp. 359-379 (1969)

[16] R. Bergman et al, "Wood Handbook-Wood as an Engineering Material", General Technical Report FPLGTR-190, Madison, WI: U.S. Department of Agriculture, Forest Service, Forest Products Laboratory, 508 p. (2010)

[17] ANSYS, "ANSYS Analysis Guide for Release 13.0", (2010)

[18] D. J. Ewins, "Modal Testing: Theory and Practice", Research Studies Press. Ltd, (1984)

[19] G. O. Paiva, J. M. C. Dos Santos, “Análise Modal Vibroacústica da Caixa de Ressonância de uma Viola Caipira ", Proceedings of $7^{\text {th }}$ CONEM National Congress of Mechanical Engineer, Brasil (2012) 


\section{INTRODUCTION}

The Brazilian guitar is a countryside musical instrument and presents different characteristics that vary regionally. This work is focused on the viola caipira type, which is derived from the Portuguese guitar and is the most known and played in all regions of Brazil. Generally, the viola caipira (figure 1) has 10 strings combined at five pairs and its main parts are similar to a classic guitar.

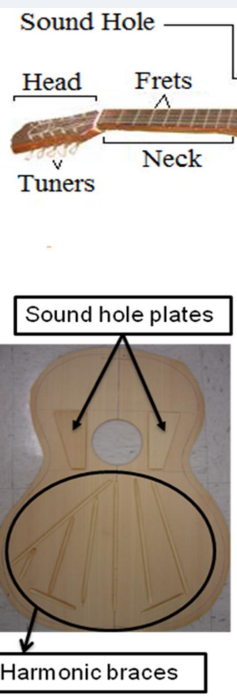

(b)
Resonace Box
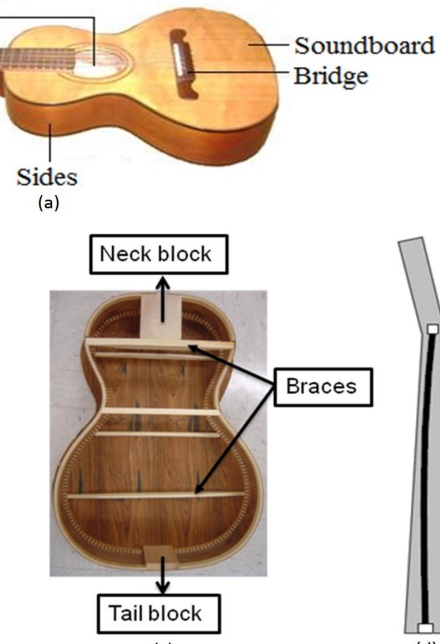

(c)

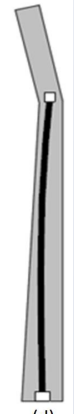

(d)
Figure 1: Main parts of a Brazilian guitar: (a) external body; (b) internal soundboard components; (c) internal back plate and side components; (d) placement of truss rod in the neck.

This work intends to present the numerical and experimental modal analysis applied in the determination of the natural frequencies and the corresponding mode shape of a Brazilian guitar, without strings, in a free boundary condition.

\section{NUMERICAL MODEL}

The Finite Element Method (FEM) is applied. It is assumed that the soundboard, tail block, neck block and internal reinforcements are made of Sitka Spruce (Picea sitchensis). The back plate, sides and neck-soundboard junction are made of Yellow Birch (Betula alleghaniensis). Neck and head are made of Red Oak (Quercus rubra). The ANSYS-FEM structure is modelled with SHELL63 element for plates, BEAM188 element for beams and SOLID65 element for solids as tail and neck blocks. The air inside resonance box is modelled with FLUID30 element. Except for the beam element all other elements use orthotropic material model.

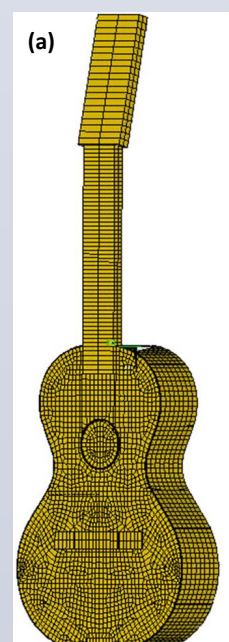

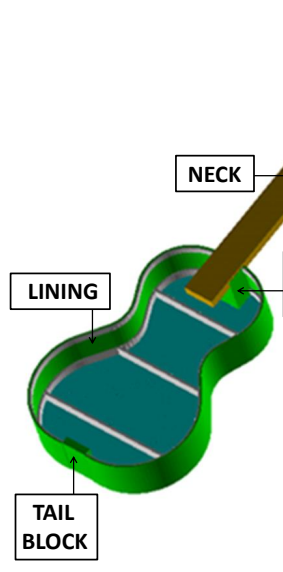

(b)
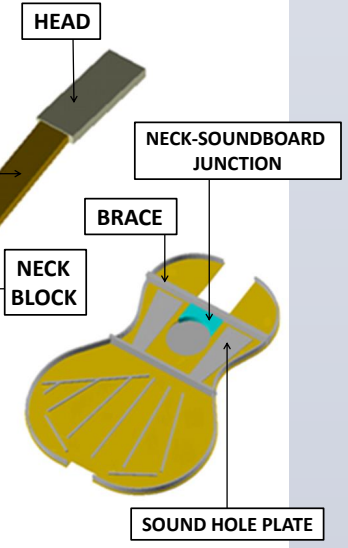

(c)

\section{RESULTS AND DISCUSSIONS}

In order to compare the experimental results with the numerical ones (table 1), the computational model was updated varying the mechanical properties of woods (elastic modules and density).

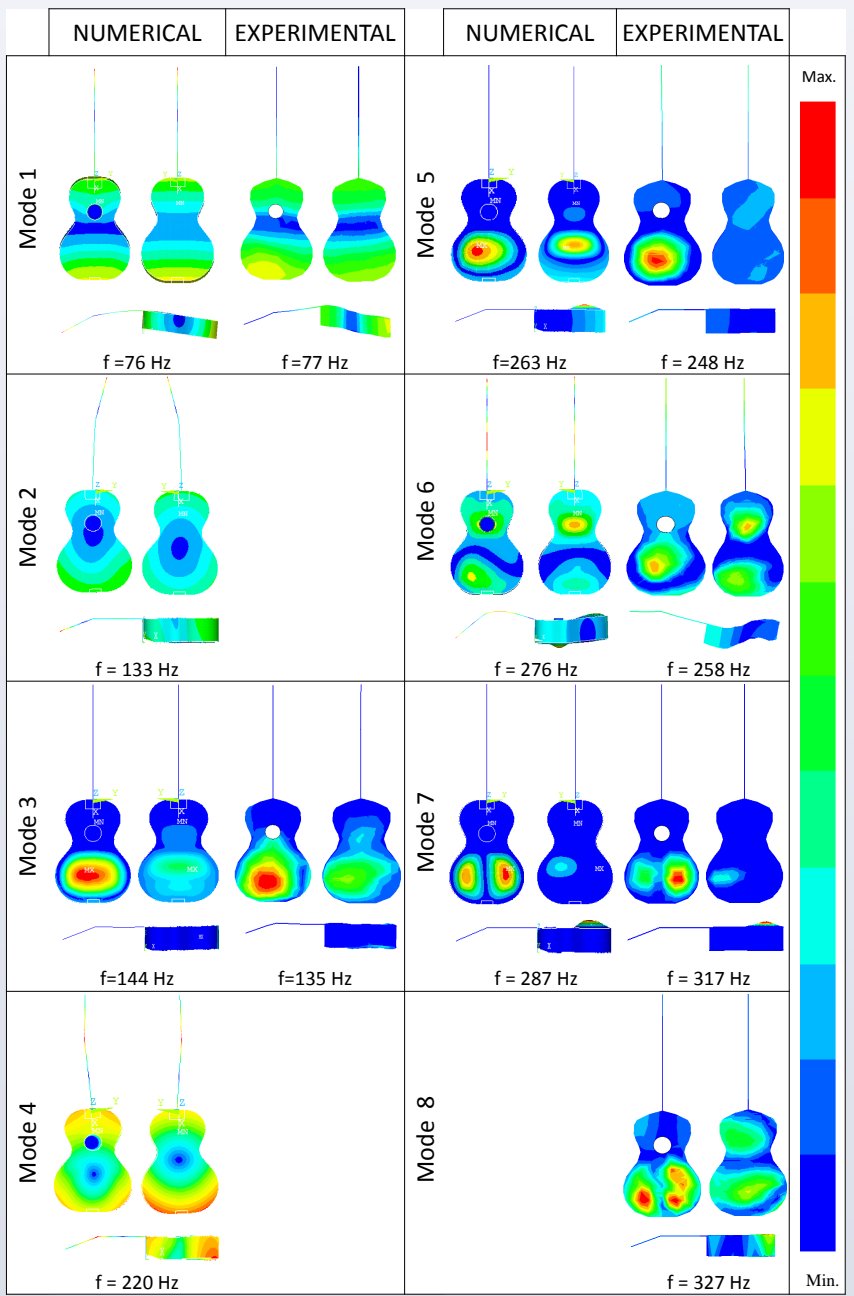

Table 1 - First 8 natural frequencies and mode shapes obtained with the numerical updated model and the experimental measure
independently normalized.

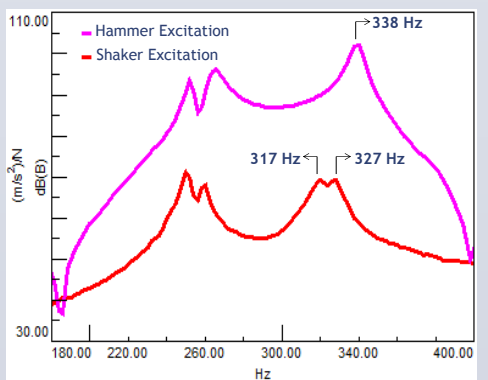

Note that experimental modes 7 ( $f=317$ $\mathrm{Hz})$ and $8(\mathrm{f}=327 \mathrm{~Hz})$ are not two modes as obtained with the shaker excitation (figure 3). Tests performed with a hammer excitation shows that only one natural frequency actually should be identified in this region. The bifurcation in two different natural frequencies probably occurs because of added mass and inertia Figure 3: FRF curves (accelerance) obtained through excitation and response points.

moment generated by the force sensor at the shaker excitation setup.

\section{CONCLUSIONS}

In general, it was observed that the FEM proved to be effective and can determine the dynamic behavior of the Brazilian guitar body. However, in order to get good results from the computational model and to minimize de model updating process, it is very important to identify and characterize the mechanical properties of all woods used. The influence of the excitation and measurements process is very important due to the guitar low weight and acoustic sensitivity. 



\section{BIBLIOGRAPHY}

[1] J.-M. Adrien. The missing link: Modal synthesis. In Representations of musical signals, pages 269-298. MIT Press, 1991.

[2] J.-M. Adrien and X. Rodet. Physical models of instruments: A modular approach, application to strings. In ICMC, 1985.

[3] A. M. Araújo. Viola. Folclore Nacional, p 433-451. Vol. 2 Sao Paulo. 1964.

[4] L. H. C. d. Azevedo. A moda de viola. Rio de Janeiro: Cultura Política, 1943.

[5] R. Bacon and J. Bowsher. A discrete model of a struck string. Acta Acustica united with Acustica, 41(1):21-27, 1978.

[6] R. Badeau, B. David, and G. Richard. A new perturbation analysis for signal enumeration in rotational invariance techniques. IEEE Transactions on Signal Processing, 54(2):450-458, 2006.

[7] B. Bank and M. Karjalainen. Passive admittance matrix modeling for guitar synthesis. In Proc. Conf. on Digital Audio Effects, pages 3-8, 2010.

[8] A. Benade and W. Messenger. Sitar spectrum properties. The Journal of the Acoustical Society of America, 71(S1):S83-S83, 1982.

[9] A. H. Benade. Fundamentals of musical acoustics. Courier Corporation, 1990.

[10] S. Bilbao. Conservative numerical methods for nonlinear strings. The Journal of the Acoustical Society of America, 118(5):3316-3327, 2005.

[11] S. Bilbao. Numerical sound synthesis: finite difference schemes and simulation in musical acoustics. John Wiley \& Sons, 2009.

[12] S. Bilbao. Time domain simulation and sound synthesis for the snare drum. The Journal of the Acoustical Society of America, 131(1):914-925, 2012.

[13] S. Bilbao and A. Torin. Numerical modeling and sound synthesis for articulated string/fretboard interactions. Journal of the Audio Engineering Society, 63(5):336-347, 2015.

[14] S. Bilbao, A. Torin, and V. Chatziioannou. Numerical modeling of collisions in musical instruments. Acta Acustica united with Acustica, 101(1):155-173, 2015.

[15] G. Bissinger. Structural acoustics of good and bad violins. The Journal of the Acoustical Society of America, 124(3):1764-1773, 2008.

[16] R. R. Boullosa, F. Orduña-Bustamante, and A. P. Lopez. Tuning characteristics, radiation efficiency and subjective quality of a set of classical guitars. Applied Acoustics, 56(3):183-197, 1999.

[17] X. Boutillon. Model for piano hammers: Experimental determination and digital simulation. The Journal of the Acoustical Society of America, 83(2):746-754, 1988.

[18] R. M. Brach. Mechanical impact dynamics: rigid body collisions. Brach Engineering, LLC, 2007. 
[19] J. Bridges and M. Van Walstijn. Investigation of tanpura string vibrations using a two-dimensional time-domain model incorporating coupling and bridge friction. Proc. of the third Vienna Talk on Music Acoustics, 2015.

[20] G. Caldersmith. Guitar as a reflex enclosure. The Journal of the Acoustical Society of America, 63(5):1566-1575, 1978.

[21] A. Candido. Os parceiros do Rio Bonito: estudo sobre o caipira paulista ea transformação dos seus meios de vida. Livraria Duas Cidades São Paulo, 1975.

[22] B. Capleton. False beats in coupled piano string unisons. The Journal of the Acoustical Society of America, 115(2):885-892, 2004.

[23] J.-L. L. Carrou, F. Gautier, N. Dauchez, and J. Gilbert. Modelling of sympathetic string vibrations. Acta Acustica united with Acustica, 91(2):277-288, 2005.

[24] J. Chabassier and P. Joly. Energy preserving schemes for nonlinear hamiltonian systems of wave equations: Application to the vibrating piano string. Computer Methods in Applied Mechanics and Engineering, 199(45):2779-2795, 2010.

[25] A. Chaigne. On the use of finite differences for musical synthesis. application to plucked stringed instruments. J. Acoustique, 5(2):181-211, 1992.

[26] A. Chaigne and A. Askenfelt. Numerical simulations of piano strings. i. a physical model for a struck string using finite difference methods. The Journal of the Acoustical Society of America, 95(2):1112-1118, 1994.

[27] A. Chaigne and A. Askenfelt. Numerical simulations of piano strings. ii. comparisons with measurements and systematic exploration of some hammer-string parameters. The Journal of the Acoustical Society of America, 95(3):1631-1640, 1994.

[28] A. Chaigne, A. Askenfelt, and E. Jansson. Temporal synthesis of string instrument tones. Quarterly Progress and Status Report, 4(81-100):167, 1990.

[29] A. Chaigne and V. Doutaut. Numerical simulations of xylophones. i. time-domain modeling of the vibrating bars. The Journal of the Acoustical Society of America, 101(1):539-557, 1997.

[30] O. Christensen. Quantitative models for low frequency guitar function. J. Guitar Acoustics, 6(2):10-25, 1982.

[31] O. Christensen. An oscillator model for analysis of guitar sound pressure response. Acta Acustica united with Acustica, 54(5):289-295, 1984.

[32] O. Christensen and B. B. Vistisen. Simple model for low-frequency guitar function. The Journal of the Acoustical Society of America, 68(3):758-766, 1980.

[33] R. Corrêa. A arte de pontear viola, Brasília, DF. Viola Corrêa, 2000.

[34] R. N. Corrêa. Viola caipira: das práticas populares à escritura da arte. $\mathrm{PhD}$ thesis, Universidade de São Paulo.

[35] R. R. Craig. Structural dynamics: an introduction to computer methods. John Wiley \& Sons Inc, 1981.

[36] F. Crossley. Coefficient of restitution interpreted as damping in vibroimpact. Journal of Applied Mechanics, Transactions ASME, 42, 1975.

[37] C. Cuesta. Corde vibrante isolée mécaniquement, amortissements, non-linéarités; application au clavecin et à la tampoura. PhD thesis, Université du Maine, 1990. 
[38] L. da Câmara Cascudo. Dicionário do folclore brasileiro. Ministério da Educação e Cultura, Instituto Nacional do Livro, 1954.

[39] S. Daltrop, A. Kotlicki, C. Waltham, N. Wolfe, B. Elie, and F. Gautier. Vibroacoustic characteristics of a gothic harp. The Journal of the Acoustical Society of America, 131(1):837-843, 2012.

[40] B. David. Caractérisations acoustiques de structures vibrantes par mise en atmosphère raréfiée. Méthodes d'estimation relatives aux fréquences et amortissements des modes propres. Applications en acoustique musicale. PhD thesis, Université Pierre et Marie Curie-Paris VI, 1999.

[41] M. De Andrade. Dicionário musical brasileiro. Number 162. Ministério da Cultura, 1989.

[42] V. Debut, J. Antunes, M. Marques, and M. Carvalho. Physics-based modeling techniques of a twelve-string portuguese guitar: A non-linear time-domain computational approach for the multiple-strings/bridge/soundboard coupled dynamics. Applied Acoustics, 108:3-18, 2016.

[43] T. A. Deiters, D. L. Hunt, and E. Osborne. A comparison of excitation methods to the taurus vehicle modal test. In Proceedings of the 12th International Modal Analysis, volume 2251, page 26, 1994.

[44] M. Demoucron. On the control of virtual violins-Physical modelling and control of bowed string instruments. PhD thesis, Université Pierre et Marie Curie-Paris VI; Royal Institute of Technology, Stockholm, 2008.

[45] C. Desvages and S. Bilbao. Physical modeling of nonlinear player-string interactions in bowed string sound synthesis using finite difference methods. In Proceedings of the International Symposium on Musical Acoustics, volume 430, 2014.

[46] C. Desvages and S. Bilbao. Two-polarisation finite difference model of bowed strings with nonlinear contact and friction forces. In Int. Conference on Digital Audio Effects (DAFx-15), 2015 .

[47] C. Desvages and S. Bilbao. Two-polarisation physical model of bowed strings with nonlinear contact and friction forces, and application to gesture-based sound synthesis. Applied Sciences, $6(5): 135,2016$

[48] S. S. A. Dias. O processo de escolarização da viola caipira: novos violeiros inventano modas $e$ identidades. PhD thesis, University of São Paulo, 2012.

[49] B. M. Douglas. Quick release pullback testing and analytical seismic analysis of a six span composite girder bridge. Technical report, 1976.

[50] M. Ducceschi and C. Touzé. Modal approach for nonlinear vibrations of damped impacted plates: Application to sound synthesis of gongs and cymbals. Journal of Sound and Vibration, 344:313$331,2015$.

[51] F. Durup and E. V. Jansson. The quest of the violin bridge-hill. Acta Acustica United with Acustica, 91(2):206-213, 2005.

[52] K. Ege, X. Boutillon, and B. David. High-resolution modal analysis. Journal of Sound and Vibration, 325(4):852-869, 2009.

[53] M. Elejabarrieta, A. Ezcurra, and C. Santamarı. Coupled modes of the resonance box of the guitar. The Journal of the Acoustical Society of America, 111(5):2283-2292, 2002.

[54] B. Elie. Vibroacoustic characterization of stringed musical instruments. Application to the lutherie assistance. PhD thesis, Ph. D. thesis, Université du Maine, France, 2012.

[55] B. Elie, F. Gautier, and B. David. Macro parameters describing the mechanical behavior of classical guitars. The Journal of the Acoustical Society of America, 132(6):4013-4024, 2012. 
[56] B. Elie, F. Gautier, and B. David. Estimation of mechanical properties of panels based on modal density and mean mobility measurements. Mechanical systems and signal processing, 40(2):628644, 2013.

[57] B. Elie, F. Gautier, and B. David. Acoustic signature of violins based on bridge transfer mobility measurements. The Journal of the Acoustical Society of America, 136(3):1385-1393, 2014.

[58] G. Evangelista. Physical model of the string-fret interaction. In DAFx'11, Paris, 2011, pages 345-351, 2011.

[59] G. Evangelista and F. Eckerholm. Player-instrument interaction models for digital waveguide synthesis of guitar: Touch and collisions. IEEE transactions on audio, speech, and language processing, 18(4):822-832, 2010.

[60] A. Ezcurra, M. Elejabarrieta, and C. Santamaria. Fluid-structure coupling in the guitar box: numerical and experimental comparative study. Applied Acoustics, 66(4):411-425, 2005.

[61] I. M. Firth. Physics of the guitar at the helmholtz and first top-plate resonances. The Journal of the Acoustical Society of America, 61(2):588-593, 1977.

[62] H. Fletcher. Normal vibration frequencies of a stiff piano string. The Journal of the Acoustical Society of America, 36(1):203-209, 1964.

[63] N. H. Fletcher and T. Rossing. The physics of musical instruments. Springer Science \& Business Media, 2012.

[64] R. M. French. Engineering the guitar: theory and practice. Springer Science \& Business Media, 2008.

[65] V. Fréour, F. Gautier, B. David, and M. Curtit. Extraction and analysis of body-induced partials of guitar tones. The Journal of the Acoustical Society of America, 138(6):3930-3940, 2015.

[66] F. Gautier and N. Dauchez. Acoustic intensity measurement of the sound field radiated by a concert harp. Applied acoustics, 65(12):1221-1231, 2004.

[67] F. Gautier, V. Doutaut, and J. Fouilleul. Lutherie tools: projet collaboratif entre ateliers de lutherie et laboratoires. Musique $\mathcal{E}$ technique, 4:21-28, 2009.

[68] M. Géradin and D. J. Rixen. Mechanical vibrations: theory and application to structural dynamics. John Wiley \& Sons, 2014.

[69] M. Gharib and Y. Hurmuzlu. A new contact force model for low coefficient of restitution impact. Journal of Applied Mechanics, 79(6):064506, 2012.

[70] N. Giordano and M. Jiang. Physical modeling of the piano. EURASIP Journal on Applied Signal Processing, 2004:926-933, 2004.

[71] C. Gough. The theory of string resonances on musical instruments. Acta Acustica united with Acustica, 49(2):124-141, 1981.

[72] B. Gustafsson, H.-O. Kreiss, and J. Oliger. Time dependent problems and difference methods, volume 24. John Wiley \& Sons, 1995.

[73] I. Guyard and A. Chaigne. Musical sound synthesis by finite differences method: Pcimplementation and new developments. In Audio Engineering Society Convention 84. Audio Engineering Society, 1988.

[74] H. Hertz. Miscellaneous papers. Macmillan, 1896.

[75] T. Hill, B. Richardson, and S. Richardson. Acoustical parameters for the characterisation of the classical guitar. Acta Acustica united with Acustica, 90(2):335-348, 2004. 
[76] L. Hiller and P. Ruiz. Synthesizing musical sounds by solving the wave equation for vibrating objects: Part 1. Journal of the Audio Engineering Society, 19(6):462-470, 1971.

[77] L. Hiller and P. Ruiz. Synthesizing musical sounds by solving the wave equation for vibrating objects: Part 2. Journal of the Audio Engineering Society, 19(7):542-551, 1971.

[78] J. Hoekstra. Properties and applications of enamelled wire. Philips Technical Review, 3(2):40-47, 1938.

[79] O. Inácio, J. Antunes, and M. Wright. Computational modelling of string-body interaction for the violin family and simulation of wolf notes. Journal of Sound and Vibration, 310(1):260-286, 2008 .

[80] C. Issanchou, S. Bilbao, J.-L. Le Carrou, C. Touzé, and O. Doaré. A modal-based approach to the nonlinear vibration of strings against a unilateral obstacle: Simulations and experiments in the pointwise case. Journal of Sound and Vibration, 393:229-251, 2017.

[81] C. Issanchou, J.-L. Le Carrou, C. Touzé, B. Fabre, and O. Doaré. String/frets contacts in the electric bass sound: Simulations and experiments. Applied Acoustics, 129:217-228, 2018.

[82] E. V. Jansson. A study of acoustical and hologram interferometric measurements of the top plate vibrations of a guitar. Acta Acustica united with Acustica, 25(2):95-100, 1971.

[83] E. V. Jansson. Acoustics for the guitar maker. Function, construction and quality of the guitar. Royal Swedish Academy of Music, Publication, (38):27-50, 1983.

[84] H. Järveläinen and M. Karjalainen. Perceptibility of inharmonicity in the acoustic guitar. Acta Acustica united with Acustica, 92(5):842-847, 2006.

[85] H. Järveläinen, V. Välimäki, and M. Karjalainen. Audibility of the timbral effects of inharmonicity in stringed instrument tones. Acoustics Research Letters Online, 2(3):79-84, 2001.

[86] P. Joly, L. Rhaouti, and A. Chaigne. Numerical simulation of timpani. The Journal of the Acoustical Society of America, 105(2):1125-1125, 1999.

[87] M. Karjalainen, V. Välimäki, and T. Tolonen. Plucked-string models: From the karplus-strong algorithm to digital waveguides and beyond. Computer Music Journal, 22(3):17-32, 1998.

[88] D. Kartofelev, A. Stulov, H.-M. Lehtonen, and V. Välimäki. Modeling a vibrating string terminated against a bridge with arbitrary geometry. In Proc. Stockholm Musical Acoust. Conf, 2013.

[89] J. Lai and M. Burgess. Radiation efficiency of acoustic guitars. The Journal of the Acoustical Society of America, 88(3):1222-1227, 1990.

[90] C. Lambourg and A. Chaigne. Measurements and modeling of the admittance matrix at the bridge in guitars. Proceedings of SMAC'93, pages 448-453, 1993.

[91] J. P. Lauffer, T. G. Carne, and T. D. Ashwill. Modal testing in the design evaluation of wind turbines. Sandia National Laboratories, 1988.

[92] J. Le Carrou, F. Gautier, and E. Foltête. Experimental study of a0 and t1 modes of the concert harp. The Journal of the Acoustical Society of America, 121(1):559-567, 2007.

[93] J.-L. Le Carrou, F. Gautier, and R. Badeau. Sympathetic string modes in the concert harp. Acta Acustica united with Acustica, 95(4):744-752, 2009.

[94] J. le Rond D'Alembert. Recherches sur la courbe que forme une corde tendue mise en vibrations. Histoire de l'Académie Royale des Sciences et Belles Lettres (Année 1747), 3:214-249, 1747.

[95] E. Lieber. Moderne theorien über die physik der schwingenden saite und ihre bedeutung für die musikalische akustik. Acustica, 33:324-335, 1975. 
[96] R. T. d. Lima. Estudo sobre a viola. Revista Brasileira de Folclore, Rio de Janeiro, 4(8/10):29-38, 1964.

[97] A. Mandal and P. Wahi. Natural frequencies, modeshapes and modal interactions for strings vibrating against an obstacle: Relevance to sitar and veena. Journal of Sound and Vibration, 338:42-59, 2015.

[98] J. Marecos, M. Castanheta, and J. T. Trigo. Field observation of tagus river suspension bridge. Journal of the Structural Division, 95(4):555-583, 1969.

[99] J. d. S. Martins. A dupla linguagem na cultura caipira. Sonoridades Luso-Afro-Brasileiras, Lisboa, Imprensa de Ciências Sociais, pages 189-226, 2004.

[100] J. Meyer. Quality aspects of the guitar tone. Function, construction, and quality of the guitar. Stockholm: Royal Swedish Academy of Music, 1983.

[101] J. D. Morrison and J.-M. Adrien. Mosaic: A framework for modal synthesis. Computer Music Journal, 17(1):45-56, 1993.

[102] S. Ohlsson. Modal testing of the tjorn bridge. In Proc., 4th Int. Modal Analysis Conf, pages 599-605, 1986.

[103] E. V. Oliveira. Instrumentos musicais populares portugueses. Fundação Calouste Gulbenkian/Museu Nacional de Etnologia, 2000.

[104] S. I. Orr. Numerical simulation of coupled strings with application to physics-based sound synthesis. PhD thesis, Queen's University of Belfast, 2013.

[105] R. Osgood. Dynamic characterization testing of wind turbines. Technical report, National Renewable Energy Lab., Golden, CO (US), 2001.

[106] G. O. Paiva. Análise modal vibroacústica da caixa de ressonância de uma viola caipira. Master's thesis, University of Campinas, 2013.

[107] G. O. Paiva and J. Dos Santos. Análise modal vibroacústica da caixa de ressonância de uma viola caipira. In International Proceedings of the 7th Congress of Mechanical Engineering, Brazil, 2012.

[108] G. O. Paiva and J. Dos Santos. Vibroacoustic modal analysis of a brazilian guitar resonance box. In International Proceedings of the ICEDyn Conference on Structural Engineering Dynamics, Portugal, 2013.

[109] A. Paté, J.-L. Le Carrou, and B. Fabre. Predicting the decay time of solid body electric guitar tones. The Journal of the Acoustical Society of America, 135(5):3045-3055, 2014.

[110] R. C. Pedro. Uma orquestra de viola caipira do município de são carlos. Master's thesis, University of Campinas, 2013.

[111] B. Peeters, G. Lowet, H. Van der Auweraer, and J. Leuridan. A new procedure for modal parameter estimation. Sound and Vibration, 38(1):24-29, 2004.

[112] J. P. d. A. Pinto. A viola caipira de Tião Carreiro. PhD thesis, University of Campinas, 2008.

[113] J. E. Popp. Four mass coupled oscillator guitar model. The Journal of the Acoustical Society of America, 131(1):829-836, 2012.

[114] M. R. Portnoff. A quasi-one-dimensional digital simulation for the time-varying vocal tract. $\mathrm{PhD}$ thesis, Massachusetts Institute of Technology, 1973.

[115] C. V. Raman. On the mechanical theory of the vibrations of bowed strings and of musical instruments of the violin family, with experimental verification of the results. Indian Assoc. Cultivation Sci. Bull, 15:1-158, 1918. 
[116] C. V. Raman. On some indian stringed instruments. Proc. Indian Assoc. Cultiv. Sci, 7:29-33, 1921.

[117] E. Rank and G. Kubin. A waveguide model for slapbass synthesis. In Acoustics, Speech, and Signal Processing, 1997. ICASSP-97., 1997 IEEE International Conference on, volume 1, pages 443-446. IEEE, 1997.

[118] R. A. Rasch and V. Heetvelt. String inharmonicity and piano tuning. Music Perception: An Interdisciplinary Journal, 3(2):171-189, 1985.

[119] L. Rhaouti, A. Chaigne, and P. Joly. Time-domain modeling and numerical simulation of a kettledrum. The Journal of the Acoustical Society of America, 105(6):3545-3562, 1999.

[120] T. D. Rossing, J. Popp, and D. Polstein. Acoustical response of guitars. In Proc. of SMAC, volume 83,1985

[121] P. M. Ruiz. A technique for simulating the vibration of strings with a digital computer. Master's thesis, University of Illinois at Urbana-Champaign, 1970.

[122] J. A. Sardinha and L. Guerra. Viola campaniça: o outro Alentejo. 2001.

[123] J. C. Schelleng. Erratum: The violin as a circuit [j. acoust. soc. am. 35, 326-338 (1963)]. The Journal of the Acoustical Society of America, 35(8):1291-1291, 1963.

[124] M. R. Schroeder. Response to "comments on "new method of measuring reverberation time"'[pw smith, jr., j. acoust. soc. am. 38, 359 (1)(1965)]. The Journal of the Acoustical Society of America, 38(2):359-361, 1965.

[125] O. Schuck and R. Young. Observations on the vibrations of piano strings. The Journal of the Acoustical Society of America, 15(1):1-11, 1943.

[126] S. Siddiq. A physical model of the nonlinear sitar string. Archives of acoustics, 37(1):73-79, 2012.

[127] E. Skudrzyk. The mean-value method of predicting the dynamic response of complex vibrators. The Journal of the Acoustical Society of America, 67(4):1105-1135, 1980.

[128] J. O. Smith III. Efficient synthesis of stringed musical instruments. 1993.

[129] D. Speiser and K. Williams. Discovering the Principles of Mechanics 1600-1800: Essays by David Speiser, volume 1. Springer Science \& Business Media, 2008.

[130] G. G. Stokes. On the effect of the internal friction of fluids on the motion of pendulums, volume 9. Pitt Press Cambridge, 1851.

[131] J. C. Strikwerda. Finite difference schemes and partial differential equations. SIAM, 2004.

[132] W. J. Stronge. Impact mechanics. Cambridge university press, 2004.

[133] H. Suzuki. Vibration and sound radiation of a piano soundboard. The Journal of the Acoustical Society of America, 80(6):1573-1582, 1986.

[134] H. Suzuki. Model analysis of a hammer-string interaction. The Journal of the Acoustical Society of America, 82(4):1145-1151, 1987.

[135] M. Taubkin and A. Del Nery. Violeiros do Brasil. Myriam Taubkin Produções Artísticas, 2008.

[136] A. Torin and M. Newton. Collisions in drum membranes: a preliminary study on a simplified system. In International Symposium on Musical Acoustics, pages 401-406, 2014.

[137] B. Trévisan. Prédiction des mécanismes vibroacoustiques des plaques orthotropes raidies de formes quelconques: Application à la table d'harmonie de piano. $\mathrm{PhD}$ thesis, Université de Lyon; INSA de Lyon (France), 2016.

[138] F. Turckheim, T. Smit, and R. Mores. Novel impulse response measurement method for stringed instruments. In Proc., 20th Int. Cong. on Ac. Sydney, pages 2840-2845, 2010. 
[139] C. Valette and C. Cuesta. Mécanique de la corde vibrante. Hermes, 1993.

[140] M. van Walstijn and J. Bridges. Simulation of distributed contact in string instruments: a modal expansion approach. In Signal Processing Conference (EUSIPCO), 2016 24th European, pages 1023-1027. IEEE, 2016.

[141] M. van Walstijn, J. Bridges, and S. Mehes. A real-time synthesis oriented tanpura model. In Proc. Int. Conf. Digital Audio Effects (DAFx-16), 2016.

[142] M. Van Walstijn and V. Chatziioannou. Numerical simulation of tanpura string vibrations. In Proc. International Symposium on Musical Acoustics, 2014.

[143] I. Vilela. The singing guitar, yo. Estudos Avançados, 24(69):323-347, 2010.

[144] I. Vilela. Cantando a própria história: Música Caipira e Enraizamento. São Paulo: Editora da Universidade de São Paulo, 2013.

[145] E. M. von Hornbostel and C. Sachs. Systematik der musikinstrumente. ein versuch. Zeitschrift für Ethnologie, 46(H. 4/5):553-590, 1914.

[146] C. P. Vyasarayani, S. Birkett, and J. McPhee. Modeling the dynamics of a vibrating string with a finite distributed unilateral constraint: Application to the sitar. The Journal of the Acoustical Society of America, 125(6):3673-3682, 2009.

[147] A. Watzky. Sur la vibration non linéaire des fils précontraints(cordes, verges tendues). $\mathrm{PhD}$ thesis, Université Pierre et Marie Curie-Paris VI, 1992.

[148] G. Weinreich. Coupled piano strings. The Journal of the Acoustical Society of America, 62(6):1474-1484, 1977.

[149] J. Woodhouse. On the synthesis of guitar plucks. Acta Acustica united with Acustica, 90(5):928944, 2004.

[150] J. Woodhouse. Plucked guitar transients: Comparison of measurements and synthesis. Acta Acustica united with Acustica, 90(5):945-965, 2004.

[151] J. Woodhouse. On the bridge hill of the violin. Acta Acustica united with Acustica, 91(1):155-165, 2005.

[152] J. Woodhouse and R. Langley. Interpreting the input admittance of violins and guitars. Acta Acustica united with Acustica, 98(4):611-628, 2012.

[153] H. Wright. The acoustics and psychoacoustics of the guitar. $\mathrm{PhD}$ thesis, $\mathrm{Ph}$. D. thesis, University of Wales, Cardiff, 1996.

[154] A. Zhang and J. Woodhouse. Reliability of the input admittance of bowed-string instruments measured by the hammer method. The Journal of the Acoustical Society of America, 136(6):33713381, 2014. 


\title{
THÈSE DE DoctoraT
}

\section{Guilherme ORELLI PAIVA}

\section{Vibroacoustic characterization and physical modelling of the viola caipira}

\author{
Caractérisation vibroacoustique et modélisation physique de la viola caipira
}

\begin{abstract}
Résumé
La viola caipira est un type de guitare brésilienne, largement utilisée dans la musique populaire. Elle comprend dix cordes métalliques organisées en cinq paires, accordées à l'unisson ou à l'octave. Le travail de thèse porte sur l'analyse des spécificités des sons musicaux produits par cet instrument, peu étudié dans la littérature.

L'analyse des mouvements des cordes pincées au moyen d'une caméra rapide montre l'importance des vibrations par sympathie qui donnent lieu à un halo sonore, constituant une signature perceptive importante. Ces mesures révèlent également l'existence de chocs entre cordes, qui ont des conséquences très clairement audibles. L'analyse des mouvements vibratoires de la caisse, menée par des moyens classiques (marteau et vibromètre laser à balayage) révèle une structure modale différente de la guitare classique. Les mobilités vibratoires au chevalet sont par ailleurs mesurées au moyen de la méthode du fil brisé, simple de mise en oeuvre et peu couteuse dans la mesure où elle évite l'utilisation d'un capteur d'effort. Associée à une analyse modale haute résolution (méthode ESPRIT), ces mesures permettent de déterminer les déformées modales aux points de couplage corde/caisse et donc de caractériser l'instrument.

Une modélisation physique, basées une approche modale, est réalisée à des fins de synthèse sonore. Elle prend en compte les mouvements des cordes selon 2 polarisations, les couplages avec la caisse ainsi que les collisions entre cordes. Ce modèle est qualifié de modèle hybride car il combine une approche analytique pour décrire les vibrations des cordes et des données expérimentales décrivant la caisse. Les simulations dans le domaine temporel rendent compte des principales caractéristiques identifiées de la viola caipira.
\end{abstract}

\section{Mots clés}

Viola caipira, instruments à cordes, acoustique musicale, modélisation physique, synthèse sonore, collisions entre cordes, vibration par sympathie

\begin{abstract}
The viola caipira is a type of Brazilian guitar widely used in popular music. It consists of ten metallic strings arranged in five pairs, tuned in unison or octave. The thesis work focuses on the analysis of the specificities of musical sounds produced by this instrument, which has been little studied in the literature.

The analysis of the motions of plucked strings using a high speed camera shows the importance of sympathetic vibrations, which results in a sound halo, constituting an important perceptive feature. These measurements also reveal the existence of shocks between strings, which lead to very clearly audible consequences. The modal analysis of the body vibrations, carried out by a scanning laser vibrometer and an automatic impact hammer reveals some differences and similarities with the classical guitar. Bridges mobilities are also measured using the wire-breaking method, which is simple to use and inexpensive since it does not require the use of a force sensor. Combined with a high-resolution modal analysis (ESPRIT method), these measurements enable to determine the modal shapes at the string/body coupling points and thus to characterize the instrument.

A physical modelling, based on a modal approach, is carried out for sound synthesis purposes. It takes into account the strings motions according to 2 polarizations, the couplings with the body and the collisions between strings. This model is called a hybrid model because it combines an analytical approach to describe the vibrations of strings and experimental data describing the body. Simulations in the time domain reveal the main characteristics of the viola caipira.
\end{abstract}

\section{Key Words}

Viola caipira, string instruments, musical acoustics, physical modelling, sound synthesis, string/string collisions, sympathetic vibration 